Metal thin film growth on multimetallic surfaces: From quaternary metallic glass to

\title{
binary crystal
}

\author{
by
}

\section{Dapeng Jing}

A dissertation submitted to the graduate faculty

In partial fulfillment of the requirements for the degree of

DOCTOR OF PHILOSOPHY

Major: Physical Chemistry

Program of Study Committee:

Patricia A. Thiel, Major Professor

James W. Evans

Mark S. Gordon

Klaus Schmidt-Rohr

Sriram Sundararajan

Iowa State University

Ames, Iowa

2010

Copyright $($ C Dapeng Jing, 2010. All rights reserved. 
Dedicated to my parents, and to my love, Peng $\mathrm{Xu}$ 


\section{TABLE OF CONTENTS}

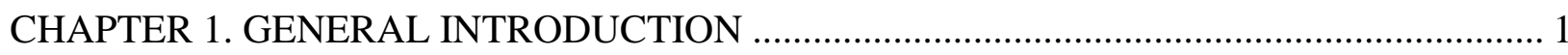

1. Thin film growth on surfaces: General concepts ............................................................. 1

2. General review: Metal thin film growth on multimetallic surfaces - glasses and

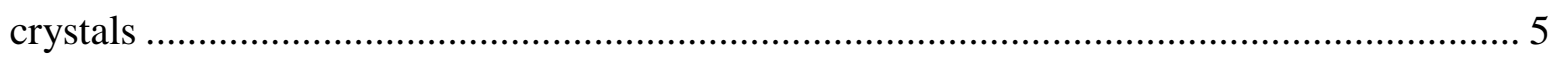

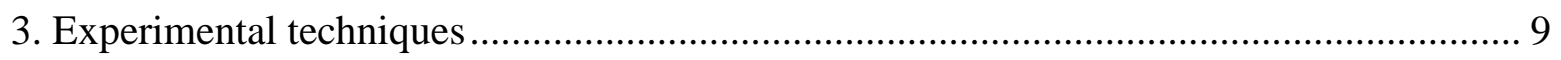

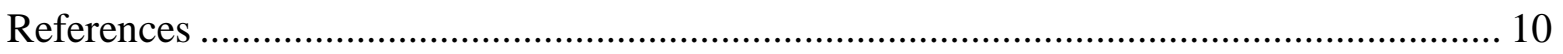

\section{CHAPTER 2. STRANSKI-KRASTONOV-LIKE GROWTH OF AN Ag FILM ON A}

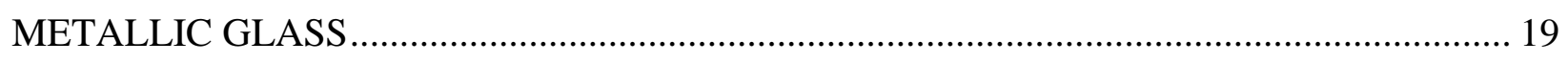

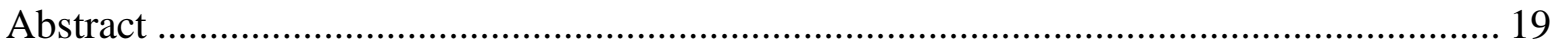

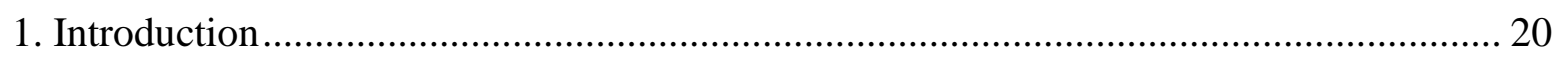

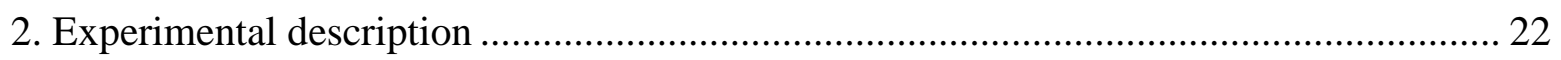

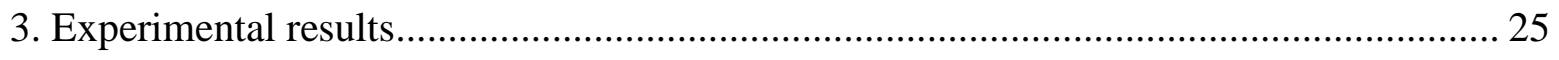

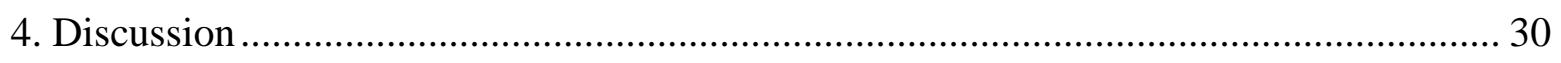

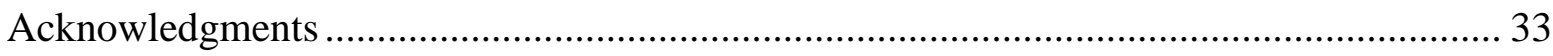

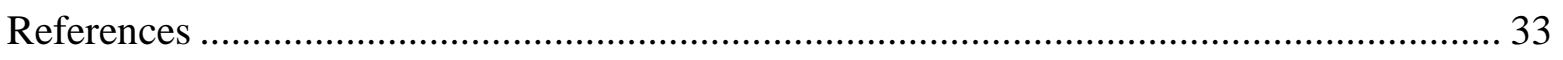

CHAPTER 3. COARSENING AND DECAY OF BILAYER Ag(110) ISLANDS ON

NiAl(110): CROSSOVER FROM SMOLUCHOWSKI RIPENING TO OSTWALD

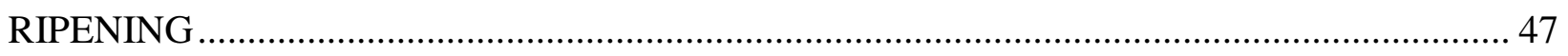

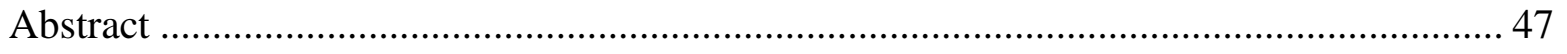

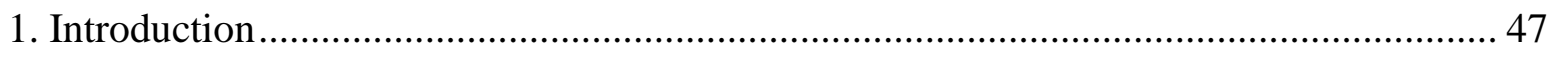

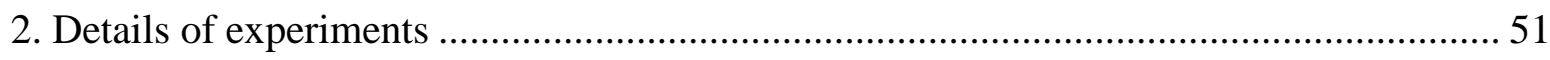

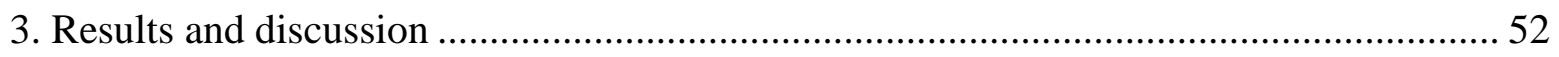




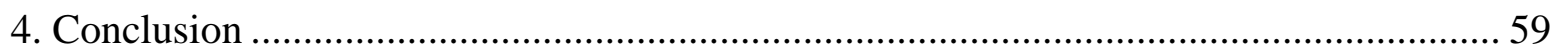

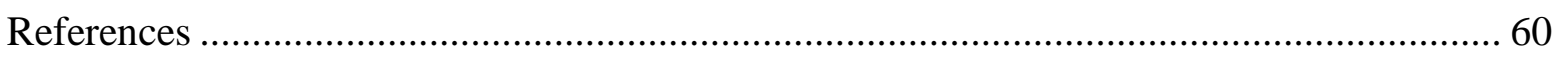

CHAPTER 4. LOW-ENERGY ELECTRON DIFFRACTION STUDY OF Ag THIN FILM

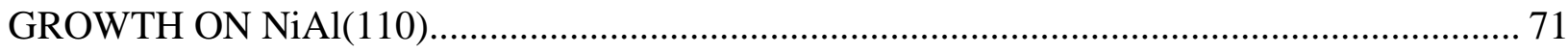

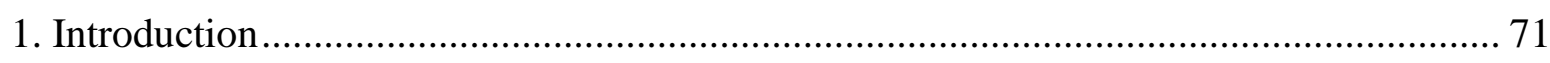

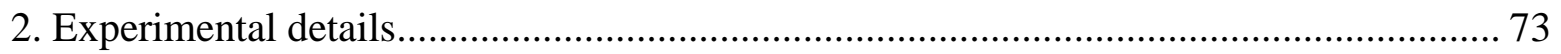

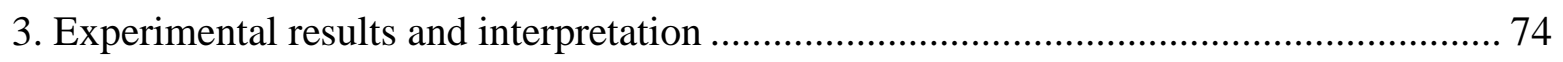

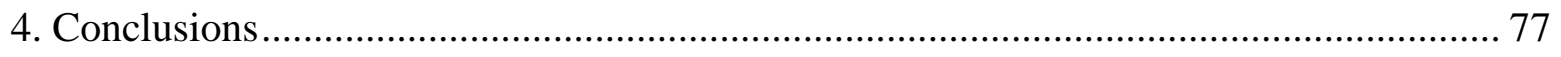

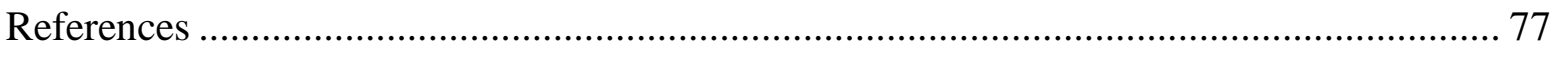

Appendix: Supplemental experimental details for chapter 4 .......................................... 106

CHAPTER 5. EPITAXIAL GROWTH OF Ni ON NiAl(110) SURFACE …………............... 114

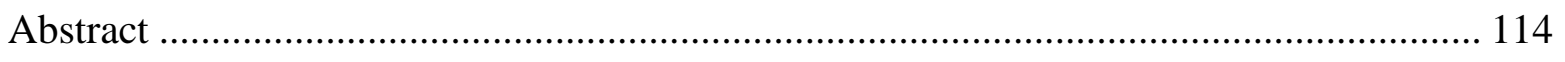

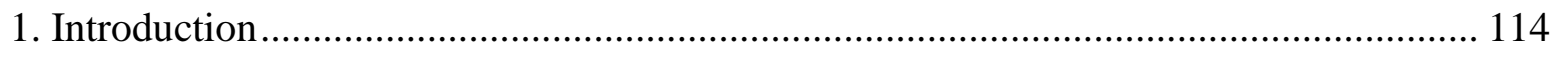

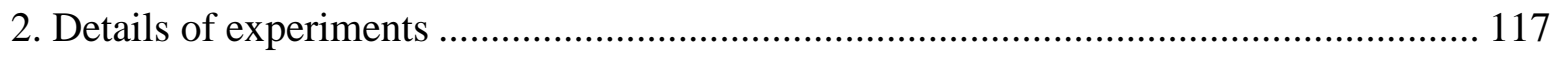

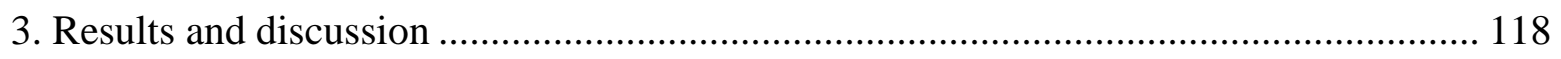

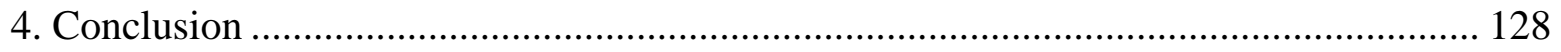

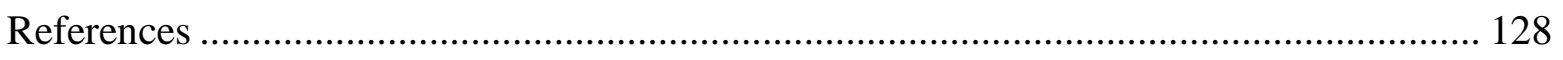

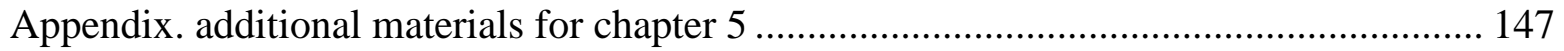

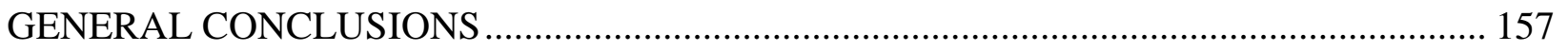

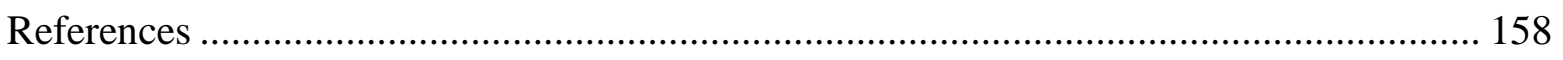

APPENDIX I. FAR-FROM-EQUILIBRIUM FILM GROWTH ON ALLOY SURFACES:

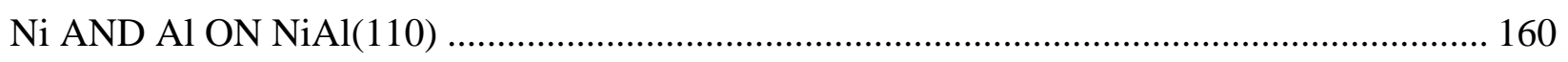

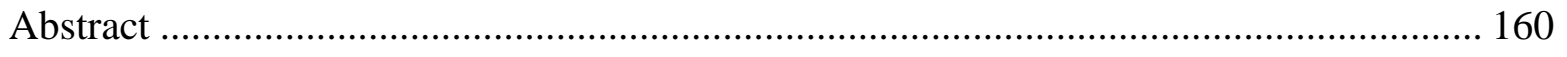

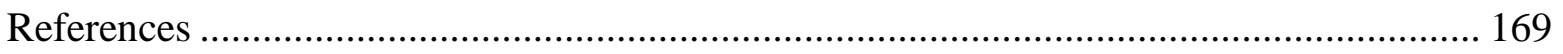


APPENDIX II. FORMATION AND COARSENING OF Ag(110) BILAYER ISLANDS ON NiAl(110): STM ANALYSIS AND ATOMISTIC LATTICE-GAS MODELING .................. 175

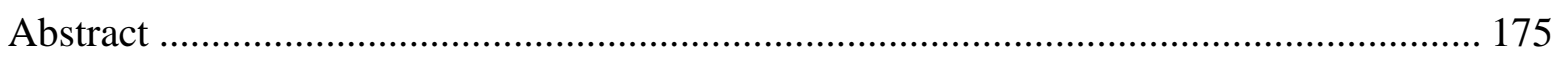

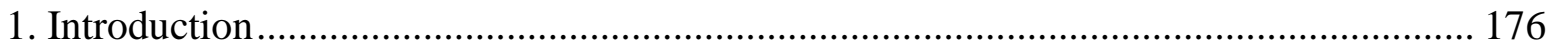

2. Experimental details, computational methods, and island structure .......................... 178

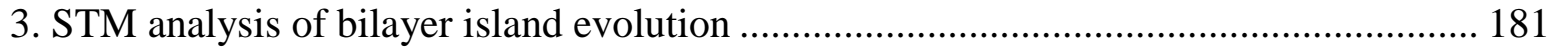

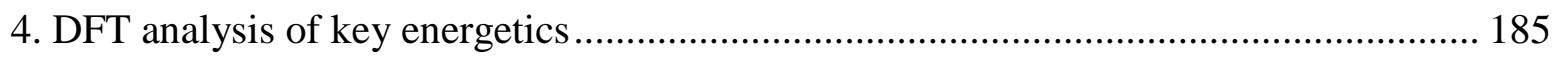

5. Atomistic lattice-gas model and KMC simulation............................................... 190

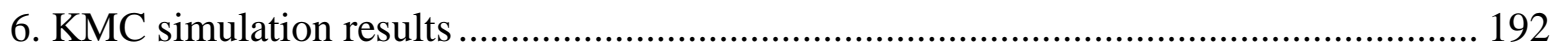

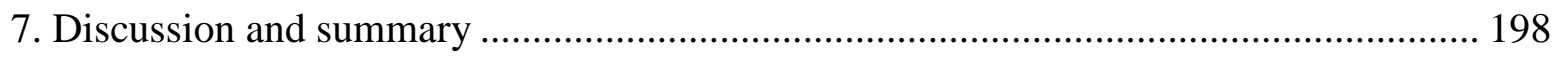

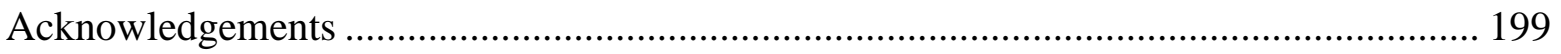

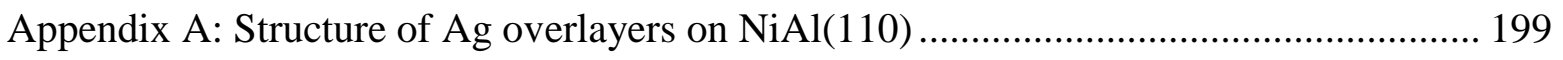

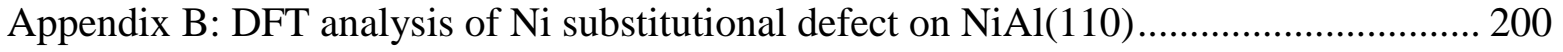

Appendix C: Rate equation analysis of defect-enhanced nucleation ............................ 201

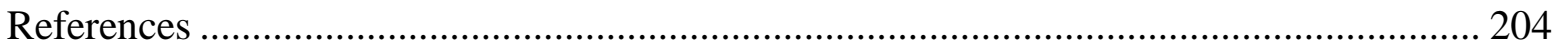

APPENDIX III. EXPERIMENTAL DATABASE ......................................................... 228

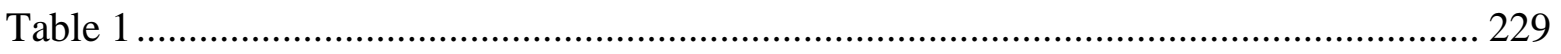

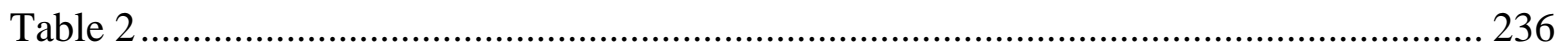

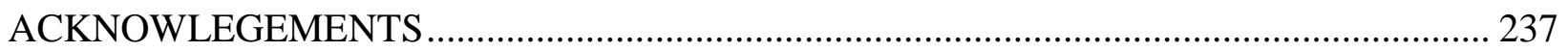




\section{CHAPTER 1. GENERAL INTRODUCTION}

\section{Thin film growth on surfaces: General concepts}

Thin films are thin material layers ranging from a fraction of a nanometer to several micrometers in thickness. Some major areas of thin film applications are microelectronics [15], optical [6] and magnetic devices [7], electrochemistry [8-10], protective [11,12] and decorative coatings [13-15] and catalysis [16-19].

There are many techniques used for the growth of thin films. A few examples are listed here. Chemical vapor deposition (CVD) is a technique in which substances react in the gas phase and at the surface of the substrate and form a solid layer on the heated substrate $[20,21]$. CVD is usually performed in a vacuum system to generate high-purity films. The chemical composition of the deposited film can be tuned by changing the gas phase composition. Therefore, films of different compositions can be generated if desired. CVD is not only used to deposit thin films, it can also be used to grow bulk materials, such as single crystals. Molecular beam epitaxy (MBE) is a technique where high-quality crystalline films can be grown on the substrate usually under ultra-high vacuum (UHV) conditions [22-24]. In a typical MBE process, pure elements are heated in separate cells until they start to sublimate, and the gas phase molecular beams are directed on the substrate followed by condensation and reaction. In $\mathrm{MBE}$, one can easily change the film composition by opening or closing one or more shutters to the cells to achieve desired properties. Physical vapor deposition (PVD) is a general term used in the thin film industry to describe a series of methods to deposit thin films. In contrast to CVD, the deposition method involves only physical processes such as evaporation and plasma sputter bombardment. In the work 
presented in this thesis, high temperature vacuum evaporation PVD is used to grow metal thin films. A thermal evaporator and electron beam evaporators are used. In a thermal evaporator, the material to be evaporated is held in a Knudsen-like cell, i.e. a container with a small orifice in which the solid comes to quasi-equilibrium with its vapor. DC current is passed through an electric resistance heater (W filament) to melt the target material and raise its vapor pressure to a useful range. So the particles of the material escape the cell and arrive at the substrate and form a solid layer. In an electron beam evaporator, a rod of the target material is heated by a beam of accelerated electrons from an electron gun. If the target material tends to melt before the vapor pressure reaches a useful value, it is usually kept in a crucible. However, the crucible has to be conductive and at the same time not reactive with the target at high temperatures.

In thin film research, the oriented growth of a crystalline film on a single-crystal substrate is referred to as epitaxy. The case where both film and substrate are of the same material is called homoepitaxy (A on A), whereas where film and substrate are different materials is called heteroepitaxy (A on B). Traditionally, epitaxial film growth has been classified into three growth modes [27]: (a) Frank-van der Merwe (FM) mode, i.e., layer-bylayer growth. In this growth mode, the film atoms are more strongly bound to the substrate than to each other. In terms of thermodynamic properties, the surface energy of the substrate $\gamma_{\mathrm{s}}$, is no less than the sum of the surface energy of the film $\gamma_{\mathrm{f}}$ and the surface energy of the substrate-film interface $\gamma_{\mathrm{s} / \mathrm{f}}$ :

$$
\gamma_{\mathrm{s}} \geq \gamma_{\mathrm{f}}+\gamma_{\mathrm{s} / \mathrm{f}}
$$

(b) Voller-Weber (VW) mode, i.e., 3D island growth. In this growth mode, the film atoms are more strongly bound to each other than to the substrate. Or one can write: 


$$
\gamma_{\mathrm{s}}<\gamma_{\mathrm{f}}+\gamma_{\mathrm{s} / \mathrm{f}}
$$

(c) Stranski-Krastanov (SK) mode, i.e., layer-plus-island growth. This mode represents the intermediate case between FM and VW growth. After an initial layer-by-layer growth, the growth of 3D islands takes place.

$$
\begin{array}{ll}
\gamma_{\mathrm{s}} \geq \gamma_{\mathrm{f}}+\gamma_{\mathrm{s} / \mathrm{f}} & \text { at } \theta \leq \theta_{\mathrm{c}} \\
\gamma_{\mathrm{s}}<\gamma_{\mathrm{f}}+\gamma_{\mathrm{s} / \mathrm{f}} & \text { at } \theta>\theta_{\mathrm{c}}
\end{array}
$$

Here $\theta$ is the coverage.

Growth modes quite different from those above are sometimes observed, e.g., height selection due to quantum size effects (QSE) [28-30]. In these growth systems, the electrons of the film material are confined within the film. For instance, in metal-on-semiconductor systems, electrons are strongly confined in the metal overlayer or nanostructure due to the large electrical resistance of the substrate [31,32]. In metal-on-metal heteroepitaxy systems, the electrons in the overlayer can also be confined if the substrate presents a relative band gap in the direction perpendicular to the surface near the Fermi level. Therefore, the film electrons are reflected at the film-substrate interface [33,34]. Confinement can even be produced by a relative "symmetry gap". This occurs, for example, if the film valence electrons close to the Fermi level have $s p$ character and the substrate $s p$ partial density of states has a gap at the Fermi level [34]. This spatial confinement of electrons along the z-axis by a $1 \mathrm{D}$ potential well results in discrete energy states (particle in a $1 \mathrm{D}$ box model) producing oscillations of total electronic energy with the thickness of the film. If the contribution of the electronic energy to the total energy is significant, then some selective film thicknesses will be energetically favored. 
Thin film growth typically occurs initially via island formation on the substrate, and subsequently by development of thicker continuous films [35-38] (See Fig. 1). Basically there are seven elementary processes involved in the formation and growth of islands, they are listed as follows: (1) Random deposition of the film material on the substrate at a certain rate $\mathrm{F}$ (In this thesis, I will use the unit of atoms per adsorption site per unit time). (2) The adatoms diffuse across the terrace between neighboring adsorption sites [35-38]. (3) Multiple diffusing atoms aggregate and nucleate to form an island on terraces or at trap sites. A critical size, $i$, is often introduced such that islands with $i+1$ atoms are considered stable. Islands with i or less atoms can dissociate and are regarded as non-stable [36,38,39]. (4) Islands grow in size due to aggregation of diffusing atoms [35-38]. (5) Upward diffusive transport (hop-up) of atoms from lower to higher layers. (6) Downward diffusive transport (hop-down) of atoms deposited in higher layers to lower layers. (7) Re-shaping of islands via movement of atoms around island edges. This process is often inhibited by the presence of an Ehrlich-Schwoeble (ES) barrier [36-38].

The atomistic processes can be described quantitatively by mean-field (MF) rate equations [39-41]. This theory was developed in the 1960s and has been very useful in elucidating the basic behavior of the mean island density for the case of isotropic terrace diffusion. The analysis of experimental island density $\mathrm{N}_{\mathrm{isl}}$ as a function of deposition rate $\mathrm{F}$ and substrate temperature $\mathrm{T}$ is useful in evaluating the island formation critical size $\mathrm{i}$, the diffusion barrier $\mathrm{E}_{\mathrm{d}}$ and the bond energy for neighboring adatoms $\mathrm{E}_{\mathrm{b}}$ [38].

Island shape is a measurable quantity that is controlled by (some of) the above parameters. It is most affected by the details of edge or periphery diffusion. If edge diffusion is slow (low surface temperature), typically ramified islands form. These islands sometimes 
have dendritic shape, or at least the island edges are rough. If the edge diffusion is fast enough (high surface temperature), the adatoms can easily cross the kink sites along island edges, and the island corners. As a result, compact islands form. In other words, the shape of a growing island might differ from the equilibrium shape due to kinetic limitations [38]. Island size distribution is another measurable quantity that can be related to (some of) the above parameters. It is affected by critical size i, coverage, substrate structure and temperature [38]. At relatively high coverages, it is also strongly influenced by coarsening phenomena. There are two mechanisms of coarsening. (1) Ostwald ripening (OR) where large islands grow at the expense of smaller islands due to the thermodynamic driving force to reduce total free energy associated with island edges [42]. (2) Coalescence where islands diffuse and merge upon contact. This process is also called Smoluchowski ripening (SR) [43].

\section{General review: Metal thin film growth on multimetallic surfaces - glasses and crystals}

A metallic glass is the subject of a part of this thesis. A metallic glass is an intermetallic material with a disordered atomic-scale structure. In contrast to most metals, which are crystalline, metallic glasses are amorphous alloys. Such a disordered structure is produced directly from the liquid state during fast cooling. If the shortest dimension of a metallic glass exceeds $1 \mathrm{~mm}$, it is called a bulk metallic glass [44]. Metallic glasses have many technological useful properties, such as high strength/weight ratio, high wear resistance and high corrosion resistance [45]. 
While most studies of bulk metallic glasses (BMGs) are focusing on the growth development and improvement of mechanical properties, far less attention has been paid to their fundamental surface properties. From literature, one can draw the following pertinent generalizations. First, atomic force microscopy (AFM) [46.47] and scanning tunneling microscopy (STM) [48] can be used to study the topology and morphology of the BMG surface. The surface morphology usually does not depend on the preparation or cleaning process. It rather reflects the intrinsic surface structure of the glasses [48]. The surface of a BMG is typically very flat. The surface features are on the order of several nanometers (height) over a $10 \times 10 \mu \mathrm{m}^{2}$ region. There is no long-range order on the surface. However, local periodic corrugations are sometimes observed due to relief of nonhydrostatic thermal stress [46]. Second, X-ray photoelectron spectroscopy (XPS) [49] and Auger electron spectroscopy (AES) $[47,49-51]$ can be used to characterize the surface chemical composition. In general, the surface composition is different from the bulk due to two reasons: (1) Metallic glasses are thermodynamically metastable, hence their reactivity is essentially higher than that of their crystalline counterpart. The surface is easily oxidized to form an oxide layer and hence reduce the surface energy. The preferential oxidation of the most reactive constituent leads to an increase of its surface concentration [49]. (2) Even in the absence of oxidation, surface segregation occurs and leads to the enrichment of certain constituents, often those that have the least surface energy and/or largest atomic radius $[49,50]$.

There are also metal thin film growth studies on BMG surfaces both experimentally and theoretically. Imai et. al. have grown Ag thin films using pulsed laser deposition (PLD) on a $\mathrm{Cu}-\mathrm{Zr}-\mathrm{Ti} \mathrm{BMG}$ surface [51]. They found that Ag forms a wetting layer and covers the entire metallic glass surface. The film effectively prevents the metallic glass surface from 
being oxidized. It also improves the wettability of the surface for $\mathrm{Sn}-\mathrm{Ag}-\mathrm{Cu}$ solder. Papageorgiou et. al. have used molecular dynamics (MD) simulations to study $\mathrm{Zr}$ and $\mathrm{Cu}$ thin film deposition on $\mathrm{Cu}_{46} \mathrm{Zr}_{54}$ bulk metallic glass [52]. They found atomic rearrangement takes place at the film-substrate interface at the very early stage of the film growth process. $\mathrm{Zr}$ tends to form a crystalline adlayer whereas $\mathrm{Cu}$ forms a partially crystallized adlayer.

Turning now to the crystalline alloy which is the subject of a part of this thesis, transition metal (TM) aluminides are a series of intermetallics that have $\mathrm{Al}$ as a constituent. Some of these intermetallics have very interesting properties such as high heat-resistance, high corrosion and oxidation resistance and good strength to weight ratio [53]. Among the valuable TM aluminides, bcc-NiAl has been extensively studied both experimentally and theoretically. The atomic and electronic structure of its (110) surface has also been wellcharacterized. The $\mathrm{NiAl}(110)$ surface is bulk-terminated, consisting of rows of $\mathrm{Ni}$ and $\mathrm{Al}$ atoms in a 1:1 stoichiometry. The top-most layer of $\mathrm{Al}$ atoms are $0.02 \mathrm{~nm}$ higher than their in-plane Ni neighbors, and the first interlayer spacing is a few percent higher than that of the deeper layers [54]. In terms of electronic structure, the $\mathrm{NiAl}(110)$ surface has a small depression in the density of states at the Fermi edge [55]. This pseudo band gap reduces the electronic interaction between the substrate and the adspecies, making it a good substrate for studying growth of one-dimensional atomic wires [56-58].

In contrast to the NiAl surface, research shows the low-index surfaces of another CsCl-type TM aluminide - FeAl - undergoes surface reconstructions [59-61]. In the case of the (110) surface, it has been shown that there is an attenuation of $\mathrm{Al}$ concentration due to preferential sputtering. However, upon annealing, Al segregation is promoted resulting a surface reconstructed phase. AES and low energy electron diffraction (LEED) studies 
indicate the surface reconstructs into an incommensurate structure consistent with a $\mathrm{FeAl}_{2}$ stoichiometry [61].

One should note that one of the significant features of the TM aluminides is that they can serve as templates for the growth of well-ordered oxide layers [62-66]. Due to their importance in a broad range of applications, thin oxide layers and their surfaces have attracted considerable attention. Among the oxides, alumina has been the focus of numerous studies. Due to lattice mismatch, the production of alumina thin film from oxidation of pure aluminum results in rough surfaces and short-range crystalline order. However, research has shown that the oxidation of some of the TM aluminides at high temperature can successfully produce well-ordered alumina films. Specifically, upon direct oxidation of clean alloy surfaces at high temperature, well-ordered thin alumina films have been identified on $\mathrm{Ni}_{3} \mathrm{Al}(111)$ [66], $\mathrm{NiAl}(111)$ [65], $\mathrm{NiAl}(001)$ [64], $\mathrm{NiAl}(110)$ [62,63] and $\mathrm{FeAl}(110)$ [61]. Metal thin film growth on TM aluminide studies are rare, partly due to the fact that alloy substrates pose significant complications, such as distinct types of adsorption sites and diffusion pathways. However, choosing binary metallic alloys as substrates for metal thin film growth provides significant additional possibilities for guiding film structure and morphology. Ho et. al. studied Au dimers [56] and 1D atomic chains [57] on $\mathrm{NiAl}(110)$ using STM tip manipulation of single atoms. Even though these studies do not fall into the film growth category, their results are useful in understanding various phenomena in thin film growth such as adsorption site and nucleation. McCarty et. al. have studied Al deposition on $\mathrm{NiAl}(110$ [67] in the temperature range from $600 \mathrm{~K}$ to $900 \mathrm{~K}$. They find that the composition of the growing film differs from the deposited material $-\beta-\mathrm{NiAl}$ forms instead of pure $\mathrm{Al}$. In other words, the substrate provides a net Ni flux to its surface. They also observed localized 
changes in surface topography and composition associated with motion of dislocations. They conclude that for a reactive surface, the topography and composition can evolve during heteroepitaxy via the motion of point and line defects within the substrate material. In our group, we have studied Ag thin film growth on NiAl(110) [68]. STM studies reveal an initial bilayer growth of Ag islands, in which the film appears to adopt fcc $\mathrm{Ag}(110)$-like structure, at least for the first two levels. Density functional theory (DFT) analysis shows that the bilayer growth mode is promoted by QSE.

\section{Experimental techniques}

The experiments in this thesis were performed in two ultra-high vacuum (UHV) chambers. The STM studies were performed in one UHV chamber equipped with an Omicron variable-temperature STM, AES, XPS and LEED. The base pressure of the chamber was $2 \times 10^{-11}$ Torr. The VT-STM was the principle surface analytical tool. It has several advantages in studying metal surfaces. First, STM, unlike diffraction techniques such as LEED, provides real-space images, revealing real-space microscopic surface structures and film growth characteristics. Second, since in typical measurements, the STM tip does not physically touch the sample surface during measurements, it usually preserves the surface structure. Third, STM (generally) provides better spatial resolution than its competitors, capable of resolving nanometer scale surface features. And last, STM provides sequential real-time imaging (STM movies). This enables us to study the dynamics of surface structures. An excellent summary of STM operation and applications can be found in C. J. Chen's book [69], "Introduction to Scanning Tunneling Microscopy”. 
The LEED studies were performed in another UHV chamber equipped with a Varian

LEED system and a quadrupole mass spectrometer (MS). The base pressure was $8 \times 10^{-11}$ Torr. LEED was the primary tool. It is a technique for the determination of the surface structure of a crystalline material by bombardment with a beam of low energy electrons (30$300 \mathrm{eV}$ ) and observation of diffracted electrons as bright spots on a fluorescent screen [70,71]. The information on film morphology and reconstruction can be extracted from the diffraction patterns. For film depositions, $\mathrm{Ag}$ and $\mathrm{Au}$ were evaporated from a homemade electron beam evaporator (See Fig. 2).

\section{References}

[1] S. Bosch, J. Ferre-Borrull, J. Sancho-Parramon, Solid-State Electron. 45/5 (2001) 703.

[2] U. Hasenkox, Fortschr.-Ber. VDI, Reihe 9306 (1999) I.

[3] S. Hoffmann, R. Waser, Fortschrittsber. Dtsch. Keram. Ges. 10/4, Keramische Schichten (1995) 203.

[4] M.S. Silverstein, R. Chen, E. Sacher, L. Sandrin, Met. Polym. 2, [Pap. Montreal Workshop], 2nd (2002) 61.

[5] C. Yu, S. Kim, S. Meikle, T.T. Doan, G. Blalock, Materials Research Society Symposium Proceedings 264/Electronic Packaging Materials Science VI (1992) 401.

[6] K. Lewis, Int. Trends Appl. Opt. (2002) 187.

[7] N. Smith, J Appl Phys 90/11 (2001) 5768.

[8] A. Patil, V. Patil, J.-W. Choi, H.-J. Kim, B.-H. Cho, S.-J. Yoon, J. Electroceram. 23/2-4 (2009) 230. 
[9] A. Andreas Arie, W. Chang, J. Kee Lee, J. Electroceram. 24/4 308.

[10] H.-S. Kim, H.S. Ahnn, J.H. Kim, J.H. Park, Y.-K. Sun, C.S. Yoon, Journal of the Electrochemical Society 157/6 A636.

[11] N. Ledermann, J. Baborowski, P. Muralt, N. Xantopoulos, J.M. Tellenbach, Surf. Coat. Technol. 125/1-3 (2000) 246.

[12] M. Stueber, H. Holleck, H. Leiste, K. Seemann, S. Ulrich, C. Ziebert, Journal of Alloys and Compounds 483/1-2 (2009) 321.

[13] A.C. Fernandes, F. Vaz, L. Cunha, N.M.G. Parreira, A. Cavaleiro, P. Goudeau, E. Le Bourhis, J.P. Riviere, D. Munteanu, B. Borcea, R. Cozma, Thin Solid Films 515/13 (2007) 5424.

[14] H. Hoche, S. Gross, R. Foerster, J. Schmidt, W. Adamitzki, Plasma Processes Polym. 6/S1 (2009) S671.

[15] M.T. Mathew, E. Ariza, L.A. Rocha, A.C. Fernandes, F. Vaz, Tribol. Int. 41/7 (2008) 603.

[16] G.A. Rimbu, M. Iordoc, R. Vasilescu-Mirea, I. Stamatin, T. Zaharescu, Rev. Chim. (Bucharest, Rom.) 60/12 (2009) 1285.

[17] A.T. Carvalho, A.P. Nascimento Filho, L.M. Silva, M.L.P. Silva, J.C. Madaleno, L. Pereira, Mater. Sci. Forum 514-516/Pt. 2, Advanced Materials Forum III (2006) 1328.

[18] Y. Fujisaki, K. Kushida-Abdelghafar, Y. Shimamoto, H. Miki, J Appl Phys 82/1 (1997) 341 .

[19] H. Wang, Z. Wang, L. Huang, A. Mitra, Y. Yan, Langmuir 17/9 (2001) 2572. 
[20] A.C. Jones, M.L. Hitchman, Editors, Chemical Vapour Deposition; Precursors, Processes and Applications, 2009.

[21] H.O. Pierson, Editor, Handbook of Chemical Vapor Deposition: Principles, Technology and Applications, 2nd Edition, 2000.

[22] R.F.C. Farrow, Editor, Molecular Beam Epitaxy: Applications to Key Materials, 1995.

[23] M.A. Herman, H. Sitter, Molecular Beam Epitaxy: Fundamentals and Current Status, Second Revised and Updated Edition, 1996.

[24] Z.R. Wasilewski, R. Beresford, J.A. Gupta, Editors, Molecular Beam Epitaxy, 2009.

[25] J.E. Mahan, Physical Vapor Deposition of Thin Films, 2000.

[26] D.M. Mattox, Editor, Handbook of Physical Vapor Deposition (PVD) Processing: Film Formation, Adhesion, Surface Preparation and Contamination Control, 1998.

[27] E. Bauer, Zeitschrift fuer Kristallographie 110 (1958) 372.

[28] D.A. Evans, M. Alonso, R. Cimino, K. Horn, Phys Rev Lett 70/22 (1993) 3483.

[29] M.C. Tringides, M. Jalochowski, E. Bauer, Phys. Today 60/4 (2007) 50.

[30] M. Hupalo, S. Kremmer, V. Yeh, L. Berbil-Bautista, E. Abram, M.C. Tringides, Surf Sci 493/1-3 (2001) 526.

[31] S.M. Binz, M. Hupalo, M.C. Tringides, J Appl Phys 105/9 (2009) 094307/1.

[32] M. Li, C.Z. Wang, J.W. Evans, M. Hupalo, M.C. Tringides, K.M. Ho, Phys. Rev. B: Condens. Matter 79/11 (2009) 113404/1.

[33] T.C. Chiang, Surface Science Reports 39/7-8 (2000) 181.

[34] M. Milun, P. Pervan, D.P. Woodruff, Rep Prog Phys 65/2 (2002) 99. 
[35] R. Kern, G. Le Lay, J.J. Metios, "Basic Mechanisms in the early stages of epitaxy" in Current topics in materials science, Vol. 3, edited by E. Kaldis, Amsterdam: North Holland, 1979, pp. 131-419.

[36] T. Michely, J. Krug, Islands, Mounds, and Atoms, Springer, Berlin, 2004.

[37] A. Pimpinelli, J. Villain, Physics of Crystal Growth, Cambridge: Cambridge University Press, 1998.

[38] J.W. Evans, P.A. Thiel, M.C. Bartelt, Surface Science Reports 61/1-2 (2006) 1.

[39] J.A. Venables, Philos Mag 27/3 (1973) 697.

[40] S. Stoyanov, D. Kashchiev, Curr. Top. Mater. Sci. 7 (1981) 69.

[41] J.A. Venables, G.D.T. Spiller, M. Hanbucken, Rep Prog Phys $47 / 4$ (1984) 399.

[42] W. Ostwald, Lehrbuch der allgemeinen Chemie, Leipzig, Germany, 1896.

[43] M. Smoluchowski, Physikalische Zeitschrift 17 (1916) 557 and 585.

[44] H.S. Chen, Acta Metall. 22/12 (1974) 1505.

[45] W.H. Wang, C. Dong, C.H. Shek, Materials Science \& Engineering, R: Reports R44/2-3 (2004) 45.

[46] M.A. LaMadrid, S.D. O'Connor, A. Peker, W.L. Johnson, J.D. Baldeschwieler, J Mater Res 11/6 (1996) 1494.

[47] K.S. Nakayama, Y. Yokoyama, T. Sakurai, A. Inoue, Appl Phys Lett 90/18 (2007).

[48] D.E. Burgler, C.M. Schmidt, D.M. Schaller, F. Meisinger, T.M. Schaub, A. Baratoff, H.J. Guntherodt, Phys Rev B 59/16 (1999) 10895.

[49] K. Hashimoto, ISIJ Int. 29/7 (1989) 539.

[50] P. Patsalas, A. Lekatou, E. Pavlidou, S. Foulias, M. Kamaratos, G.A. Evangelakis, A.R. Yavari, Journal of Alloys and Compounds 434-435 (2007) 229. 
[51] A. Imai, M. Katayama, S. Maruyama, H. Nishikawa, T. Wada, H. Kimura, M. Fukuhara, T. Takemoto, A. Inoue, Y. Matsumoto, J Mater Res 24/9 (2009) 2931.

[52] D.G. Papageorgiou, A. Ibenskas, C.E. Lekka, G.A. Evangelakis, Reviews on Advanced Materials Science 18/1 (2008) 98.

[53] R.L. Fleischer, D.M. Dimiduk, H.A. Lipsitt, Annu. Rev. Mater. Sci. 19 (1989) 231.

[54] H.L. Davis, J.R. Noonan, Phys Rev Lett 54/6 (1985) 566.

[55] S.C. Lui, M.H. Kang, E.J. Mele, E.W. Plummer, D.M. Zehner, Phys. Rev. B: Condens. Matter 39/18 (1989) 13149.

[56] N. Nilius, T.M. Wallis, M. Persson, W. Ho, Phys Rev Lett 90/19 (2003) 196103/1.

[57] N. Nilius, T.M. Wallis, W. Ho, Science (New York, N.Y.) 297/5588 (2002) 1853.

[58] J. Hong, Phys. Rev. B: Condens. Matter 73/9 (2006) 092413/1.

[59] H. Graupner, L. Hammer, K. Mueller, D.M. Zehner, Surf Sci 322/1-3 (1995) 103.

[60] L. Hammer, H. Graupner, V. Blum, K. Heinz, G.W. Ownby, D.M. Zehner, Surf Sci 413 (1998) 69.

[61] O. Kizilkaya, D.A. Hite, D.M. Zehner, P.T. Sprunger, Surf Sci 529/1-2 (2003) 223.

[62] J. Libuda, F. Winkelmann, M. Baeumer, H.J. Freund, T. Bertrams, H. Neddermeyer, K. Mueller, Surf Sci 318/1-2 (1994) 61.

[63] R.M. Jaeger, H. Kuhlenbeck, H.J. Freund, M. Wuttig, W. Hoffmann, R. Franchy, H. Ibach, Surf Sci 259/3 (1991) 235.

[64] P. Gassmann, R. Franchy, H. Ibach, Surf Sci 319/1-2 (1994) 95.

[65] R. Franchy, M. Wuttig, H. Ibach, Surf Sci 189-190 (1987) 438.

[66] S.G. Addepalli, B. Ekstrom, N.P. Magtoto, J.S. Lin, J.A. Kelber, Surf Sci 442/3 (1999) 385 . 
[67] J.P. Pierce, N.C. Bartelt, K.F. McCarty, Phys Rev Lett 99/2 (2007) 026101.

[68] B. Unal, F. Qin, Y. Han, D.J. Liu, D.P. Jing, A.R. Layson, C.J. Jenks, J.W. Evans, P.A. Thiel, Phys Rev B 76/19 (2007).

[69] C.J. Chen, Introduction to Scanning Tunneling Microscopy, Oxford University Press, New York, 1993.

[70] G. Ertl, J. Kuppers, Low Energy Electrons and Surface Chemistry, VCH, Weinheim, 1974.

[71] S.-L. Chang, P.A. Thiel, in: D.A. Glocker, S.I. Shah (Eds.), Handbook of Thin Film Process Technology, Institute of Physics Publishing, Bristol and Philadelphia, 1995. 


\section{Figure captions}

Fig. 1. Schematic diagram illustrating the elementary atomic processes involved in the formation and growth of islands.

Fig. 2. Schematic diagram of the homemade electron beam evaporator. (a) Sample. (b)

Shutter. (c) W filament. (d) Crucible. (e) 5 V DC power supply. (f) $200 \Omega$ resistor. (g)

Voltmeter. (h) $5 \mathrm{kV}$ DC power supply. (i) Picoammeter. Emission current is measured by voltage across the resistor. Ion flux current is measured by the picoammeter. 
Figures

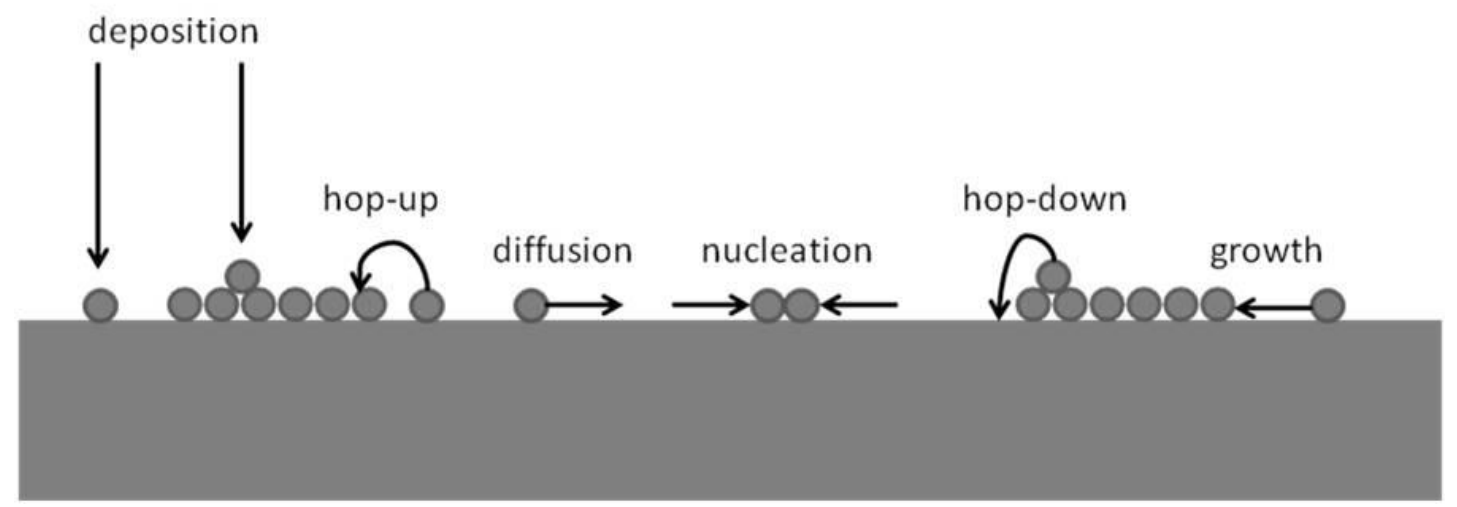

Fig. 1 


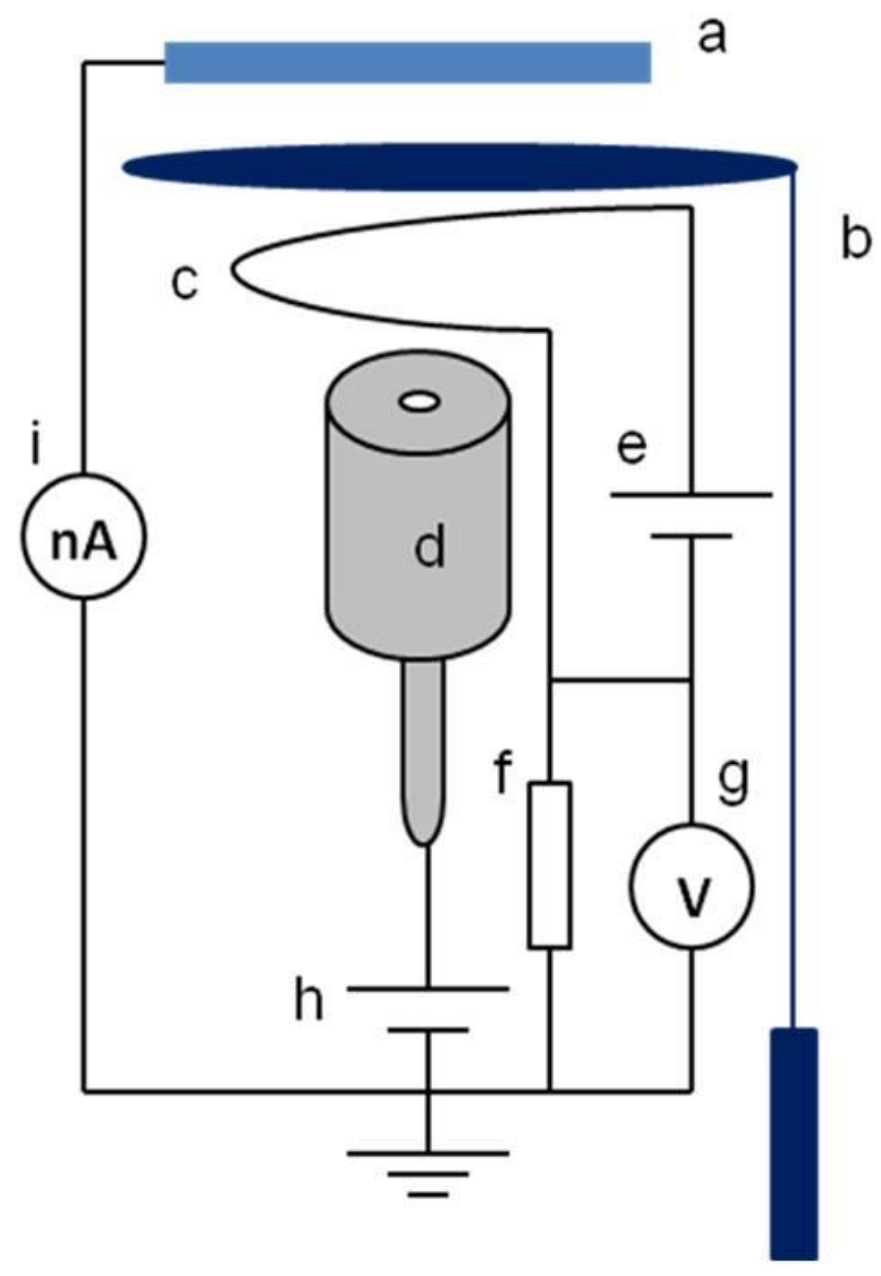

Fig. 2 


\title{
CHAPTER 2. STRANSKI-KRASTONOV-LIKE GROWTH OF AN Ag FILM ON A METALLIC GLASS
}

\author{
A paper published in Thin Solid Films \\ Dapeng Jing, Barış Ünal, Feili Qin, Chad Yuen, J.W. Evans, C.J. Jenks, D.J. Sordelet, and \\ P.A. Thiel

\begin{abstract}
We have studied the morphology of a bulk-crystalline metal, Ag, deposited on a clean surface of a metallic glass, Zr-Ni-Cu-Al. At 190-300 K, the films exhibit StranskiKrastanov-like growth, wherein three-dimensional clusters form atop a Ag wetting layer that is 4-5 monolayers thick. Above this coverage, cluster growth competes with growth of the flatter regions. The cluster density increases with decreasing temperature, indicating that the conditions of island nucleation are far-from-equilibrium. Within a simple model where clusters nucleate whenever two mobile Ag adatoms meet, the temperature-dependence of cluster density yields a (reasonable) upper limit for the value of the Ag diffusion barrier on top of the Ag wetting layer of $0.32 \mathrm{eV}$. Overall, this prototypical study suggests that it is possible to grow films of a bulk-crystalline metal that adopt the amorphous character of a glassy metal substrate, if film thickness is sufficiently low.
\end{abstract}




\section{Introduction}

The growth of thin films on metallic glasses is intriguing from both scientific and technological points of view. From a purely fundamental perspective, one might expect that growth on amorphous substrates is more difficult to analyze than on perfect single crystal substrates. However, behavior for the latter is often quite sensitive to the system-specific details (diffusion barriers across terraces, around island edges, and between layers) and film morphology reflects crystallographic anisotropies. In contrast, for amorphous substrates, behavior might be analyzed in terms of more generic models describing self-affine growth and isotropic film morphologies [1].

A particularly relevant example of the latter scenario is a study by Reinker et al. [2] of the growth by co-deposition of an amorphous ternary $\mathrm{Zr}$-Al-Cr film on oxidized Si. Indeed, initial growth up to $\sim 30 \mathrm{~nm}$ does have self-affine characteristics with isotropic morphology plausibly described by a Wolf-Villain model [1, 2]. However, in this system, there is a breakdown of the self-affine model for thicker films where features of a characteristic length develop. More generally, growth of thick films on amorphous substrates is often characterized by structure-zone models which focus on film texture and the morphology of the crystalline grains of the film material which eventually develop [3].

Another interesting feature of the growth of amorphous or glassy materials by thin film deposition is the fact that the incommensurate atomic interface between substrate and amorphous film structures can establish non-equilibrium stresses that drive the system far from equilibrium. This behavior can be used to study contrasting glass stability and crystallization dynamics compared to bulk glasses having the same composition. For example, studies with Ni-Y films deposited onto alumina substrates exhibited a much wider 
range of glass-forming compositions compared to synthesis via traditional rapid liquidquenching routes [4].

From a technological perspective, deposition of thin films on the surfaces of metallic glasses may lead to the ability to tailor surface and near-surface properties to achieve desired functionalities. As an example, metallic glasses have been considered as hydrogen-permeable membranes for applications such as high-purity hydrogen production for the fabrication of semiconductors and operation of fuel cells [5]. Metallic glasses offer the potential advantage of increased resistance to hydrogen embrittlement compared to crystalline materials, which suffer from cyclic phase transformations as hydrogen enters and leaves the lattice; moreover, the high elastic limit and isotropic structure of metallic glasses has been suggested to further resist structural degradation due to embrittlement [6-8]. To date most metallic membrane materials are based on Pd because of its pronounced hydrogen selectivity, but the high cost of Pd has motivated many studies to design alternative alloys or significantly reduce the content of Pd without diminishing the effectiveness of the alloy [9]. One tactic that can be employed in the use of other materials which oxidize more readily, such as Zr-based alloys, is to coat them with a hydrogen-permeable precious metal [10].

In this paper, our goal is to obtain fundamental insight into the nucleation and growth of metal films, particularly precious metals, at low coverages on metallic glass surfaces. Specifically, we report a study of a noble metal, $\mathrm{Ag}$, deposited on a $\mathrm{Zr}-\mathrm{Cu}-\mathrm{Ni}$-Al glass. The Ag is deposited via physical vapor deposition at room temperature and below, in ultrahigh vacuum (UHV). Our goal is to understand how the glassy nature of the substrate affects the structure and morphology of the Ag film up to about 20 monolayers (ML). To that end, we investigate the film using, primarily, scanning tunneling microscopy (STM). 


\section{Experimental description}

\section{1. $\mathrm{Zr}-\mathrm{Cu}-\mathrm{Ni}-\mathrm{Al}$ samples}

An amorphous $\mathrm{Zr}_{65} \mathrm{Cu}_{15} \mathrm{Ni}_{10} \mathrm{Al}_{10}$ specimen was synthesized by the Materials Preparation Center at the Ames Laboratory [11]. Specifically, high-purity elemental constituents (>99.99\%) were arc melted together and then drop cast to form a $6 \mathrm{~mm}$ diameter rod. A portion of the cast rod was cut and inductively remelted in a graphite crucible, where the liquid was superheated to around $1200 \mathrm{~K}$ and then injected via an over-pressure of Ar into a water-cooled $3 \mathrm{~mm}$ diameter x $40 \mathrm{~mm}$ long $\mathrm{Cu}$ mold. Graphite crucibles were used to eliminate the possibility of oxygen contamination that can occur with fused silica crucibles; the bulk oxygen content of the as-cast rod was $\sim 180$ ppmw.

The structure of the as-cast $3 \mathrm{~mm}$ diameter $\mathrm{Zr}_{65} \mathrm{Cu}_{15} \mathrm{Ni}_{10} \mathrm{Al}_{10}$ specimen was studied by X-ray diffraction $\left(\mathrm{Cu}-\mathrm{K}_{\alpha}\right)$ and differential scanning calorimetry. No indication of crystalline phases was observed in the diffraction data. Thermal analysis further confirmed that the cast rod was amorphous. Glass transition and crystallization temperatures of 650 and $753 \mathrm{~K}$,

respectively, were measured at a heating rate of $40 \mathrm{~K} \mathrm{~min}^{-1}$, which are in very close agreement with Kawamura's measurements on melt spun and injection cast materials with the same composition $[12,13]$.

\subsection{Surface studies}

Two separate samples were used, sequentially, in these studies. On each sample, two flat surfaces were polished using standard metallographic techniques, down to $0.25 \mu \mathrm{m}$ diamond paste. The final samples were rectangular, with dimensions $9 \mathrm{~mm}$ x $3 \mathrm{~mm}$ x $1 \mathrm{~mm}$. 
Because data from the two were similar, data from both samples are used without distinction in this presentation unless noted otherwise. Each polished sample was mounted on an Omicron heater and introduced into an UHV chamber equipped with Auger electron spectroscopy (AES), Low Energy Electron Diffraction, and variable-temperature STM. The base pressure of the chamber was $3 \times 10^{-9} \mathrm{~Pa}$. The sample was cleaned by $\mathrm{Ar}^{+}$sputtering (1.5 $\mathrm{keV}$ ion energy, incident beam perpendicular to the surface plane, $\mathrm{T}=300 \mathrm{~K}$ ) until the surface was judged clean by AES. It was not annealed after sputtering, to avoid crystallization. A depth profile is shown in Fig. 1, starting from a sample introduced from air. AES line intensities were converted into atomic percentages using published sensitivity factors [14].

In the film growth studies, Ag was evaporated from a commercial Omicron source, modified with a pinhole cap to make it more like a Knudsen cell. The base pressure was below $10^{-8} \mathrm{~Pa}$ during deposition. The Ag flux was $6.7 \times 10^{-3} \mathrm{ML} / \mathrm{s}$. This value was derived from STM-based coverage measurements of another system with established characteristics (Ag on $\mathrm{NiAl}(110)$ [15]). Sample surface temperature measurements were taken by means of a silicon diode at the STM coupling stage, using a calibration provided by the manufacturer (Omicron). The temperature accuracy was $\pm 5 \mathrm{~K}$ according to the manufacturer.

\subsection{Check for Ag alloying}

The major constituent of the alloy is $\mathrm{Zr}$, and the bulk phase diagram of $\mathrm{Zr}$-Al shows negligible solubility [16]. However, surface alloys can form that are not predicted based on bulk thermodynamics. Using surface energies, Christensen et al. have predicted a very small driving force for surface alloying between $\mathrm{Ag}$ films and bulk $\mathrm{Zr}$ substrates. Furthermore, it is 
possible that the other constituents in the alloy could promote alloying. Therefore, we designed a different type of experiment to check for alloying of Ag with a very similar metallic glass, $\mathrm{Zr}_{57} \mathrm{Cu}_{15.4} \mathrm{Ni}_{12.6} \mathrm{Al}_{10} \mathrm{Nb}_{5}$.

We prepared a mixture of powdered Ag and powdered metallic glass, and extruded it at $693 \mathrm{~K}$. This formed a sample with separate regions of metallic glass and Ag, as shown by the scanning electron micrograph in Fig. 2. The extruded rod was then cut, polished, and etched with nitric acid, which selectively removed the bulk Ag. If alloying had occurred at the extrusion temperature, we expected $\mathrm{Ag}$ to remain as a component of the glass after the etch.

Scanning Auger microscopy was used to search for Ag. After light ion bombardment to remove atmospheric contaminants, no Ag was detectable in the metallic glass regions, not even near the edges where a surface alloy would form. A few localized regions of high Ag concentration were found, but these could be reasonably attributed to small regions of bulk $\mathrm{Ag}$ which were not accessible to the acid. Given that the limit of detection in Auger electron spectroscopy is better than 1 at \%, we conclude that Ag does not form an alloy with the metallic glass, at least up to $693 \mathrm{~K}$.

Furthermore, the crystallization temperature of the glass, measured by differential scanning calorimetry $(778 \mathrm{~K})$, was constant within $3 \mathrm{~K}$ for samples that had been prepared with Ag and then etched, or without Ag entirely. This supports the conclusion that Ag did not alloy with the glass during extrusion. 


\section{Experimental results}

\subsection{Clean surface}

In order to interpret the structure of the films that we deposit, it is appropriate first to characterize the surface morphology of the metallic glass, which is prepared by sputtering (cf. Sec. 2). Its structure is shown in Fig. 3 at different magnifications. At high magnification, small depressions are visible that are $0.5-0.7 \mathrm{~nm}$ deep and about 4-6 $\mathrm{nm}$ wide. Two examples are indicated by the arrows in Fig. 3a. With decreasing magnification, a different type of depression becomes visible, both deeper (2-3 nm) and wider (about 70-150 nm). One such depression is close to the center of Fig. 3c. Hence, surface depressions exist on two different length scales within the range of length scales that was probed.

The root-mean-square (rms) roughness, $\sigma_{\mathrm{L}}$, is given in Fig. 4 as a function of linear dimension (L) of the region being imaged. At small L (high magnification), the roughness of the glass is about $0.1 \mathrm{~nm}$. Specifically, at $\mathrm{L}=50 \mathrm{~nm}$, there is a short-range roughness $\left(\sigma_{50}\right.$ of $0.10 \pm 0.03 \mathrm{~nm}$ and $0.12 \pm 0.01 \mathrm{~nm}$ for the two samples. This reflects the small depressions pointed out in Fig. 3a.

To provide some insight into the origin of the short-range roughness, we measured $\sigma_{50}$ on a $\mathrm{NiAl}(110)$ surface that had been treated, first, by sputtering and annealing. This treatment produced large terraces, 200 to $500 \mathrm{~nm}$ wide, with very low roughness of $\sigma_{50}=$ $0.01 \mathrm{~nm}$. Then the annealed surface was sputtered, whereupon $\sigma_{50}$ increased by almost a factor of 20 , to $0.18 \pm 0.02 \mathrm{~nm}$. This is reasonably close to the corresponding value for the glass, $0.10-0.12 \mathrm{~nm}$, suggesting that the short-range roughness of the glass is due to sputtering. 
At large L (low magnification) $\sigma_{\mathrm{L}}$ of the glass surface increases, reaching values of about $1 \mathrm{~nm}$. At this magnification, roughness is due to the larger depressions that dominate the images in Fig. 3c-f. The physical origin of the larger depressions is unclear. They may be caused by sputtering, or by artifacts of sample preparation such as polishing. The depressions observed on the two length scales are quite similar-compare Fig. 3f with Fig. 3b. Selfsimilar roughness on different length scales has been both predicted and confirmed as a possible result of prolonged surface sputtering $[17,18]$. However, such features were not produced by sputtering the $\mathrm{NiAl}(110)$ sample for lengths of time comparable to the glass.

\subsection{Growth of Ag films}

Figure 5 shows a series of STM images following deposition of Ag at room temperature. Up to a coverage $\left(\theta_{\mathrm{Ag}}\right)$ of $4 \mathrm{ML}$, the presence of an $\mathrm{Ag}$ thin film has no discernible effect on the images. At around $6 \mathrm{ML}$, however, three-dimensional (3D) clusters appear. As $\theta_{\mathrm{Ag}}$ increases to 12 and $20 \mathrm{ML}$, these clusters grow larger. The vertical crosssectional shapes of the clusters at $300 \mathrm{~K}$ are reflected in the line profiles of Fig. $5 \mathrm{~g}$, showing that their widths are much greater than their heights.

The development of the clusters is also reflected in the corresponding value of $\sigma_{\mathrm{L}}$, shown in Fig. 6 as a function of $\theta_{\mathrm{Ag}}$ at $\mathrm{L}=250 \mathrm{~nm}$. Below $4 \mathrm{ML}$, the film has a roughness that is about the same as the initial roughness of the clean metallic glass surface. This suggests that the film conforms to the short-range, sputter-induced roughness of the glass. However, $\sigma_{\mathrm{L}}$ increases abruptly between 4 and $6 \mathrm{ML}$. Hence, a coverage of 4 or $5 \mathrm{ML}$ is a critical thickness $\left(\theta_{\text {crit }}\right)$ below which the film conforms to the substrate, and above which 3D clusters form. 
However, the clusters capture only a fraction of the Ag that is deposited on the surface at $6 \mathrm{ML}$ and above. This conclusion is drawn from the data by estimating the amount of Ag in the clusters from their volumes in the STM images, and from the density of bulk, crystalline Ag. The estimation also rests on knowing the total amount of Ag deposited from the flux, and setting $\theta_{\text {crit }}$ to 4 or $5 \mathrm{ML}$. The calculation shows that the clusters incorporate $<22 \%$ of the Ag that is deposited above $\theta_{\text {crit }}$.

The 3D clusters sometimes appear to consist of several fragments in close proximity. This is particularly obvious in Fig. 5f. This indicates the presence of grain boundaries within the islands, which suggests that the clusters are crystalline. (The crystalline structure is most likely fcc, although this is not confirmed by the present experiments.) Presumably, the grain boundaries are due to growth-induced coalescence, i.e. they form when growing grains meet. Grain boundaries with similar appearance have been observed in STM images of 3D Ag clusters grown on an amorphized $\mathrm{Si}(100)$ surface [19].

\subsection{Temperature dependence of Ag film growth}

In the classic picture of island nucleation and growth far from equilibrium, atoms arrive from the gas phase at a rate $\mathrm{F}$ and become accommodated at the surface as adatoms. This process is irreversible under the conditions of our experiments, i.e. the adatoms cannot re-evaporate into the gas phase. They migrate over the surface at a rate described by a diffusion barrier $E_{d}$, until they are either captured by existing clusters or by combination with other atom(s) to form a new cluster. These new clusters are metastable if they are too small, and can decay back into individual atoms. However, if the cluster survives long enough to grow in size, it becomes more stable and the probability of its growth is greater than the 
probability of decay. The critical island size $\mathrm{i}$ is defined as the size where the addition of just one more atom makes the cluster stable. If one assumes $\mathrm{i}=1$, which means that two adatoms are sufficient to form a stable island, then the island density $\mathrm{N}$ is given by:

$$
N \propto F^{1 / 3} e^{E_{d} / k_{B} T}
$$

Equation (1) shows that, at fixed coverage, $\mathrm{N}$ decreases (island size increases), as deposition temperature increases [20].

The above picture applies to nucleation and growth far from equilibrium. In contrast, for near-equilibrium systems exhibiting SK-growth — notably semiconductor quantum dotsthe island density can show the opposite behavior, i.e. it can decrease with increasing temperature because of entropy [21]. We tested which scenario applies to our system by investigating deposition temperatures in the range 190-300 K.

Figure 7 shows a series of STM images at $\theta_{\mathrm{Ag}}=12 \mathrm{ML}$. The cluster size and density show the expected trends with $\mathrm{T}$ for far-from-equilibrium nucleation. Furthermore, at all four temperatures investigated, clusters are not discernible until the coverage reaches $6 \mathrm{ML}$. Hence, $\theta_{\text {crit }}$ is not T-dependent in this range.

Figure 8 represents the variation of $\ln N$ with reciprocal temperature, at $\theta_{\mathrm{Ag}}=12 \mathrm{ML}$. A linear relationship is expected for homogeneous nucleation at fixed critical nucleus size, $\mathrm{i}$, above which islands are stable. For $\mathrm{i}=1$ (irreversible island formation), the slope is proportional to the surface diffusion barrier, $\mathrm{E}_{\mathrm{d}}$, which in this case would correspond to the barrier for Ag adatoms diffusing on the Ag wetting layer. Clearly, the relationship in Fig. 8 is non-linear. This may suggest a transition to $\mathrm{i}>1$ with increasing $\mathrm{T}$, a phenomenon that is well-known in surface nucleation [22]. The slope of the two data points at lowest $\mathrm{T}$ 
corresponds to $\mathrm{E}_{\mathrm{d}}=0.32 \pm 0.05 \mathrm{eV}$, assuming $\mathrm{i}=1$ in that regime. The uncertainty in $\mathrm{E}_{\mathrm{d}}$ is determined from a propagation-of-error analysis. This value of $E_{d}$ is an upper limit, because one of the points on which it is based (at $\mathrm{T}=240 \mathrm{~K}$ ) may be lowered by its proximity to the bend in the curve in Fig. 8. However, this value of $E_{d}$ is a physically-reasonable limit, because it falls between the known values for $\mathrm{Ag}$ atom diffusion on two low-index $\mathrm{Ag}$ surfaces, i.e. $E_{d}=0.10 \mathrm{eV}$ on $\operatorname{Ag}(111)$, and $E_{d}=0.40-0.45 \mathrm{eV}$ on $\operatorname{Ag}(100)$ [23-25].

A consistency check can be made from a simple formula for the natural crossover variable, $Y$, which describes the transition from irreversible island formation $(i=1)$ where $Y$ $<1$, to reversible island formation $(\mathrm{i}>1)$ where $\mathrm{Y}>>1[25]$ :

$$
Y(T)=\left(\frac{v}{F}\right) \exp \left\lfloor-\left(E_{d}+\frac{3}{2} E_{b}\right) /\left(k_{B} T\right)\right\rfloor
$$

In Eq. (2), $E_{d}$ is the diffusion barrier, $E_{b}$ is the nearest-neighbor pairwise bond energy of about $0.2 \mathrm{eV}$ for $\mathrm{Ag}$ surfaces $[22,25], v=10^{12}$ is the attempt frequency, and $\mathrm{F}$ is the deposition flux, $0.0067 \mathrm{ML} / \mathrm{s}$. The temperature, $\mathrm{T}^{*}$, that describes the transition from $\mathrm{i}=1$ to $\mathrm{i}>1$ is roughly determined from the condition that $\mathrm{Y}\left(\mathrm{T}^{*}\right) \approx 10$. For our choice of $\mathrm{E}_{\mathrm{d}}=0.32$ $\mathrm{eV}$, this condition implies that $\mathrm{T}^{*}=237 \mathrm{~K}$. This is entirely consistent with our assumption above that $\mathrm{i}=1$ at and below $240 \mathrm{~K}$.

From the experimental data, it is informative to evaluate cluster density as a function of coverage at and above $6 \mathrm{ML}$, at constant T. The variation is shown in Table 1 . The cluster density is approximately constant with coverage above $6 \mathrm{ML}$. This suggests that clusters nucleate just when the critical thickness has been exceeded. Beyond that thickness, existing clusters grow but new clusters do not form. At the three lowest temperatures, there are small 
decreases in cluster density with increasing coverage. This can be attributed to growth coalescence.

\section{Discussion}

The data presented above lead us to propose that Ag grows initially in a glass-like wetting layer up to 4-5 monolayers. Above this coverage, crystalline 3D clusters grow, in parallel with the flatter regions. Cluster density remains essentially constant with increasing coverage. Less than $22 \%$ of additional Ag joins the clusters at $\mathrm{T}=190-300 \mathrm{~K}$, with the remainder adding to the flatter layer.

This work deals with the growth of an elemental film on a metallic glass substrate. There are no other studies of such systems reported in the literature, although there have been previous studies of elemental metals on other types of amorphous substrates including carbon [26] and amorphized silicon [19]. In all cases, growth of polycrystalline metal layers was reported, but the existence of a wetting layer was not.

It is well-established that films on crystalline substrates can grow in the so-called Stranski- Krastanov (SK) mode, wherein a film forms a smooth and pseudomorphic, or at least commensurate, wetting layer up to a critical thickness, but it grows as 3D islands above that thickness [27]. The transition is driven by lattice mismatch between film and substrate. The mismatch causes stress accumulation in the growing 2D film, which is strained to match the substrate lattice constant in the wetting layer. Above $\theta_{\text {crit }}$, the clusters adopt a more bulklike atomic structure than in the wetting layer. SK growth has been closely studied in recent years because it yields semiconductor quantum dots on semiconductor surfaces [27]. 
At first glance, it would appear that Ag on this Zr-based glass exhibits a type of SK growth. However, rather than describing the transition to $3 \mathrm{D}$ islands as a result of stress accumulation and lattice mismatch, it is perhaps more appropriate to say that the energy per $\mathrm{Ag}$ atom in the amorphous wetting layer is above that in the crystalline fcc bulk. Thus, for a sufficiently thick film, the energy of the system can be reduced if the upper portion of the film, at least, transforms from the amorphous to the crystalline state. However, the transformation would incur an energy penalty, which is prohibitive for films below a critical thickness.

We do not know of any other example of SK-like film growth, where concurrent 2D and 3D growth has been demonstrated above $\theta_{\text {crit. }}$ A somewhat-related phenomenon can occur in SK growth in semiconductor quantum dot systems, where there is evidence of competition between $2 \mathrm{D}$ and $3 \mathrm{D}$ growth at $\theta_{\text {crit. }}$. In that case, there is a significant barrier to $3 \mathrm{D}$ nucleation. Therefore, the $2 \mathrm{D}$ layer may continue to grow past $\theta_{\text {crit }}$, but eventually $3 \mathrm{D}$ growth begins. When it does, the excess (metastable) material in the wetting layer can transfer to the clusters $[28,29]$. This is distinct from our observation, however, where $2 \mathrm{D}$ and 3D growth appear to occur simultaneously. As mentioned in Section 3c, the conditions of SK growth in semiconductor quantum dot systems are often thought to be near-equilibrium. For a system like ours, the fact that it grows farther-from-equilibrium and kinetics plays a more significant role in determining film structure may explain the competitive growth that we observe. Our observation calls for further examination.

A related issue is the mechanism by which the 3D clusters form. In Sec. 3c, we have treated the data in the context of classical nucleation theory, and the results are physically reasonable - e.g. the trend to lower cluster density with decreasing temperature, and the 
upper limit of $\mathrm{E}_{\mathrm{d}}$. However, in SK growth on crystalline substrates, it is known that clusters can form in two different ways: Nucleation atop a wetting layer, or surface undulations that develop to relieve stress and preserve so-called coherent growth [30]. In an amorphous film, a mechanism might exist in which small 2D crystallites form spontaneously within the amorphous wetting layer, serving as centers for 3D growth, somewhat analogous to coherent growth in crystalline systems. This again calls for further examination.

One might ask whether our surface preparation and ensuing roughness influence the growth mode of Ag. Insight can be gained by comparison with a study of Ag films on an amorphized Si surface [19]. There, the Si surface was prepared by sputtering $\mathrm{Si}(100)$ at room temperature, followed by mild annealing. This procedure is partially analogous to our preparation conditions for the Zr-based glass. Growth of 3D clusters on the Si surface was reported from lowest coverages (roughly $1 \mathrm{ML}$ ) onward at room temperature, which contrasts the observations reported in this paper. This leads us to conclude that SK-like growth on the $\mathrm{Zr}-\mathrm{Ni}-\mathrm{Cu}-\mathrm{Al}$ glass is linked to the chemical nature of the bulk glass. Quite possibly, the wetting layer forms on the $\mathrm{Zr}$-rich glass because the surface energy of $\mathrm{Zr}$ is high relative to $\mathrm{Ag}[31]$.

To conclude, Ag, a bulk-crystalline material, forms a disordered wetting layer on this metallic glass. Thus, it may be possible to chemically modify surfaces of metallic glasses with films of noble metals, while preserving the advantages that accompany the glassy structure. 


\section{Acknowledgments}

The experimental component of this work was supported by the Office of Science, Basic Energy Sciences, Materials Science Division of the U.S. Department of Energy (USDOE) through the Ames Laboratory. Ames Laboratory is operated by Iowa State University of Science and Technology under Contract No. DE-AC02-07CH11358. The theoretical analysis (JWE) was supported by NSF grant CHE-0809472. We thank J. W. Anderegg for the Auger analysis.

\section{References}

[1] A.-L. Barabasi, H.G. Stanley, Fractal Concepts in Surface Growth, Cambridge University Press, Cambridge, 1995.

[2] B. Reinker, M. Moske, K. Samwer, Phys Rev B 56/15 (1997) 9887.

[3] I. Petrov, P.B. Barna, L. Hultman, J.E. Greene, Journal of Vacuum Science \& Technology, A: Vacuum, Surfaces, and Films 21/5, Suppl. (2003) S117.

[4] D. Wang, U. Geyer, S. Schneider, J. Non-Cryst. Solids 221/2,3 (1997) 222.

[5] N. Itoh, W.C. Xu, K. Haraya, Journal of Membrane Science 66/2-3 (1992) 149.

[6] H. Sakaguchi, N. Taniguchi, H. Seri, J. Shiokawa, G. Adachi, J. Appl. Phys. 64/2 (1988) 888 .

[7] J.O. Strom-Olsen, Y. Zhao, D.H. Ryan, Y. Huai, R.W. Cochrane, Journal of the LessCommon Metals 173/1-2 (1991) 922.

[8] S.-I. Yamaura, M. Hasegawa, H. Kimura, A. Inoue, Mater. Trans. 43/10 (2002) 2543.

[9] S.-i. Yamaura, Y. Shimpo, H. Okouchi, M. Nishida, O. Kajita, H. Kimura, A. Inoue, Mater. Trans. 44/9 (2003) 1885. 
[10] J.Y. Kim, P.C. Gibbons, K.F. Kelton, Journal of Alloys and Compounds 266/1-2 (1998) 311.

[11] Note, samples were synthesized at the Materials Preparation Center, Ames Laboratory USDOE, Ames, IA, USA. See: www.mpc.ameslab.gov.

[12] Y. Kawamura, T. Shibata, A. Inoue, T. Masumoto, Acta Mater. 46/1 (1997) 253.

[13] Y. Kawamura, T. Nakamura, H. Kato, H. Mano, A. Inoue, Materials Science \& Engineering, A: Structural Materials: Properties, Microstructure and Processing A304-306 (2001) 674.

[14] L.E. Davis, N.C. MacDonald, P.W. Palmberg, G.E. Riach, R.E. Weber, Handbook of Auger Electron Spectroscopy, Physical Electronics Division, Perkin-Elmer Corporation, Eden Prairie, Minnesota, 1978.

[15] B. Unal, F. Qin, Y. Han, D.J. Liu, D.P. Jing, A.R. Layson, C.J. Jenks, J.W. Evans, P.A. Thiel, Phys Rev B 76/19 (2007).

[16] I. Karakaya, W.T. Thompson, J. Phase Equilib. 13/2 (1992) 143.

[17] J. Krim, I. Heyvaert, C. Vanhaesendonck, Y. Bruynseraede, Phys Rev Lett 70/1 (1993) 57.

[18] S. Habenicht, K.P. Lieb, W. Bolse, U. Geyer, F. Roccaforte, C. Ronning, Nuclear Instruments \& Methods in Physics Research Section B-Beam Interactions with Materials and Atoms 161 (2000) 958.

[19] C. Polop, C. Rosiepen, S. Bleikamp, R. Drese, J. Mayer, A. Dimyati, T. Michely, New Journal of Physics 9 (2007).

[20] J.A. Venables, Philos Mag 27/3 (1973) 697.

[21] M. Meixner, E. Scholl, V.A. Shchukin, D. Bimberg, Phys Rev Lett 8723/23 (2001). 
[22] M.C. Bartelt, L.S. Perkins, J.W. Evans, Surf Sci 344/1-2 (1995) L1193.

[23] H. Brune, K. Brommann, H. Roeder, K. Kern, J. Jacobsen, P. Stoltze, K. Jacobsen, J. Noerskov, Phys. Rev. B: Condens. Matter 52/20 (1995) R14380.

[24] P.A. Thiel, J.W. Evans, Journal of Physical Chemistry B 108/38 (2004) 14428.

[25] J.W. Evans, P.A. Thiel, M.C. Bartelt, Surface Science Reports 61/1-2 (2006) 1.

[26] J.F. Pocza, A. Barna, P.B. Barna, J. Vac. Sci. Technol. 6/4 (1969) 472.

[27] J.A. Venables, Introduction to Surface and Thin Film Processes, 2000.

[28] M. Krishnamurthy, J.S. Drucker, J.A. Venables, J. Appl. Phys. 69/9 (1991) 6461.

[29] Y. Zhang, J. Drucker, J. Appl. Phys. 93/12 (2003) 9583.

[30] C.H. Chiu, Z. Huang, C.T. Poh, Phys Rev Lett 93/13 (2004).

[31] H.L. Skriver, N.M. Rosengaard, Phys Rev B 46/11 (1992) 7157. 


\section{Tables}

Table 1. Cluster density, $\mathrm{N}$, at various temperatures, $\mathrm{T}$, and total coverages, $\theta \mathrm{Ag}$. At $6 \mathrm{ML}$ and $190 \mathrm{~K}$, the clusters cannot be distinguished adequately for counting.

\begin{tabular}{|c|c|c|c|c|}
\hline & $190 \mathrm{~K}$ & $240 \mathrm{~K}$ & $260 \mathrm{~K}$ & $300 \mathrm{~K}$ \\
\hline$\theta_{\mathrm{Ag}}=6 \mathrm{ML}$ & $\mathrm{n} / \mathrm{a}$ & $2900 \pm 141$ & $1180 \pm 30$ & $98.3 \pm 6.9$ \\
\hline $12 \mathrm{ML}$ & $11500 \pm 462$ & $2850 \pm 176$ & $1181 \pm 70$ & $114 \pm 6.1$ \\
\hline $20 \mathrm{ML}$ & $9600 \pm 932$ & $2767 \pm 153$ & $1159 \pm 81$ & $108 \pm 3.6$ \\
\hline
\end{tabular}




\section{Figure captions}

Fig. 1. Auger depth profiles of $\mathrm{Zr}, \mathrm{Cu}, \mathrm{Ni}, \mathrm{Al}, \mathrm{C}$, and $\mathrm{O}$ after the two samples were introduced from air into UHV.

Fig. 2. Scanning electron micrograph of an extruded sample consisting of a mixture of bulk $\mathrm{Ag}$ (light regions) and $\mathrm{Zr}-\mathrm{Ni}-\mathrm{Cu}-\mathrm{Al}-\mathrm{Nb}$ metallic glass (dark regions). The composition of the glass was Zr57Cu15.4Ni12.6Al10 Nb5. The image dimensions are $1175 \mu \mathrm{m} \times 730 \mu \mathrm{m}$.

Fig. 3. STM images of the clean $\mathrm{Zr}-\mathrm{Ni}-\mathrm{Cu}-\mathrm{Al}$ surface at room temperature. The horizontal bar above each frame shows a constant length of $50 \mathrm{~nm}$. (a) $50 \mathrm{~nm} \times 50 \mathrm{~nm}$; (b) 100 $\mathrm{nm} \times 100 \mathrm{~nm}$; (c) $250 \mathrm{~nm} \times 250 \mathrm{~nm}$; (d) $500 \mathrm{~nm} \times 500 \mathrm{~nm}$; (e) $1000 \mathrm{~nm} \times 1000 \mathrm{~nm}$; (f) $2000 \mathrm{~nm} \times 2000 \mathrm{~nm}$. The arrows in (a) point to depressions noted in the text.

Fig. 4. Bulk metallic glass clean surface root mean square (rms) roughness as a function of STM scan length. Solid curve: First sample, sputtered for 40 minutes following introduction from air. Dashed curve: Second sample, sputtered 120 minutes following introduction from air. Surface temperature: $300 \mathrm{~K}$.

Fig. 5. STM images following deposition of Ag coverages at $300 \mathrm{~K}$. Image size: $250 \mathrm{~nm} \times 250$ nm. The Ag coverage in ML is (a) 0; (b) 2; (c) 4; (d) 6; (e) 12; (f) 20. In (g), line profiles of clusters are shown from $6 \mathrm{ML}$ (bottom curve), $12 \mathrm{ML}$ (middle curve), and 20 ML (top curve) are shown.

Fig. 6. Surface roughness (rms) as a function of Ag coverage, following deposition at $300 \mathrm{~K}$. The image size used for analysis is $250 \mathrm{~nm} \times 250 \mathrm{~nm}$. 
Fig. 7. STM images following deposition of $12 \mathrm{ML} \mathrm{Ag}$ at different substrate temperatures. Image size: $250 \mathrm{~nm} \times 250 \mathrm{~nm}$. Temperature: (a) $300 \mathrm{~K}$; (b) $260 \mathrm{~K}$; (c) $240 \mathrm{~K}$; (d) 190 K. The color contrast of the images represents the topographical height.

Fig. 8. Logarithm of island density as a function of reciprocal temperature, at $12 \mathrm{ML} \mathrm{Ag}$ coverage. 
Figures

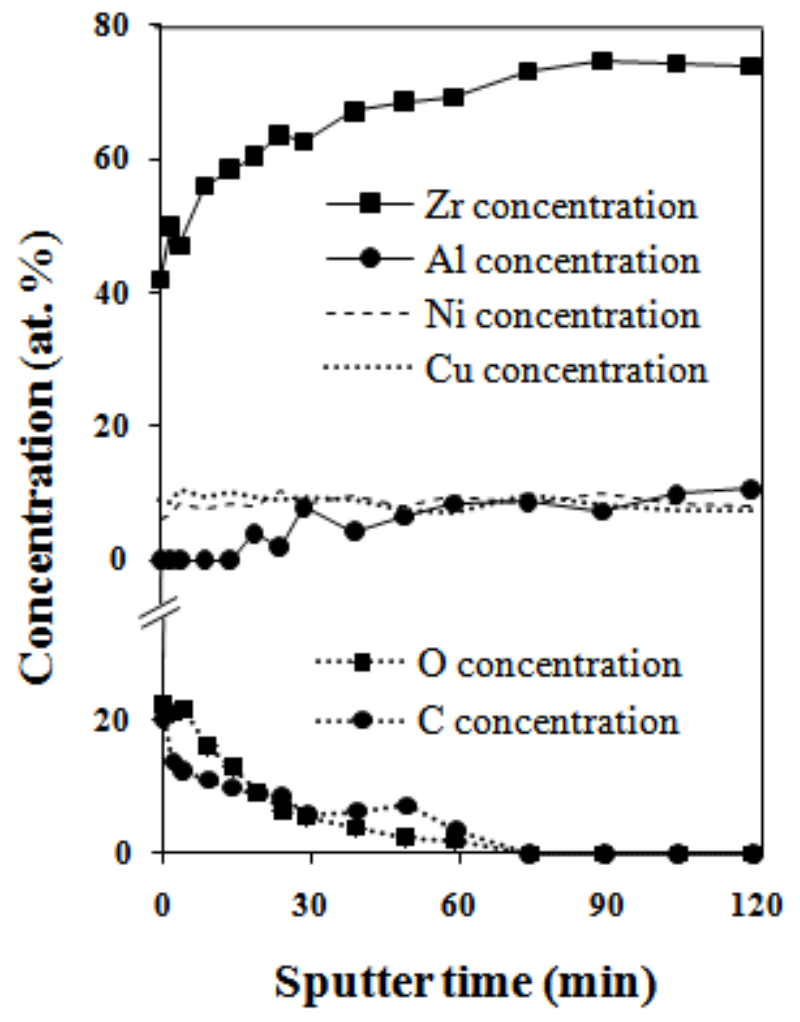

Fig.1 


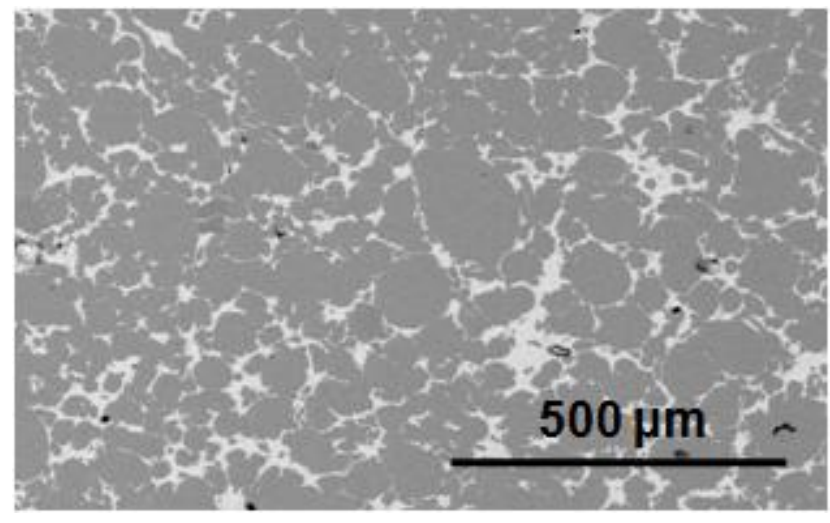

Fig. 2 


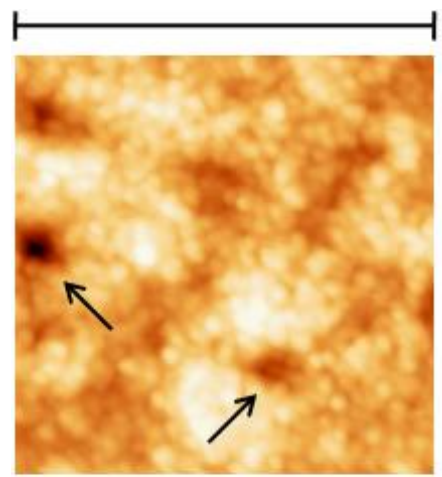

(a)

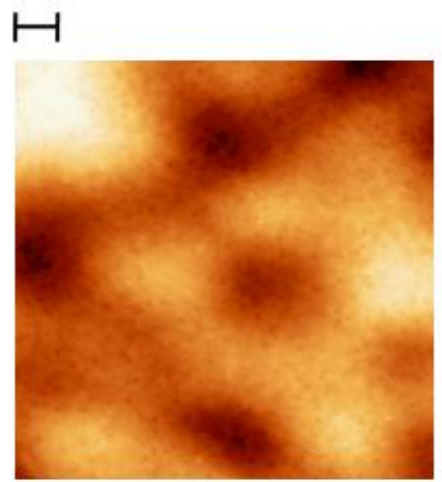

(d)

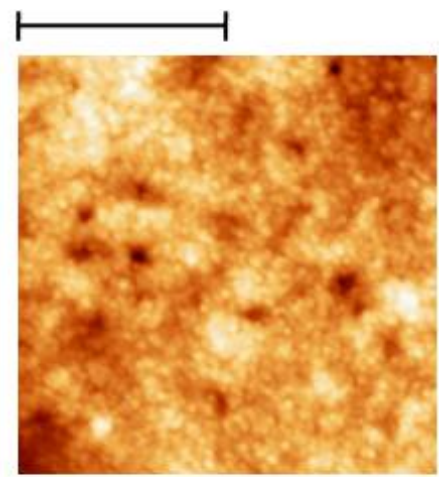

(b)

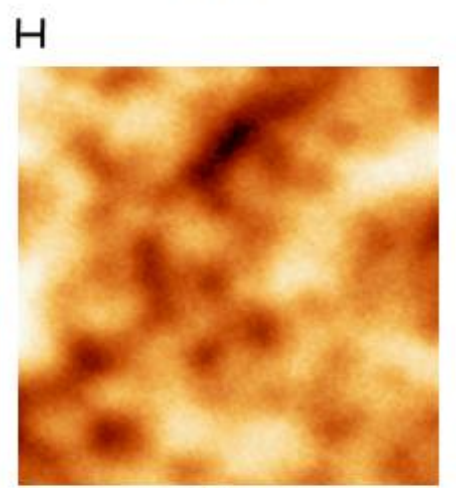

(e)

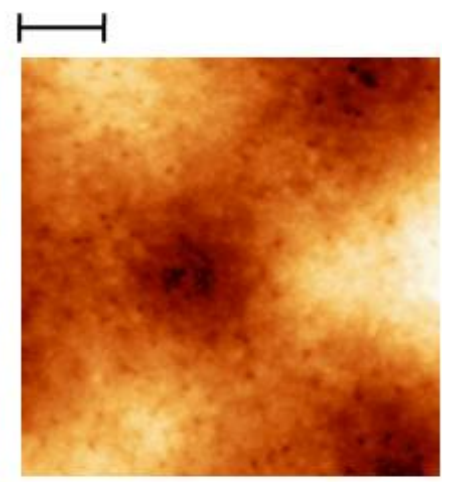

(c)

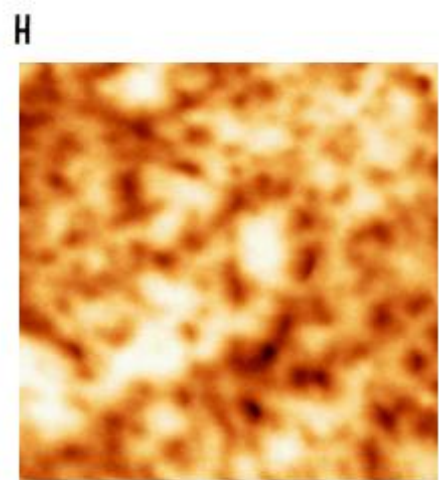

(f)

Fig. 3 


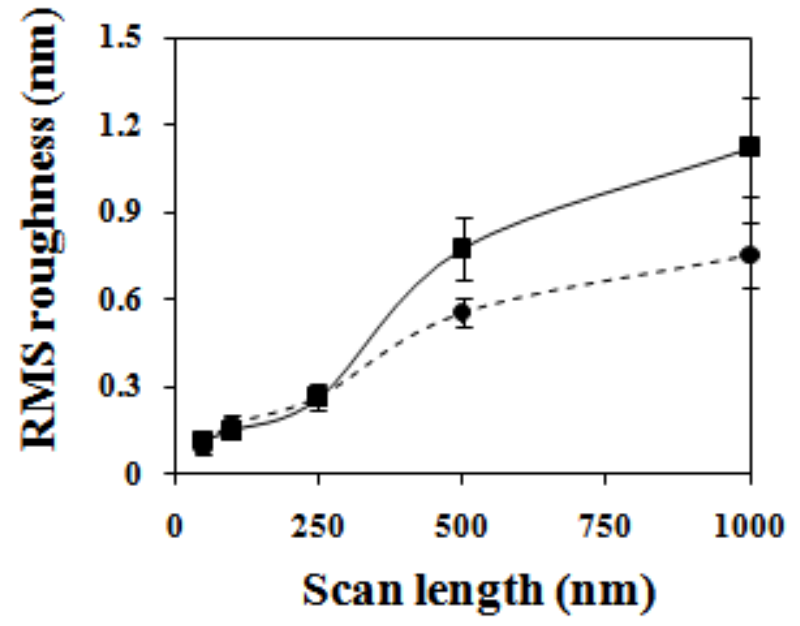

Fig. 4 


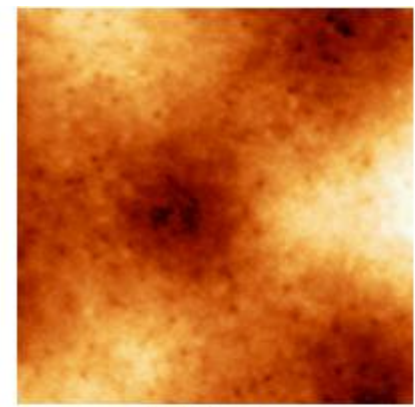

(a) $0 \mathrm{ML}$

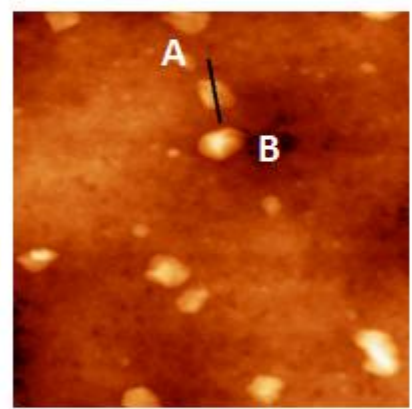

(d) $6 \mathrm{ML}$

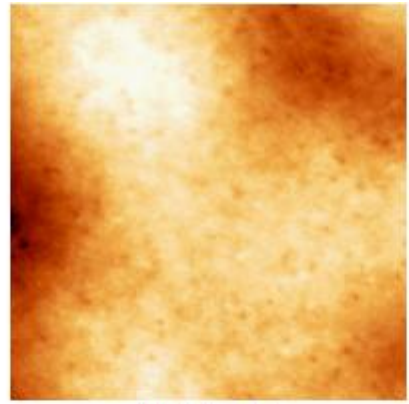

(b) $2 \mathrm{ML}$

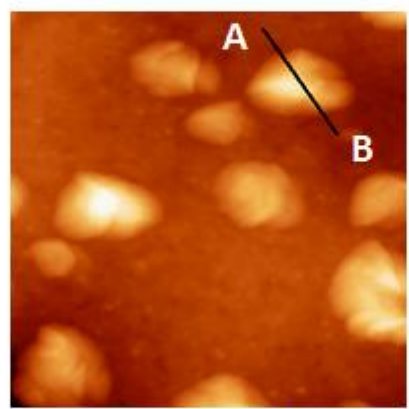

(e) $12 \mathrm{ML}$

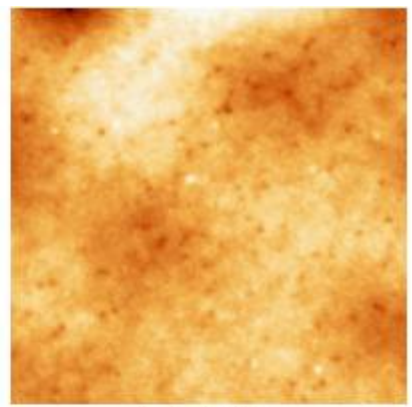

(c) $4 \mathrm{ML}$

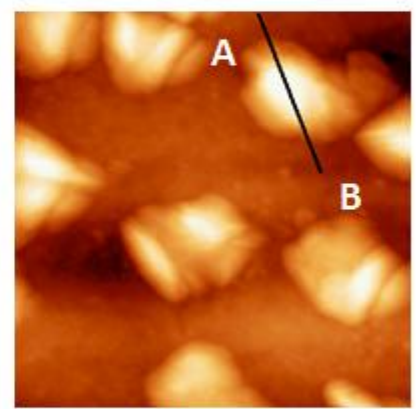

(f) $20 \mathrm{ML}$

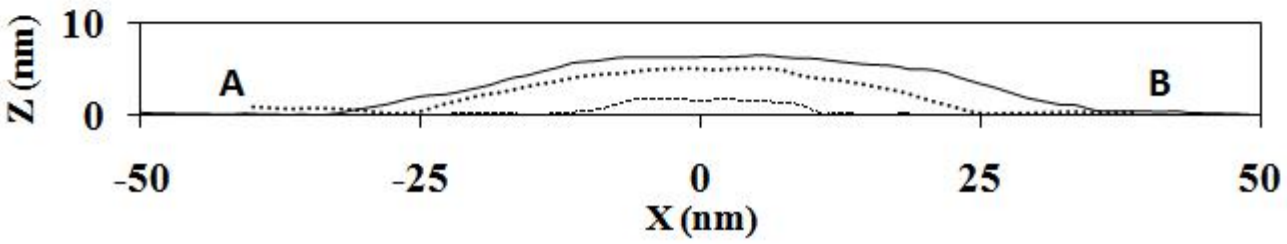

(g)

Fig. 5 


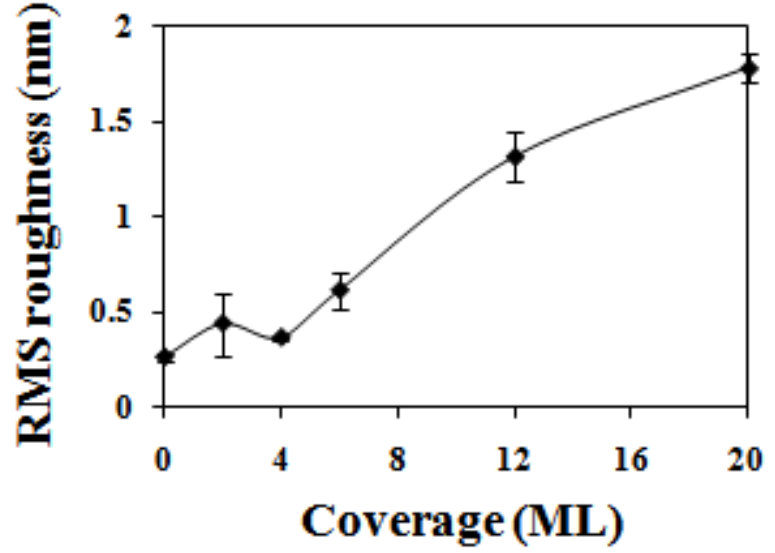

Fig. 6 


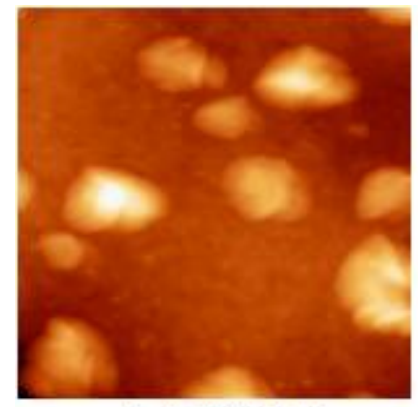

(a) $300 \mathrm{~K}$

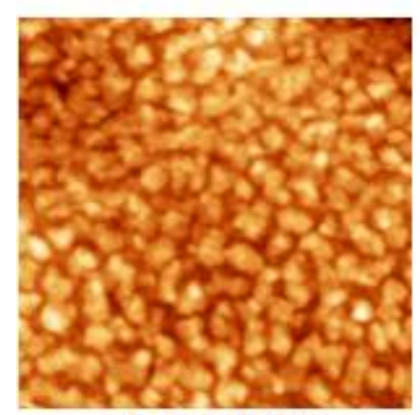

(c) $240 \mathrm{~K}$

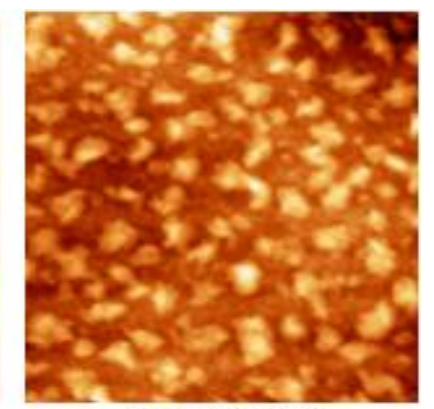

(b) $260 \mathrm{~K}$

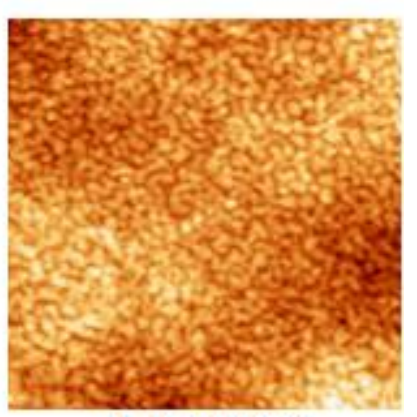

(d) $190 \mathrm{~K}$

Fig. 7 


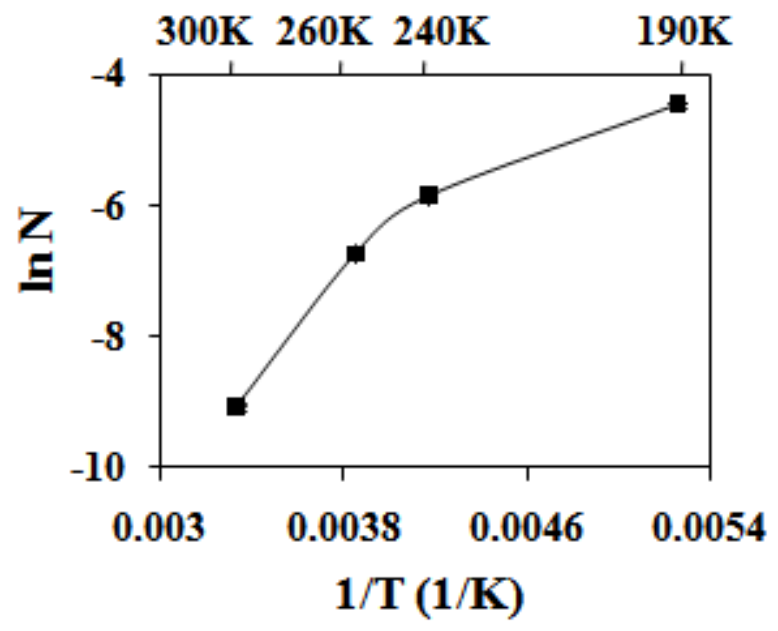

Fig. 8 


\title{
CHAPTER 3. COARSENING AND DECAY OF BILAYER Ag(110) ISLANDS ON NiAI(110): CROSSOVER FROM SMOLUCHOWSKI RIPENING TO OSTWALD RIPENING
}

\begin{abstract}
Scanning tunneling microscopy (STM) studies were performed to analyze the coarsening of bilayer islands. The system was $\mathrm{Ag} / \mathrm{NiAl}(110)$ where a quantum size effect (QSE) stabilizes rectangular bilayer islands. At $185 \mathrm{~K}$ and $205 \mathrm{~K}$, Smoluchowski ripening $(\mathrm{SR}=$ island diffusion and coalescence $)$ dominates, and island diffusion is anisotropic. At $205 \mathrm{~K}$ and $250 \mathrm{~K}$, Ostwald ripening (OR = dissolution of smaller islands by transfer of atoms to larger islands) dominates but "anomalous" one-dimensional island decay is observed rather than conventional shape-preserving decay.
\end{abstract}

\section{Introduction}

Coarsening is a phenomenon in which droplets of a material of one phase separated by another phase (same or a different material) grow larger by mass transport. This process increases the average droplet size at the expense of the number of droplets. There are two main mechanisms of coarsening. They are often referred to as Ostwald ripening [1] and Smoluchowski ripening [2]. The driving force for both mechanisms is the reduction of the free energy associated with reduction of the total area or length of the interfaces between different phases. Understanding coarsening phenomena is of fundamental and technological importance. Particularly, during metal thin film deposition, manipulation of experimental parameters allows control of film morphology. However, coarsening after deposition is 
always a factor that can change film structure since the as-deposited film is generally not in equilibrium.

In epitaxial film growth systems, traditionally, OR is expected to dominate in coarsening. In terms of mass transport medium, there are two scenarios [3]. The first is transport via adatoms (ORA), and the second is transport via vacancies (ORV). In terms of coarsening kinetics, there are two regimes for OR [4,5]. In the first one, the limiting process is terrace diffusion (TD) of the diffusing species. There is no significant barrier for the diffusing species to attach to an island edge. In the second regime, the limiting process is attachment/detachment (AD). The diffusing species encounters a large attachment barrier when attaching to an island edge, or vice-versa for detachment.

For TD-limited OR, the coarsening is strongly affected by the local environment of the islands. On the other hand, for AD-limited OR, the coarsening exhibits mean-field (MF) behavior. In contrast to OR, island diffusion can sometimes be significant allowing SR to dominate [6]. Like in OR, there are also two extreme cases of SR [7]. In case (a), mass transport is achieved by periphery diffusion (PD) of edge adatoms along the island or vacancy step edge. In case (b), adatoms detach from the island or vacancy, diffuse across the terrace and are accommodated by the same island at a different place. This is also called twodimensional (2D) evaporation-condensation (EC).

One can easily discriminate experimentally between OR and SR by following consecutive STM images of the coarsening process. For OR, islands are relatively immobile. With time, small islands get smaller whereas larger islands grow larger. For SR, islands keep their size constant and change in relative positions. When two islands meet, they coalesce and form a bigger island. 
There are many textbook examples of coarsening studies for homoepitaxial systems. $\mathrm{Ag}$ islands on $\mathrm{Ag}(111)$ take the ORA pathway at $300 \mathrm{~K}$ [8-10]. Detailed analyses show this is a TD-limited OR. On the other hand, vacancy pits on $\mathrm{Ag}(111)$ follow the SR pathway at 300 $\mathrm{K}[5,7,11,12]$. Analyses show that this is an EC-mediated SR. In contrast to $\operatorname{Ag}(111)$ homoepitaxy, the $\operatorname{Ag}(100)$ system shows opposite coarsening behaviors. Adatom islands on $\operatorname{Ag}(100)$ follow EC-mediated SR [6,13-15], whereas vacancies on $\operatorname{Ag}(100)$ follow a TDlimited ORV [3,6]. Comparing the coarsening on isotropic surfaces, there also exist studies of homoepitaxial systems on anisotropic surfaces like $\mathrm{Ag}(110), \mathrm{Au}(110)$ and $\mathrm{Pt}(110)$. Results can be found in Ref [16-22]. Contrasting the many studies on homoepitaxy systems, there are less studies on coarsening in heteroepitaxy systems. This is partly due to complications such as complex film morphology caused by lattice mismatch.

In this chapter of the thesis, we present experimental results for coarsening and decay of $\mathrm{Ag}$ bilayer islands on $\mathrm{NiAl}(110)$ surface. The reason for this choice of system is threefold. First of all, even though the bulk structure of the substrate and the film are fundamentally different, there is virtually perfect in-plane lattice matching between fcc $\mathrm{Ag}(110)$ and $\mathrm{CsCl}-$ type $\mathrm{NiAl}(110)\left(\mathrm{a}_{\mathrm{NiAl}}=0.289 \mathrm{~nm}, \mathrm{a}_{\mathrm{Ag}} / \sqrt{ } 2=0.288 \mathrm{~nm}\right)[23]$. Consequently, this system provides an ideal candidate to study heteroepitaxy in the absence of a lateral mismatch strain. Secondly, despite NiAl(110) being an anisotropic surface, DFT analysis shows that Ag adatoms experience a more isotropic diffusion barrier, equal in the two main crystallographic directions, of $0.265 \mathrm{eV}$ [24]. This plays an important role in explaining the coarsening behavior for $\mathrm{Ag}$ islands on $\mathrm{NiAl}(110)$. And last, a previous study of $\mathrm{Ag}$ film growth on $\mathrm{NiAl}(110)$ revealed an initial bilayer growth mode [23]. This growth mode reflects a QSE. The total energy oscillates with the film thickness and even-layer films are more stable than 
odd-layer films when the coverage is less than $10 \mathrm{ML}$ [25]. Therefore, given the unique features mentioned above for this heteroepitaxy system, it can potentially serve as a platform for advancing coarsening theories beyond the ones known for classic homoepitaxial model systems.

A similar coarsening system has been studied by Morgenstern et. al. [18] They have investigated the decay of two-dimensional $\mathrm{Ag}$ islands on the anisotropic $\mathrm{Ag}(110)$ surface using fast scanning STM in the temperature range from $155 \mathrm{~K}$ to $255 \mathrm{~K}$. Two distinct decay behaviors are observed. Below $175 \mathrm{~K}$, no decay is observed. Between $175 \mathrm{~K}$ and $220 \mathrm{~K}$, a quasi-one-dimensional decay is observed. In this mode, the length of the islands decreases, whereas the width of the Ag island stays constant. Above $220 \mathrm{~K}$, the island decays in both directions, and the decay can be described by the well-known OR theory. The transition at $220 \mathrm{~K}$ is accompanied by a fast equilibration of the island shape.

To rationalize the experimental observations, they have performed molecular dynamic calculations of activation energies for various elementary processes relevant for the decay of the $\mathrm{Ag}$ islands. From their energetically reasoning, they are able to elucidate the decay behaviors by identifying the rate limiting atomistic processes [18].

In Sec. 2, we provide some background on our experimental setting and procedure. In Sec. 3, first we briefly describe the general theory of coarsening kinetics for OR and SR, then we present our main experimental observations for SR at lower temperatures and OR at somewhat higher temperatures. At last, we discuss the size scaling of the coarsening rate and the transition from SR to OR. Brief conclusions are provided in Sec. 4. 


\section{Details of experiments}

The experiments were performed in an ultra-high vacuum chamber with a nominal base pressure of $\leq 4 \times 10^{-11}$ Torr. The chamber is equipped with an Omicron variable temperature STM as well as other standard facilities for sample preparation and characterization. The $\mathrm{NiAl}(110)$ single crystal was grown using the Bridgman technique [20]. The sample was cleaned by repeated cycles of 20 min of $\mathrm{Ar}^{+}$sputtering $(1.5 \mathrm{keV}$ at $300 \mathrm{~K})$ followed by annealing to $1250 \mathrm{~K}$ for $1 \mathrm{~h}$. This resulted in a clean surface with broad terraces with an average width of $200 \mathrm{~nm}$. Ag was evaporated from a commercial Mantis e-beam evaporator. The base pressure was below $10^{-10}$ Torr during deposition and the purity of the film was checked by X-ray photoelectron spectroscopy. Flux calibration of the source was achieved by measuring the coverage directly from the STM images in the submonolayer regime.

To prepare the initial configuration of islands for coarsening studies, Ag was deposited onto the $\mathrm{NiAl}(110)$ surface at $185 \mathrm{~K}$ and $205 \mathrm{~K}$. For $250 \mathrm{~K}$ coarsening, Ag was first deposited at $205 \mathrm{~K}$, followed by increasing the surface temperature to $250 \mathrm{~K}$. Care was taken to hold the surface temperature within $\pm 0.5 \mathrm{~K}$. STM measurements were generally started 5 to 10 min after switching off the Ag flux.

To observe the Ag island coarsening process, we recorded consecutive STM images of an area of interest on a terrace typically over a time interval of a few hours. The average time needed to record one STM image is $150 \mathrm{~s}$. We realized that the Ag islands can be perturbed by the scanning process. Therefore we limited close examinations to small islands and sacrificed spatial resolution to minimize perturbation. To exclude the possibility that the coarsening is caused by the scanning process, we first recorded the coarsening behavior of an 
example island, then moved the tip away for over 60 min and came back to observing the same island. We found the island behaves the same way no matter if the tip is scanning it or not. Therefore, we were able to conclude that the coarsening is not caused by the STM tip.

\section{Results and discussion}

\subsection{General theory of coarsening kinetics for OR and SR}

The master equation of analysis of OR is the Gibbs-Thomson (GT) relation [4,26-28]. It gives an expression for the pressure of a vapor that is in equilibrium with its condensed phase. When considering the $\mathrm{OR}$ of $2 \mathrm{D}$ islands on a surface, one can rewrite the relation in terms of the density, $\rho$, of adatoms transporting mass between islands. The relation describes the equilibrium density of adatoms at the edge of an island with radius $\mathrm{R}$ as:

$$
\rho^{\mathrm{eq}}(\mathrm{R}) \approx \rho^{\mathrm{eq}}(\infty) \exp \left[\gamma /\left(\mathrm{k}_{\mathrm{B}} \mathrm{TR}\right)\right]
$$

Here $\mathrm{T}$ is the surface temperature and $\mathrm{k}_{\mathrm{B}}$ is Boltzmann's constant. $\gamma$ is the step energy and $\rho^{\text {eq }}(\infty)$ is the equilibrium density for a straight step $(R=\infty)$. In OR, the diffusion of adatoms is considered to be much faster than the overall coarsening process [29-31]. Therefore, the system can be described by a steady-state solution to the GT relation.

Following the mean-field theory developed by Lifshitz, Slyozov and Wagner $[4,29,30]$, let us consider an island surrounded by an MF environment representing neighboring islands. One can solve a boundary value problem for the steady-state diffusion equation: $0 \approx \partial \rho / \partial \mathrm{t}=\mathrm{D}_{\mathrm{ad}} \nabla^{2} \rho$. Here, $\mathrm{D}_{\mathrm{ad}}$ is the adatom terrace diffusion coefficient. Solution of this diffusion equation gives the decay rate of the island [28]:

$$
\mathrm{dR} / \mathrm{dt} \propto \mathrm{R}^{-\lambda-1}\left(\mathrm{R} / \mathrm{R}_{\mathrm{c}}-1\right)
$$


Here, the critical radius $R_{c}$ provides a measure of the average island size and $\lambda$ is a scaling factor. The integral form of (2) shows the island area decay as:

$$
\mathrm{A} \propto \mathrm{R}^{2} \sim\left(\mathrm{t}_{0}-\mathrm{t}\right)^{2 /(\lambda+2)}
$$

Here $t=t_{0}$ when $R=0$. The values for the scaling factor $\lambda$ of 1 and 0 have been predicted [3] for the prediction for the TD-limited and AD-limited OR regimes mentioned in Sec. 1. From this, $\mathrm{A} \sim\left(\mathrm{t}_{0}-\mathrm{t}\right)^{2 / 3}$ for TD-limited $\mathrm{OR}$ and $\mathrm{A} \sim\left(\mathrm{t}_{0}-\mathrm{t}\right)$ for AD-limited OR. Another commonly used expression for this power law behavior of OR has the form:

$$
\mathrm{A} \propto \tau^{2 \beta}
$$

where $\tau=\mathrm{t}_{0}-\mathrm{t}$ is called the decay time and $\beta=(\lambda+2)^{-1}$.

The motion of a 2D island is caused by the thermal fluctuation of film material leading to motion of the center of mass of the island. The migration can be described by the island diffusion coefficient D. It has been shown from both theory [32-35] and experiments $[6,7,13,15,36]$ that the diffusion coefficient should depend on the island size via:

$$
\mathrm{D} \propto \mathrm{d}^{-\beta}
$$

Here $\mathrm{d}$ is the diameter of the island and $\beta$ is a scaling factor. For coarsening study on an anisotropic surface like $\mathrm{NiAl}(110)$, it is more relevant to write the size-scaling law as:

$$
D \propto A^{-\beta / 2}
$$

Note that the $\beta$ in Eq. 4 is different from the one used above in Eq. 3b. The island diffusion coefficient also has a temperature-dependent part. Thus, the whole form can be written as:

$$
\mathrm{D} \propto \exp \left(\mathrm{E}_{\mathrm{diff}} / \mathrm{k}_{\mathrm{B}} \mathrm{T}\right)(\mathrm{A})^{-\beta / 2}
$$

Here $\mathrm{E}_{\text {diff }}$ is the island diffusion barrier.

The island diffusion coefficient can be defined via the Einstein relation $\left\langle(\Delta \mathrm{x})^{2}\right\rangle=$ $2 \mathrm{D} \Delta \mathrm{t}$. Here $<(\Delta \mathrm{x})^{2}>$ is the mean square displacement (MSD) of a diffusing island obtained 
from statistical measurements. Thus D can be extracted from the experimental value of $<(\Delta \mathrm{x})^{2}>/ \Delta \mathrm{t}$. Derivations in Ref $[9,18]$ show D scales with the island size as D $\sim(\mathrm{A})^{-3 / 2}$ for PD-mediated SR and D A for EC-mediated SR. That is $\beta=3$ and 2 for PD-mediated SR and EC-mediated SR respectively.

\subsection{Experimental observations for SR}

In this subsection, we present our STM observations for $\mathrm{Ag} / \mathrm{NiAl}(110)$

Smoluchowski ripening behavior. At $185 \mathrm{~K}$, initial Ag island distribution is created by deposition of 0.15 bilayer Ag with a flux of $1.0 \times 10^{-2} \mathrm{ML} / \mathrm{s}$. Note here a bilayer is defined as two layers of a perfect $\operatorname{Ag}(110)$ film [23]. Fig. 1a and 1b show the first and last of a series of STM images scanning the same area over a time interval of $116 \mathrm{~min}$. Fig. 1c displays the relative motion of the center of mass of the two islands in Fig. 1a and 1b for the first 28 min. The coordinates for the center of mass of the islands are generated automatically by the software WSxM [37]. We always record the relative displacement of two islands in the same STM image due to the absence of a reference point which can be considered as not moving during the island coarsening process. As one can see in Fig. 1c, the relative motion extends over $14 \mathrm{NiAl}(110)$ unit cells along the [001] direction. The direction perpendicular to [001] corresponds to [-110] and the relative motion along this direction is less than two $\mathrm{NiAl}(110)$ unit cells. Superficially, Fig. 1c represents a quasi one-dimensional (1D) random walk with fluctuations perpendicular to the random walk direction. To verify this, one should realize that a random walk must obey the Einstein relation $<(\Delta \mathrm{x})^{2}>=2 \mathrm{D} \Delta \mathrm{t}$ [7]. The MSD should have linear time dependence with the diffusion coefficient being half of the slope. And 
indeed, our experimental data shows the linear relationship between the MSD and the $\Delta \mathrm{t}$ as seen in Fig. 1d.

As an aside, we should note that since we are analyzing the relative displacement of two islands, the diffusion coefficient $\mathrm{D}$ is actually the sum of two diffusion coefficients for the two individual islands. And the Einstein relation should be written as: $\left\langle(\Delta \mathrm{x})^{2}\right\rangle=2\left(\mathrm{D}_{1}+\right.$ $\left.D_{2}\right) \Delta t$. However, from Eq. $4 \mathrm{~b}$, we see that islands of the same size have the same diffusion coefficient $\left(D_{1}=D_{2}=D / 2\right)$. Therefore, theoretically, if one analyzes the relative motion of two islands of the same size, individual island diffusion coefficient can be obtained.

Similar coarsening behavior is observed for $205 \mathrm{~K} \mathrm{Ag}$ deposition. Fig. $2 \mathrm{a}$ and $2 \mathrm{~b}$ show the first and last of a series of consecutive STM images scanning the same area over a time interval of $88 \mathrm{~min}$. Fig. 1c displays the relative motion of the center of mass of the two islands in Fig. 2a and $2 \mathrm{~b}$ for the first $31 \mathrm{~min}$. As we can see, the two islands diffuse cross the terrace and finally coalesce and become one single island. The area of the island in Fig. $2 b$ is the same as the total area of islands in Fig. 2a. However, one feature in this SR is different than the one at $185 \mathrm{~K}$. At $205 \mathrm{~K}$, the relative motion along the [-110] direction (horizontal direction in the STM images) appears to be more active. This is evidenced in Fig. 2c, the trajectory spreads out more widely compared to Fig. 1c along the [-110] direction. Also in Fig. 2d, the mean square displacement along [-110] shows a linear time dependence indicating the relative motion along this direction is a random walk as well as the relative motion along [001]. However, the MSD vs. $\Delta t$ plot for [001] has a larger slope compared to that for [-110]. This indicates that the diffusion coefficient for [001] motion is larger, which means the relative motion along this direction is more active. 


\subsection{Experimental observations for OR}

In this subsection, we present the experimental observations for $\mathrm{Ag} / \mathrm{NiAl}(110)$ Ostwald ripening behavior. OR is first observed at $205 \mathrm{~K}$. Initial Ag island distribution is created by deposition of 0.15 bilayer Ag with a flux of $1.0 \times 10^{-2} \mathrm{ML} / \mathrm{s}$ at $205 \mathrm{~K}$. We find a 1D decay mode, contrary to conventional shape-preserving predictions. For this anisotropic island decay mode, the length of the islands decreases, whereas the corresponding width stays constant until the island has almost disappeared (see Fig. 3).

Fig. 4 shows this 1D decay mode more clearly. As illustrated in Fig. 4a, the area of the island decreases continuously during the island decay. The area decay is caused by length decrease, not width, as shown in Fig. 4c. Double logarithmic plot (Fig. 3b) reveals that the area of the island follows a simple power law behavior similar to that given by Eq. 3b. The exponent is determined from the slope of the linear fit, which is found to be 0.39 . Fig. $4 \mathrm{~d}$ shows the double logarithmic plot for island length and width. The line for the width is almost horizontal as expected from Fig. $4 \mathrm{c}$ with an exponent value of 0.01 . The line for the length has a similar exponent value as the area line. This again indicates that OR at $205 \mathrm{~K}$ follows a 1D decay mode.

Ostwald ripening is also observed at a higher temperature. After Ag deposition at 205 $\mathrm{K}\left(0.15\right.$ bilayer coverage with a flux of $\left.1.0 \times 10^{-2} \mathrm{ML} / \mathrm{s}\right)$, the surface temperature is increased to $250 \mathrm{~K}$. At this temperature, Ag islands follow the same 1D decay mode in which the length decreases whereas the width stays constant (see Fig. 3). The island decay at $250 \mathrm{~K}$ is also illustrated in Fig. 5 more clearly. As shown in Fig. 5a and 5c the area and length decrease continuously whereas the width stays constant during island decay. Double logarithmic plot of area vs. decay time $\tau$ (Fig. $5 b$ ) reveals the power law behavior similar to 
that seen in $205 \mathrm{~K}$ island decay. The exponent is found to be 0.55 . Double logarithmic plot for island length and width (Fig. 5d) also confirms the 1D decay mode with a zero slope for the width line and the same exponent for the length line as the area line.

From Fig. 4a and 5a, we can see that the island area decays nonlinearly with time. From Fig. $4 \mathrm{~b}$ and $5 \mathrm{~b}$, we also see that the slopes for the lines in the double logarithmic plots are smaller than 1 . Especially for the one at $250 \mathrm{~K}$, the $2 \beta$ value is close to the TD-limited OR prediction with a value of $2 / 3$. From these observations, we conclude that the $\mathrm{Ag}$ bilayer islands on $\mathrm{NiAl}(110)$ follow a TD-limited Ostwald ripening at $205 \mathrm{~K}$ and $250 \mathrm{~K}$. Another

piece of evidence for $\mathrm{TD}$-limited $\mathrm{OR}$ for $\mathrm{Ag} / \mathrm{NiAl}(110)$ lies in the curve crossing of evolution of island size vs. time for an ensemble of islands (see Fig. 5e and 5f). For TD-limited OR, island decay depends strongly on their local environment. This is different than AD-limited OR where decay of islands is determined entirely by average island size. Therefore, following the evolution of size vs. time for a group of islands, one finds occasional curve crossings. This is observed in our $250 \mathrm{~K}$ experiment and illustrated in Fig. 5e. There are also curve crossings in the double logarithmic plot of size vs. decay time. As shown in Fig. 5f, this corresponds to the situation where two decaying islands, at a certain time, have the same size. However, they have different local environments and thus different decay rates.

\subsection{Transition from SR to OR}

In this subsection, we discuss the transition from Smoluchowski ripening to Ostwald ripening of $\mathrm{Ag}$ bilayer islands on $\mathrm{NiAl}(110)$ as temperature increases. To begin, it is convenient to define a coarsening rate, $\mathrm{K}=\mathrm{dR}_{\mathrm{av}} / \mathrm{dt}$ with $\mathrm{R}_{\mathrm{av}}$ being the average island radius. 
One can express the coarsening rate $\mathrm{K}$ in terms of coarsening energetics and average island radius as $[3,38]$ :

$$
\mathrm{K}=\mathrm{dR}_{\mathrm{av}} / \mathrm{dt} \approx v \exp \left[-\mathrm{E}_{\mathrm{eff}} /\left(\mathrm{k}_{\mathrm{B}} \mathrm{T}\right)\right]\left(\mathrm{R}_{\mathrm{av}}\right)^{-\mathrm{m}}
$$

Here, $v$ is a prefactor, and $\mathrm{E}_{\mathrm{eff}}$ is the effective barrier. Different values of $\mathrm{m}$ have also been assigned to different coarsening pathways. For AD-limited OR, $\mathrm{m}=1$; For TD-limited OR, $\mathrm{m}=2$; For EC-mediated SR, $\mathrm{m}=3$ and for PD-mediated SR, $\mathrm{m}=4[3,16]$. Therefore, as we can see in Eq. 6, the factors that determine the value of $\mathrm{K}$ are the energetics (i.e., the effective barrier $\mathrm{E}_{\text {eff }}$ ) and the size-scaling (i.e., the exponent $\mathrm{m}$ ). Different coarsening pathways have different forms of $\mathrm{E}_{\text {eff }}$ depending on the detailed atomic processes during coarsening. Temperature $\mathrm{T}$ and deposition flux $\mathrm{F}$ determine the average island radius $\mathrm{R}_{\mathrm{av}}$. The overall coarsening should follow the pathway with the largest value of $\mathrm{K}$ for a certain system under a certain experimental condition. And naturally, changing the system and/or the experimental condition may cause a different pathway to win and thus change the overall coarsening behavior. For instance, Ag adatom islands change their coarsening pathway from SR on $\mathrm{Ag}(100)$ surface to $\mathrm{OR}$ on $\mathrm{Ag}(111)$ surface at $300 \mathrm{~K}$ [3]. And in our $\mathrm{Ag} / \mathrm{NiAl}(110)$ system, the island coarsening pathway changes from SR to OR as temperature increases.

Next, we will focus on the island size-scaling to discuss the transition from SR to OR for our $\mathrm{Ag} / \mathrm{NiAl}(110)$ heteroepitaxy system. As shown in Eq. 6, the coarsening rate scales differently with average island radius for different pathways due to different $\mathrm{m}$ values. Therefore, a double logarithmic plot of $\mathrm{K}$ as a function of $\mathrm{R}_{\mathrm{av}}$ for different pathways in a specific system will show lines of different slopes (see Fig. 6). Considering only the effect of size-scaling, the slope for PD-mediated SR is the largest while the slope for AD-limited OR is the smallest. This indicates SR will win on the left hand side of the plot (small $R_{a v}$ ) and OR 
will win on the right hand side of the plot (large $\mathrm{R}_{\mathrm{av})}$. For far from equilibrium film growth systems, island size decreases with decreasing temperature. Therefore, the left hand side of Fig. 6 corresponds to low temperatures and the right hand side of Fig. 6 corresponds to relatively higher temperatures. The point where two lines intersect reflects the transition point.

One should note that the above argument ignores the effect of energetics $\mathrm{E}_{\mathrm{eff}}$ and prefactor $v$. Appropriate values for $\mathrm{E}_{\mathrm{eff}}$ and for $v$ are necessary to determine the dominant pathway. Effective barrier $\mathrm{E}_{\mathrm{eff}}$ for different pathways can be theoretically calculated once the detailed atomic processes are learned. For our system, the calculation of $\mathrm{E}_{\mathrm{eff}}$ is more complex than the classic systems mentioned in the introduction due to the fact that Ag bilayer island formation is triggered by a QSE. An appropriate selection of the prefactor $v$ is also important for this analysis. Given the appropriate values for $\mathrm{E}_{\mathrm{eff}}$ and for $v$, one can determine the dominant pathway for any given temperature. In general, there exists a transition temperature $\mathrm{T}_{\mathrm{x}}$ such that $\mathrm{SR}$ dominates for $\mathrm{T}<\mathrm{T}_{\mathrm{x}}$ and $\mathrm{OR}$ dominates for $\mathrm{T}>\mathrm{T}_{\mathrm{x}}[3]$. For our system, experimental observations show that the transition temperature appears to be $205 \mathrm{~K}$ where both SR and OR are observed.

\section{Conclusion}

In summary, we have presented the first study of coarsening and decay of bilayer islands. The system was $\mathrm{Ag}$ on $\mathrm{NiAl}(110)$ in the temperature range from $185 \mathrm{~K}$ to $250 \mathrm{~K}$. The coarsening behavior, has some similarities to that seen in the $\operatorname{Ag}(110)$ homoepitaxial system. [18] At $185 \mathrm{~K}$ and $205 \mathrm{~K}$, coarsening of Ag islands follows a Smoluchowski ripening pathway. At $205 \mathrm{~K}$ and $250 \mathrm{~K}$, the terrace diffusion limited Ostwald ripening dominants. The 
experimental observed temperature for the transition from SR to OR is $205 \mathrm{~K}$. The SR exhibits anisotropic island diffusion and the OR exhibits 1D decay of island length while keeping the corresponding island width constant.

\section{References}

[1] W. Ostwald, Lehrbuch der allgemeinen Chemie, Leipzig, Germany, 1896.

[2] M. Smoluchowski, Physikalische Zeitschrift 17 (1916) 557 and 585.

[3] P.A. Thiel, M. Shen, D.-J. Liu, J.W. Evans, Journal of Physical Chemistry C 113/13 (2009) 5047.

[4] M. Zinke-Allmang, L.C. Feldman, M.H. Grabow, Surface Science Reports 16/8 (1992) 377.

[5] K. Morgenstern, Phys. Status Solidi B 242/4 (2005) 773.

[6] J.M. Wen, J.W. Evans, M.C. Bartelt, J.W. Burnett, P.A. Thiel, Phys Rev Lett 76/4 (1996) 652.

[7] K. Morgenstern, G. Rosenfeld, B. Poelsema, G. Comsa, Phys Rev Lett 74/11 (1995) 2058.

[8] K. Morgenstern, G. Rosenfeld, G. Comsa, Phys Rev Lett 76/12 (1996) 2113.

[9] K. Morgenstern, G. Rosenfeld, E. Laegsgaard, F. Besenbacher, G. Comsa, Phys Rev Lett 80/3 (1998) 556.

[10] K. Morgenstern, G. Rosenfeld, G. Comsa, Surf Sci 441/2-3 (1999) 289.

[11] G. Rosenfeld, K. Morgenstern, M. Esser, G. Comsa, Appl. Phys. A: Mater. Sci. Process. $69 / 5$ (1999) 489. 
[12] D.C. Schlosser, K. Morgenstern, L.K. Verheij, G. Rosenfeld, F. Besenbacher, G. Comsa, Surf Sci 465/1-2 (2000) 19.

[13] J.M. Wen, S.L. Chang, J.W. Burnett, J.W. Evans, P.A. Thiel, Phys Rev Lett 73/19 (1994) 2591.

[14] C.R. Stoldt, C.J. Jenks, P.A. Thiel, A.M. Cadilhe, J.W. Evans, Journal of Chemical Physics 111/11 (1999) 5157.

[15] W.W. Pai, A.K. Swan, Z. Zhang, J.F. Wendelken, Phys Rev Lett 79/17 (1997) 3210.

[16] K. Morgenstern, E. Laegsgaard, F. Besenbacher, Phys. Rev. B: Condens. Matter $66 / 11(2002) 115408 / 1$

[17] K. Morgenstern, E. Laegsgaard, F. Besenbacher, Phys Rev Lett 86/25 (2001) 5739.

[18] K. Morgenstern, E. Laegsgaard, I. Stensgaard, F. Besenbacher, Phys Rev Lett 83/8 (1999) 1613.

[19] Y. Yao, P. Ebert, M. Li, Z. Zhang, E.G. Wang, Phys. Rev. B: Condens. Matter 66/4 (2002) 041407/1.

[20] M.J. Rost, R. van Gastel, J.W.M. Frenken, Phys Rev Lett 84/9 (2000) 1966.

[21] M.J. Rost, S.B. van Albada, J.W.M. Frenken, Phys Rev Lett 86/26, Pt. 1 (2001) 5938.

[22] T.R. Linderoth, S. Horch, L. Petersen, E. Laegsgaard, I. Stensgaard, F. Besenbacher, New Journal of Physics 7/13 (2005) 13.

[23] B. Unal, F. Qin, Y. Han, D.J. Liu, D.P. Jing, A.R. Layson, C.J. Jenks, J.W. Evans, P.A. Thiel, Phys Rev B 76/19 (2007).

[24] Y. Han, B. Unal, D. Jing, F. Qin, C.J. Jenks, D.-J. Liu, P.A. Thiel, J.W. Evans, Phys. Rev. B: Condens. Matter 81/11 (2010) 115462/1.

[25] Y. Han, D.-J. Liu, Phys. Rev. B: Condens. Matter 80/15 (2009) 155404/1. 
[26] Single crystal synthesisized at the Materials Preparation Center, Ames Laboratory, US-DOE. See www.mpc.ameslab.gov.

[27] L. Ratke, P.W. Voorhees, Coarsening and Growth: Ostwald Ripening in Materials Processes, Springer, Berlin, 2001.

[28] W.K. Burton, N. Cabrera, E.C. Grank, Philos. Trans. Roy. Soc. (London) A243 (1951) 299.

[29] F. Hausser, A. Voigt, Phys. Rev. B: Condens. Matter 72/3 (2005) 035437/1.

[30] I.M. Lifshitz, V.V. Slyozov, Journal of Physics and Chemistry of Solids 19/1-2 (1961) 35 .

[31] C. Wagner, Zeitschrift Fur Elektrochemie 65/7-8 (1961) 581.

[32] B.K. Chakraverty, Journal of Physics and Chemistry of Solids 28/12 (1967) 2401.

[33] S.V. Khare, N.C. Barlelt, T.L. Einstein, Phys Rev Lett 75/11 (1995) 2148.

[34] G. Mills, T.R. Mattsson, L. Mollnitz, H. Metiu, Journal of Chemical Physics 111/18 (1999) 8639.

[35] D.S. Scholl, R.T. Skodje, Phys Rev Lett 75/17 (1995) 3158.

[36] J. Heinonen, I. Koponen, J. Merikoski, T. Ala-Nissila, Phys Rev Lett 82/13 (1999) 2733.

[37] A.M. Cadilhe, C.R. Stoldt, C.J. Jenks, P.A. Thiel, J.W. Evans, Phys. Rev. B: Condens. Matter 61/7 (2000) 4910.

[38] I. Horcas, R. Fernandez, J.M. Gomez-Rodriguez, J. Colchero, J. Gomez-Herrero, A.M. Baro, Rev Sci Instrum 78/1 (2007).

[39] M. Shen, J.M. Wen, C.J. Jenks, P.A. Thiel, D.-J. Liu, J.W. Evans, Phys. Rev. B: Condens. Matter 75/24 (2007) 245409/1. 


\section{Figure captions}

Fig. 1 The (a) first and (b) last of a series of STM images taken at $185 \mathrm{~K}$ over a time interval of 116 min. Image size: $8 \times 12 \mathrm{~nm} 2$. Tip bias: $+0.5 \mathrm{~V}$, tunneling current: $0.5 \mathrm{nA}$. (c) shows relative displacement of the center of mass of the two islands shown in (a) for the first 28 min. The grid represents $\mathrm{NiAl}(110)$ surface lattice. (d) shows relative mean square displacement along [001] direction as a function of elapsed time $\Delta \mathrm{t}$ for the relative motion shown in (c).

Fig. 2 The (a) first and (b) last of a series of STM images taken at $205 \mathrm{~K}$ over a time interval of 88 min. Image size: $15 \times 20 \mathrm{~nm} 2$. Tip bias: $-1 \mathrm{~V}$, tunneling current: $0.5 \mathrm{nA}$. (c) shows relative displacement of the center of mass of the two islands shown in (a) for the first $31 \mathrm{~min}$. The grid represents $\operatorname{NiAl(110)}$ surface lattice. (d) shows relative mean square displacement along [001] direction (red) and [-110] direction (blue) as a function of elapsed time $\Delta \mathrm{t}$ for the relative motion shown in (c).

Fig. 3 Series of STM images recording the 1D decay of Ag islands on NiAl(110) at $205 \mathrm{~K}$ (upper row: $20 \times 20 \mathrm{~nm} 2$ ) and $250 \mathrm{~K}$ (lower row: $11 \times 18 \mathrm{~nm} 2$ ). Tip bias: $-1 \mathrm{~V}$, tunneling current: $0.5 \mathrm{nA}$.

Fig. 4 OR at $205 \mathrm{~K}$ : (a) shows island area decay as a function of time. (b) double logarithmic plot of area vs. time with linear fit. $2 \beta$ value is the slope of the line. (c) evolution of island length (red) and width (blue). (d) double logarithmic plot of island length (red) and width (blue) vs. time with linear fit.

Fig. 5 OR at $250 \mathrm{~K}$ : (a) shows island area decay as a function of time. (b) double logarithmic plot of area vs. time with linear fit. $2 \beta$ value is the slope of the line. (c) evolution of island length (red) and width (blue). (d) double logarithmic plot of island length (red) 
and width (blue) vs. time with linear fit. (e) shows area decay for a group of five islands. (f) shows double logarithmic plot for the five islands shown in (e).

Fig. 6 Comparison of coarsening rates of different pathways vs. mean island size. The blue line is for PD-mediated SR, the green line is for EC-mediated SR, the red line is for TD-limited OR and the orange line is for AD-limited OR. Note that this figure does not correspond to any specific coarsening system. This figure is only a schematic illustration of the coarsening size-scaling behavior. The slope of the lines in this figure does not take the energetics and prefactor into account. 
Figures
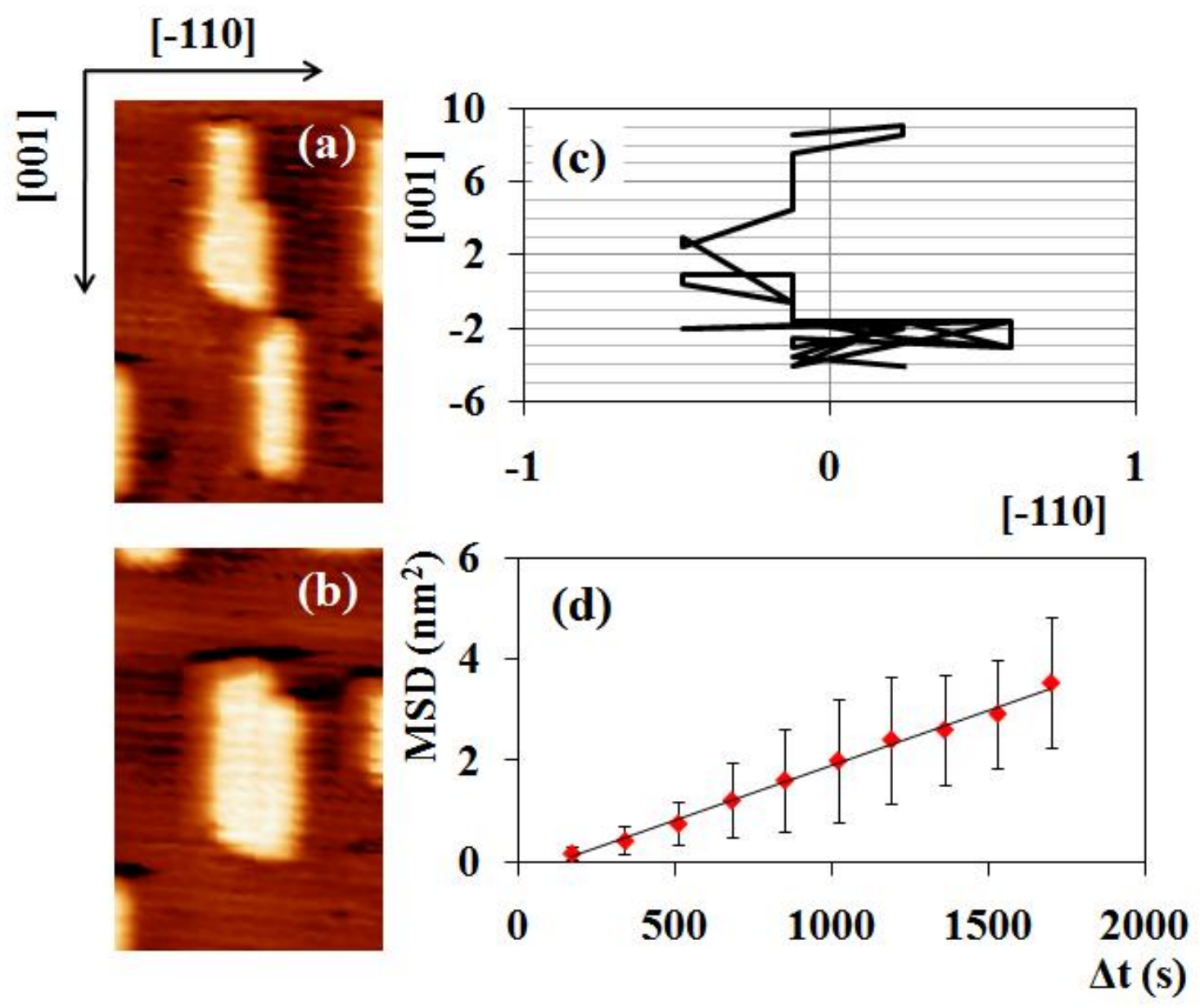

Fig. 1 

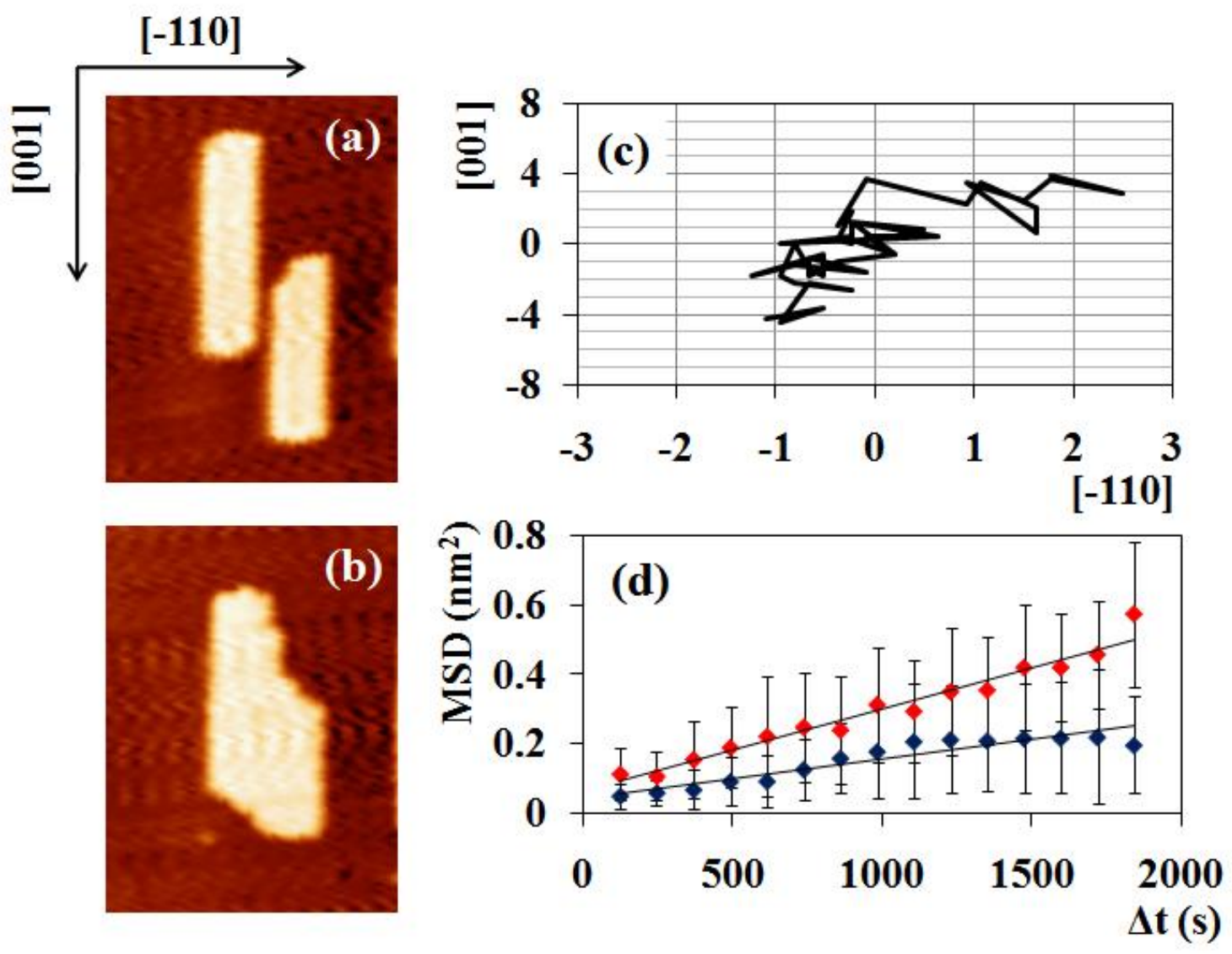

Fig. 2 


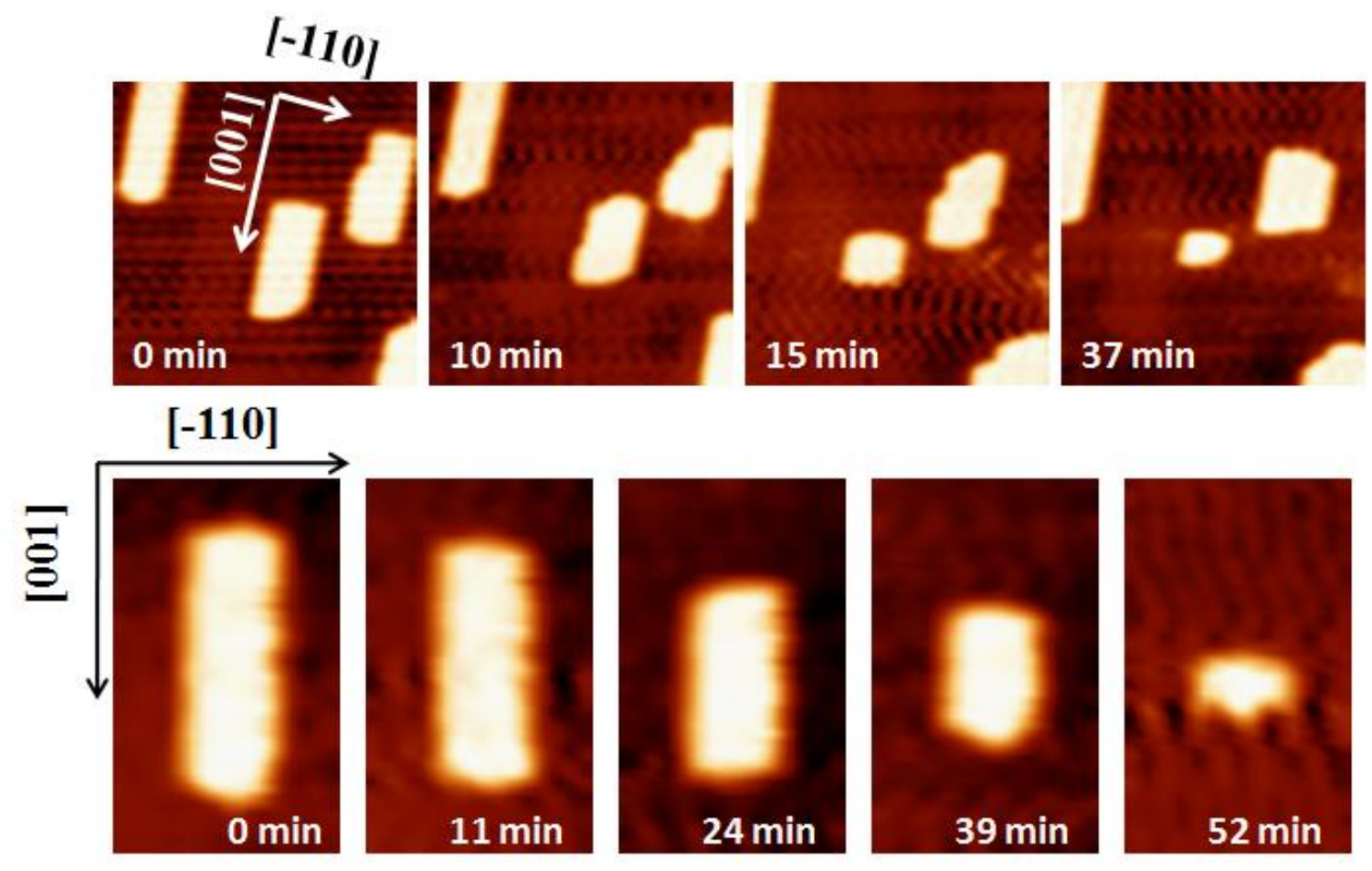

Fig. 3 

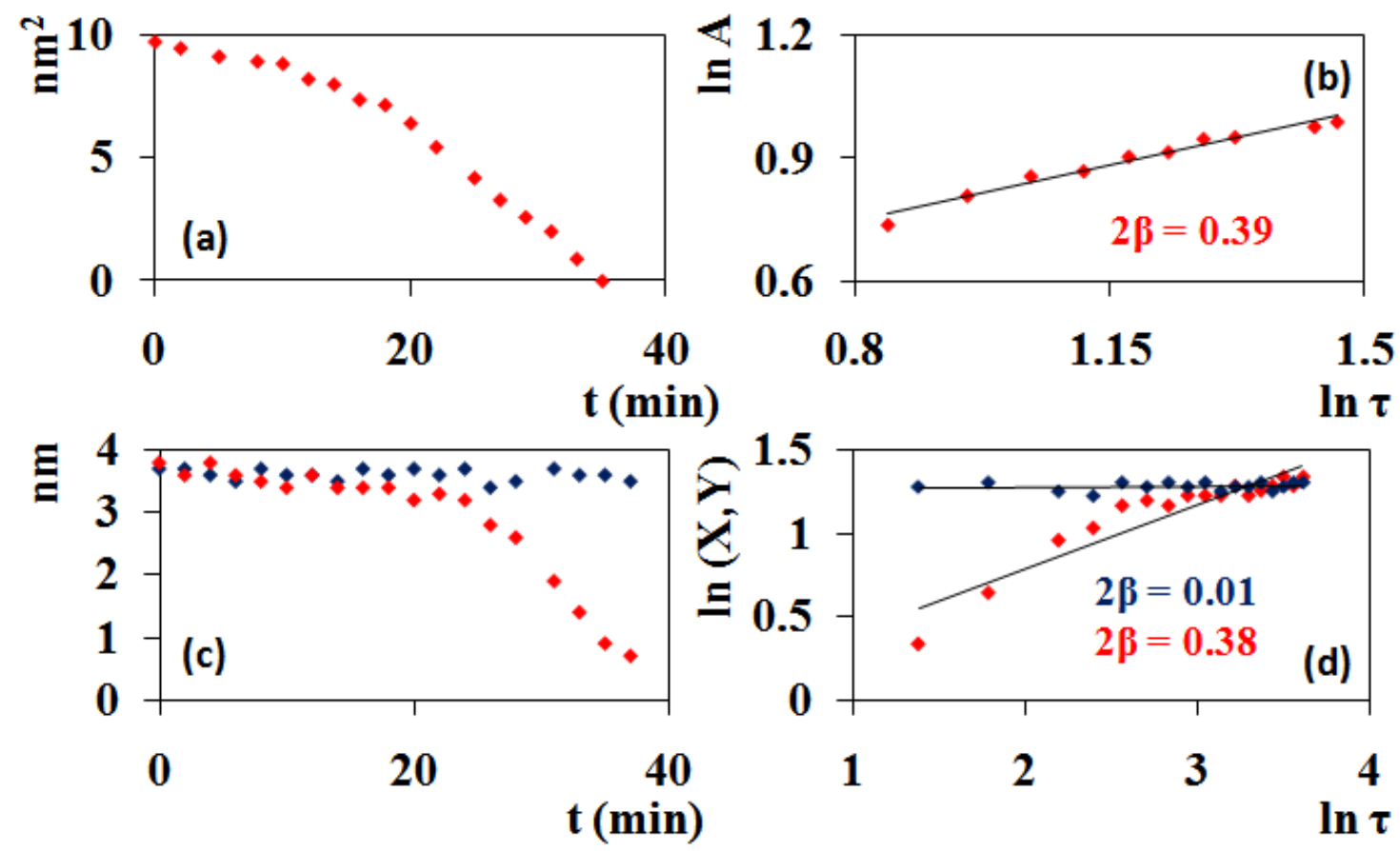

Fig. 4 

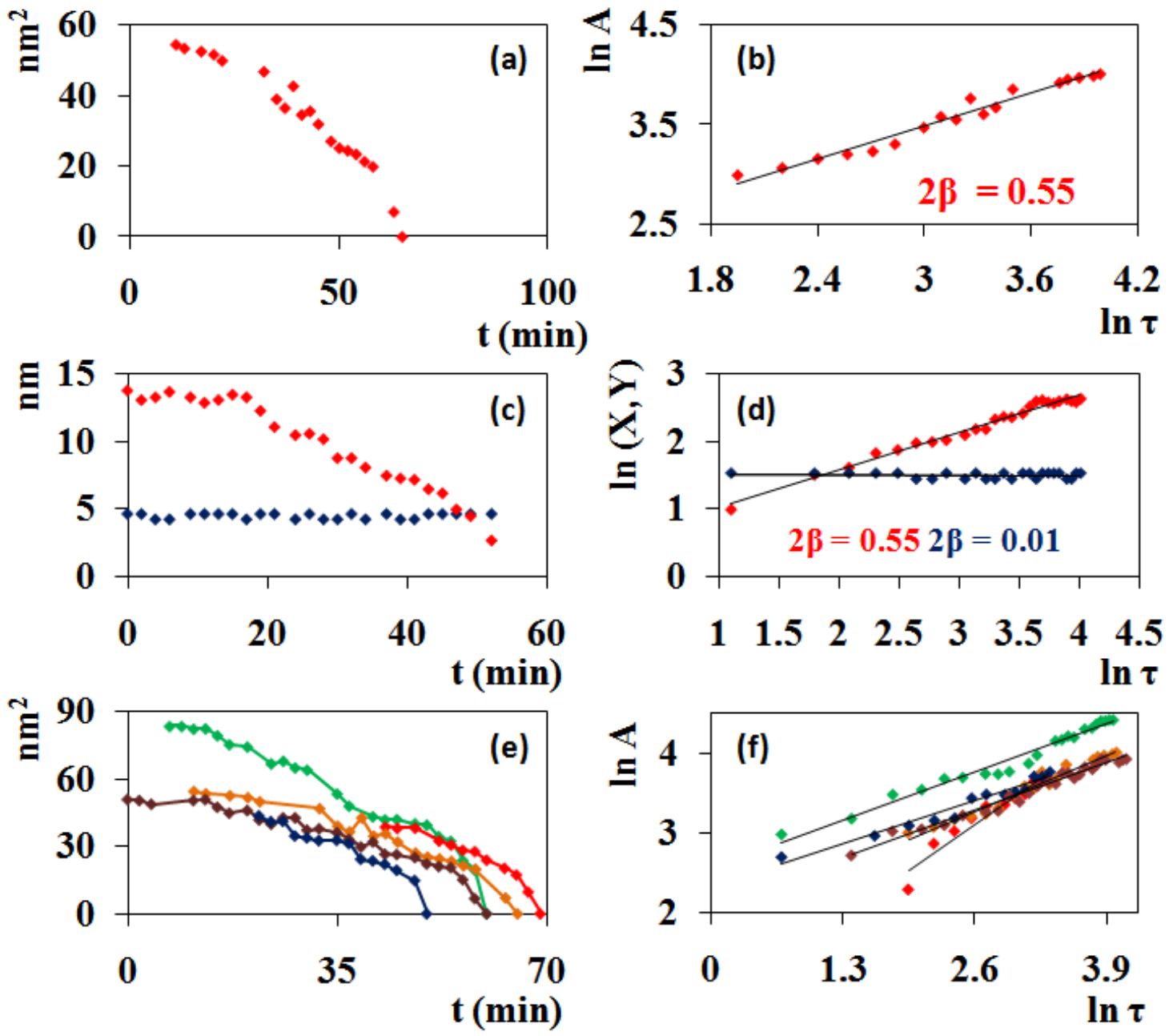

Fig. 5 


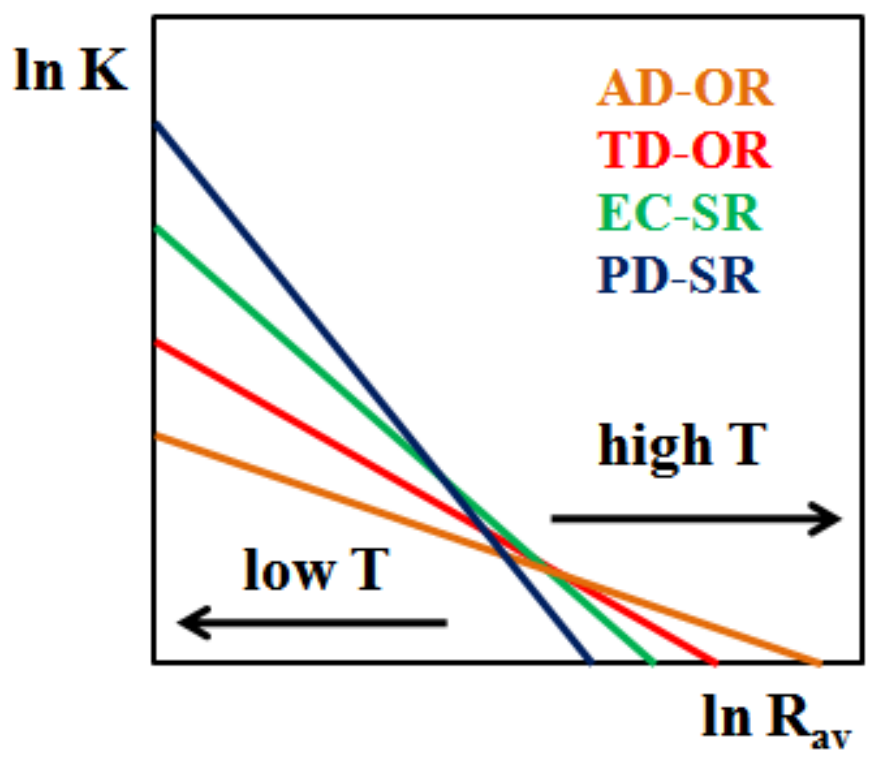

Fig. 6 


\section{CHAPTER 4. LOW-ENERGY ELECTRON DIFFRACTION STUDY OF Ag THIN FILM GROWTH ON NiAI(110)}

\section{Introduction}

Previously, we reported a study of Ag islands formed by nucleation and growth on NiAl(110).[1] Using scanning tunneling microscopy (STM), we determined that these islands can be highly elongated along the $\mathrm{NiAl}$ [001] direction, i.e. their long edge is parallel to the $\mathrm{Ni}-\mathrm{Ni}$ bridge sites. The elongation increases with deposition temperature in the range from 130 to $300 \mathrm{~K}$. An example of elongated islands at $300 \mathrm{~K}$ is shown in Fig. 1A.

We further determined that the $\mathrm{Ag}$ islands consist of bilayers of $\operatorname{Ag}(110)$, based upon the measured heights of these islands.[1] The driving force for bilayer growth instead of monolayer growth is revealed by DFT, which shows a Quantum Size Effect (QSE), i.e. electronic stabilization of particular island heights. Growth of the (110) orientation is undoubtedly promoted also by the near-perfect lattice match between $\mathrm{Ag}(110)$ and $\mathrm{NiAl}(110)$ parallel to the surface plane. However, strain must exist because of the crystallographic mismatch: $\mathrm{Ag}$ is fcc, while $\mathrm{NiAl}$ is the $\mathrm{CsCl}$ structure. Indeed, $\mathrm{STM}$ shows that the $\mathrm{Ag}$ islands contain internal structure in the form of linear depressions and linear protrusions. These features again align with the NiAl [001] direction. They are attributed to strain in the film. Examples of the depressions are shown in Fig. 1B and 1C.

However, DFT simulations showed that other structures are energetically competitive with the (110) bilayer.[2] The competing structures can be called "square-hex-type," since they consist of local square and hexagonal motifs. The simplest involves squares and hexagons in a 1:1 ratio, and another viable structure has these two units in a 1:2 ratio. The 
calculated energy difference between the best square-hex-type structure and the (110) BL is small, $0.02 \mathrm{eV} /$ atom,[2] and it is not clear that this difference is significant. Also, kinetics may favor the (110) bilayer during growth.[3]

This provides one motivation for the current study. Within the first few layers of Ag, our objective is to distinguish between the (110) structure and the square-hex-type (SH) structure, based simply upon their different surface unit cells. The surface unit cells will be determined using low-energy electron diffraction (LEED). If the Ag film grows as a (110) BL, then to first order, the LEED pattern should remain unchanged by Ag growth. On the other hand, the surface unit cell of any SH is much larger, so its development should cause extra spots to appear in the diffraction pattern. Fig. 2A-D illustrate the real-space and reciprocal-space lattices of $\mathrm{NiAl}(110), \mathrm{Ag}(110)$, and two SH-ML overlayers. Relevant surface lattice constants are given in Table 1.

A second motivation exists, and is related to Ag multilayer growth. Based on Ag island heights measured with STM, bilayer growth reverts to monolayer growth after completion of about 3 (110) BLs. After this point, the height of each new layer is consistent with it being either $\operatorname{Ag}(111)$ or $\operatorname{Ag}(100)$. In favor of $\operatorname{Ag}(111)$, there are many reports in the literature of multilayer $\mathrm{Ag}$ films exhibiting a (111) texture, $[1,4,5]$ even after growth at room temperature. The (111) is energetically preferred because it is close-packed and hence has lowest surface energy. Our second objective is to determine whether LEED can detect evidence of either of the (111) or (100) surface orientations in multilayer growth for this system, $\mathrm{Ag} / \mathrm{NiAl}(110)$. 


\section{Experimental details}

Experiments were carried out in two different ultrahigh vacuum chambers, using two different NiAl samples. The majority of the experiments were done in the so-called LEED chamber (which was equipped only for LEED), but some experiments were also done in the so-called STM chamber (which was equipped both for STM and LEED). Coverages of Ag in the STM chamber were determined from STM images. Coverages of Ag in the LEED chamber were determined by comparing LEED patterns between the two systems. Fig. 3 compares LEED patterns obtained from the two systems. Fig. 3A shows LEED patterns for the clean surface at two energies, and Fig. 3B shows LEED patterns after Ag deposition at various energies. In the type of comparison shown in Fig. 3B, i.e. comparing the STM chamber pattern for known coverage with the LEED chamber patterns for various deposition time, the best comparison (although not perfect) was obtained for the pair of data sets shown. This was used to establish that a coverage of $3 \mathrm{ML}$ was achieved in the LEED chamber after 15 seconds of deposition. It follows that the flux was $0.2 \mathrm{ML} / \mathrm{s}$ in the LEED chamber, and this is used in determining all coverages reported in the following text. Note that in the STM chamber, the Ag flux was $0.007 \mathrm{ML} / \mathrm{s}$. Thus, the deposition flux used in the LEED chamber is 30 times higher than the one used in the STM chamber. In epitaxial film growth systems, deposition with a higher flux may result in a poorly-ordered film or a greater density of dislocations than using a lower flux. Furthermore, postdeposition annealing is known to help develop film ordering. Therefore, to minimize the deposition flux effects, we subsequently anneal the Ag films prepared in the LEED chamber at $400 \mathrm{~K}$ for $30 \mathrm{~min}$. We then compare the LEED patterns for the annealed films in the LEED chamber to the reference pattern to get more precise flux estimation. 
The LEED chamber was equipped with a home-built electron-beam evaporator described in the Appendix, LEED optics, sputter gun, and quadrupole mass spectrometer. During the course of these experiments, the base pressure was typically $1 \times 10^{-10}$ Torr. The LEED optics were only operational at beam energies of 200 to $300 \mathrm{eV}$, except in one instance when a beam voltage as low as $90 \mathrm{eV}$ was obtained.

The sample used in the LEED chamber had not been used for other experiments. It was cleaned between $\mathrm{Ag}$ depositions by sputtering at $1.5 \mathrm{keV}$ at normal incidence for 15 minutes, annealing at $1150 \mathrm{~K}$ for 1 hour. A good LEED pattern with sharp diffraction spots, as seen in Fig. 3A, was taken as evidence of a clean surface.

In the LEED chamber, patterns were acquired after cooling the sample to $110 \mathrm{~K}$. Typically, about 15 minutes elapsed between Ag deposition at $300 \mathrm{~K}$ and the beginning of LEED experiments. If the sample was annealed after Ag deposition, it was held at $400 \mathrm{~K}$ for 30 minutes. In that case, about an hour elapsed between $\mathrm{Ag}$ deposition at $300 \mathrm{~K}$ and the beginning of LEED experiments.

More experimental details about the evaporator and the cleanliness of the Ag film are provided in the Appendix to this chapter.

\section{Experimental results and interpretation}

Figs. 4-6 show LEED patterns following Ag adsorption in the LEED chamber. Each figure shows LEED patterns at a fixed energy, so comparisons can be made between different coverages within the figure. In all cases, Ag was deposited at $300 \mathrm{~K}$. If an image is marked "anneal" then the sample was subsequently annealed to $400 \mathrm{~K}$ for 30 minutes. 
Images for other beam voltages at $5 \mathrm{~V}$ intervals from $200 \mathrm{~V}$ to $300 \mathrm{~V}$ are also available but not shown here.

From visual inspection of the images within each figure, the positions of the diffraction spots and hence the reciprocal-space unit cell of the surface appears to be unchanged by Ag deposition, up to about $12 \mathrm{ML}$. The constancy of the pattern confirms that Ag grows as a (110) BL rather than SH-ML structure. The intensities of the spots change with Ag coverage, which is natural since the nature and local environment of the scatterers changes drastically upon Ag deposition. This changes the intensity-voltage variation of diffraction spots, but not their presence.

It also appears that annealing does not have any significant effect on the patterns. This is consistent with our previous suggestion[1] that there is no alloying in this system, at least up to $400 \mathrm{~K}$. Alloying between Ag and transition metals at surfaces is known to occur, but typically requires temperatures of $500 \mathrm{~K}$ or above.[6-12]

A closer inspection reveals two interesting things. First, the positions of the diffraction spots do change very slightly with Ag deposition. In fact, the unit cell in reciprocal space is a few tenths of a percent larger after deposition of $\mathrm{Ag}$, than for the clean NiAl(110) surface. Consider first the data from the LEED chamber, shown in Fig. 7. Fig. 7A shows the reciprocal-space lattice aspect ratio and Fig. 7B shows the relative lattice constants. From this dataset, the Ag unit cell is $0.25 \pm 0.09 \%$ smaller in real space than the $\mathrm{NiAl}(110)$ surface unit cell. Consider next the data from the STM chamber, shown in Fig. 8. From this, the $\mathrm{Ag}$ unit cell is $0.36 \pm 0.13 \%$ smaller in real space. These two values are identical within experimental error and although small, they are not zero. The value is remarkably reproducible, considering that it was measured on two different sets of LEED 
optics, two different NiAl samples, and two different Ag evaporators. Based upon the lattice constants for bulk Ag and bulk NiAl, the difference should be $0.24 \%$, which agrees very well with the observations. This suggests that the tiny lateral lattice mismatch is relieved via dislocations.

The second interesting feature relates to these dislocations. Between $3 \mathrm{ML}$ and 24 ML, extra spots appear in the diffraction patterns. These are shown in Fig. 9A-C, for different Ag coverages, regions of the diffraction pattern, and beam energies. Conditions are chosen for which the additional spots are clearest. These extra diffraction spots correspond to an extra periodicity in the [-110] direction of $1.21 \pm 0.04 \mathrm{~nm}$, in real space. This is the same as the spacing between some of the dislocation lines shown in Fig. 1B. With STM, we had previously observed that the dislocation lines - the depressions - were mostly spaced by either 0.8 or $1.2 \mathrm{~nm}$. In fact, a model for the $1.2 \mathrm{~nm}$ dislocations was generated on the basis of DFT, and is reproduced in Fig. 10. It is not clear why the $1.2 \mathrm{~nm}$ dislocations are more evident in the LEED patterns, or more prevalent on the surface, than are the $0.8 \mathrm{~nm}$ dislocations. It is also not clear why they were observed already in the first BL in the previous STM studies, but in LEED they only appear after Ag coverage reaches $1.5 \mathrm{BL}$. In general, it seems that features appear in the LEED data at slightly higher coverages than in the STM data. Perhaps this represents a systematic error in coverage calibration between the two chambers, or perhaps the sensitivity of LEED is somewhat deficient.

Finally, the LEED patterns of Figs. 4-6 show that the (111) structure emerges at high coverage. The hexagonal pattern is illustrated by the hexagons drawn for illustration in Figs. 4R and 6R. This hexagonal pattern is commensurate with the substrate in [001] direction, leading to the real-space model of Fig. 11. Note that the $(-2,2)$ spot of the hexagonal pattern 
overlaps with the (-2,2) spot of the substrate. At $250 \mathrm{eV}$ (Fig. 6), this spot is essentially absent for the substrate but it is intense for the $\mathrm{Ag}(111)$ film. Therefore it can be used to determine the coverage at which the (111) pattern emerges. This occurs between 9 and 12 ML. Compare, for instance, Fig. 6H (9 ML) with Fig. 6I (12 ML), where the key diffraction spot is encircled. This means that the (111) is detected in LEED at 4.5 to 6 BL, slightly higher than the 4 BL that would have been predicted from the previous STM study of step heights.

\section{Conclusions}

LEED indicates that, up to about $6 \mathrm{BL}$ (12 ML), the Ag film adopts the (110) structure on lattice matched $\mathrm{NiAl}(110)$ surface, supporting the previous assignment based upon island heights measured in STM.[1] Starting at 4.5 to $6 \mathrm{BL},(111)$ diffraction pattern is detected. This is also in agreement with previous STM study. Careful examinations of the LEED patterns reveal the slight difference in lattice constants between bulk Ag and bulk NiAl.

\section{References}

[1] B. Unal, F. Qin, Y. Han, D.J. Liu, D.P. Jing, A.R. Layson, C.J. Jenks, J.W. Evans, P.A. Thiel, Phys Rev B 76/19 (2007).

[2] Y. Han, B. Unal, D. Jing, F. Qin, C.J. Jenks, D.-J. Liu, P.A. Thiel, J.W. Evans, Phys. Rev. B: Condens. Matter 81/11 (2010) 115462/1.

[3] Y. Han, B. Unal, F. Qin, D. Jing, C.J. Jenks, D.-J. Liu, P.A. Thiel, J.W. Evans, Phys Rev Lett 100/11 (2008) 116105/1. 
[4] C. Deisl, E. Bertel, M. Buergener, G. Meister, A. Goldmann, Phys. Rev. B: Condens. Matter 72/15 (2005) 155433/1.

[5] E. Bauer, H. Poppa, G. Todd, P.R. Davis, J Appl Phys 48/9 (1977) 3773.

[6] A.K. Santra, C.N.R. Rao, Appl Surf Sci 84/4 (1995) 347.

[7] A. Bzowski, M. Kuhn, T.K. Sham, K.H. Tan, Journal of Vacuum Science \& Technology, A Vacuum, Surfaces, and Films 12/4, Pt. 2 (1994) 1790.

[8] R. Fischer, T.H. Fauster, Surface Review and Letters 3/5 \& 6 (1996) 1783.

[9] V.R.R. Medicherla, W. Drube, Appl Surf Sci 256/2 (2009) 376.

[10] D. Pacile, C.R. Ast, M. Papagno, C. Da Silva, L. Moreschini, M. Falub, A.P. Seitsonen, M. Grioni, Phys. Rev. B: Condens. Matter 73/24 (2006) 245429/1.

[11] P. Schieffer, C. Krembel, M.C. Hanf, G. Gewinner, Y. Gauthier, Phys. Rev. B: Condens. Matter 65/23 (2002) 235427/1.

[12] C. Tegenkamp, M. Michailov, J. Wollschlager, H. Pfnur, Appl Surf Sci 151/1-2 (1999) 40. 


\section{Tables}

Table 1: Real-space surface lattice constants for NiAl(110), Ag(110), Ag square-hex and Ag square-hex-hex overlayers.

\begin{tabular}{|c|c|c|c|c|}
\hline Cryst. direction & NiAl(110) & $\operatorname{Ag}(110)$ & Square-Hex & Square-Hex-Hex \\
\hline$[001]$ & $0.289 \mathrm{~nm}$ & $0.288 \mathrm{~nm}$ & $0.289 \mathrm{~nm}$ & $0.289 \mathrm{~nm}$ \\
\hline$[-110]$ & $0.409 \mathrm{~nm}$ & $0.408 \mathrm{~nm}$ & $0.818 \mathrm{~nm}$ & $1.227 \mathrm{~nm}$ \\
\hline
\end{tabular}




\section{Figure captions}

Fig. 1: STM data for Ag deposition on $\mathrm{NiAl}(110)$ at $300 \mathrm{~K}$. Tip bias: $+1 \mathrm{~V}$, tunneling current: $0.5 \mathrm{nA}$. (A) shows Ag form elongated islands. The long edge is parallel to the $\mathrm{NiAl}$ [001] direction. Image size: $500 \times 500 \mathrm{~nm} 2$. (B) Example of ripples in the Ag island. Depressions with period $0.8 \mathrm{~nm}$. Image size: $15 \times 15 \mathrm{~nm} 2$. (C) Depressions with period of $1.2 \mathrm{~nm}$. Image size: $15 \times 15 \mathrm{~nm} 2$.

Fig. 2: Schematic illustration for the real-space and reciprocal-space lattices of (A) NiAl(110), (B) Ag(110), (C) Square-hex and (D) Square-hex-hex overlayers. Substrate surface unit cell is marked by black rectangle and overlayer unit cells are marked by red rectangles.

Fig. 3: (A) shows LEED patterns for the clean surface from LEED chamber and STM chamber at $210 \mathrm{~V}$ and $230 \mathrm{~V}$. (B) shows LEED patterns after Ag deposition.

Fig. 4: LEED patterns at $210 \mathrm{~V}$ following Ag adsorption in the LEED chamber. Upper-right corner of each image shows the Ag coverage. 0 ML means NiAl(110) clean surface. If an image is marked "anneal" then the sample was subsequently annealed to $400 \mathrm{~K}$ for 30 minutes. $\mathrm{NiAl}(110)$ reciprocal-space surface unit cell is marked in (A) with crystallographic directions.

Fig. 5: LEED patterns at $230 \mathrm{~V}$ following Ag adsorption in the LEED chamber.

Fig. 6: LEED patterns at $250 \mathrm{~V}$ following Ag adsorption in the LEED chamber. The $(-2,2)$ spot of the substrate and the $(-2,2)$ spot of the hexagonal pattern are marked by red circles in (A,H,J and R). The surface unit cell of the hexagonal pattern is also marked in $(\mathrm{R})$. 
Fig. 7: LEED chamber data. (A) shows the reciprocal-space lattice aspect ratio and (B) shows the lattice size difference between the pattern after 3 ML Ag deposition and the clean surface.

Fig. 8: STM chamber data. (A) shows the reciprocal-space lattice aspect ratio and (B) shows the lattice size difference between the pattern after 3 ML Ag deposition and the clean surface.

Fig. 9: Close-up images of the extra spots appear in the diffraction patterns for different Ag coverages. Regions of the diffraction pattern are marked as black boxes. Beam voltage: (A) $210 \mathrm{~V}$; (B) $210 \mathrm{~V}$; (C) $230 \mathrm{~V}$.

Fig. 10: Reproduced with permission from Fig. 9 in Ref. [1]. DFT predictions for the rippled structure of $\mathrm{a} \operatorname{Ag}(110)$ bilayer of periodicities a $n=2$ and $b \mathrm{n}=3$. To make the rippling visible, all deviations of all species, in all directions from the unrippled bilayer $\mathrm{Ag}(110)$ atom positions are magnified by a factor of 10 . Open circles are $\mathrm{Ag}$, darkest circles are $\mathrm{Ni}$, and light gray circles are $\mathrm{Al}$.

Fig. 11: Schematic presentation of overlaying $\mathrm{Ag}(111)$ surface lattice on $\mathrm{NiAl}(110)$ surface lattice. $\mathrm{NiAl}(110)$ and $\mathrm{Ag}(111)$ surface unit cells are marked as black rectangle and red diamond respectively. Blue solid circles represent $\mathrm{Ni}$ atoms, blue rings represent $\mathrm{Al}$ atoms and red rings represent $\mathrm{Ag}$ atoms. 


\section{Figures}
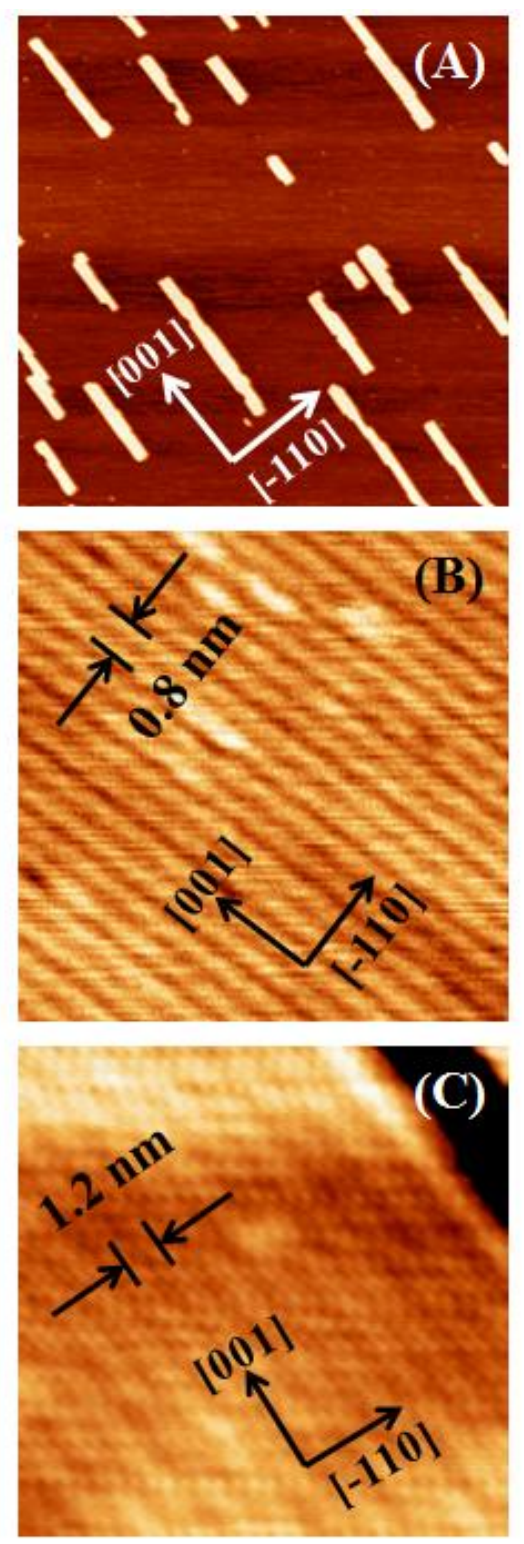

Fig. 1 


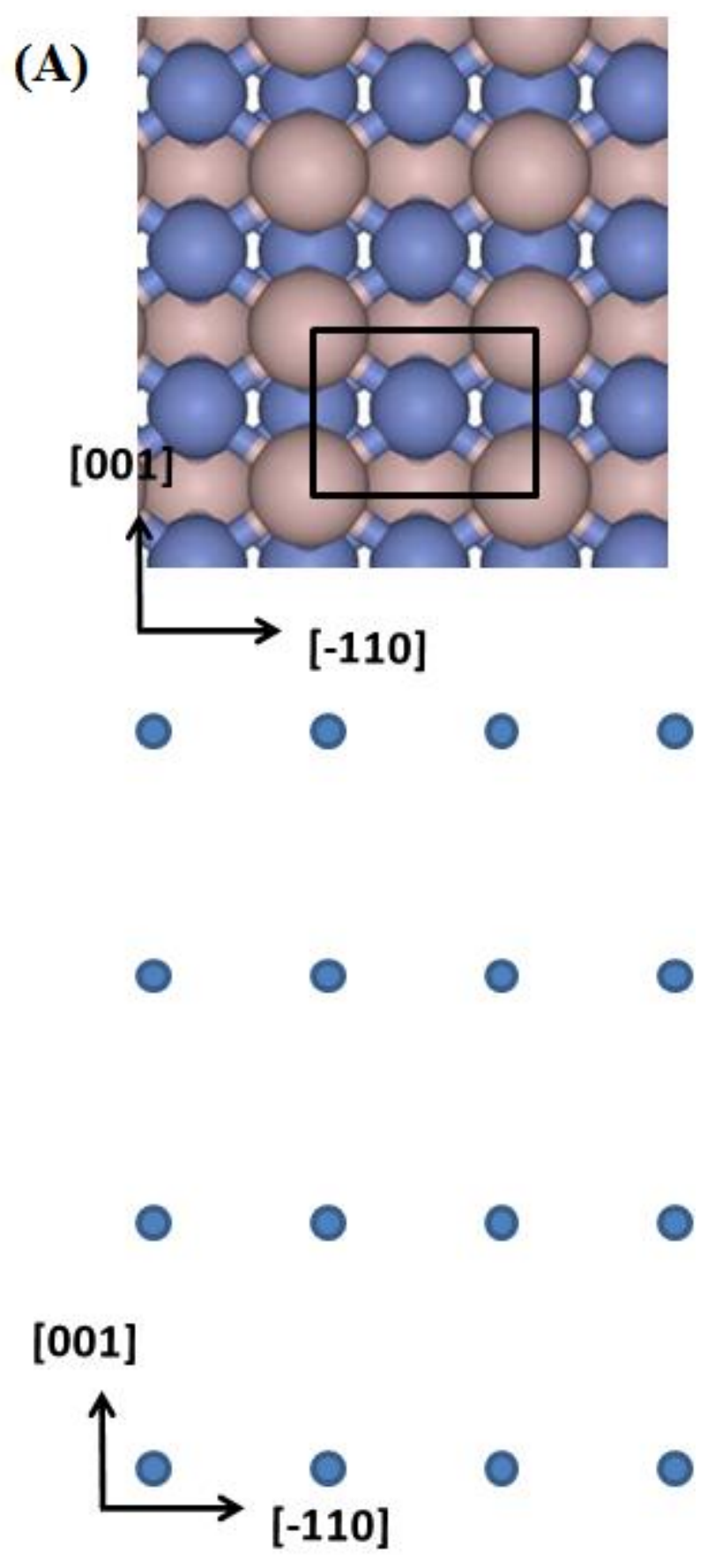

Fig. 2A 

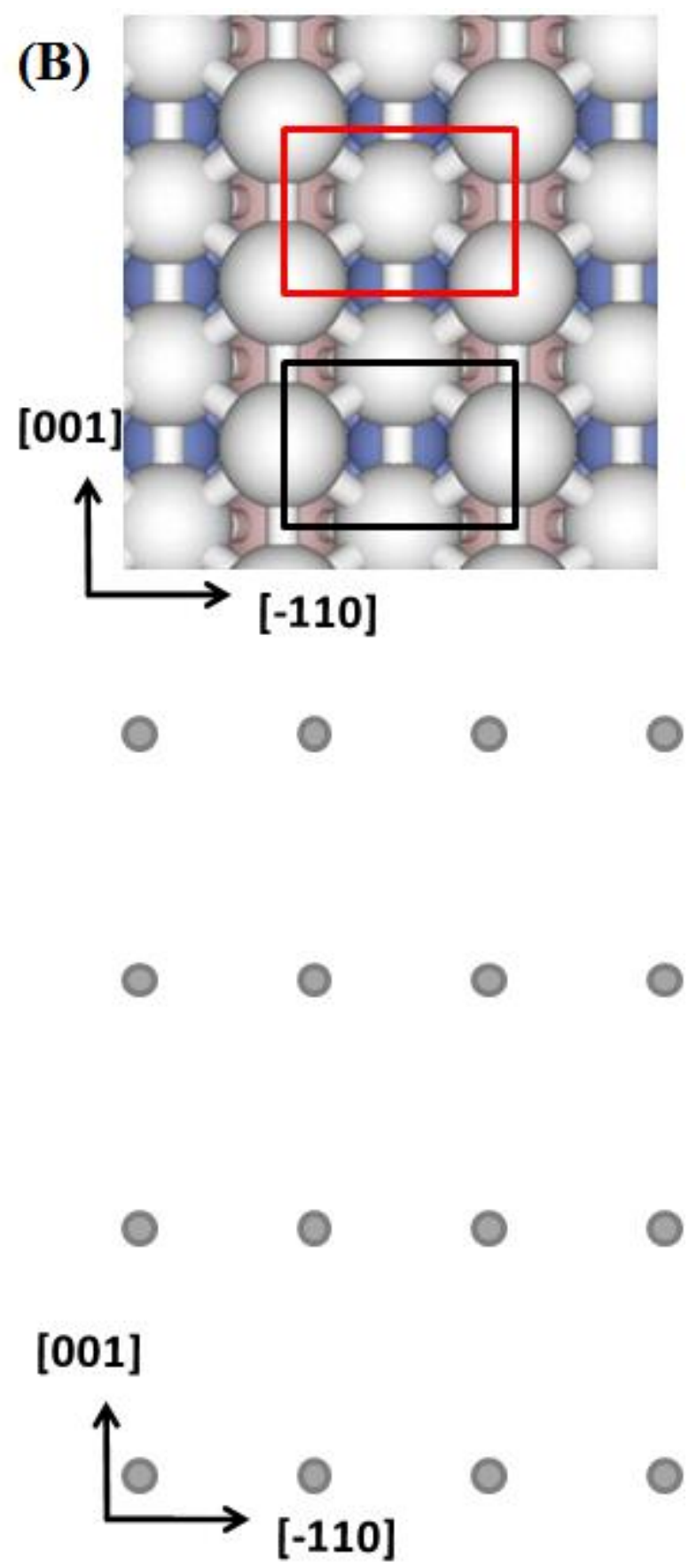

Fig. 2B 


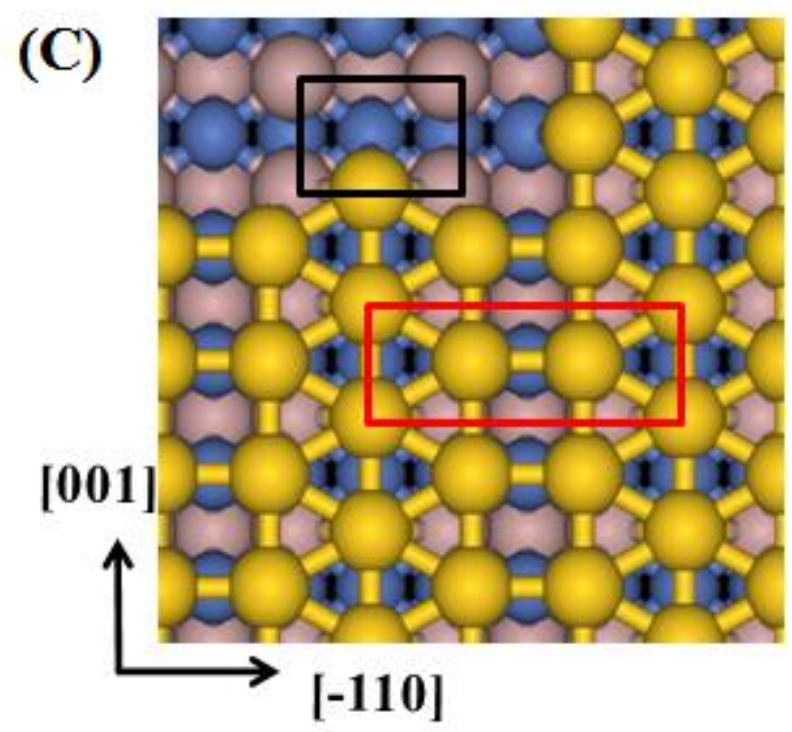

○ $\bigcirc \circ$

- $\bigcirc \circ$

[001]

$\stackrel{\uparrow}{\longrightarrow} \underset{[-110]}{ } \odot$

Fig. 2C 


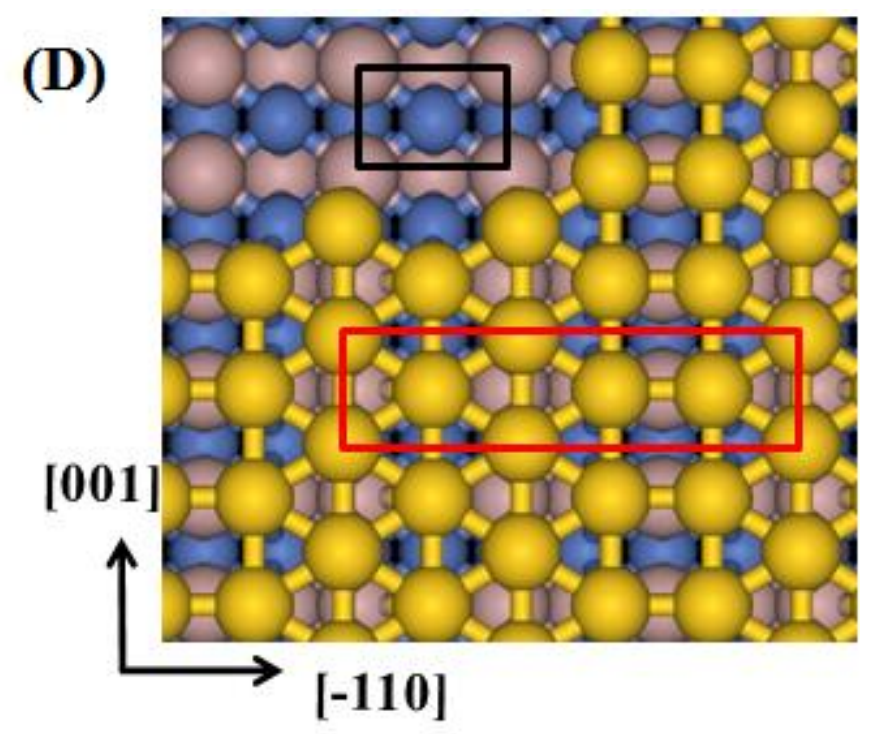

○ - 0 - 0

○ $\bigcirc-\bullet$

[001]

$\stackrel{\ominus}{\longrightarrow} \underset{[-110]}{\odot} \bullet \bullet \odot$

Fig. 2D 


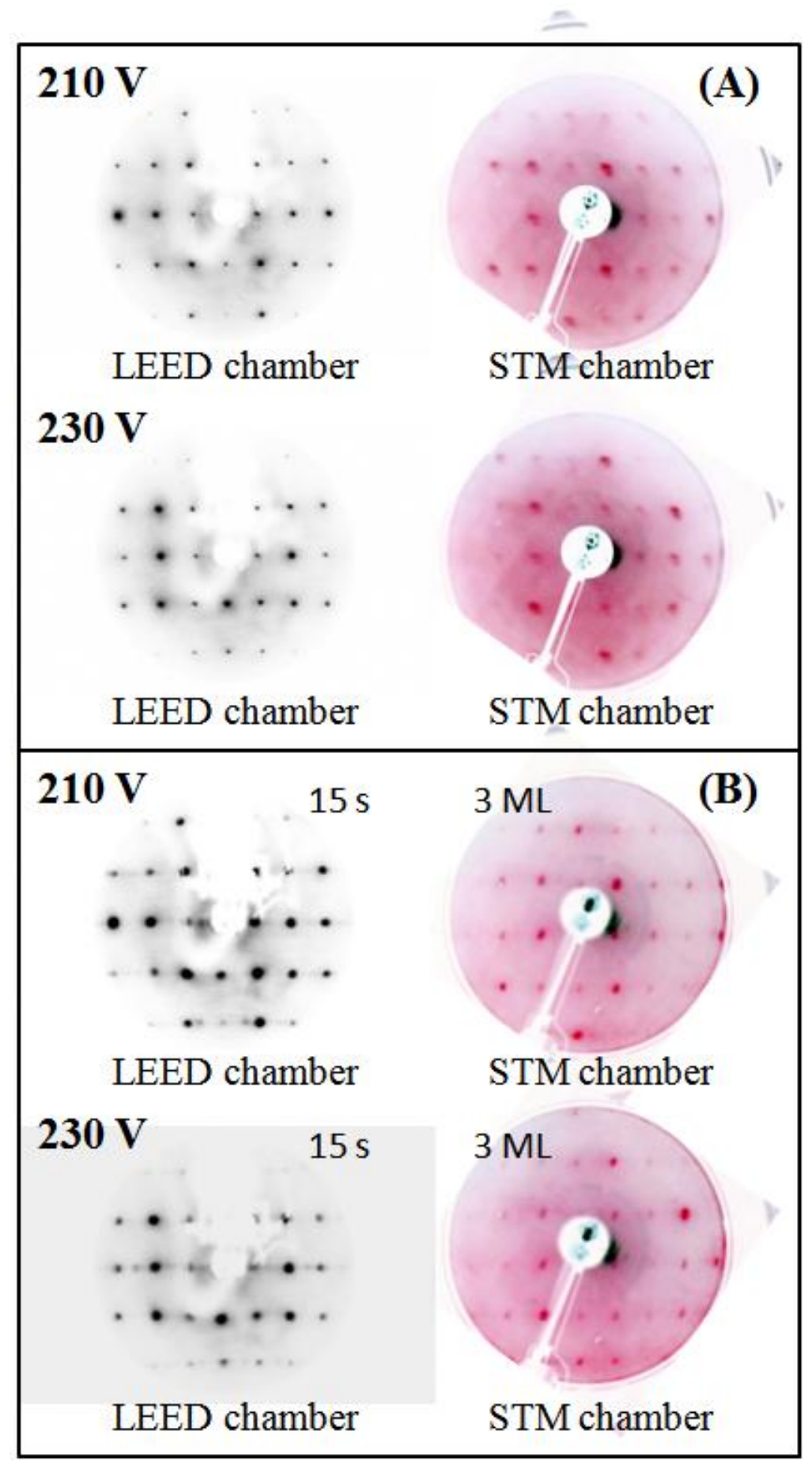

Fig. 3 


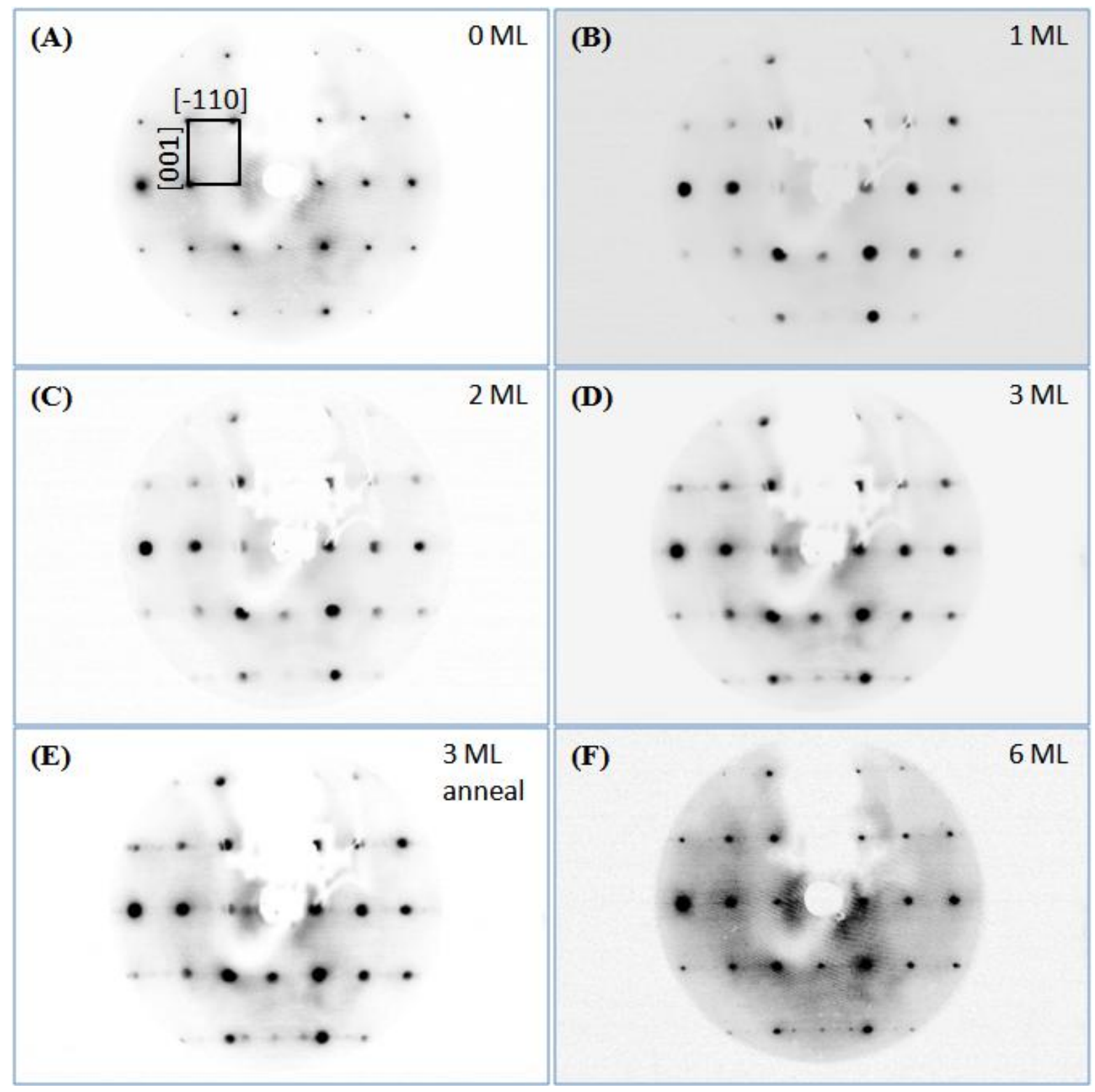

Fig. $4(1 / 3)$ 


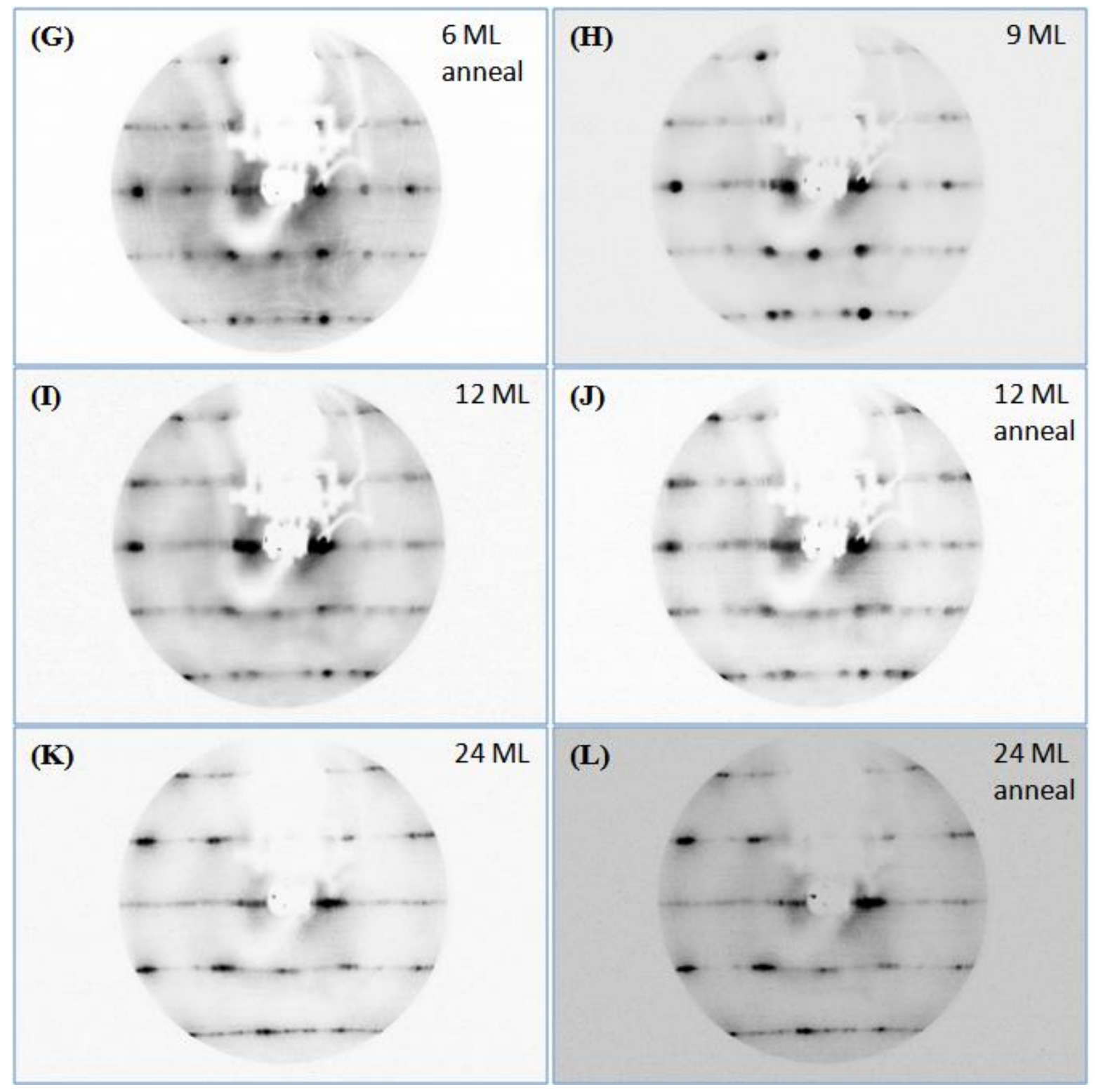

Fig. $4(2 / 3)$ 


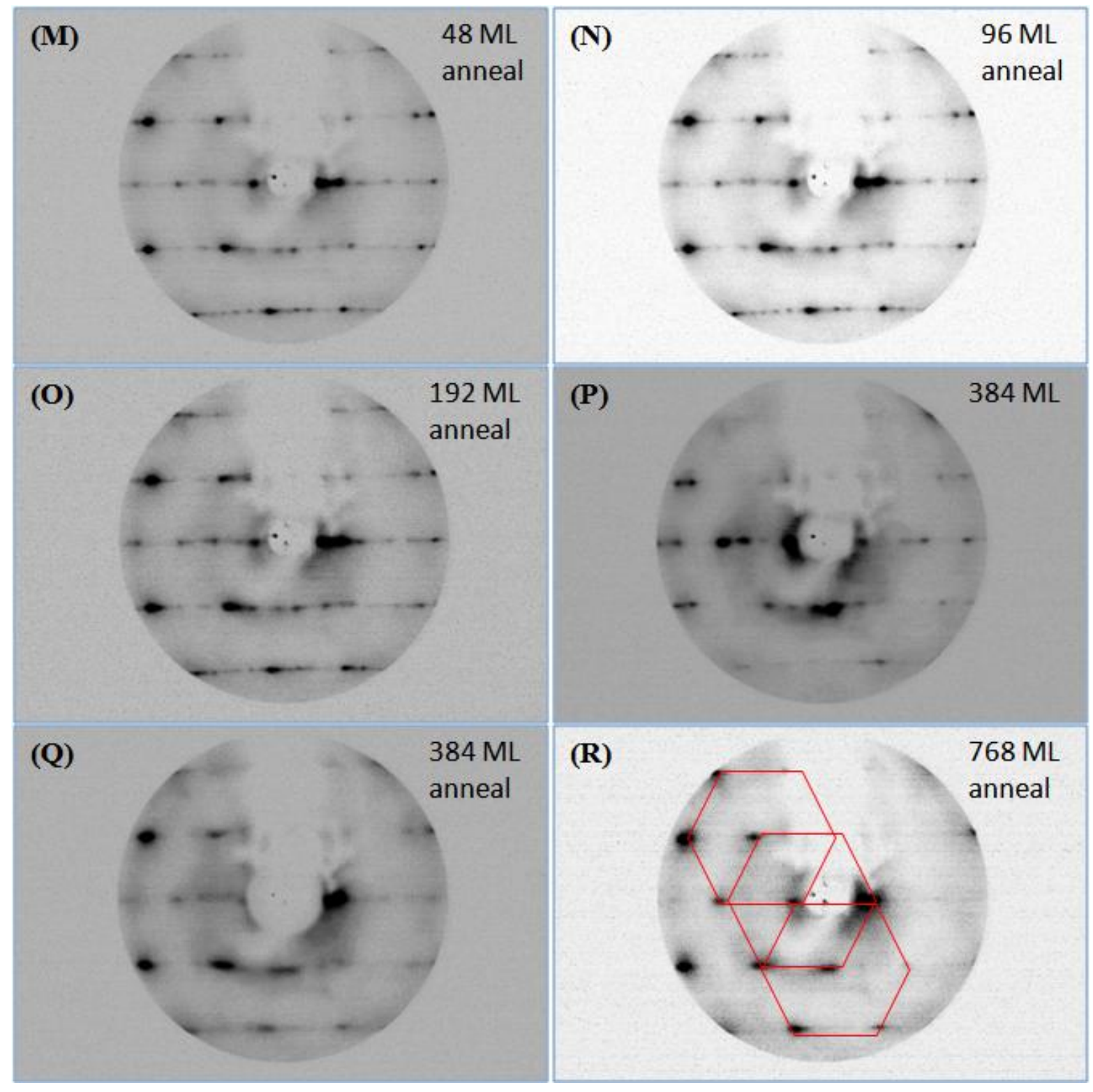

Fig. $4(3 / 3)$ 


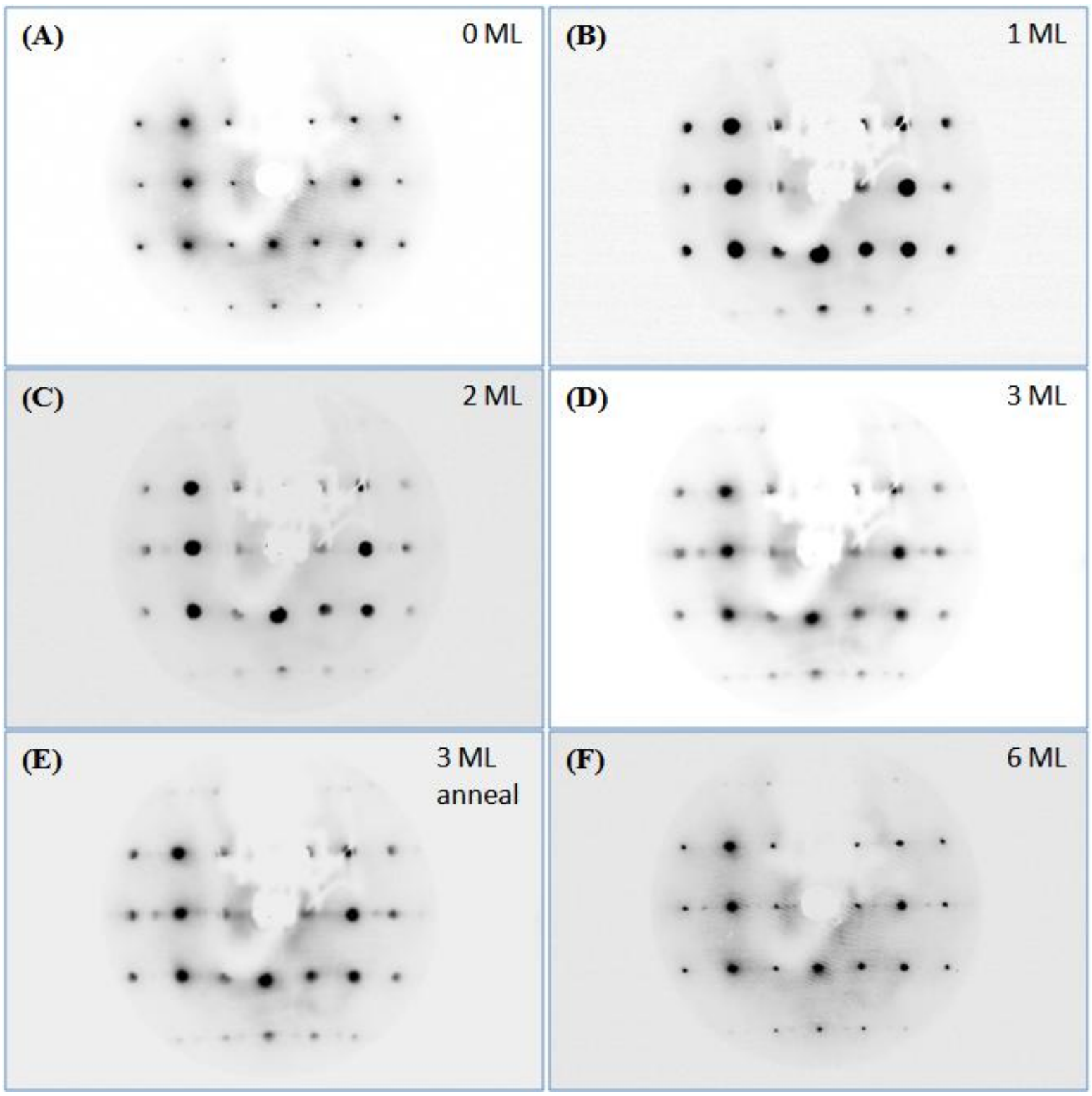

Fig. $5(1 / 3)$ 


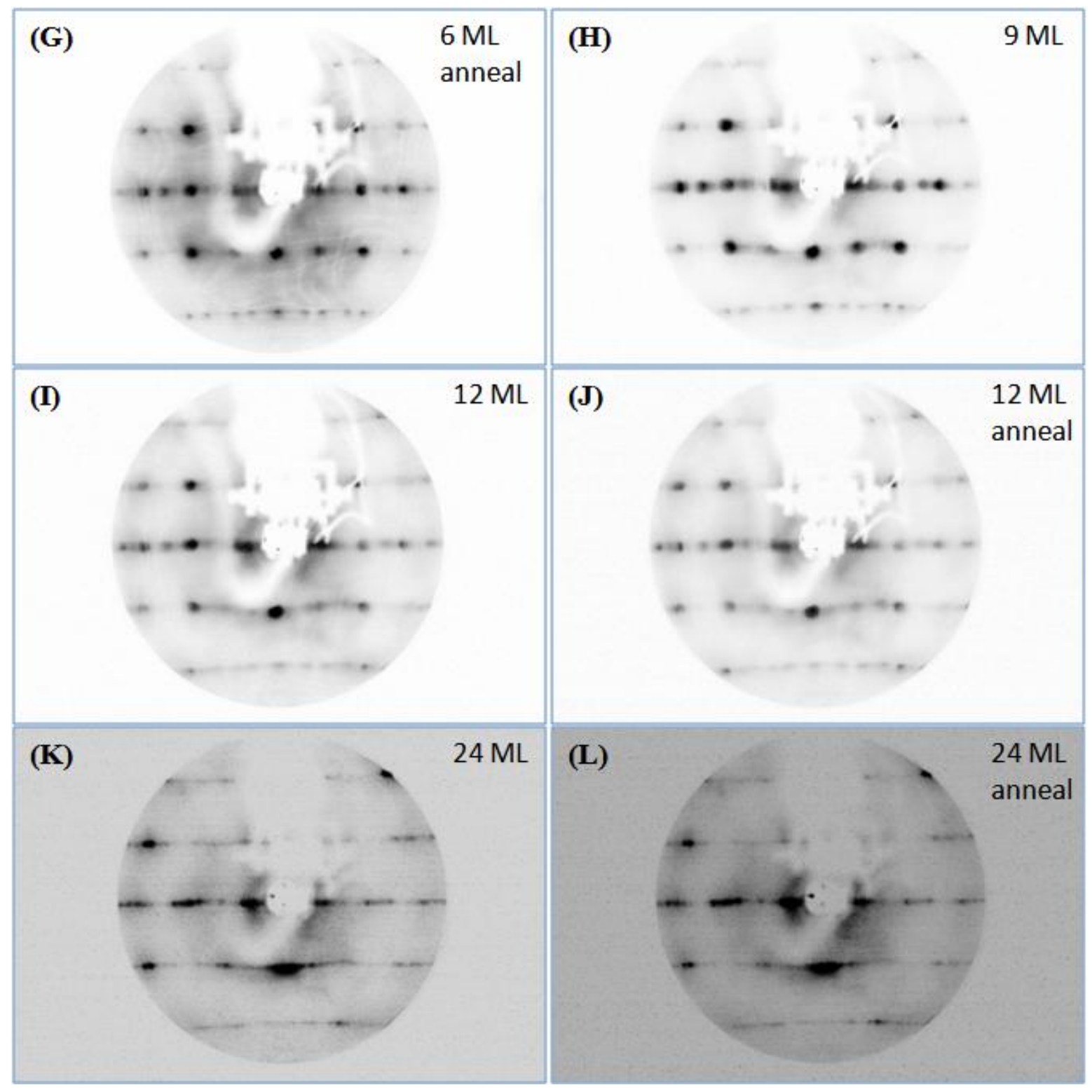

Fig. $5(2 / 3)$ 


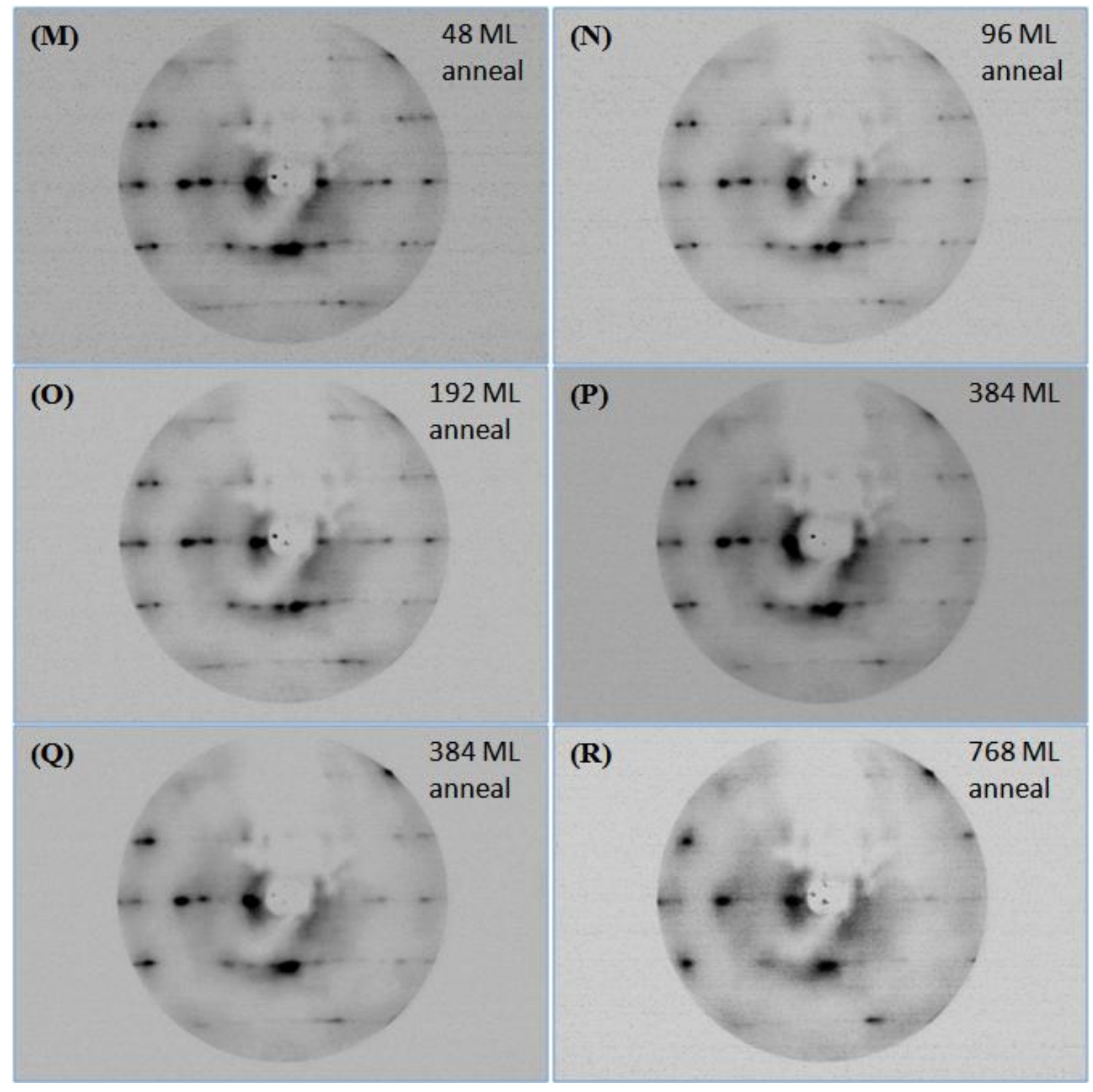

Fig. $5(3 / 3)$ 


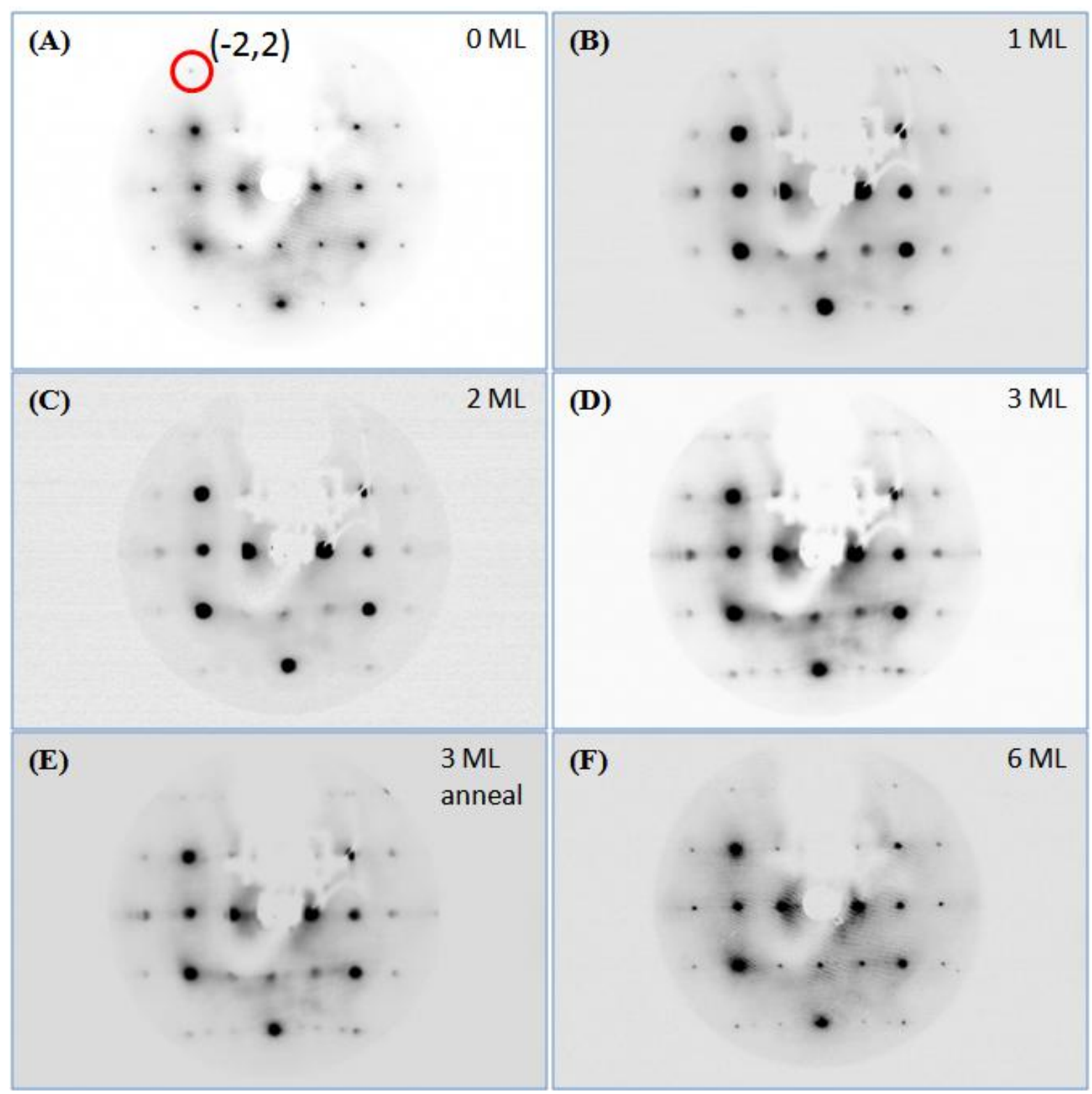

Fig. $6(1 / 3)$ 


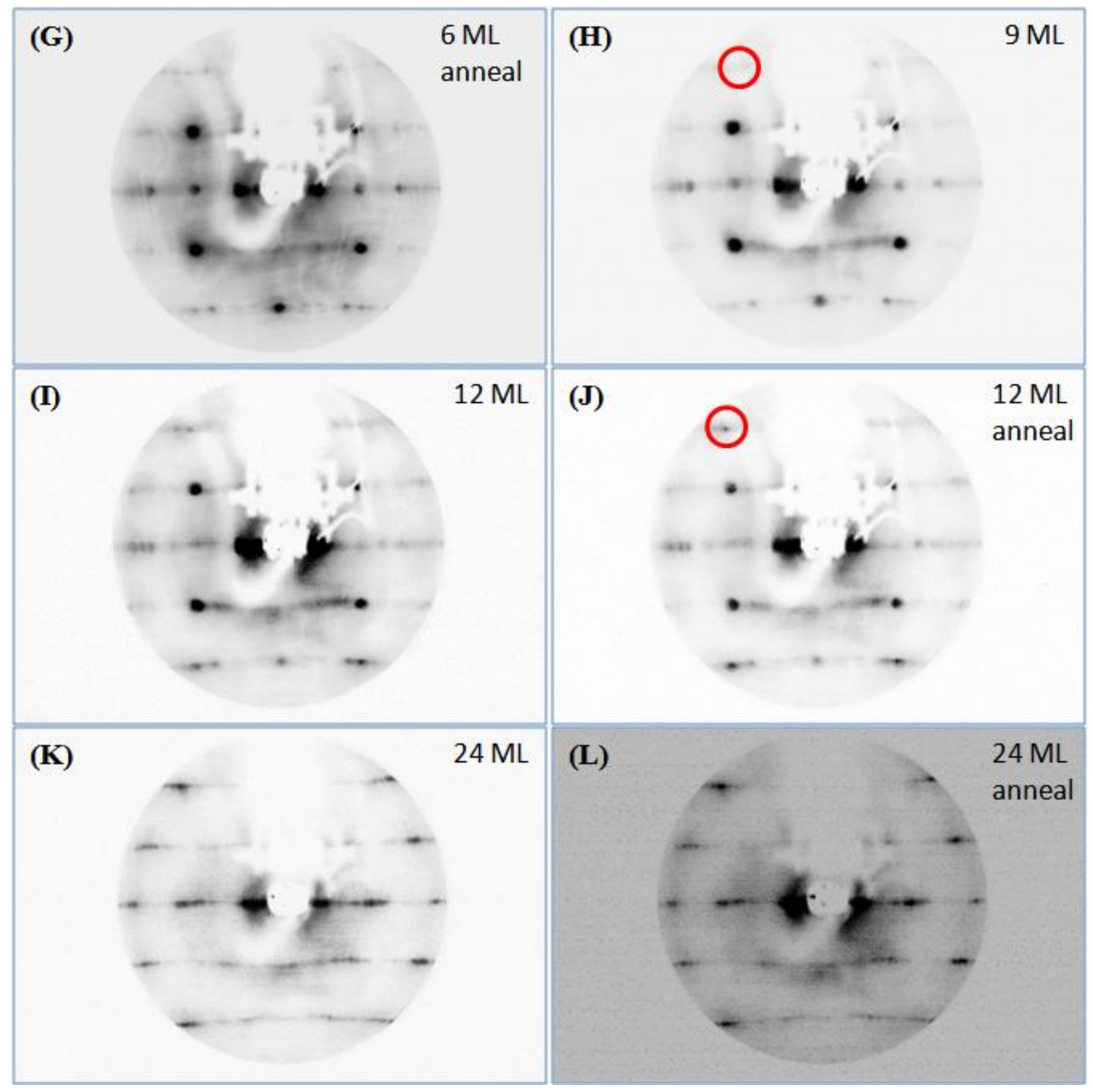

Fig. $6(2 / 3)$ 


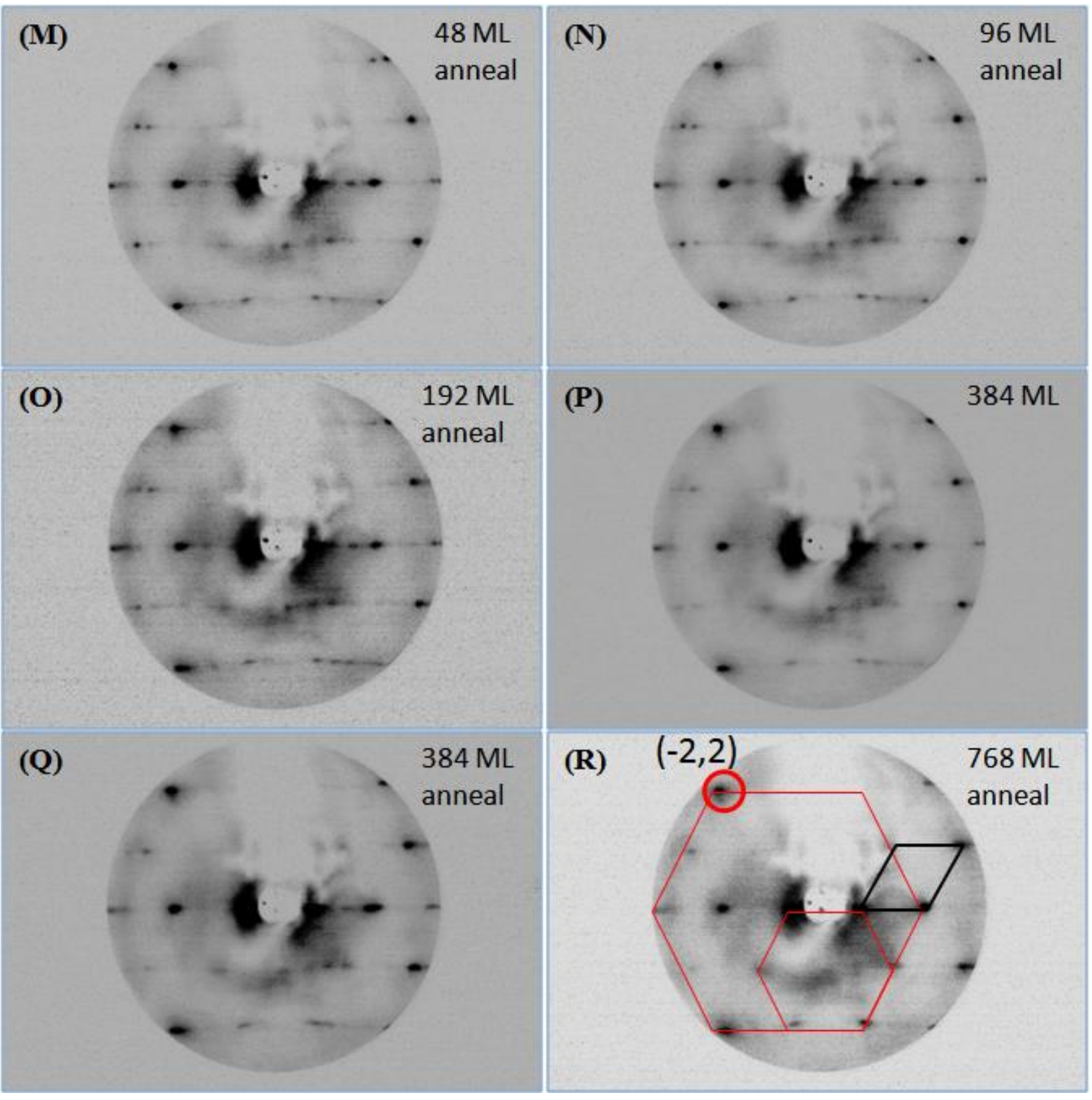

Fig. $6(3 / 3)$ 

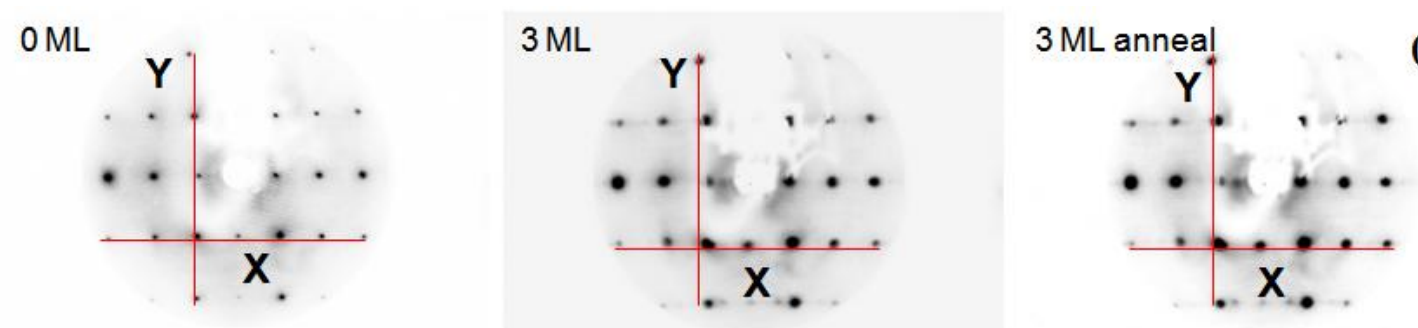

(A)

\begin{tabular}{|c|c|c|c|}
\hline \multirow{2}{*}{$\begin{array}{c}\text { Beam } \\
\text { voltage }\end{array}$} & \multicolumn{3}{|c|}{ Lattice aspect ratio $(\mathrm{Y} / 4: \mathrm{X} / 6)$} \\
\cline { 2 - 4 } & Clean surface & $3 \mathrm{ML} \mathrm{Ag}$ & $3 \mathrm{ML}$ annealed \\
\hline $210 \mathrm{~V}$ & 1.448 & 1.448 & 1.423 \\
\hline $230 \mathrm{~V}$ & 1.433 & 1.420 & 1.414 \\
\hline $250 \mathrm{~V}$ & 1.425 & 1.412 & 1.414 \\
\hline $270 \mathrm{~V}$ & 1.416 & 1.408 & 1.419 \\
\hline $290 \mathrm{~V}$ & 1.410 & 1.398 & 1.414 \\
\hline Average & $\mathbf{1 . 4 2 6 \pm 0 . 0 1 5}$ & $\mathbf{1 . 4 1 7} \pm \mathbf{0 . 0 1 9}$ & $\mathbf{1 . 4 1 7} \pm \mathbf{0 . 0 0 4}$ \\
\hline
\end{tabular}

Fig. 7A 
OML

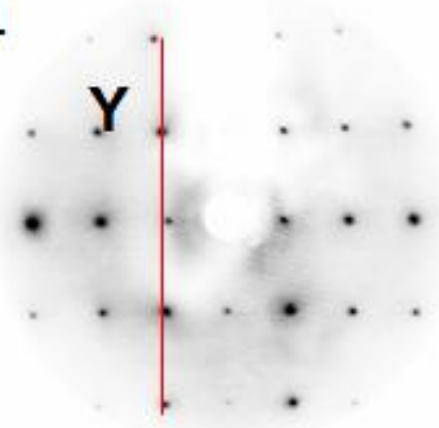

$3 \mathrm{ML}$

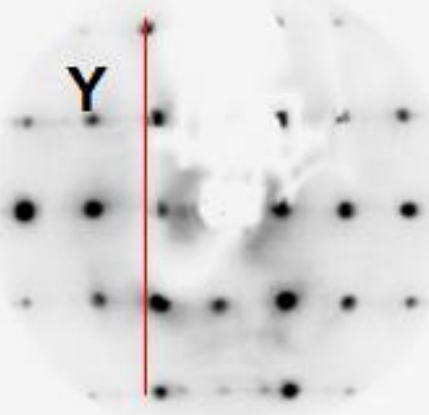

(B)

\begin{tabular}{|c|c|}
\hline $\begin{array}{c}\text { Beam } \\
\text { voltage }\end{array}$ & $\begin{array}{c}\text { Lattice size difference } \\
{[\mathrm{Y}(3 \mathrm{ML})-\mathrm{Y}(\mathrm{OML})] / \mathrm{Y}(3 \mathrm{ML})}\end{array}$ \\
\hline $210 \mathrm{~V}$ & $0.23 \%$ \\
\hline $230 \mathrm{~V}$ & $0.34 \%$ \\
\hline $250 \mathrm{~V}$ & $0.25 \%$ \\
\hline $270 \mathrm{~V}$ & $0.11 \%$ \\
\hline $290 \mathrm{~V}$ & $0.34 \%$ \\
\hline Average & $\mathbf{0 . 2 5} \mathbf{0 . 0 9} \%$ \\
\hline
\end{tabular}

Fig. 7B 


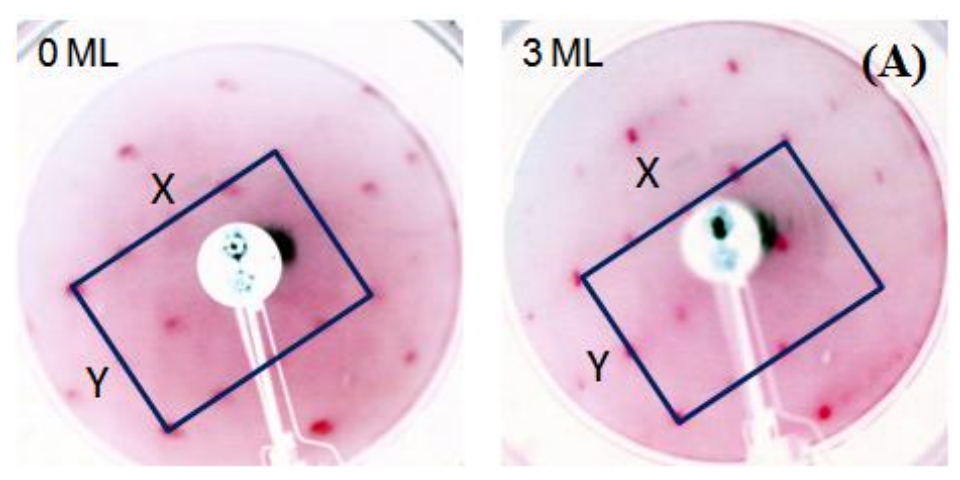

\begin{tabular}{|c|c|c|}
\hline \multirow{2}{*}{$\begin{array}{c}\text { Beam } \\
\text { voltage }\end{array}$} & \multicolumn{2}{|c|}{ Lattice aspect ratio (Y/2 : X/4) } \\
\cline { 2 - 3 } & Clean surface & $3 \mathrm{ML} \mathrm{Ag}$ \\
\hline $100 \mathrm{~V}$ & 1.424 & 1.423 \\
\hline $105 \mathrm{~V}$ & 1.420 & 1.414 \\
\hline $110 \mathrm{~V}$ & 1.416 & 1.416 \\
\hline $115 \mathrm{~V}$ & 1.415 & 1.428 \\
\hline $120 \mathrm{~V}$ & 1.400 & 1.414 \\
\hline $125 \mathrm{~V}$ & 1.423 & 1.415 \\
\hline $130 \mathrm{~V}$ & 1.415 & 1.414 \\
\hline $135 \mathrm{~V}$ & 1.403 & 1.406 \\
\hline $140 \mathrm{~V}$ & 1.419 & 1.410 \\
\hline $145 \mathrm{~V}$ & 1.420 & 1.405 \\
\hline $150 \mathrm{~V}$ & 1.416 & 1.411 \\
\hline $155 \mathrm{~V}$ & 1.416 & 1.413 \\
\hline $160 \mathrm{~V}$ & 1.413 & 1.417 \\
\hline $170 \mathrm{~V}$ & 1.403 & 1.423 \\
\hline $180 \mathrm{~V}$ & 1.421 & 1.414 \\
\hline Average & $1.415 \pm 0.007$ & $1.415 \pm 0.006$ \\
\hline
\end{tabular}

Fig. 8A 


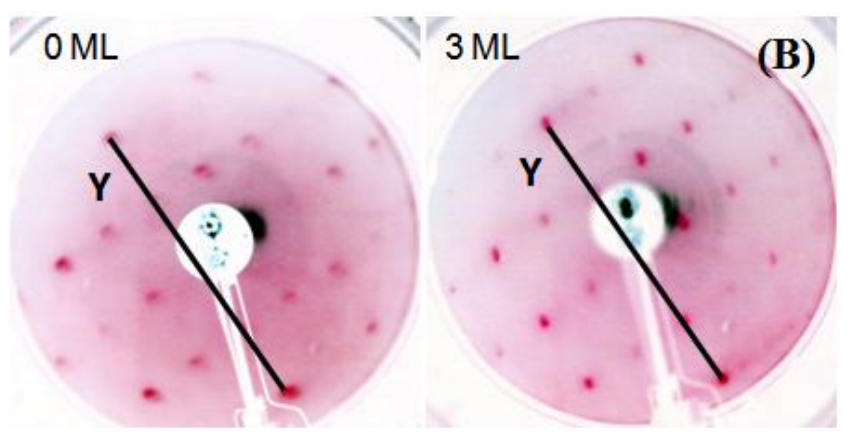

\begin{tabular}{|c|c|}
\hline $\begin{array}{c}\text { Beam } \\
\text { voltage }\end{array}$ & $\begin{array}{c}\text { Lattice size difference } \\
{[\mathrm{Y}(3 \mathrm{ML})-\mathrm{Y}(0 \mathrm{ML})] / \mathrm{Y}(3 \mathrm{ML})}\end{array}$ \\
\hline $100 \mathrm{~V}$ & $0.23 \%$ \\
\hline $105 \mathrm{~V}$ & $0.54 \%$ \\
\hline $110 \mathrm{~V}$ & $0.70 \%$ \\
\hline $115 \mathrm{~V}$ & $0.58 \%$ \\
\hline $120 \mathrm{~V}$ & $0.37 \%$ \\
\hline $125 \mathrm{~V}$ & $0.42 \%$ \\
\hline $130 \mathrm{~V}$ & $0.17 \%$ \\
\hline $135 \mathrm{~V}$ & $0.43 \%$ \\
\hline $140 \mathrm{~V}$ & $-0.53 \%$ \\
\hline $145 \mathrm{~V}$ & $-0.11 \%$ \\
\hline $150 \mathrm{~V}$ & $0.47 \%$ \\
\hline $155 \mathrm{~V}$ & $0.48 \%$ \\
\hline $160 \mathrm{~V}$ & $0.60 \%$ \\
\hline $165 \mathrm{~V}$ & $0.59 \%$ \\
\hline $170 \mathrm{~V}$ & $0.41 \%$ \\
\hline $175 \mathrm{~V}$ & $0.43 \%$ \\
\hline $180 \mathrm{~V}$ & $0.43 \%$ \\
\hline $185 \mathrm{~V}$ & $0.31 \%$ \\
\hline $190 \mathrm{~V}$ & $0.31 \%$ \\
\hline $195 \mathrm{~V}$ & $0.42 \%$ \\
\hline $200 \mathrm{~V}$ & $0.36 \%$ \\
\hline Average & $\mathbf{0 . 3 6} \mathbf{0 . 1 3 \%}$ \\
\hline & \\
\hline & \\
\hline & \\
\hline 190
\end{tabular}

Fig. 8B 

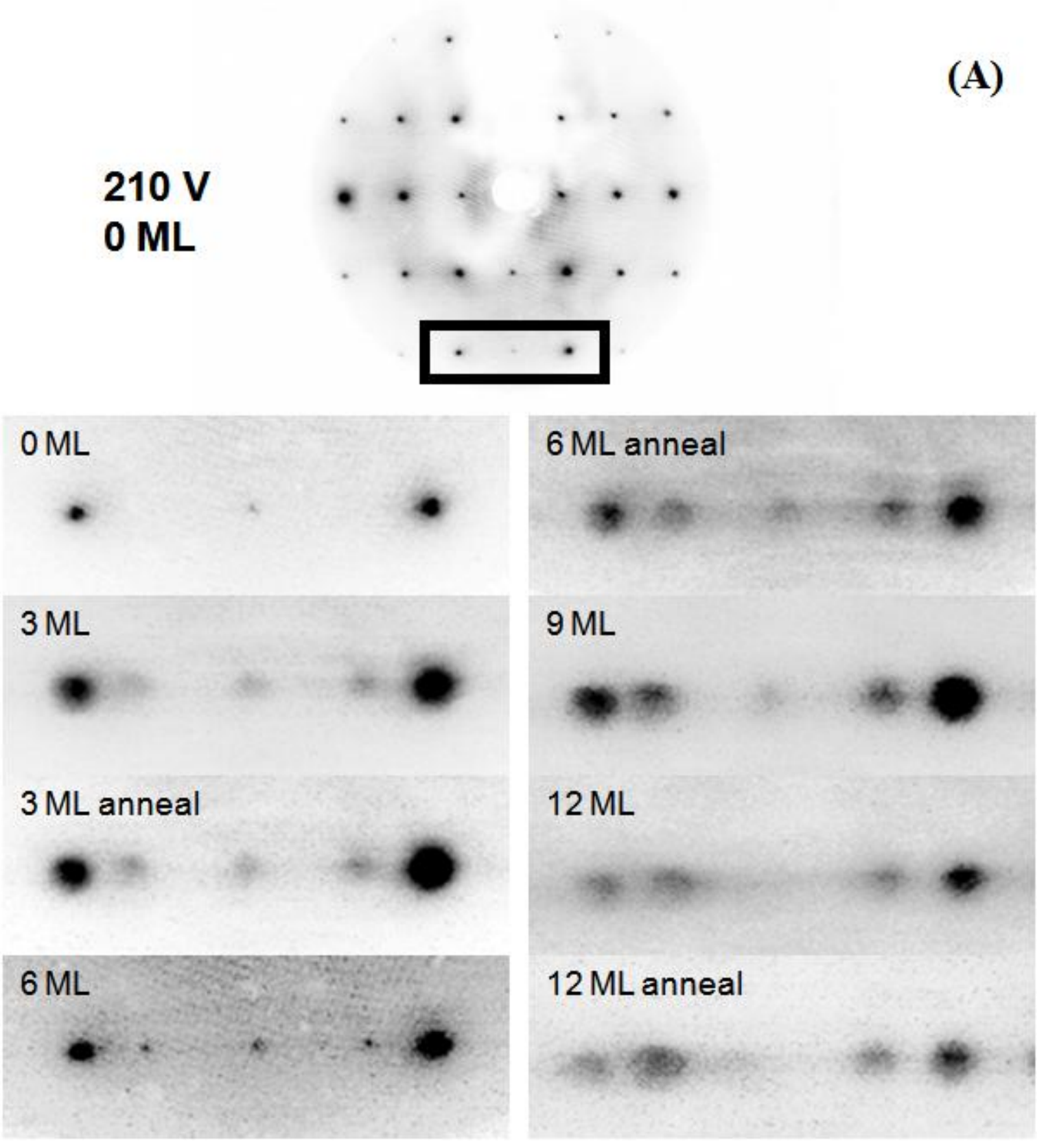

Fig. 9A 
$210 \mathrm{~V}$
$12 \mathrm{ML}$

(B)

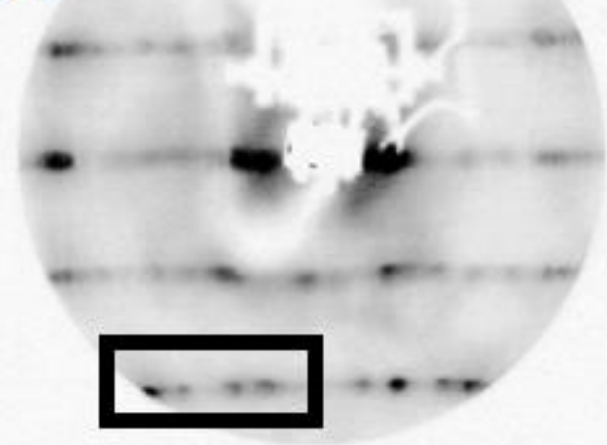

$12 \mathrm{ML}$

$12 \mathrm{ML}$ anneal

Fig. 9B 
(C)
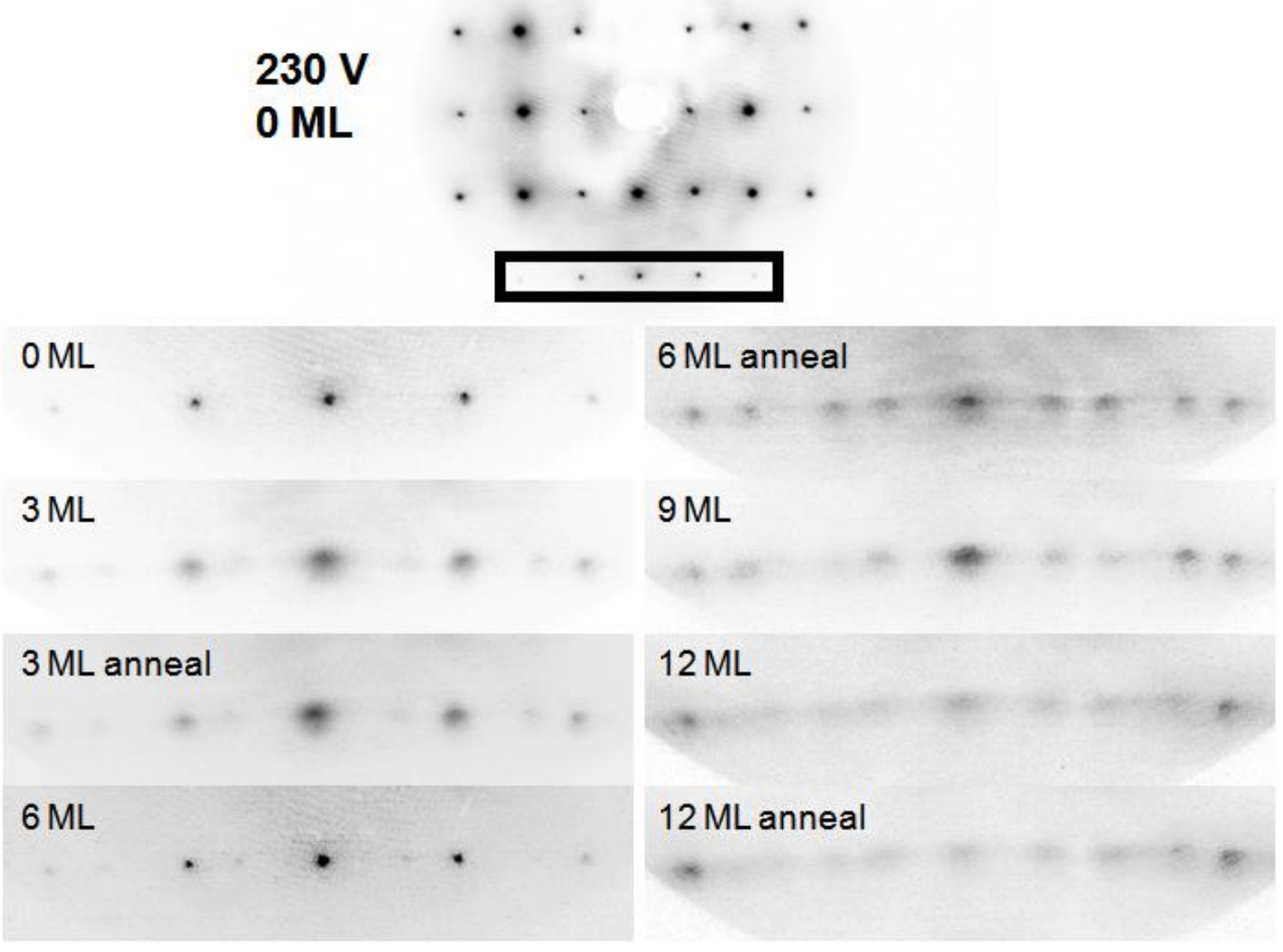

Fig. 9C 

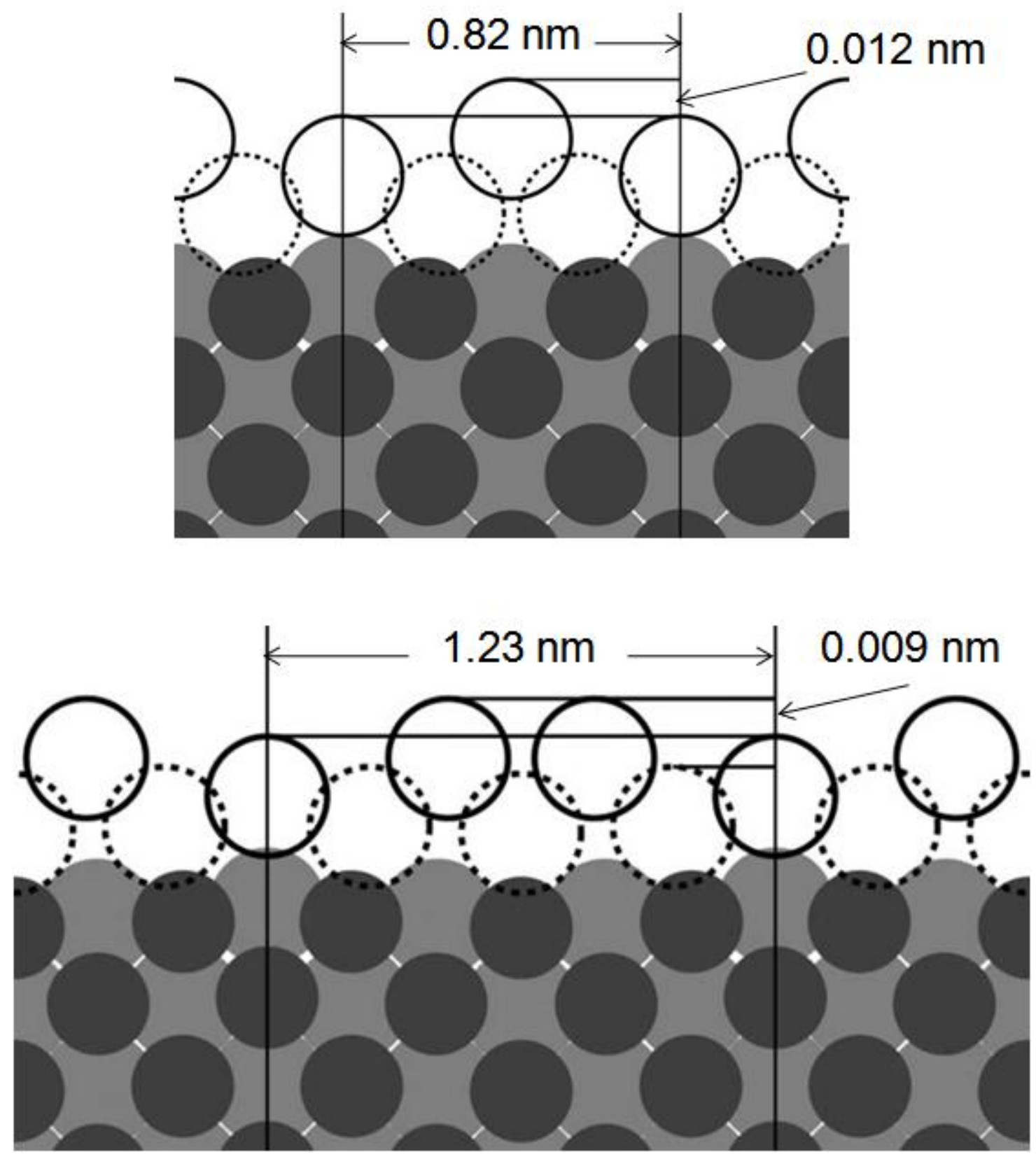

Fig. 10 
$0 \quad 0 \quad 0$
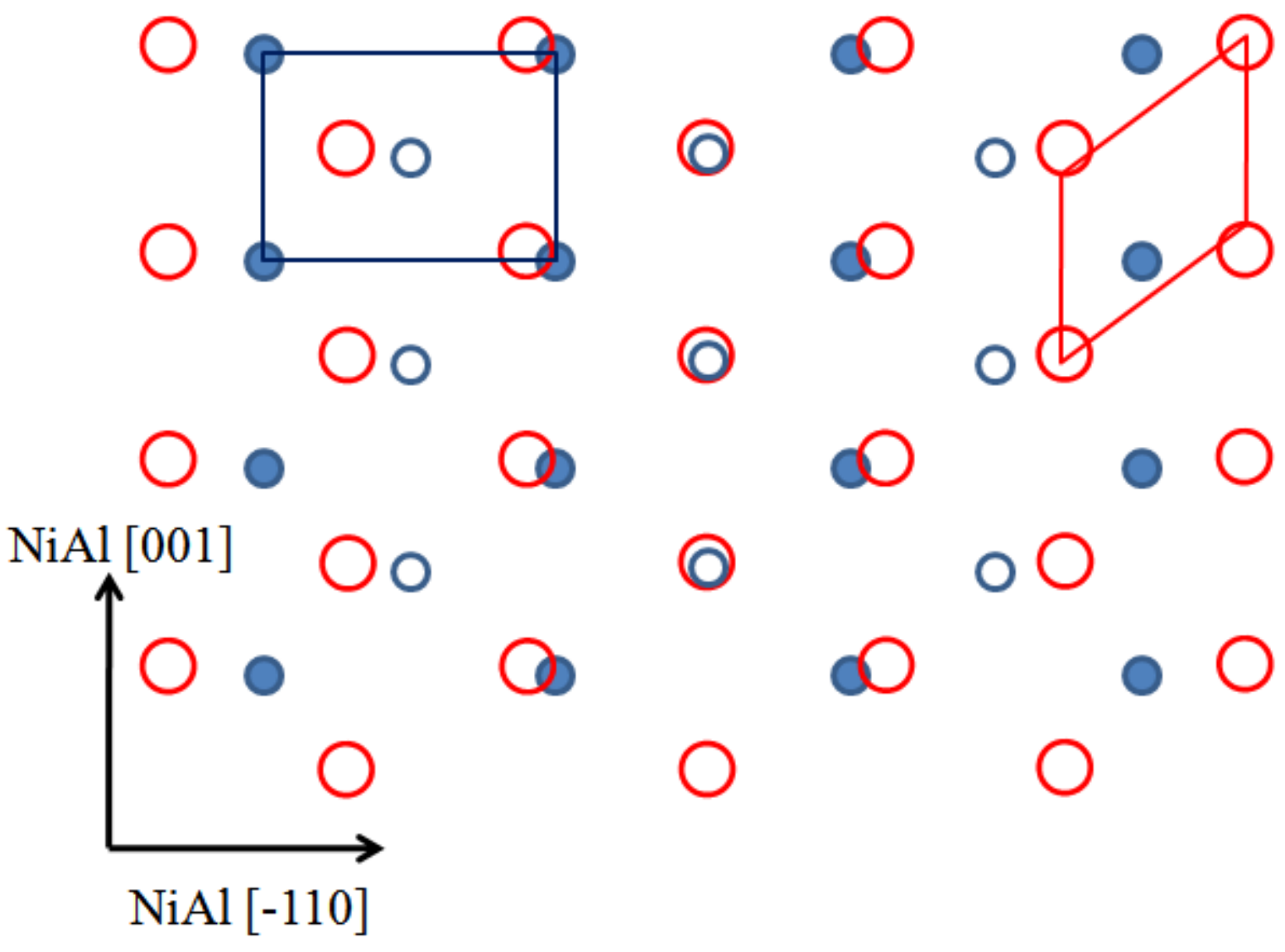

Fig. 11 


\section{APPENDIX: SUPPLEMENTAL EXPERIMENTAL DETAILS FOR CHAPTER 4}

\section{Homemade e-beam evaporator}

The Ag source in the LEED chamber is a homemade e-beam evaporator, originally designed and built by Alex Belianinov, another graduate student in the group. Fig. 1 shows a schematic diagram of the evaporator head and its principle of operation. This evaporator is equipped with two Ta crucibles, with a cap on each crucible. Each cap has a $1.25 \mathrm{~mm}$ orifice at the center. This makes the source more like a Knudsen cell. One can put different target metals in the two crucibles to do co-deposition either simultaneously or sequentially. The crucibles are isolated by a water-cooled shroud to minimize contamination. Due to space limitations, the $\mathrm{W}$ filaments are placed on top of the crucibles instead of underneath them. Since there is perfect line-of-sight for the electron beam, no electron focusing mechanism is used. To start and stop deposition, a movable shutter located near the source is used.

Fig. 1(B) is a schematic diagram showing the principle of operation for a single crucible. The crucible is positively biased. The bias voltage can be tuned up to $3000 \mathrm{~V}$. Target heating is achieved by passing current through the filament using a $20 \mathrm{~V}, 10 \mathrm{~A}$ DC power supply. Electrons are emitted from the filament and are accelerated towards the crucible. The temperature of the crucible depends on the filament emission current and the bias voltage. The filament emission current is measured by monitoring a potential drop through a $200 \Omega$ resistor. The sample is grounded through a picoammeter, which also shows a fraction of the generated flux current. 


\section{W filament temperature and vapor pressure analysis}

The electron beam used to heat the crucible in our homemade evaporator is generated by thermionic emission from the $\mathrm{W}$ filament. By passing current, the $\mathrm{W}$ filament is heated. As the temperature increases, the probability for the electrons to overcome the surface energy barrier and appear as thermally emitted electrons also increases. However, one needs to realize that as the filament temperature increases, its own vapor pressure also increases. In our design, the filament is not shielded. If the $\mathrm{W}$ vapor pressure increases to a significant value, it may lead to film contamination during deposition. Therefore, it is necessary to investigate the filament temperature and its corresponding vapor pressure.

The temperature $\mathrm{T}$ and the density of the thermionic current $\mathrm{j}$ from a homogeneous metal surface can be related by the Richardson-Dushman equation:[1]

$$
j=A T^{2} \exp \left(-\Phi / k_{B} T\right)
$$

where

$$
A=\frac{4 \pi m k_{B}^{2} e}{h^{3}} \approx 120 \mathrm{Acm}^{-2} \mathrm{~K}^{-2}
$$

with $\mathrm{m}$ and e being the electron mass and charge respectively, $\mathrm{h}$ is Planck's constant, $\mathrm{k}_{\mathrm{B}}$ is the Boltzmann constant and $\Phi$ is the $\mathrm{W}$ work function, which is $4.5 \mathrm{eV}$ taken from Solid State Physics by N. W. Ashcroft and N. D. Mermin.[2]

The filament emission current used in this project is $16 \mathrm{~mA}$. The filament is a $\mathrm{W}$ wire that is $2 \mathrm{~cm}$ long. The diameter is $0.2 \mathrm{~mm}$. Given the emission current and dimensions of the filament, one can calculate the current density $\mathrm{j}$ and therefore estimate the filament temperature $\mathrm{T}$ using the Richardson-Dushman equation. One finds the temperature is $2340 \mathrm{~K}$. At this temperature, the equilibrium $\mathrm{W}$ vapor pressure is found to be $\sim 2 \times 10^{-8}$ Torr.[3] This 
raises the question of whether the $\mathrm{W}$ vapor pressure is high enough to cause contamination during deposition. To answer this question, additional Auger electron spectroscopy (AES) and X-ray photoelectron spectroscopy (XPS) experiments are performed to check the presence of $\mathrm{W}$ on the sample. The results are given in the following section.

\section{Supplemental AES and XPS data}

To check whether there is $\mathrm{W}$ contamination during Ag deposition, additional AES and XPS experiments are performed.[4] First, a thick film of about $800 \mathrm{ML} \mathrm{Ag}$ is deposited on the surface, from the homemade evaporator in the LEED chamber. Next, the $\mathrm{NiAl}(110)$ sample is transferred to a separate UHV chamber equipped with scanning Auger electron spectroscopy (AES). The AES spectrum for the as-loaded sample is shown in Fig. 2. The peaks on the left correspond to Ag MNN transitions. No W signal is detected. In AES, the detection limit is typically quoted as $0.01 \mathrm{ML}[5,6]$ or, in more conventional terms, about $10^{4} \mathrm{ppm}$ in atomic $\%$.

While AES result indicates there is no detectable $\mathrm{W}$ on the surface of the Ag film, it is desirable to also check with other methods and to improve the detection limit, if possible. To this end, we transfer the sample to a third UHV chamber, equipped with X-ray photoelectron spectroscopy (XPS). The XPS results are shown in Fig. 3. The as-loaded sample (Fig. 3A) shows a strong Ag signal and a weak C signal, but no W. This is consistent with the AES result. The sample is then heated to $973 \mathrm{~K}$. If the temperature is high enough, the Ag film will evaporate into vacuum, exposing the substrate surface. Because the vapor pressure of $\mathrm{W}$ at any given temperature is much lower than that of $\mathrm{Ag}$, any $\mathrm{W}$ will be left behind by this treatment and will be more concentrated on the surface by a factor of about 
$10^{3}$. Hence, the purpose of this treatment is to concentrate the $\mathrm{W}$. The result will be to improve the detection limit for $\mathrm{W}$ within the $\mathrm{Ag}$ film by about a factor of $10^{3}$, to about 1 atom in $10^{5}(10 \mathrm{ppm})$.

As shown in Fig. 3B and 3C, when the sample is heated to $573 \mathrm{~K}$, the C 1s peak disappears, meaning the carbon species that adsorbed on the surface during sample transfer desorb when the sample is heated. After the sample is heated to $973 \mathrm{~K}$, the Ag peaks are gone, which shows that the Ag film has completely evaporated. The substrate material is exposed (Ni and $\mathrm{Al}$ peaks show up in Fig. 3C). No W signal shows up in Fig. 3C. This means that there was no $\mathrm{W}$ in the original Ag film, at least not above a level of about $10 \mathrm{ppm}$.

From the AES and XPS experiments, we can draw the conclusion that the $\mathrm{W}$ filament of the e-beam evaporator does not cause significant film contamination when working at the conditions used in our Ag deposition experiments.

\section{Reference}

[1] K. Oura, V.G. Lifshits, A.A. Saranin, A.V. Zotov, M. Katayama, Surface Science, Springer, Berlin, 2003.

[2] N.W. Ashcroft, N.D. Mermin, Solid State Physics, Thomson Brooks/Cole, New York, 1976.

[3] The vapor pressure of metals diagrams are prepared by Richard E. Honig of Radio Corporation of America.

[4] These experiments were performed by J.W. Anderegg of Ames Laboratory, USDOE.

[5] J. Cazaux, Surf Sci 140/1 (1984) 85.

[6] B.A. Polonskii, O.D. Protopopov, Izv. Akad. Nauk SSSR, Ser. Fiz. 50/3 (1986) 429. 


\section{Figure captions}

Fig. 1: (A) Schematic diagram of homemade e-beam evaporator head. (a) W filaments; (b) shutter; (c) Ta crucibles with caps; (d) water-cooled envelop. (B) Principle of operation. (e) sample; (f) 5 V DC power supply; (g) voltmeter; (h) $200 \Omega$ resistor; (i) 2000 V DC power supply; (j) picoammeter.

Fig. 2: As-loaded sample AES data taken at $300 \mathrm{~K}$. The peaks shown in the spectrum are Ag MNN peaks. W MNN peak area is marked by the black box. Inset: W MNN peak region at expanded scale.

Fig. 3: XPS data (A) as-loaded sample at 300 K. (B) heated to 573 K. (C) heated to 973 K. W 4d peak region is marked by the arrow. Inset: $\mathrm{W} 4 \mathrm{~d}$ peak region at expanded scale for $(\mathrm{A}-\mathrm{C})$. 
Figures

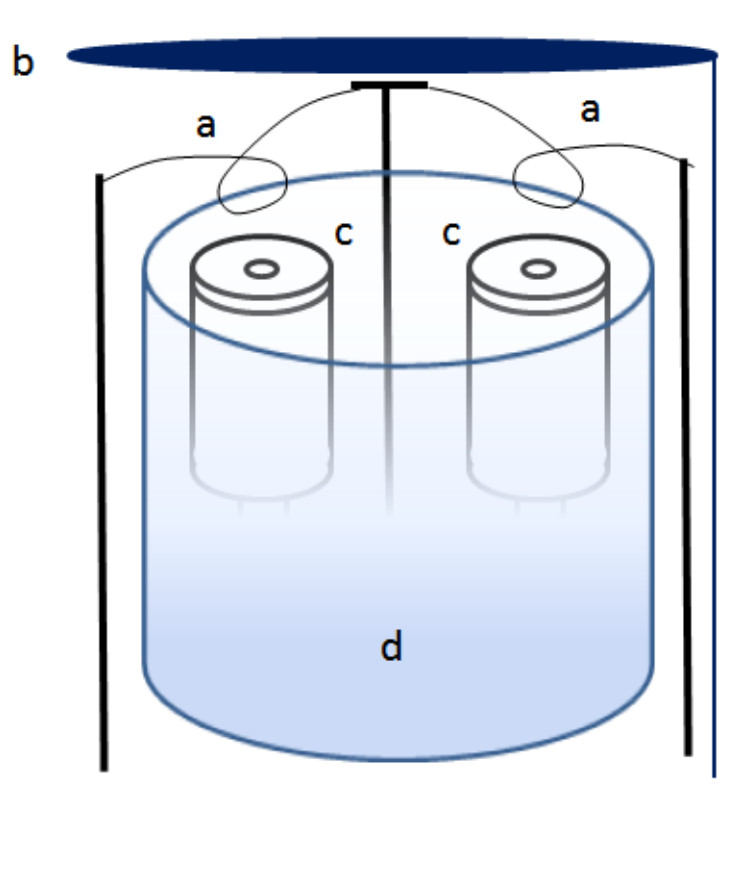

(A)

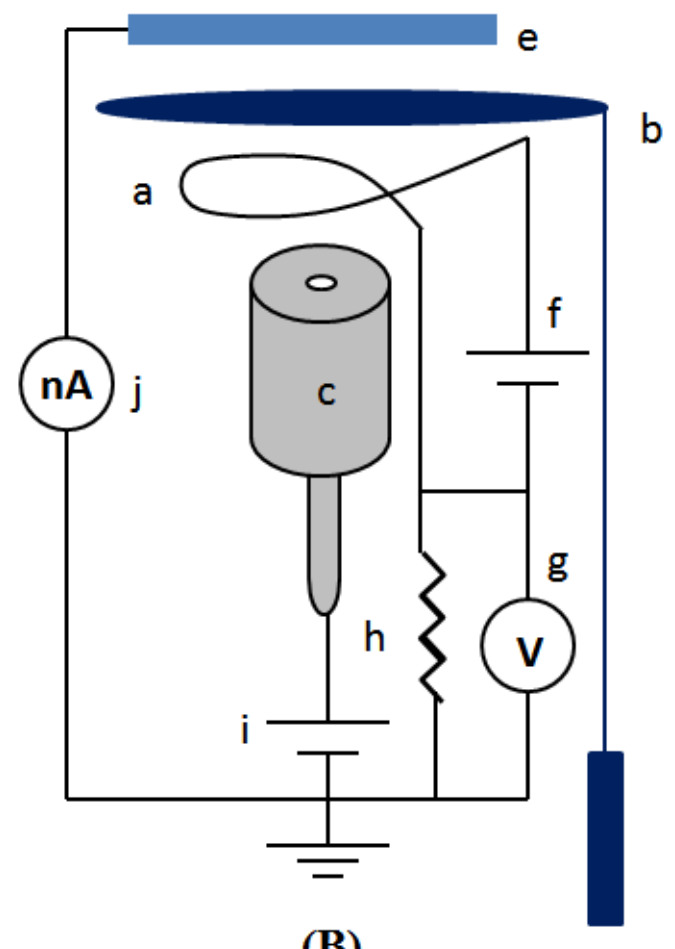

(B)

Fig. 1 
112

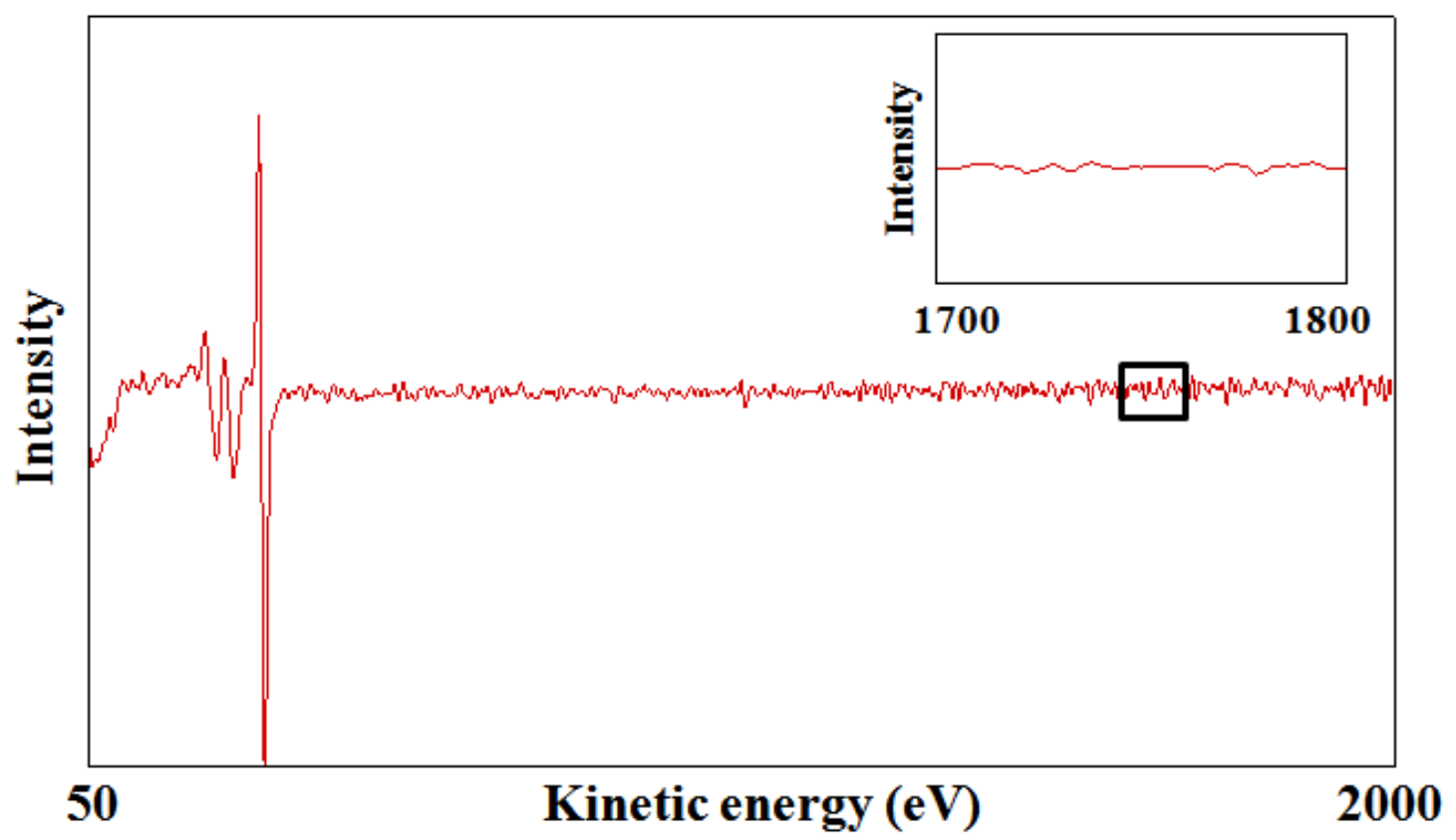

Fig. 2 


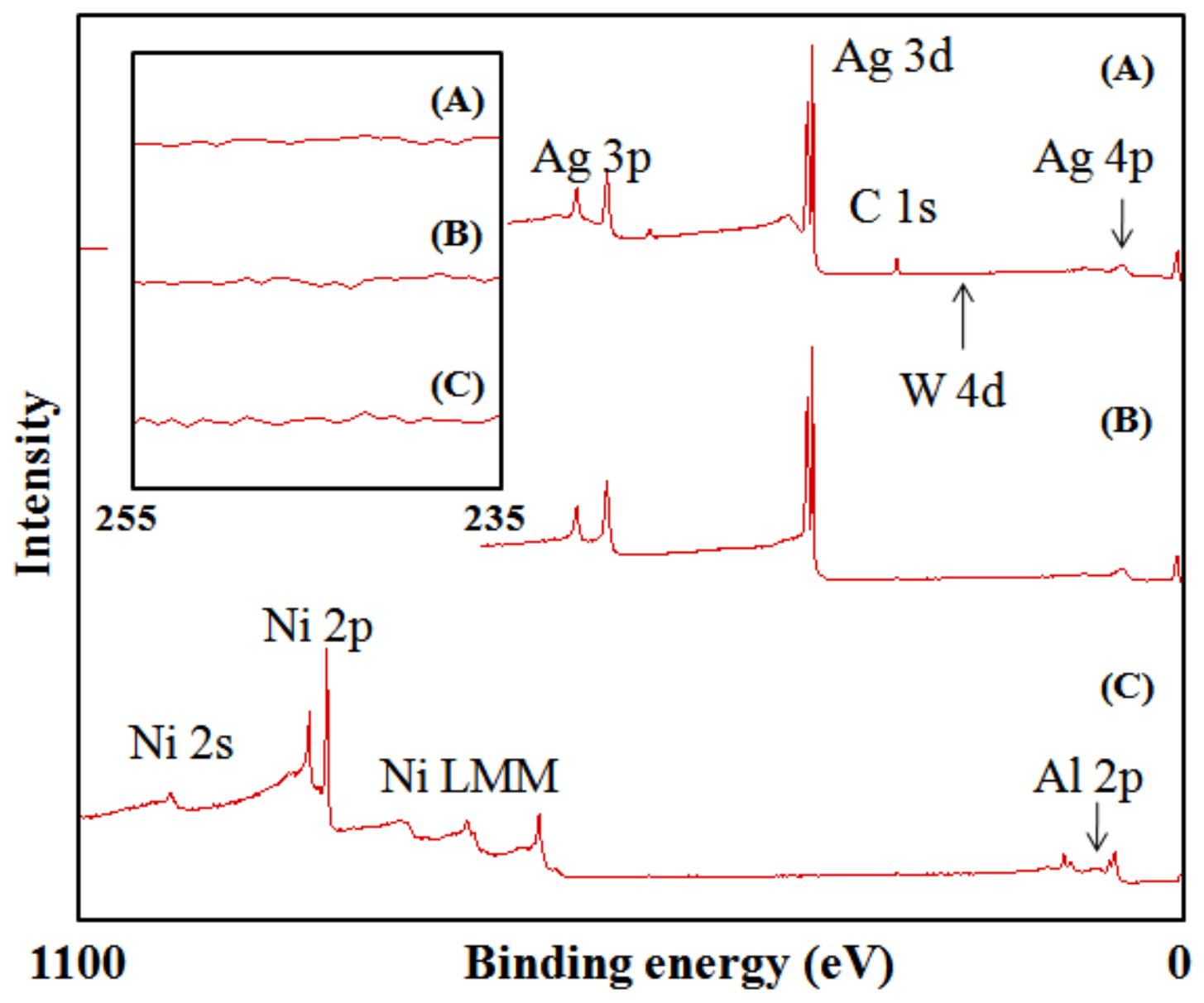

Fig. 3 


\title{
CHAPTER 5. EPITAXIAL GROWTH OF Ni ON NiAI(110) SURFACE
}

\begin{abstract}
Scanning tunneling microscopy (STM) studies of the deposition of Ni on bcc$\mathrm{NiAl}(110)$ were performed in the temperature range from $200 \mathrm{~K}$ to $400 \mathrm{~K}$ mostly in the submonolayer regime. Submonolayer coverages of Ni exhibited far-from-equilibrium growth shapes at different substrate temperatures. Ni appeared to form a Ni(100)-like "dense" monolayer on the anisotropic $\mathrm{NiAl}(110)$ surface, which can be rationalized by the absence of lateral lattice mismatch between $\mathrm{Ni}(100)$ and $\mathrm{NiAl}(110)$. Deviation from perfect $\mathrm{Ni}(100)$ structure was also observed, based on the fact that the adlayer top surface was decorated by zig-zag shaped stripes. Prolonged deposition to achieve multilayer coverages revealed a transition from a $\mathrm{Ni}(100)$-like monolayer structure to a $\mathrm{Ni}(111)$-like structure.

Complementary theoretical analyses were carried out by collaborating scientists. In their work, traditional mean field (MF) theory was applied to analyze the island density at $200 \mathrm{~K}$. This analysis, and also an analysis of the island size distribution, suggested a contribution to the nucleation kinetics from heterogeneous nucleation - presumably at surface defects. Density functional theory (DFT) analysis was applied to provide insights into the observed growth behavior.
\end{abstract}

\section{Introduction}

Over the past few decades, scientists have carried out extensive experimental and theoretical analyses for both homoepitaxial and heteroepitaxial growth of metal thin films [13]. Many of these studies have focused on single-element single-crystal substrates (A on A 
homoepitaxy or A on B heteroepitaxy) [1,3]. Depositions on clean, ideal single-crystalline metal surfaces have led to a great understanding of the basic growth processes [1-6]. However, choosing instead intermetallics as substrates for thin film growth provides significant additional film structure and morphology possibilities. Until now, analyses of film growth on ordered alloy surfaces have been rare $(\mathrm{A}$ or $\mathrm{B}$ on $\mathrm{AB}$ or $\mathrm{CD})[7,8]$. There are significant complications for understanding growth on alloy substrates, such as distinct types of adsorption sites and diffusion pathways. These complications make the systems more difficult to deal with, but also much richer in possibilities for manipulation.

In this chapter of the thesis, we have chosen $\mathrm{NiAl}$ as a substrate to explore the possibilities mentioned above. The bcc-NiAl is of great technological interest because of its remarkable mechanical and thermal properties. The application of this alloy is widely developed in aeronautics (engine turbines) [9] and in microelectronics [10]. Moreover, thin layers of alumina that are well-ordered can be grown on this surface by oxidation at high temperature [11-14]. These layers can serve as support models in heterogeneous catalyst studies [12]. The NiAl low-index surfaces have also been well characterized. NiAl being a CsCl-type binary alloy, its (110) plane consists of rows of $\mathrm{Ni}$ and $\mathrm{Al}$ atoms in a 1:1 stoichiometry. The surface is bulk terminated except for two features. One is that the top layer $\mathrm{Al}$ atoms reside $0.02 \mathrm{~nm}$ higher than their in-plane Ni neighbors; the other is that the first interlayer spacing is a few percent larger than the deeper layers [15]. The surface unit cell of $\mathrm{NiAl}(110)$ can be envisioned as a rectangle with four $\mathrm{Ni}$ atoms at corners and one $\mathrm{Al}$ atom in the center (see Fig. 1).

There have been several relevant studies of $\mathrm{NiAl}(110)$ heteroepitaxial growth [7,8,16,17] and Ni homoepitaxial growth [18]. Previously in our group, we studied Ag thin 
film growth on $\mathrm{NiAl}(110)[7,17]$. This combination of materials is structurally intriguing because there is no lateral lattice mismatch between $\mathrm{Ag}(110)$ an $\mathrm{NiAl}(110)$ even though bulk structures of the Ni and NiAl are different. Our STM studies of Ag deposition on $\mathrm{NiAl}(110)$ in the temperature range from $200 \mathrm{~K}$ to $300 \mathrm{~K}$ revealed an initial bilayer growth mode. The film appeared to adopt the fcc $\mathrm{Ag}(110)$-like structure with linear depression and protrusion super feature decorating the top of Ag islands. For thicker Ag films, $\mathrm{Ag}(111)$-like monolayer growth dominated, forming more isotropic distorted hexagonal-shaped islands [17]. We proposed that the initial bilayer growth mode was driven by a quantum size effect (QSE) [16]. The NiAl(110) surface has a small depression in the density of states near the Fermi edge [19], and this feature serves to confine the electrons of the film material within the film. This spatial confinement creates a z-direction potential well that results in discrete energy states (particle in a 1D box model). This produces oscillations of total electronic energy with the height of the film. If the contribution of the electronic energy is significant, then some selected film heights will be energetically favorable [20].

Besides the previous $\mathrm{Ag} / \mathrm{NiAl}(110)$ heteroepitaxy study, there has been a low-energy electron microscopy study of $\mathrm{Al}$ deposition on $\mathrm{NiAl}(110)$ done by McCarty and coworkers [8]. Their experiments were performed in the high temperature range from $600 \mathrm{~K}$ to $900 \mathrm{~K}$. They examined the topography and composition of the NiAl(110) surface as it evolved during Al deposition. They observed motion of point and line defects within the substrate material [8]. They found two stages of film growth. Initially, as Al was deposited, NiAl layers grew. This growth mode was driven by the extraction of Ni atoms at surface antisite defects $[21,22]$ by the $\mathrm{Al}$ adatoms. Ejected $\mathrm{Ni}$ atoms reacted with $\mathrm{Al}$ adatoms and formed stable NiAl. However, as the surface became Al enriched, the second stage set in, which 
involved dislocation motion. The surface antisite $\mathrm{Ni}$ atoms were consumed by $\mathrm{Al}$ in the first stage, then Ni vacancies formed and, when sufficiently abundant, bulk Ni atoms started to move to the surface and bond with $\mathrm{Al}$ adatoms.

STM data is also available for Ni homoepitaxy on the Ni(100) surface [18]. Ni follows the Frank-van der Merwe (i.e. layer-by-layer) growth mode on the clean $\mathrm{Ni}(100)$ surface at $300 \mathrm{~K}$ (as it must, in the thermodynamic limit). Ni forms square-shaped islands reflecting the four-fold symmetry of the substrate. These islands grow laterally before coalescence.

In Sec. 2, background on our experimental settings and procedures is provided. In Sec. 3, the main experimental results of our study are presented. First, we describe the growth behavior of Ni films at room temperature. Second, discussions of the growth behavior at $200 \mathrm{~K}$ and $400 \mathrm{~K}$ are presented. In the third subsection, we discuss the postdeposition annealing experiment results. In the fourth subsection, we discuss the temperature dependence of nucleation and growth of Ni films and finally, in the last subsection, we briefly discuss the size distribution of the Ni islands. Also in Sec. 3, DFT energetics results from our collaborating group (consisting of Dr. James Evans and Dr. Yong Han) are presented to assist in explaining our experimental observations. Brief conclusions are provided in Sec. 4.

\section{Details of experiments}

Details of our UHV chamber setup, NiAl sample preparation are described in Refs. [7] and [17]. Ni was evaporated from a commercial Mantis e-beam evaporator. The base pressure was below $10^{-10}$ Torr during deposition. Flux calibration of the source was achieved 
by measuring the coverage directly from the STM images at submonolayer coverage. The crystallographic orientation of the $\mathrm{NiAl}(110)$ surface was determined by co-depositing $\mathrm{Ag}$ at $300 \mathrm{~K}$. Under these conditions, Ag forms elongated islands along the NiAl [001] direction [7] as shown in Fig. 2. Ni film composition and purity were checked with X-ray photoelectron spectroscopy.

\section{Results and discussion}

\subsection{Ni thin film growth at $300 \mathrm{~K}$}

Fig. 3 shows the STM data for Ni deposition on $\mathrm{NiAl}(110)$ at room temperature for various coverages. Deposition of $0.18 \mathrm{ML} \mathrm{Ni}$ at $300 \mathrm{~K}$ with flux of $3 \times 10^{-3} \mathrm{ML} / \mathrm{s}$ produces islands with a density of $(4.5 \pm 0.6) \times 10^{-3} \mathrm{~nm}^{-2}$ (based on a total number of 964 islands in a total area of $2.12 \times 10^{5} \mathrm{~nm}^{2}$ ) and a height of $0.234 \pm 0.011 \mathrm{~nm}$, which means that the islands are a single atom high (see Fig. 3a). Note that this height is expected to be $0.176 \mathrm{~nm}$ from bulk Ni (i.e. The separation between Ni (100) and (200) planes). The island height value is obtained from the line profiles (averaging over 44 islands, and two lines per island). Pixel height histograms are also taken to compare the island height value to that obtained from line profiles. They show an average island height value of $0.228 \pm 0.007 \mathrm{~nm}$. Representative line profiles and pixel height histogram are shown in Fig. 3i and 3e respectively.

The Ni islands exhibit irregular shapes with an average size of $40.4 \pm 2.4 \mathrm{~nm}^{2}$ at 0.18 ML coverage. However, the islands are somewhat elongated along the vertical direction in the STM images. The aspect ratio of these islands, $\mathrm{R}=\mathrm{Y} / \mathrm{X}$, is $(1.43 \pm 0.13): 1$, where $\mathrm{Y} / \mathrm{X}$ are the extension of the islands along the long/short axis parallel to the $[001] /[-110]$ directions respectively (see Fig. 4). This corresponds to elongation along the [001] direction, 
the same direction along which Ag islands are elongated, as shown in Fig. 3. Another interesting feature is that the islands tend to adopt diagonal step edges. This feature is amplified in a lower flux Ni deposition (see Fig. 3a inset).

To interpret these observations, we focus first on the adatom adsorption sites. From DFT calculated adsorption energies [23], it is clear that there are two types of adsorption sites for Ni adatoms: Type A, Ni-Ni short bridge site and type B, Al-Al short bridge site (see Fig. 5a). DFT energetics also show that a single, isolated Ni atom prefers A sites over B sites by about $0.15 \mathrm{eV}$, even though $\mathrm{Ni}$ sits at B sites in the NiAl bulk.. (The adsorption energy for a $\mathrm{Ni}$ atom at an A site is $4.65 \mathrm{eV}$, whereas at a B site it is $4.50 \mathrm{eV}$ [23].) However, DFT shows that the energy per adatom is lower for multiple adsorbed $\mathrm{Ni}$ atoms if they populate both $\mathrm{A}$ and $\mathrm{B}$ sites, and form a dense adlayer on top of $\mathrm{NiAl}(110)$. This is partly due to the strong diagonal nearest-neighbor $(\mathrm{NN})$ adatom interaction. (The monolayer binding energy per atom for pure A site population is $4.79 \mathrm{eV}$, for pure $\mathrm{B}$ site population it is $4.70 \mathrm{eV}$, and for both $\mathrm{A}$ and $\mathrm{B}$ sites it is $5.45 \mathrm{eV}$, which shows that the energy gain is larger [23].) Note that $\mathrm{Ni}$ adatoms occupying both A and B sites form a "dense" adlayer, which has a diamond-shaped surface unit cell with a side length of $0.250 \mathrm{~nm}$ as shown in Fig. 5a. This cell can be regarded as a distorted $\mathrm{Ni}(100)$ surface unit cell which is square-shaped with a length of $0.249 \mathrm{~nm}$. Therefore, we propose that $\mathrm{Ni}$ adatoms form a distorted Ni(100)-like dense adlayer on $\operatorname{NiAl}(110)$.

Second, we turn the focus on the Ni island growth shape (see Fig. 5b). We propose that this growth shape results from an interplay between thermodynamics and growth kinetics. 
Thermodynamically, DFT energetics shows that energies for diagonal steps are lower than other orientations $(0.91 \mathrm{eV} / \mathrm{nm}$ for diagonal steps and 1.09 and $1.07 \mathrm{eV} / \mathrm{nm}$ for horizontal and vertical steps respectively) [23]. Therefore, in equilibrium, the islands should be diamond shaped with edges parallel to the $[1,-1,1]$ and $[-1,1,1]$ directions.

Kinetically, periphery diffusion of edge atoms in stable clusters plays a crucial role in determining the island shape. At $300 \mathrm{~K}$, Ni adatoms encounter a large edge diffusion barrier along horizontal and vertical step edges $\left(\mathrm{E}_{\mathrm{e}}=0.85 \mathrm{eV}\right)$. However, diagonal step edge diffusion is facile, with a diffusion barrier of only $0.35 \mathrm{eV}$ [23]. Thus, horizontal and vertical steps grow out and disappear. Therefore, diagonal step edges are favored from both thermodynamic and kinetic aspects.

Kinetics also helps explain the tendency for vertical elongation of the Ni islands. DFT analysis shows that the kink-site Ehrlich-Schwoebel Effect (KESE) plays a role in controlling the Ni growth shape [24,25]. In KESE, adatoms have to pay an additional energy cost (above the energy barrier for diffusing along a smooth step) to hop over a kink (corner) site along the step. In this system, the adatoms on the diagonal step edge encounter a smaller KES barrier when they hop from a diagonal step edge on to a horizontal step, than from a diagonal step edge on to a vertical step [23]. Therefore, edge atoms are more easily fed to the top and bottom of the growing island, making it grow out along the vertical direction and hence become elongated vertically (see Fig. 5c).

Next, we show that the submonolayer Ni film does not adopt a perfect $\mathrm{Ni}(100)$ structure. This is evidenced by a zig-zag-shaped stripe feature decorating the top of the Ni islands (see Fig. 6a and 6b). The orientation of these stripes with respect to the substrate is shown in Fig. 6c. The segments adopt two major directions as shown in the histogram. The 
length of each segment of the zig-zag seems to be limited by the size of the island, i.e. each segment spans the width of the Ni island. The height of the segment is on average $0.019 \pm$ $0.008 \mathrm{~nm}$. Such features also occur in higher level Ni islands. We hypothesize that the stripes arise from strain between the $\mathrm{NiAl}(110)$ substrate and the $\mathrm{Ni}(100)$ film. Different linear super structures have been observed in Ag films on $\mathrm{NiAl}(110)$ [17], when they were attributed to strain relief.

Starting at 0.5 ML coverage, second layer Ni islands start to nucleate and grow on top of the first layer. This growth should be rather similar to $\mathrm{Ni}(100)$ homoepitaxy since the growth template is now a single atomic Ni(100)-like layer on NiAl(110). As shown in Fig. $3 \mathrm{~b}$, deposition at $300 \mathrm{~K}$ produces Ni second layer islands with a height of $0.210 \pm 0.010 \mathrm{~nm}$ (averaging from 49 islands, one line profile per island). Compared with the first Ni layer, the islands in the second layer have more well-defined shapes. They exhibit rectangular shape with a preference to elongate along the first $\mathrm{Ni}$ layer diagonal step edges, making them adopt two distinct orientations. The length to width aspect ratio is $(1.72 \pm 0.34): 1$ (sampling from 43 islands at 0.9 ML coverage).

Film growth exceeding $1 \mathrm{ML}$ coverage is shown in Fig. 3c and 3d. For thicker Ni films, islands grow laterally in size and coalesce as a result of growth (see the appendix for more STM images showing multilayer Ni film growth). Unlike layer-by-layer growth mode, the top $\mathrm{Ni}$ adlayer starts to nucleate and grow before the layer underneath covers the whole surface. This growth behavior makes the root-mean-square (RMS) roughness of the film increase with film thickness (see Fig. 7) Islands exhibit a constant growth behavior in terms of shape and orientation-diagonal step edges are favored. The observed film growth behavior is similar to that in $\mathrm{Ni}(100)$ homoepitaxy [18]. However, there appears to be a transition at 6 
ML coverage. Prolonged Ni deposition shows that ultimately, thick films on NiAl(110) develop a Ni(111)-like structure. STM of a 10.6 ML film shows that islands are more isotropic in shape compared with the elongated rectangular top layer islands observed for thinner films. Some island edges make angles of $120^{\circ}$, as expected in the $\mathrm{Ni}(111)$-like hexagonal structure. These features are shown in Fig. 8.

In order to understand the transition from the $\mathrm{Ni}(100)$-like structure to $\mathrm{Ni}(111)$-like hexagonal structure, a simple argument in terms of surface energy can be made. For fcc metals, typically their (111) surface has the lowest surface energy compared to other lowindex or vicinal surfaces, because the (111) surface is most close-packed. For Ni, its surface energy is $0.695 \mathrm{eV}$ per atom for the (111) compared to $0.969 \mathrm{eV}$ per atom for the (100) surface [26]. Therefore, in order to lower the total surface energy, as Ni is randomly deposited on the surface, the (111) orientation tends to be exposed to minimize the surface energy. A similar growth behavior has also been observed in $\mathrm{Ag}$ growth on $\mathrm{NiAl}(110)$ [17]. For a $80 \mathrm{ML}$ Ag film grown at room temperature, STM reveals that islands are more rounded than the highly elongated shape observed for thin films. $120^{\circ}$ facets are frequently present on the Ag multilayer surface. In terms of kinetics, one needs to realize that even though $\mathrm{Ni}(100)$ suffers little lateral lattice mismatch with the $\mathrm{NiAl}(110)$, the film is not a perfect $\mathrm{Ni}(100)$ film even at the beginning of the growth process as evidenced in the zig-zag stripe structure discussed before. The initial stripe feature could produce perturbations in film growth from the ideal $\mathrm{Ni}(100)$ structure. As the film grows thicker, this perturbation could naturally trigger the transition from distorted a $\mathrm{Ni}(100)$ structure to $\mathrm{Ni}(111)$ structure. 
3.2. Ni thin film growth at $200 \mathrm{~K}$ and $400 \mathrm{~K}$

In this subsection, we discuss the Ni film growth at temperatures other than $300 \mathrm{~K}$. Fig. 9 shows Ni submonolayer film growth at various temperatures. As shown in Fig. 9a, deposition of $0.22 \mathrm{ML} \mathrm{Ni}$ at $200 \mathrm{~K}$ with flux of $1.3 \times 10^{-3} \mathrm{ML} / \mathrm{s}$ produces islands with a density of $(5.1 \pm 0.2) \times 10^{-2} \mathrm{~nm}^{-2}$ (based on a total number of 1295 islands in a total area of $2.56 \times 10^{4} \mathrm{~nm}^{2}$ ), and a height of $0.207 \pm 0.008 \mathrm{~nm}$ (averaging over 92 islands, one line profile per island). The average island size is $4.35 \pm 0.2 \mathrm{~nm}^{2}$. Due to limited terrace diffusion, Ni film growth at $200 \mathrm{~K}$ results in a much higher island density and thus a significant smaller island size than at $300 \mathrm{~K}$. Also, the elongation along the $\mathrm{NiAl}$ [001] direction and the propensity for diagonal step edges seen at $300 \mathrm{~K}$ are not observed at $200 \mathrm{~K}$. Instead, islands exhibit irregular shape with no obvious preferential step orientation.

Fig. 9c shows deposition of $0.18 \mathrm{ML} \mathrm{Ni}$ at $400 \mathrm{~K}$ with a flux of $1.2 \times 10^{-3} \mathrm{ML} / \mathrm{s}$. The island density is $(1.5 \pm 0.2) \times 10^{-3} \mathrm{~nm}^{-2}$ (based on a total number of 471 islands on a total area of $3.10 \times 10^{5} \mathrm{~nm}^{2}$ ), with an average size of $114 \pm 7 \mathrm{~nm}^{2}$. The island height is $0.211 \pm 0.007$ nm. (Averaging over 33 islands, 3 line profiles per island) Ni islands appear to be 6-sided polygons with vertical and diagonal steps. To rationalize this growth behavior, similar reasoning can be applied as was used in the discussion of $300 \mathrm{~K}$ island growth shapes. Kinetically, as surface temperature increases to $400 \mathrm{~K}$, step diffusion along horizontal and vertical steps becomes active, potentially allowing straight steps to form. However, the absence of horizontal steps is due to KESE. The anisotropy in corner rounding causes horizontal steps to grow out (disappear). Note that this 6-sided distorted hexagonal shape is still a kinetic growth shape. In other words, it is not the equilibrium shape. 
3.3. Ni film postdeposition annealing results

We have performed postdeposition annealing experiments to investigate how film morphology changes in respond to surface temperature increase. Annealing experiments are performed for $200 \mathrm{~K}$ and $400 \mathrm{~K}$ depositions.

For $200 \mathrm{~K} \mathrm{Ni}$ deposition, upon subsequent annealing to $500 \mathrm{~K}$, the Ni film starts to equilibrate as shown in Fig. 10a. As surface temperature is raised, Ni adatoms are presumably detaching from smaller islands, diffusing across the $\mathrm{NiAl}(110)$ surface and attaching to larger islands, i.e., the Ostwald ripening [27] is taking place. The thermodynamic driving force of this coarsening behavior is to reduce the total system free energy associated with the island edges. As a result, the island density decreases and average island size increases. Also, islands start to establish quasi-equilibrium shapes. For $400 \mathrm{~K}$ Ni deposition, annealing at $500 \mathrm{~K}$ starts to produce shape equilibration as well. As shown in Fig. 10b. The islands develop more squared shapes, resembling metal (100) homoepitaxy.

\subsection{Temperature dependence of Ni nucleation and growth}

STM images in Fig. 9 clearly show that the Ni island density increases as surface temperature decreases. This growth behavior indicates that Ni grows far-from-equilibrium. As discussed already in the introduction to this thesis (Chapter 1), the classic picture of island nucleation and far-from-equilibrium growth is that atoms randomly impinge on the surface at a rate $\mathrm{F}$ and adsorb on it. Once the atoms adsorb on the surface and become adatoms, they diffuse on the terraces between neighboring adsorption sites at a rate $\mathrm{D}$ which is a function of surface temperature $\mathrm{T}$ and terrace diffusion barrier $\mathrm{E}_{\mathrm{d}}$. The formation of an island requires that multiple adatoms aggregate and nucleate on the terrace or at a trap site. A critical size i, 
is defined such that a cluster consists of $i+1$ adatoms is considered to be stable, meaning that this cluster will survive and grow in size instead of decaying back into individual adatoms. If surface temperature is low enough, $\mathrm{i}=1$ is operative, i.e., two adatoms are sufficient to form a stable island. This is usually called irreversible island formation.

In metal thin film epitaxial growth systems, often a transition from irreversible to reversible island formation (i $>1$ ) occurs as the temperature increases. The temperature at which this occurs (for a fixed flux) can be calculated from a simple formula for a quantity called the crossover factor, $\mathrm{Y}$, defined as:

$$
Y=(v / F) \exp \left[-\beta\left(E_{d}+3 / 2 E_{N N}\right)\right]
$$

Here, $v$ is the attempt frequency for hopping. It usually takes a value from $10^{11} / \mathrm{s}$ to $10^{13} / \mathrm{s}$. F is the deposition rate in units of $\mathrm{ML} / \mathrm{s} ; \beta=1 /\left(\mathrm{k}_{\mathrm{B}} \mathrm{T}\right)$ and $\mathrm{k}_{\mathrm{B}}$ is the Boltzmann constant; $\mathrm{E}_{\mathrm{d}}$ is the terrace diffusion barrier and $\mathrm{E}_{\mathrm{NN}}$ is the nearest neighbor bond energy. Specifically for $\mathrm{Ni}$ on $\mathrm{NiAl}(110)$, by using DFT to calculate the difference in adsorption energies for Ni adsorbed on an A site (see Sec. 3.1) and at the transition state (saddle point on potential energy surface), the value for $E_{d}$ is found to be $0.402 \mathrm{eV}$ [23] (see Fig. 11). Note that in spite of $\mathrm{NiAl}(110)$ being an anisotropic surface, the terrace diffusion is more isotropic, having the same diffusion barrier in both horizontal and vertical directions. $\mathrm{E}_{\mathrm{NN}}$ corresponds to the diagonal NN dimer (one atom sits on A site and the other atom sits on a nearest B site) bond energy, which is $0.19 \mathrm{eV}$ [23]. The temperature $\mathrm{T}_{\text {trans }}$, that describes the transition from $\mathrm{i}=1$ to $\mathrm{i}>1$, is roughly determined from the condition that $\mathrm{Y}=10$. For values of $\mathrm{E}_{\mathrm{d}}=0.402 \mathrm{eV}$, $\mathrm{E}_{\mathrm{NN}}=0.19 \mathrm{eV}, \mathrm{F}=10^{-3} \mathrm{ML} / \mathrm{s}$ and $v=10^{13} / \mathrm{s}$, this condition implies that $\mathrm{T}_{\text {trans }}=232 \mathrm{~K}$. This means that island formation is irreversible at $200 \mathrm{~K}$ and reversible at $300 \mathrm{~K}$ and $400 \mathrm{~K}$. 
Next, we turn to traditional mean-field rate equation theory to discuss the island density obtained from the STM studies at $200 \mathrm{~K}$. The MF rate equation theory was developed by Venables et.al. in the 1960s and is very successful in predicting the basic behavior of the island density for the case of isotropic terrace diffusion [5,6,28]. From this theory, the island density is given by:

$$
\mathrm{N}_{\mathrm{isl}} \sim \mathrm{K}(\theta)(\mathrm{F} / v)^{\chi} \exp (\beta \mathrm{E})
$$

where $\chi=\mathrm{i} /(\mathrm{i}+2)$ and $\mathrm{E}=\chi\left(\mathrm{E}_{\mathrm{d}}+\mathrm{E}_{\mathrm{i}} / \mathrm{i}\right)$. Here $\chi$ is a scaling factor and $\mathrm{E}_{\mathrm{i}}$ is the binding energy of clusters consisting of $\mathrm{i}$ atoms. Note that $\mathrm{E}_{1}=0$ (single adatom). If island formation is irreversible $(i=1)$, then $\chi=1 / 3$, and $E=E_{d} / 3$. For low coverage $\theta, K(\theta) \sim \theta^{1 /(i+2)}$. For values of $\mathrm{E}_{\mathrm{d}}=0.402 \mathrm{eV}, \mathrm{F}=1.3 \times 10^{-3} \mathrm{ML} / \mathrm{s}$ and $v=10^{13} / \mathrm{s}$, the theory gives an island density of $0.11 \mathrm{~nm}^{-2}$ or $6.5 \times 10^{-3} \mathrm{site}^{-1}$ for deposition at $200 \mathrm{~K}$. Note that this value is larger than that from our experiments $\left(5.1 \times 10^{-2} \mathrm{~nm}^{-2}\right.$ or $\left.3.0 \times 10^{-3} \mathrm{site}^{-1}\right)$. The lower experimental island density value might be a result of weak horizontal NN interaction.

It should be noted that one cannot simply apply the same calculation to estimate the island density for depositions at $300 \mathrm{~K}$ and $400 \mathrm{~K}$. For higher temperatures, as indicated by the crossover factor analysis, nucleation changes to being reversible already at $300 \mathrm{~K}$. This is partly due to the weak NN dimer interaction.

\subsection{Island size distribution analysis}

Last, we briefly discuss the island size distribution (ISD) for Ni film growth on $\mathrm{NiAl}(110)$. Fig. 12 shows the scaled ISD for Ni deposition at $200 \mathrm{~K}, 300 \mathrm{~K}$ and $400 \mathrm{~K}$. For homogeneous nucleation, increasing the temperature will increase the hopping rate $\mathrm{h}$ since $\mathrm{h}$ $=v \exp \left(-\mathrm{E}_{\mathrm{d}} / \mathrm{k}_{\mathrm{B}} \mathrm{T}\right)$. As a result, diffusion becomes more and more active, and deposited 
adatoms will preferentially find and be captured by a stable island. Thus, islands tend to have similar sizes. Therefore, as temperature increases, one would expect to have a narrower size distribution. However, our experimental observation contradicts this behavior. We propose that this is due to heterogeneous nucleation.

Heterogeneous nucleation occurs when diffusing adatoms get trapped by static surface defects or impurities [16,29]. $\mathrm{NiAl}(110)$ is known to have point defects where some $\mathrm{Ni}$ atoms reside on sites typically occupied by $\mathrm{Al}$ atoms. These sites are called $\mathrm{Ni}$ antisites $[21,22,30]$. These Ni antisites are found to act as trap sites for Ag adatoms and enhance nucleation [16]. We argue these point defects have the same effect on Ni island formation. If the density of these defects on the surface is high enough, it naturally will affect the adatom diffusion and nucleation process and hence a deviation from perfect homogeneous nucleation in island size distribution results.

As an aside, the presence of point defects on $\mathrm{NiAl}(110)$ surface also affects the $\mathrm{Ni}$ island density. The decrease in experimental island density with increasing temperature is slower than predicted by MF rate equation theory [23] for perfect homogeneous nucleation. This is shown in Fig. 13. The logarithmic scaled experimental island density shows a linear relationship with the inverse temperature, whereas a MF rate equation model will generate a curve that bends down when the temperature exceeds the transition temperature $T_{\text {trans }}$ due to reversible nucleation $(\mathrm{i}>1)$. The slower decrease in island density with increasing temperature can be attributed to the $\mathrm{Ni}$ antisites acting as trap sites for diffusing $\mathrm{Ni}$ adatoms. Thus, there are always a certain number of stable islands with a defect at the core. These islands may not contribute as a significant portion at low temperature. However, their presence causes a slower island density decrease as the temperature increases. 


\section{Conclusion}

In summary, we performed STM studies of Ni deposition on $\mathrm{NiAl}(110)$ in the temperature range from $200 \mathrm{~K}$ to $400 \mathrm{~K}$. Ni forms “dense" Ni(100)-like islands on NiAl(110) with a zig-zag shaped stripe feature which is probably due to strain relief. DFT analysis provides insights into the island growth shapes, which are rationalized by the thermodynamics and kinetics of the film growth process [23]. For thick Ni films (coverage exceeding $6 \mathrm{ML})$, a Ni(111)-like structure developed. Traditional MF theory is applied to analyze island density at $200 \mathrm{~K}$ [23]. Deviation from homogeneous nucleation behavior for island size distribution and island density reveals the presence of heterogeneous nucleation mediated by the $\mathrm{Ni}$ antisite point defects on $\mathrm{NiAl}(110)$ surface.

\section{References}

[1] J.W. Evans, P.A. Thiel, M.C. Bartelt, Surface Science Reports 61/1-2 (2006) 1.

[2] H. Brune, Surface Science Reports 31/4-6 (1998) 121.

[3] R.Q. Hwang, M.C. Bartelt, Chem. Rev. (Washington, D. C.) 97/4 (1997) 1063.

[4] E. Bauer, Appl. Surf. Sci. 11-12/1-3 (1982) 479.

[5] J.A. Venables, Philos Mag 27/3 (1973) 697.

[6] J.A. Venables, G.D.T. Spiller, M. Hanbucken, Rep Prog Phys 47/4 (1984) 399.

[7] Y. Han, B. Unal, F. Qin, D. Jing, C.J. Jenks, D.-J. Liu, P.A. Thiel, J.W. Evans, Phys Rev Lett 100/11 (2008) 116105/1.

[8] J.P. Pierce, N.C. Bartelt, K.F. McCarty, Phys Rev Lett 99/2 (2007) 026101. 
[9] V.K. Sikka, S.C. Deevi, S. Viswanathan, R.W. Swindeman, M.L. Santella, Intermetallics 8/9-11 (2000) 1329.

[10] P. Bartuska, J. Lasek, V. Paidar, Surface Engineering 19/3 (2003) 185.

[11] R.M. Jaeger, H. Kuhlenbeck, H.J. Freund, M. Wuttig, W. Hoffmann, R. Franchy, H. Ibach, Surf Sci 259/3 (1991) 235.

[12] J. Libuda, F. Winkelmann, M. Baeumer, H.J. Freund, T. Bertrams, H. Neddermeyer, K. Mueller, Surf Sci 318/1-2 (1994) 61.

[13] S.G. Addepalli, B. Ekstrom, N.P. Magtoto, J.S. Lin, J.A. Kelber, Surf Sci 442/3 (1999) 385 .

[14] R. Franchy, M. Wuttig, H. Ibach, Surf Sci 189-190 (1987) 438.

[15] H.L. Davis, J.R. Noonan, Phys Rev Lett 54/6 (1985) 566.

[16] Y. Han, B. Unal, D. Jing, F. Qin, C.J. Jenks, D.-J. Liu, P.A. Thiel, J.W. Evans, Phys. Rev. B: Condens. Matter 81/11 (2010) 115462/1.

[17] B. Unal, F. Qin, Y. Han, D.J. Liu, D.P. Jing, A.R. Layson, C.J. Jenks, J.W. Evans, P.A. Thiel, Phys Rev B 76/19 (2007).

[18] E. Kopatzki, S. Guenther, W. Nichtl-Pecher, R.J. Behm, Surf Sci 284/1-2 (1993) 154.

[19] S.C. Lui, M.H. Kang, E.J. Mele, E.W. Plummer, D.M. Zehner, Phys. Rev. B: Condens. Matter 39/18 (1989) 13149.

[20] M.C. Tringides, M. Jalochowski, E. Bauer, Phys. Today 60/4 (2007) 50.

[21] M.J. Mills, D.B. Miracle, Acta Metall. Mater. 41/1 (1993) 85.

[22] D.B. Miracle, Acta Metall. Mater. 41/3 (1993) 649.

[23] Y. Han, J.W. Evans, unpublished results.

[24] M. Li, J.W. Evans, Surf Sci 546/2-3 (2003) 127. 
[25] F. Rabbering, H. Wormeester, F. Everts, B. Poelsema, Phys. Rev. B: Condens. Matter $79 / 7$ (2009) 075402/1.

[26] L. Vitos, A.V. Ruban, H.L. Skriver, J. Kollar, Surf Sci 411/1-2 (1998) 186.

[27] W. Ostwald, Lehrbuch der allgemeinen Chemie, Leipzig, Germany, 1896.

[28] S. Stoyanov, D. Kashchiev, Curr. Top. Mater. Sci. 7 (1981) 69.

[29] G. Haas, A. Menck, H. Brune, J.V. Barth, J.A. Venables, K. Kern, Phys. Rev. B: Condens. Matter 61/16 (2000) 11105.

[30] A.J. Bradley, A. Taylor, Proc. R. Soc. London, Ser. A 159 (1937) 56. 


\section{Figure captions}

Fig. 1. Schematic presentation of $\mathrm{NiAl}(110)$ surface. Blue circles: Al top layer atoms; Yellow circles: Ni top layer atoms. Rectangle shows NiAl(110) surface unit cell.

Fig. 2. STM image of co-deposition of $\mathrm{Ni}$ and $\mathrm{Ag}$ on $\mathrm{NiAl}(110)$ at $300 \mathrm{~K}$. Ni deposition flux: $3 \times 10^{-3} \mathrm{ML} / \mathrm{s}$, Ni coverage: $0.2 \mathrm{ML}$. Ag deposition flux: $2.3 \times 10^{-2} \mathrm{ML} / \mathrm{s}, \mathrm{Ag}$ coverage: $1.4 \mathrm{ML}(0.7 \mathrm{BL})$. Images size: $60 \times 100 \mathrm{~nm}^{2}$. Tip bias: $+1 \mathrm{~V}$, tunneling current: $0.5 \mathrm{nA}$. The $\mathrm{NiAl}$ substrate, the $\mathrm{Ag}$ islands, the bare $\mathrm{Ni}$ islands and the $\mathrm{Ag}$ covered $\mathrm{Ni}$ islands are labeled as $\mathrm{NiAl}, \mathrm{Ag} / \mathrm{NiAl}, \mathrm{Ag} / \mathrm{Ni}$ and $\mathrm{Ni}$ respectively. The arrows show the $\mathrm{NiAl}[001]$ and [-110] directions.

Fig. 3. STM data for $\mathrm{Ni}$ deposited on $\mathrm{NiAl}(110)$ at $300 \mathrm{~K}$ as a function of coverage. Deposition flux F $=3 \times 10^{-3} \mathrm{ML} / \mathrm{s}$. The arrow points to the NiAl [001] direction. (a-d) show representative STM images of size of $50 \times 50 \mathrm{~nm}^{2}$. Tip bias: $+2 \mathrm{~V}$, tunneling current: $0.5 \mathrm{nA}$. Inset of (a) shows $300 \mathrm{~K} \mathrm{Ni}$ deposition with a flux $\mathrm{F}=3 \times 10^{-4} \mathrm{ML} / \mathrm{s}$. (e-h) show pixel height histograms from the above STM images. Peaks in the histograms are labeled with the island level (0 represents the substrate). (i-1) show representative line profiles for the levels indicated.

Fig. 4. Schematic showing the definition of the island aspect ratio, $\mathrm{R}=\mathrm{Y} / \mathrm{X}$. $\mathrm{X}, \mathrm{Y}$ are the extension of the island along the short/long axis parallel to the $[-110] /[001]$ directions respectively.

Fig. 5. Schematic presentation of Ni adlayer on $\mathrm{NiAl}(110)$. Blue circles: Al atoms on $\mathrm{NiAl}(110)$ top layer; Yellow circles: $\mathrm{Ni}$ atoms on $\mathrm{NiAl}(110)$ top layer; Red circles: $\mathrm{Ni}$ adatoms. (a) Ni adatoms occupying both $\mathrm{A}(\mathrm{Ni}-\mathrm{Ni}$ short bridge) and $\mathrm{B}$ (Al-Al short bridge) sites. Rectangle shows NiAl(110) surface unit cell. Diamond shows the 
distorted $\mathrm{Ni}(100)$ unit cell. (b) Illustration of a Ni island with horizontal, vertical and diagonal steps along the $\mathrm{NiAl}[001],[-110],[-111]$ and [1-11] directions respectively. (c) Illustration of KESE.

Fig. 6. Zig-zag stripe structure (a) and its pseudo 3D presentation (b). Image size: $20 \times 16$ $\mathrm{nm}^{2}$, tip bias: $+2 \mathrm{~V}$, tunneling current: $0.5 \mathrm{~nm}$. (c) Histogram showing the orientation of the zig-zag stripes with respect to the substrate. The inset shows how the angle is defined.

Fig. 7. Root-mean-square film roughness as a function of film thickness.

Fig. 8. Differentiated STM image of 10.6 ML Ni deposition on NiAl(110) at 300 K. Several sets of $120^{\circ}$ angles are illustrated with the white lines. Image size: $50 \times 50 \mathrm{~nm}^{2}$, tip bias: $-1 \mathrm{~V}$, tunneling current: $0.5 \mathrm{nA}$.

Fig. 9. STM data for 0.2 ML Ni deposited on $\mathrm{NiAl}(110)$ at various temperatures. The arrow points to the NiAl [001] direction. (a) $200 \mathrm{~K}$ deposition with $\mathrm{F}=1.3 \times 10^{-3} \mathrm{ML} / \mathrm{s}$. Tip bias: $+2 \mathrm{~V}$, tunneling current: $0.5 \mathrm{nA}$.(b) $300 \mathrm{~K}$ deposition with $\mathrm{F}=3 \times 10^{-3} \mathrm{ML} / \mathrm{s}$. Tip bias: $-2 \mathrm{~V}$, tunneling current: $0.5 \mathrm{nA}$. (c) $400 \mathrm{~K}$ deposition with $\mathrm{F}=1.2 \times 10^{-3}$ ML/s. Tip bias: $-1 \mathrm{~V}$, tunneling current: $0.5 \mathrm{nA}$. All STM images are $50 \times 50 \mathrm{~nm}^{2}$. (d-f) show pixel height histograms from the above STM images. Peaks in the histograms are labeled with the island level ( 0 represents the substrate). (g-i) show representative line profiles for the levels indicated.

Fig. 10. STM images of Ni films upon subsequent annealing at $500 \mathrm{~K}$ from (a) $200 \mathrm{~K}$ deposition with $\mathrm{F}=1.3 \times 10^{-3} \mathrm{ML} / \mathrm{s}$; (b) $400 \mathrm{~K}$ deposition with $\mathrm{F}=1.2 \times 10^{-3} \mathrm{ML} / \mathrm{s}$. Ni coverage for both cases is $0.2 \mathrm{ML}$, tip bias: $-1 \mathrm{~V}$, tunneling current: $0.5 \mathrm{nA}$. Image size: $50 \times 50 \mathrm{~nm}^{2}$ in (a) and $100 \times 100 \mathrm{~nm}^{2}$ in (b). 
Fig. 11. (a) Terrace diffusion path from MEP calculations for a $\mathrm{Ni}$ adatom on a $\mathrm{NiAl}(110)$ surface. (b) The corresponding energy curve.

Fig. 12. Scaled island size distribution for $0.2 \mathrm{ML} \mathrm{Ni} / \mathrm{Nial}(110)$ deposited at $200 \mathrm{~K}, 300 \mathrm{~K}$ and $400 \mathrm{~K}$.

Fig. 13. Experimental island density $\mathrm{N}_{\text {isl }}$ versus temperature $\mathrm{T}$. 
Figures

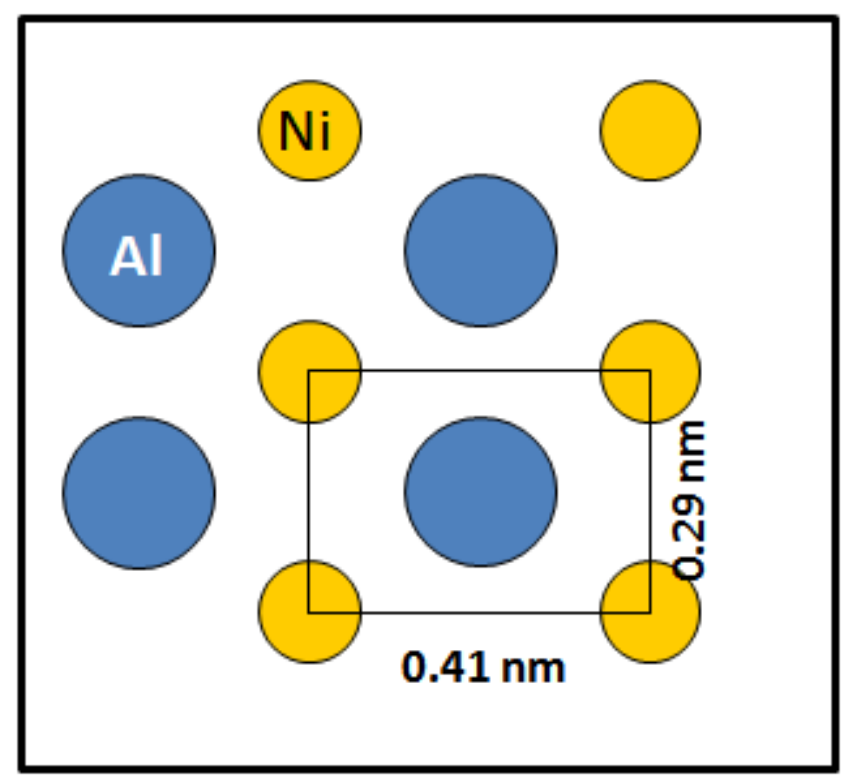

Fig. 1 


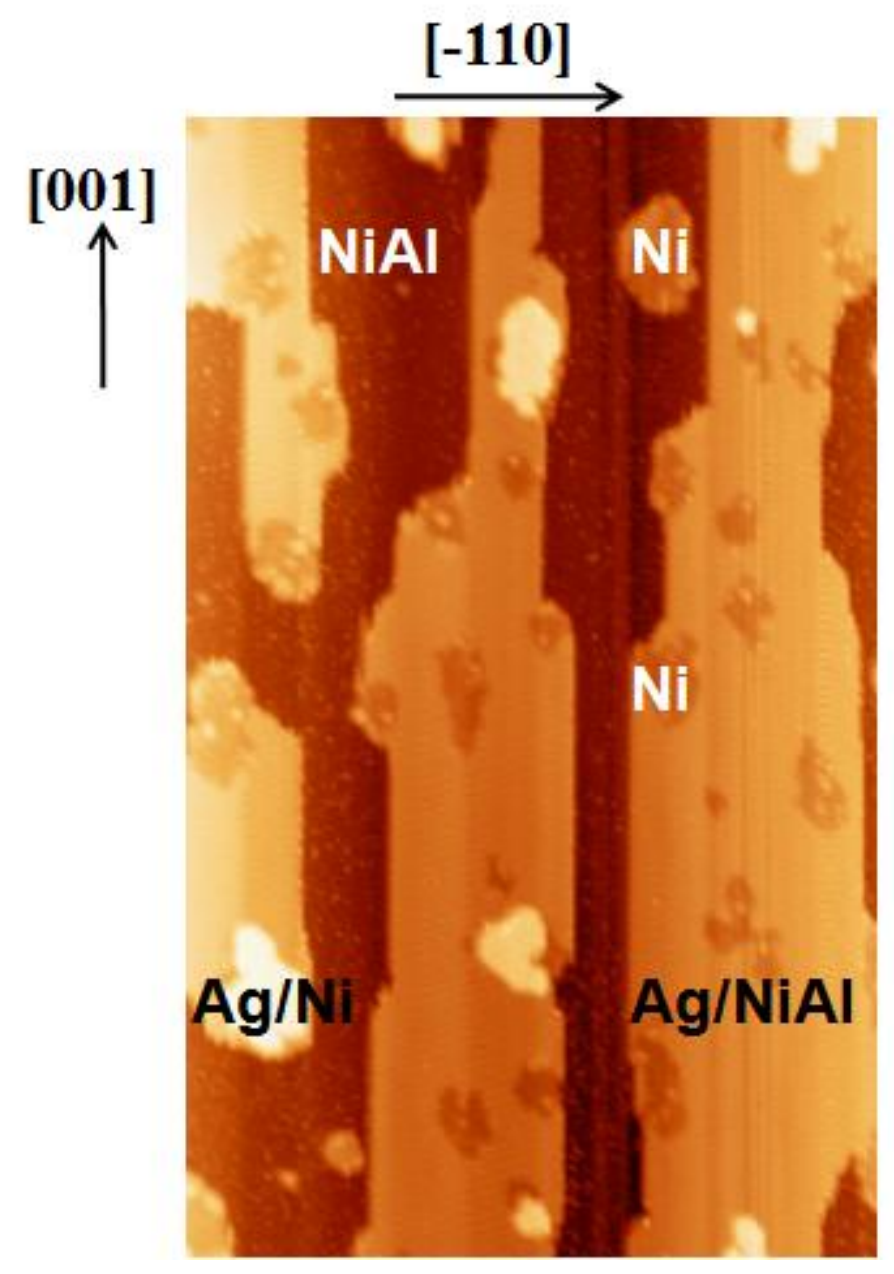

Fig. 2 

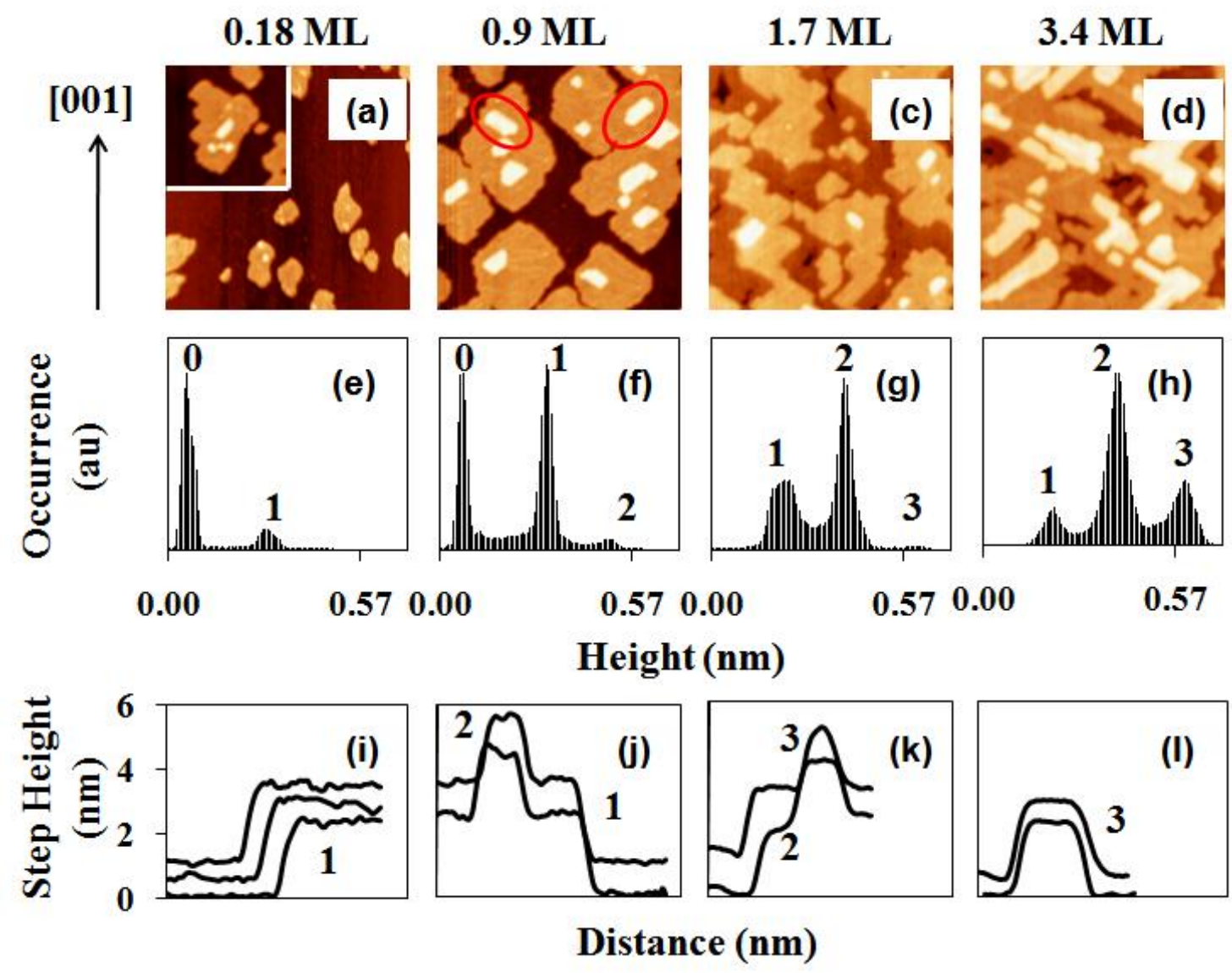

Fig. 3 


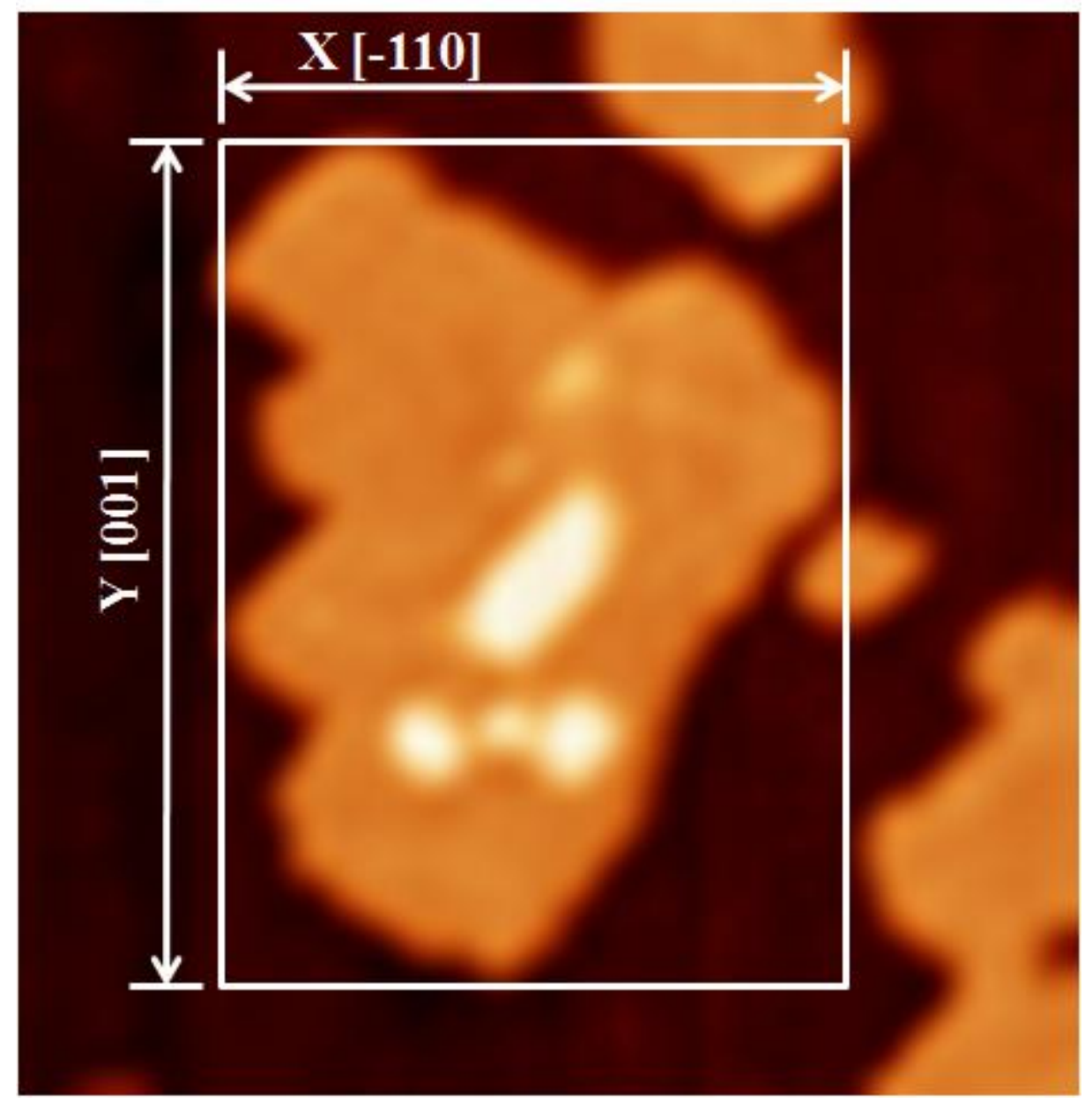

Fig. 4 


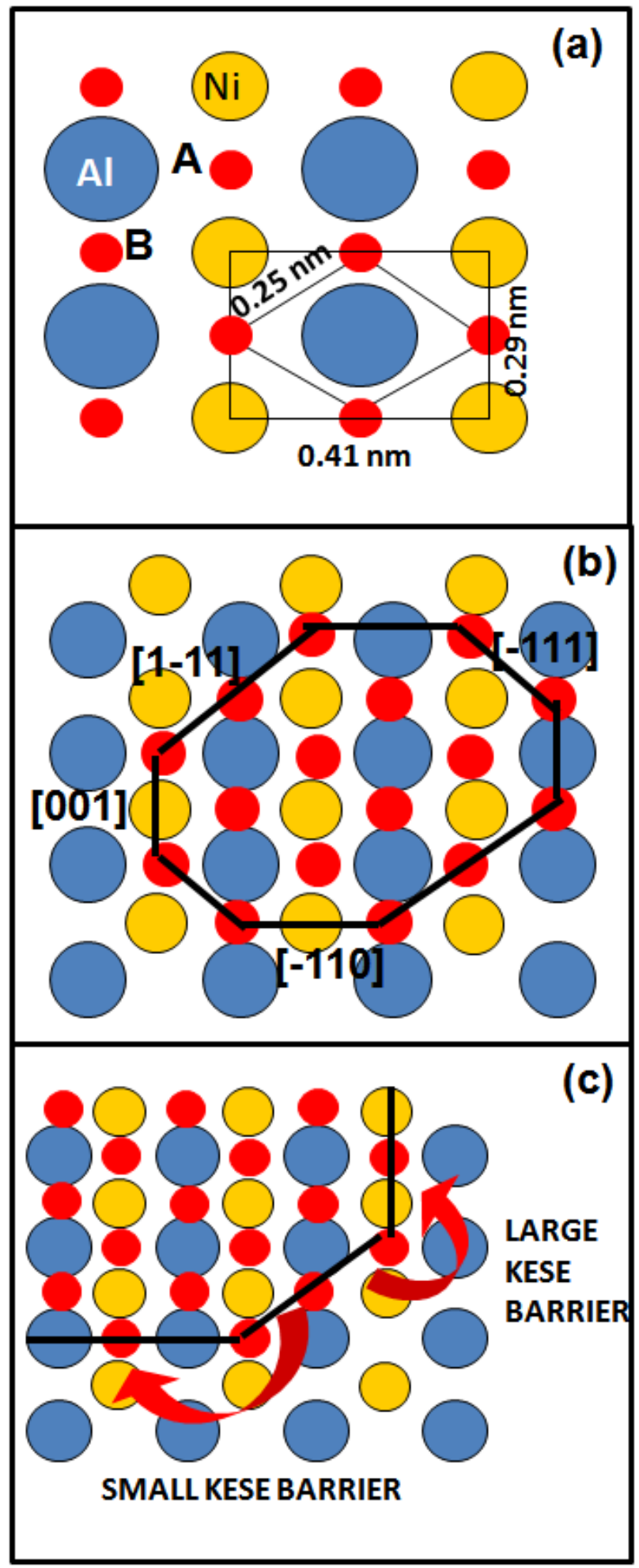

Fig. 5 

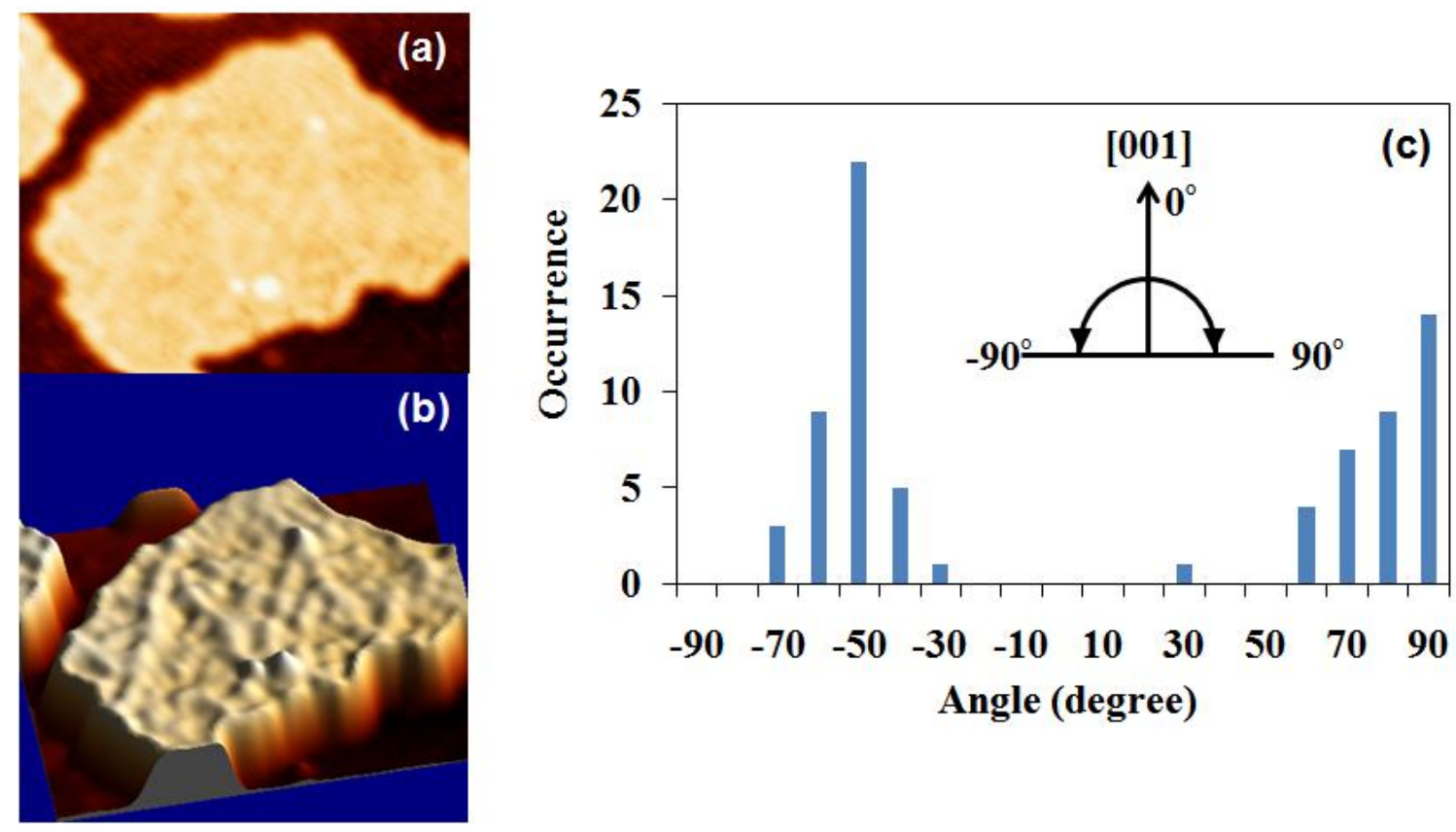

Fig. 6 


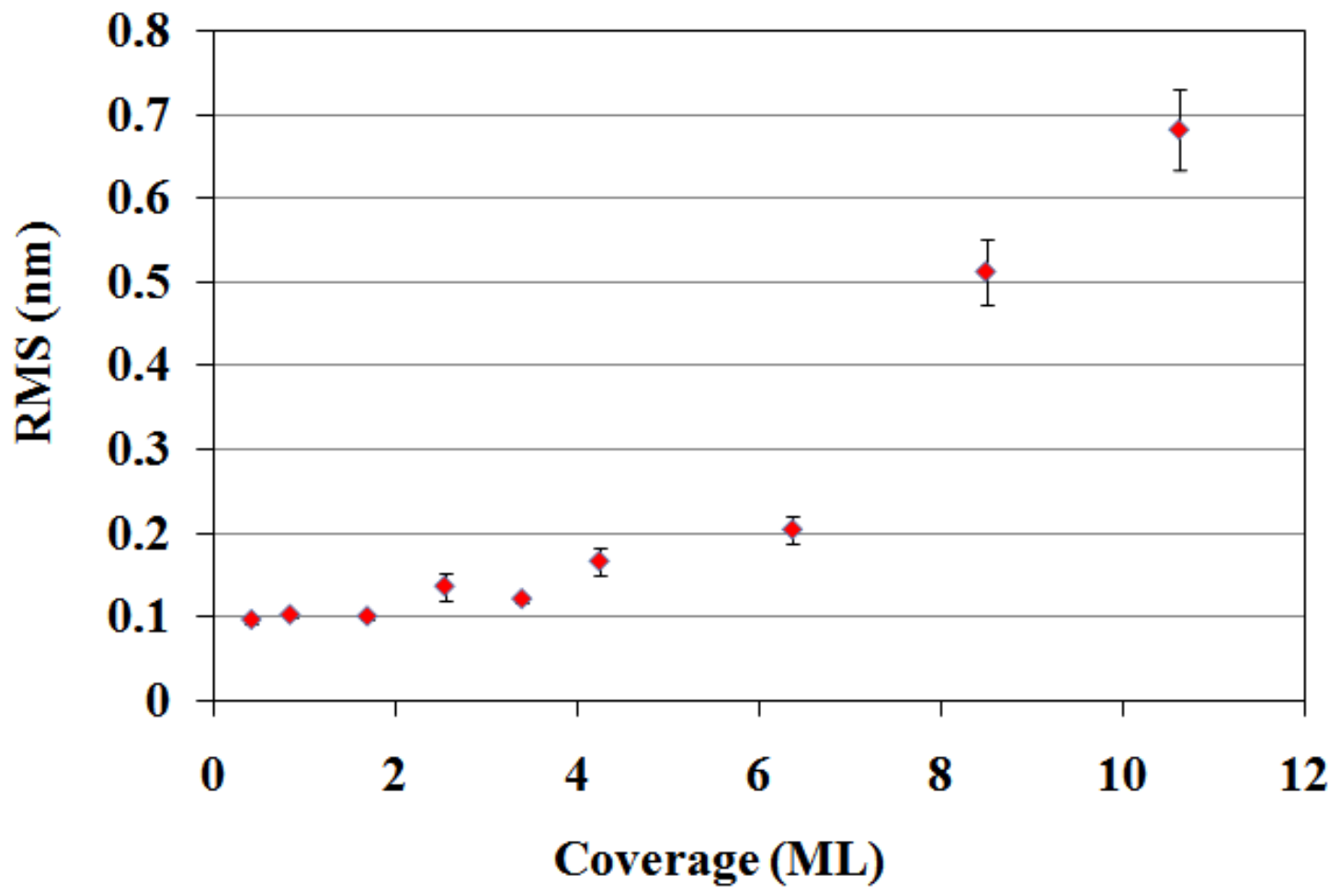

Fig. 7 


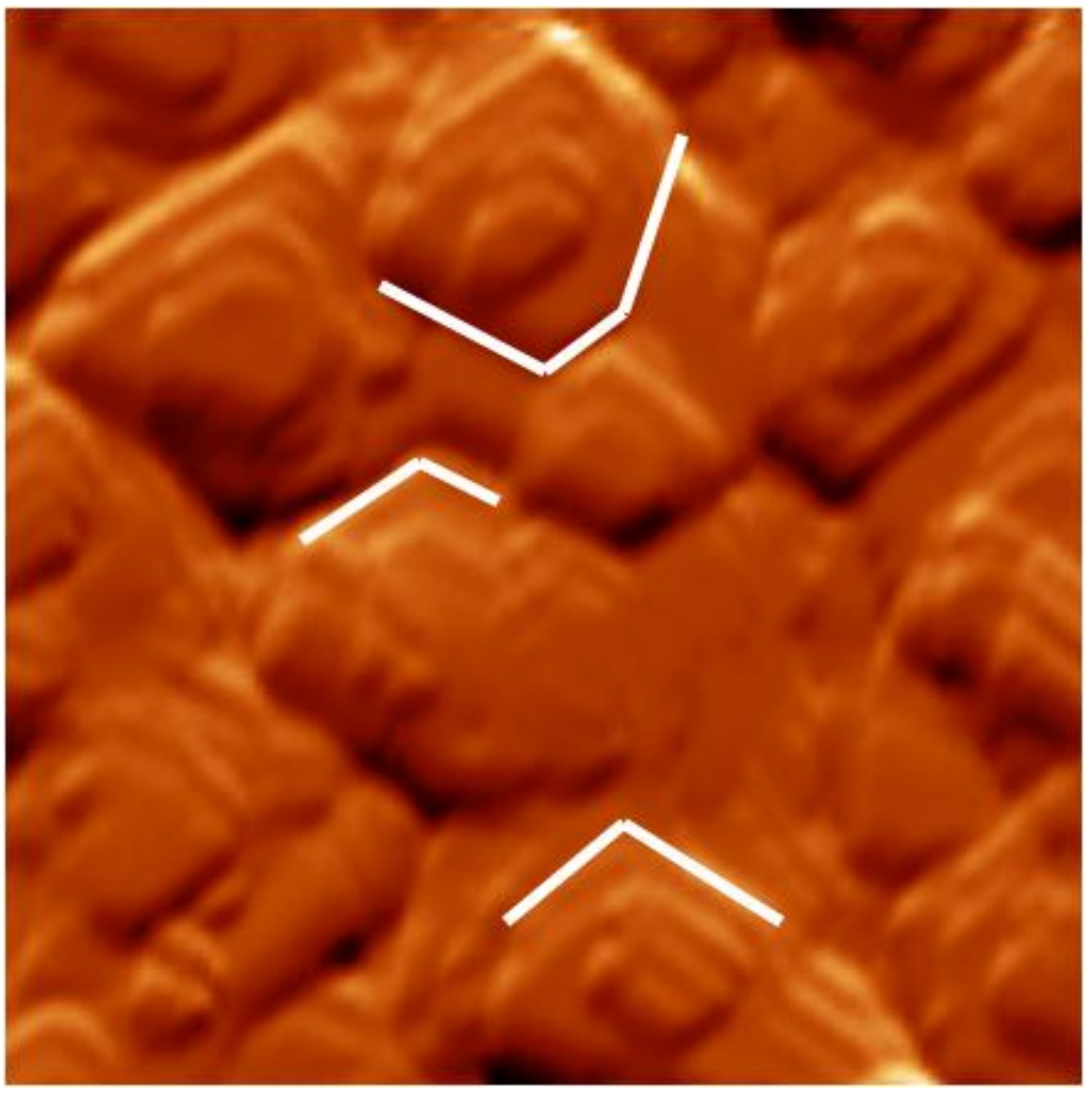

Fig. 8 


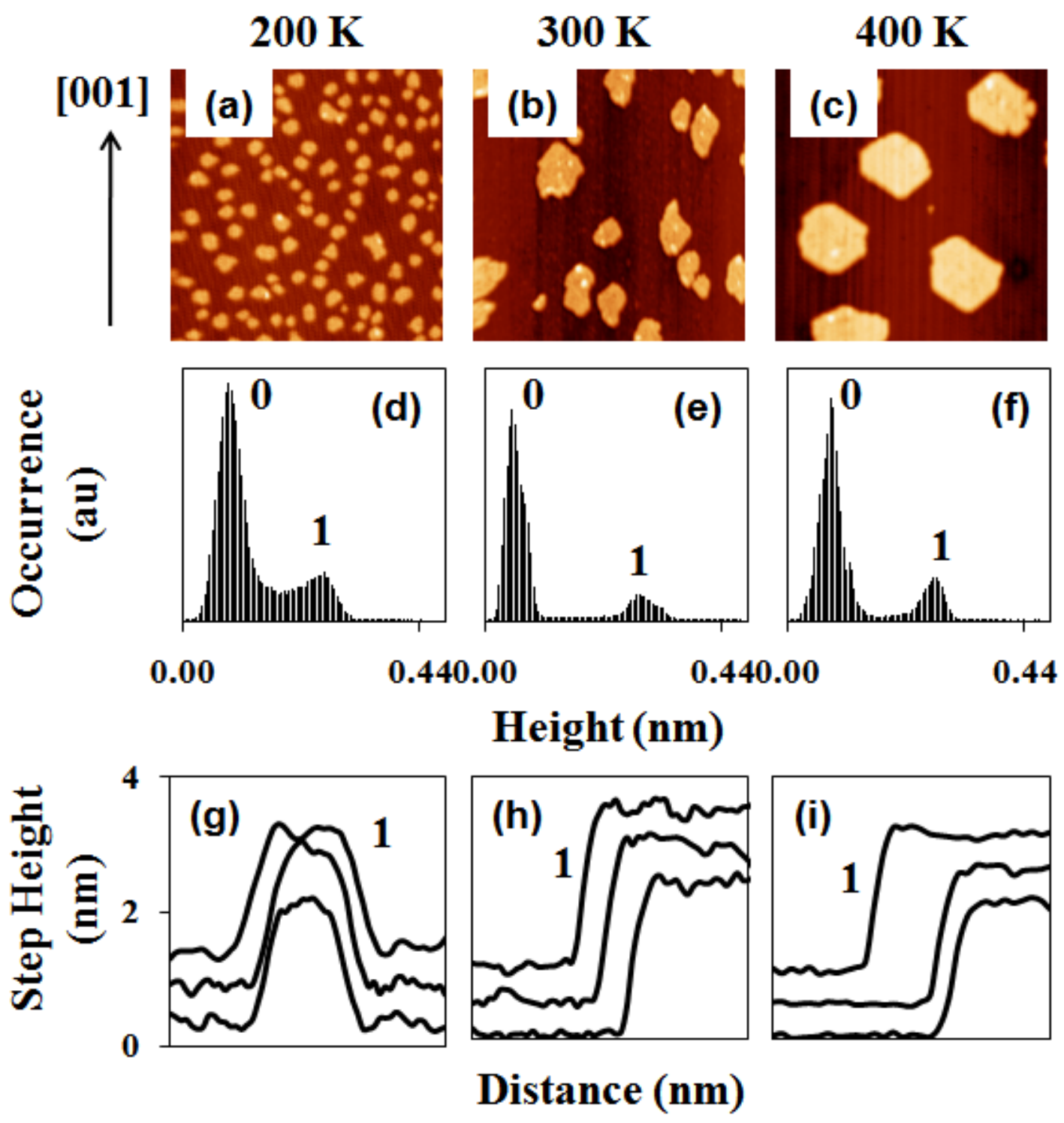

Fig. 9 


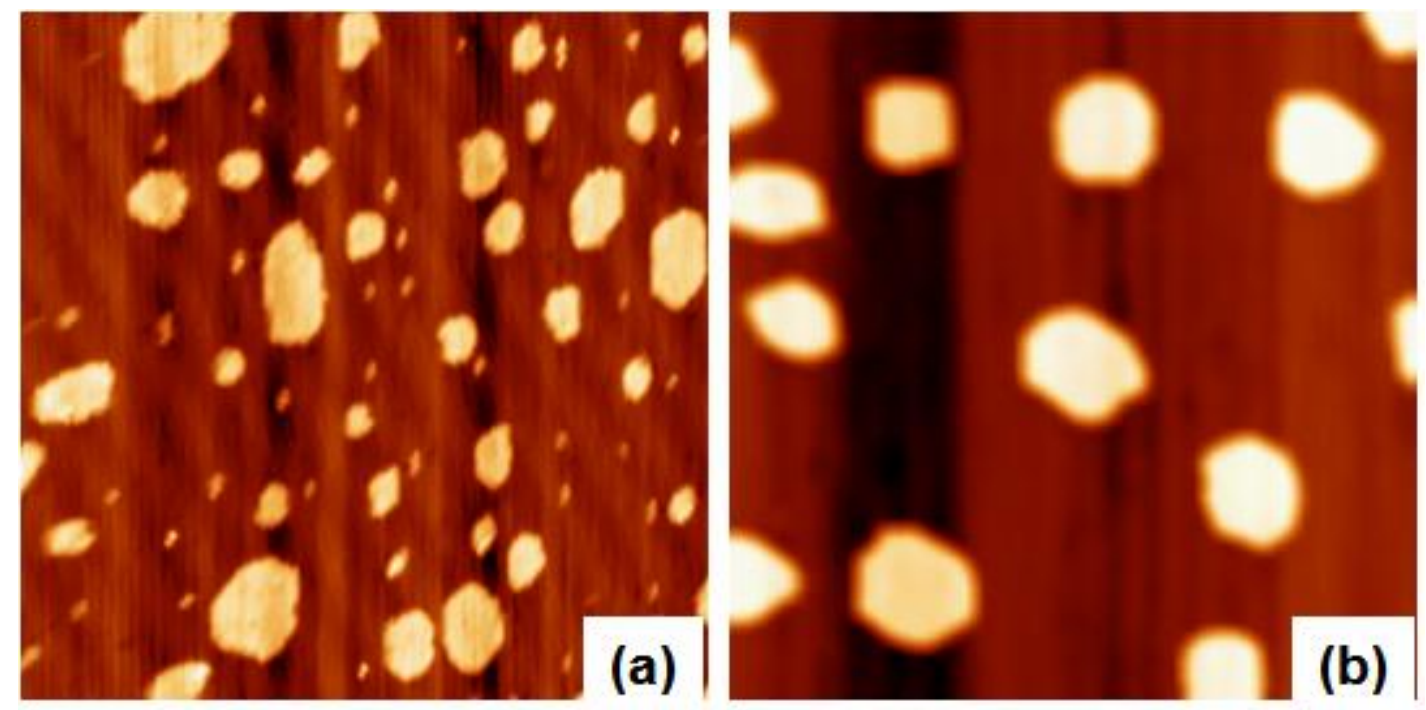

Fig. 10 

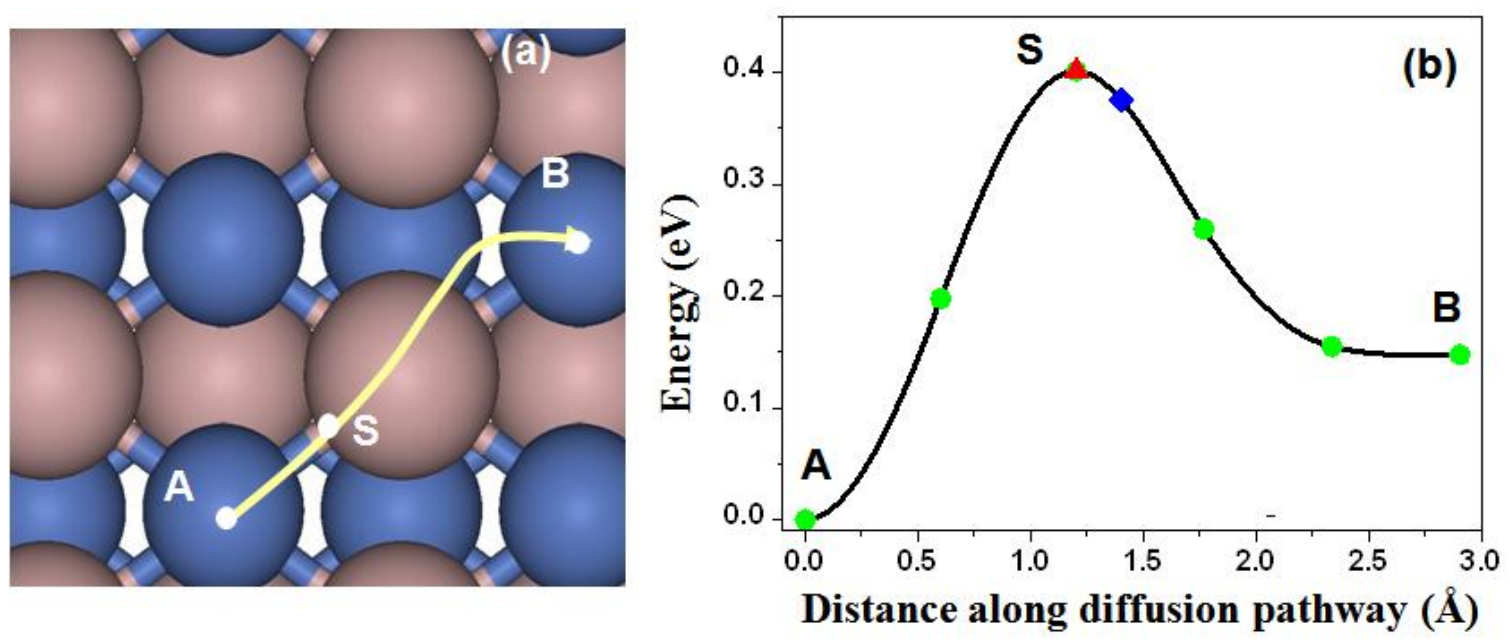

Fig. 11 

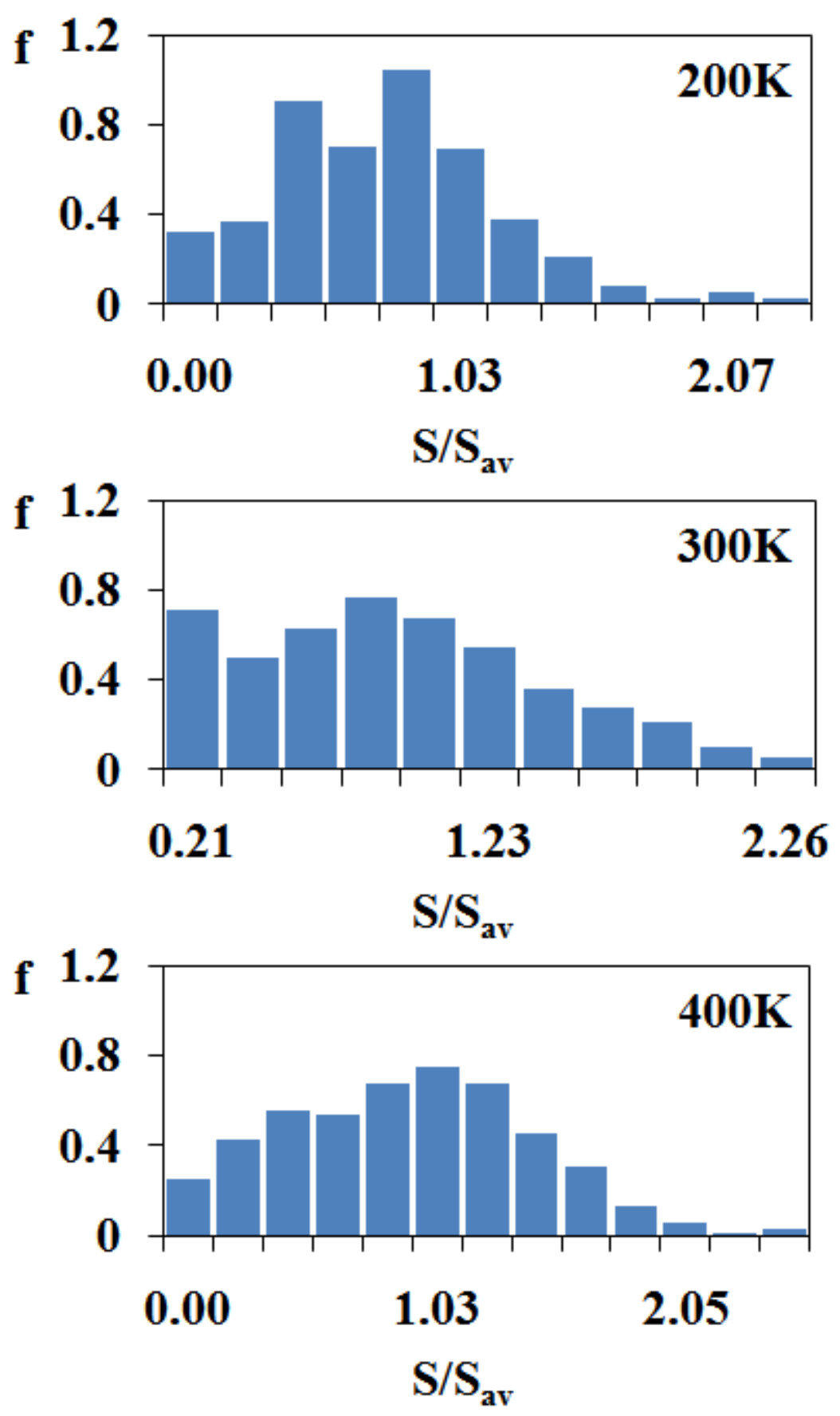

Fig. 12 


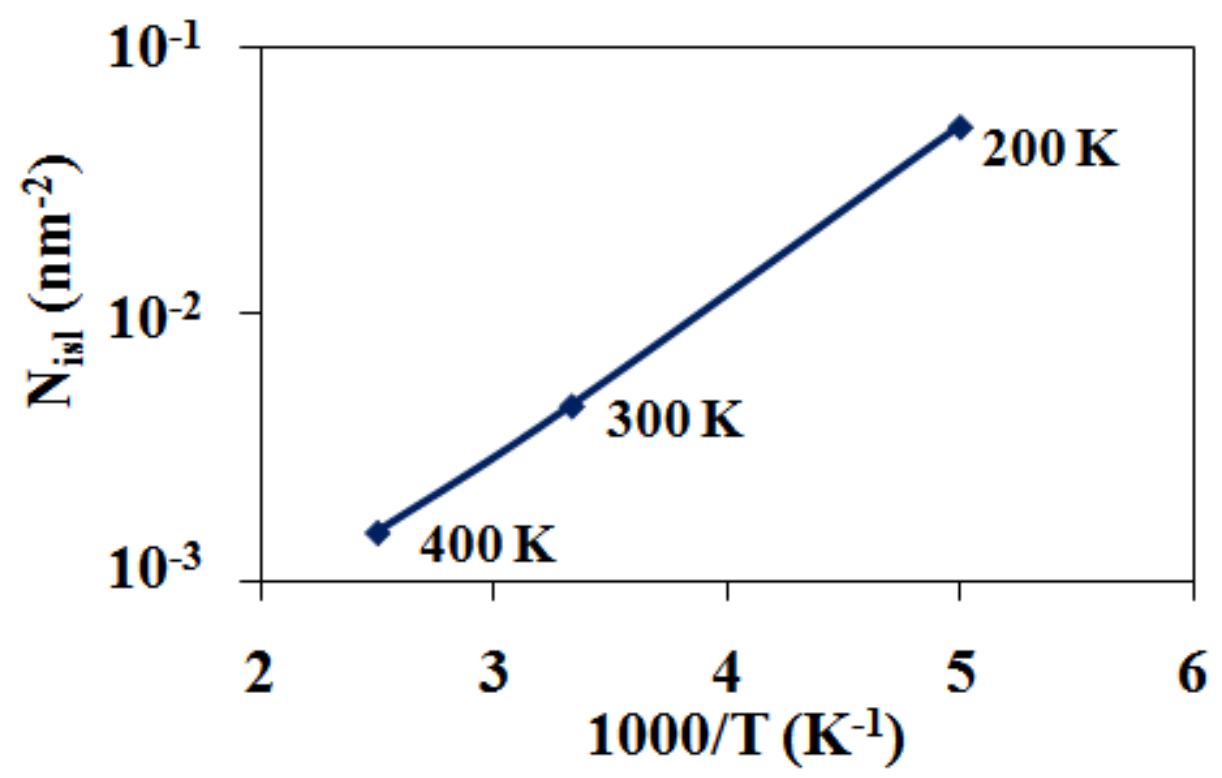

Fig. 13 


\section{APPENDIX. ADDITIONAL MATERIALS FOR CHAPTER 5}

Here we present additional, relevant data concerning the Ni thin film growth on $\mathrm{NiAl}(110)$. This appendix includes additional STM images from Ni depositions at different temperatures and additional STM data for postdeposition annealing experiments.

\section{$200 \mathrm{~K}$ Ni deposition}

Here we present additional STM images for $200 \mathrm{~K} \mathrm{Ni} \mathrm{deposition.} \mathrm{The} \mathrm{experimental}$ deposition flux is $1.3 \times 10^{-3} \mathrm{ML} / \mathrm{s}$. The coverage is $0.22 \mathrm{ML}$. Fig. A1 shows sample STM images of different scales.

\section{$300 \mathrm{~K}$ Ni deposition (submonolayer coverages)}

Here we present $300 \mathrm{~K} \mathrm{Ni}$ deposition with different flux values at submonolayer

coverage regime. Fig. A2 shows sample STM images for deposition with F $=3 \times 10^{-4} \mathrm{ML} / \mathrm{s}$. Fig A3 shows sample STM images for deposition with $\mathrm{F}=3 \times 10^{-3} \mathrm{ML} / \mathrm{s}$.

\section{$300 \mathrm{~K}$ Ni deposition (multilayer coverages)}

Result for multilayer Ni film growth at $300 \mathrm{~K}$ is shown here. The deposition flux is $\mathrm{F}$ $=1 \times 10^{-3} \mathrm{ML} / \mathrm{s}$. Ni coverage spans from 0.4 ML to 10.6 ML. Sample STM images are shown in Fig. A4.

\section{$400 \mathrm{~K}$ Ni deposition}

Result for $400 \mathrm{~K} \mathrm{Ni}$ deposition is presented here. The deposition flux is $\mathrm{F}=1 \times 10^{-3}$ ML/s. Ni coverage spans from 0.06 ML to 0.18 ML. Sample STM images are shown in Fig. A5. 


\section{Postdeposition annealing experiment for $200 \mathrm{~K}$ deposition}

We have performed postdeposition annealing experiments to investigate how Ni film morphology changes in response to surface temperature increase. After deposition at $200 \mathrm{~K}$, the sample has been heated to $300 \mathrm{~K}, 400 \mathrm{~K}$ and $500 \mathrm{~K}$. The sample is held at the elevated temperatures during STM scanning. Sample STM images are shown in Fig. A6.

\section{Postdeposition annealing experiment for $400 \mathrm{~K}$ deposition}

Postdeposition annealing experiments have also been performed for $400 \mathrm{~K} \mathrm{Ni}$

deposition. After deposition at $400 \mathrm{~K}$, the sample has been heated to $450 \mathrm{~K}$ and $500 \mathrm{~K}$. The sample is held at the elevated temperatures during STM scanning. Sample STM images are shown in Fig. A7. 


\section{Figure captions}

Fig. A1. STM images for $200 \mathrm{~K} \mathrm{Ni}$ deposition. $\mathrm{F}=1.3 \times 10^{-3} \mathrm{ML} / \mathrm{s}$. The coverage is 0.22

ML. Tip bias: $-1 \mathrm{~V}$, tunneling current: $0.5 \mathrm{nA}$. (a) $200 \times 200 \mathrm{~nm}^{2}$ (b) $100 \times 100 \mathrm{~nm}^{2}$ (c) $50 \times 50 \mathrm{~nm}^{2}$ (d) $25 \times 25 \mathrm{~nm}^{2}$.

Fig. A2. STM images for $300 \mathrm{~K} \mathrm{Ni}$ deposition. $\mathrm{F}=3 \times 10^{-4} \mathrm{ML} / \mathrm{s}$. Image size: $100 \times 100 \mathrm{~nm}^{2}$

. Tip bias: +2 V, tunneling current: $0.5 \mathrm{nA}$. (a) $0.03 \mathrm{ML}$ (b) $0.06 \mathrm{ML}$ (c) $0.12 \mathrm{ML}$ (d) $0.24 \mathrm{ML}$ (e) $0.48 \mathrm{ML}$ (f) $0.9 \mathrm{ML}$.

Fig. A3. STM images for $300 \mathrm{~K}$ Ni deposition. $\mathrm{F}=3 \times 10^{-3} \mathrm{ML} / \mathrm{s}$. Image size: $100 \times 100 \mathrm{~nm}^{2}$ . Tip bias: $+2 \mathrm{~V}$, tunneling current: $0.5 \mathrm{nA}$. (a) $0.03 \mathrm{ML}$ (b) 0.09 ML (c) 0.14 ML (d) 0.2 ML (e) 0.37 ML (f) 0.74 ML.

Fig. A4. STM images for $300 \mathrm{~K}$ Ni deposition. $\mathrm{F}=1 \times 10^{-3} \mathrm{ML} / \mathrm{s}$. Image size: $100 \times 100 \mathrm{~nm}^{2}$ . Tip bias: $-1 \mathrm{~V}$, tunneling current: $0.5 \mathrm{nA}$. (a) $0.43 \mathrm{ML}$ (b) $0.85 \mathrm{ML}$ (c) $1.7 \mathrm{ML}$ (d) 2.6 ML (e) 3.4 ML (f) 4.2 ML (g) 6.4 ML (h) 8.5 ML (i) 10.6 ML .

Fig. A5. STM images for $400 \mathrm{~K} \mathrm{Ni}$ deposition. $\mathrm{F}=1 \times 10^{-3} \mathrm{ML} / \mathrm{s}$. Image size: (a,c,e) $200 \times$ $200 \mathrm{~nm}^{2}(\mathrm{~b}, \mathrm{~d}, \mathrm{f}) 100 \times 100 \mathrm{~nm}^{2}$. Tip bias: $+2 \mathrm{~V}$, tunneling current: $0.5 \mathrm{nA}$. (a,b) 0.06 ML (c,d) 0.12 ML (e,f) 0.18 ML.

Fig. A6. STM images for $200 \mathrm{~K} \mathrm{Ni}$ deposition (a) followed by postdeposition annealing to $300 \mathrm{~K}(\mathrm{~b}), 400 \mathrm{~K}(\mathrm{c})$ and $500 \mathrm{~K}(\mathrm{~d}) . \mathrm{F}=1 \times 10^{-3} \mathrm{ML} / \mathrm{s}$. Ni coverage: $0.22 \mathrm{ML}$. Image size: $50 \times 50 \mathrm{~nm}^{2}$. Tip bias: $-1 \mathrm{~V}$, tunneling current: $0.5 \mathrm{nA}$.

Fig. A7. STM images for $400 \mathrm{~K} \mathrm{Ni}$ deposition (a) followed by postdeposition annealing to $450 \mathrm{~K}(\mathrm{~b})$ and $500 \mathrm{~K}(\mathrm{c}) . \mathrm{F}=1 \times 10^{-3} \mathrm{ML} / \mathrm{s}$. Ni coverage: $0.18 \mathrm{ML}$. Image size: $200 \times$ $200 \mathrm{~nm}^{2}$. Tip bias: $+2 \mathrm{~V}$, tunneling current: $0.5 \mathrm{nA}$. 
Figures
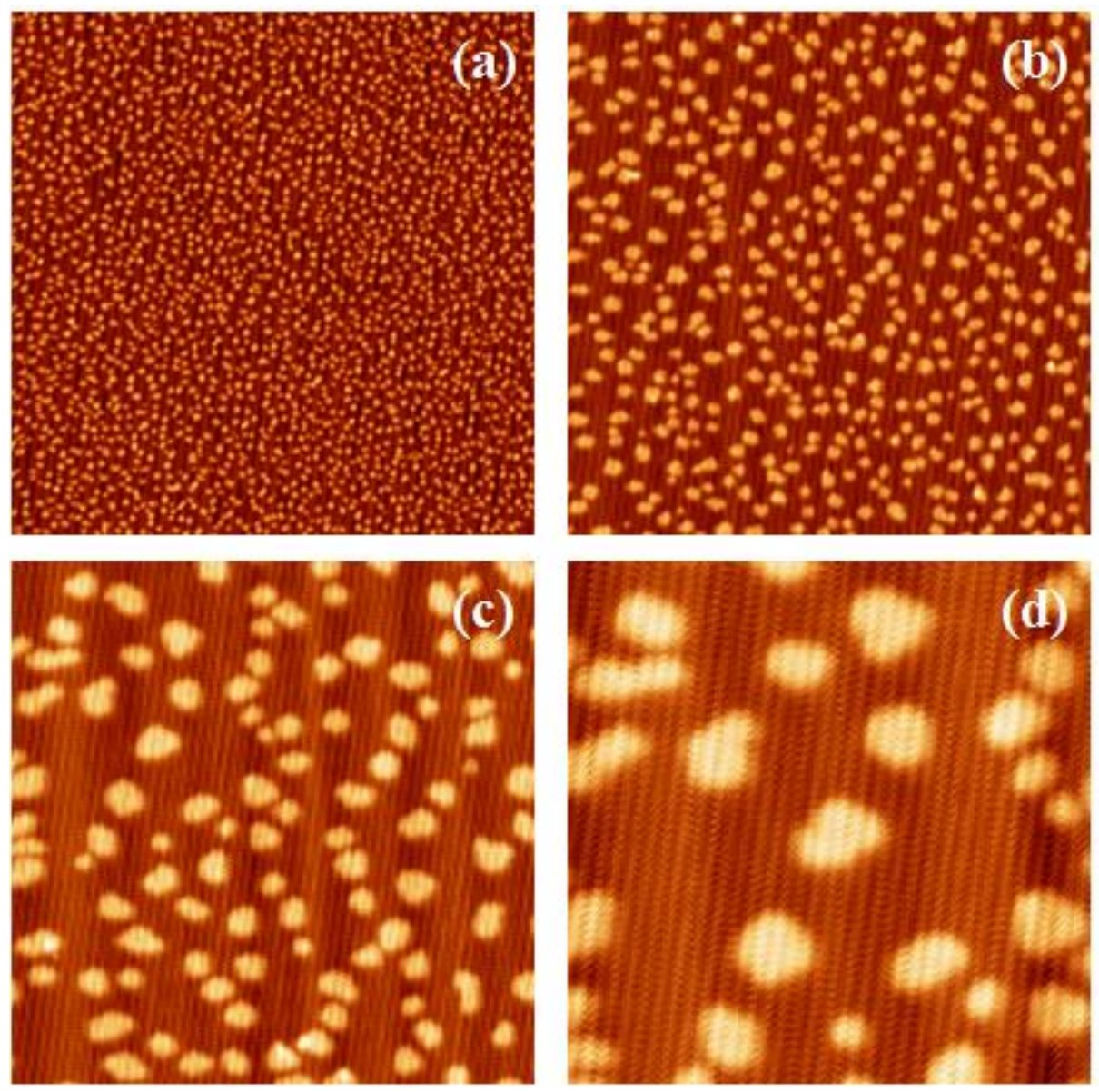

Fig. A1 

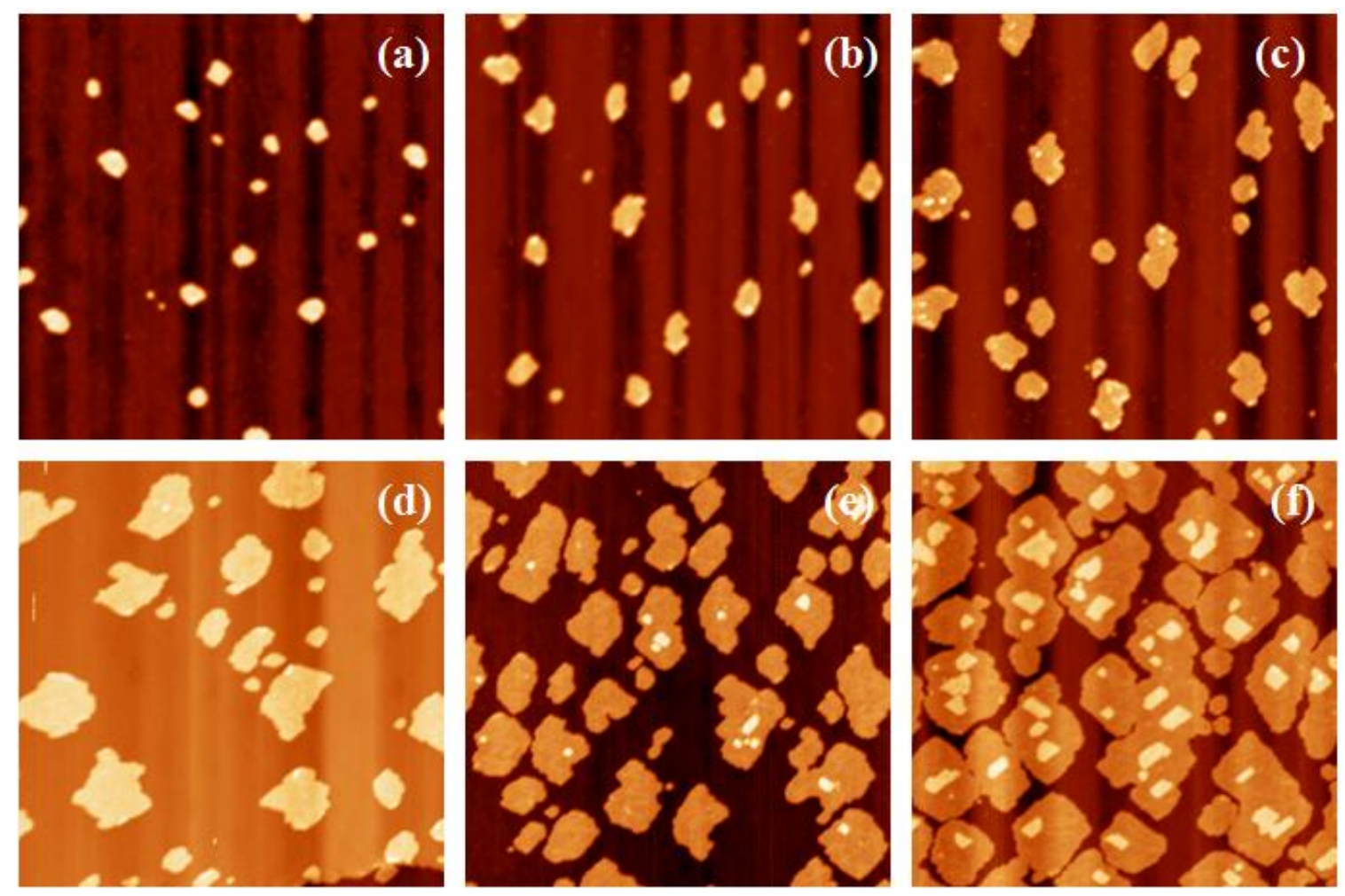

Fig. A2 


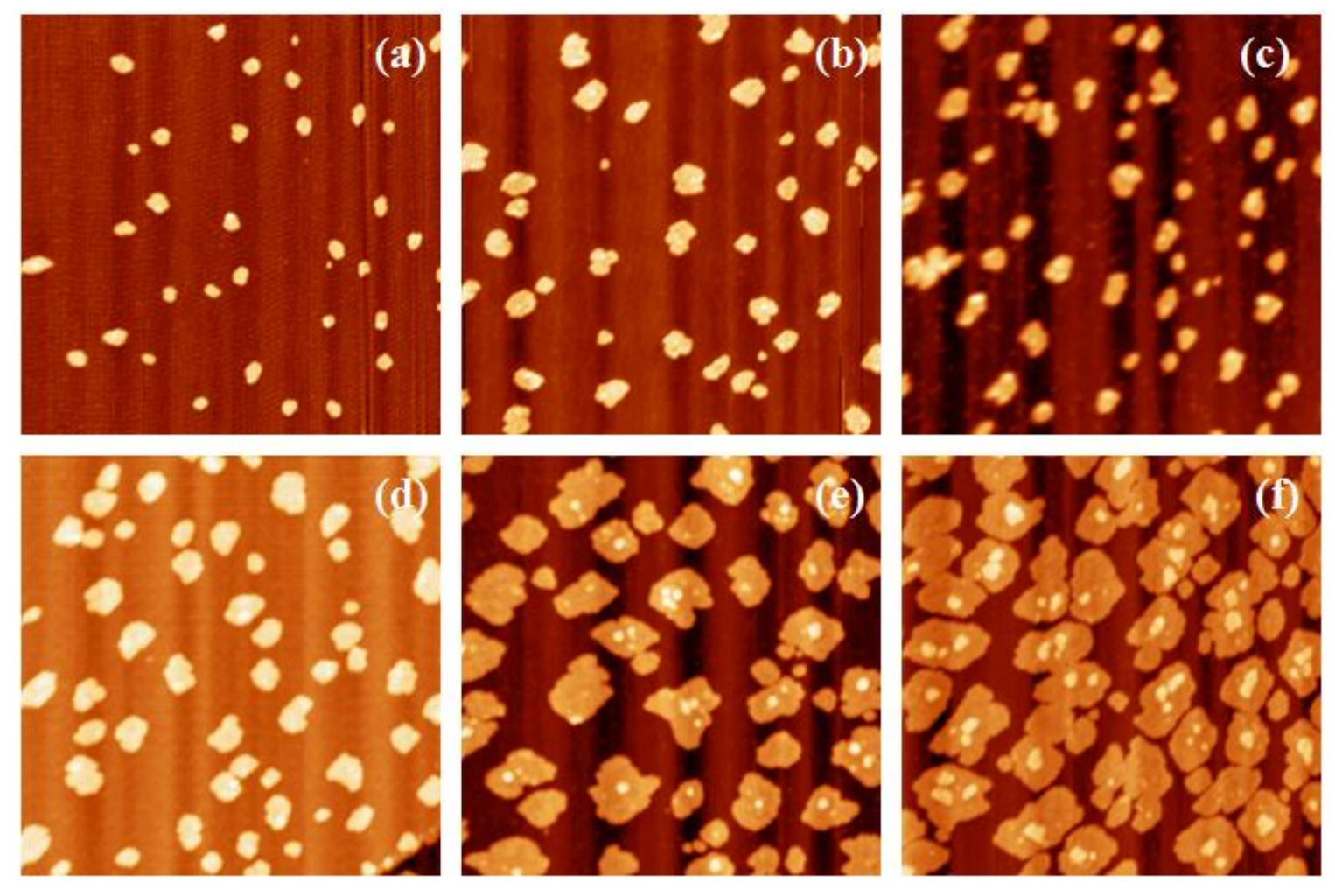

Fig. A3 

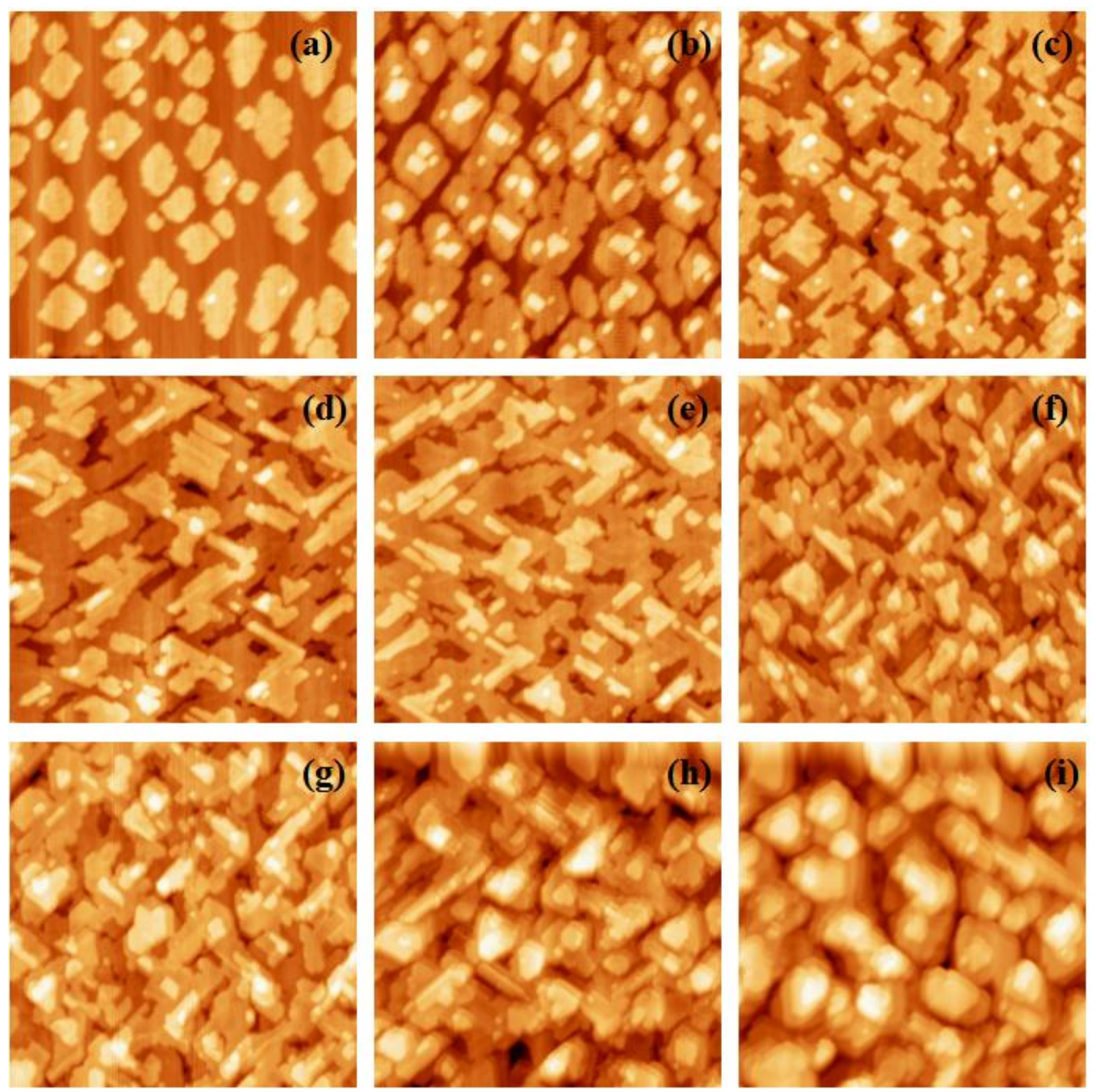

Fig. A4 

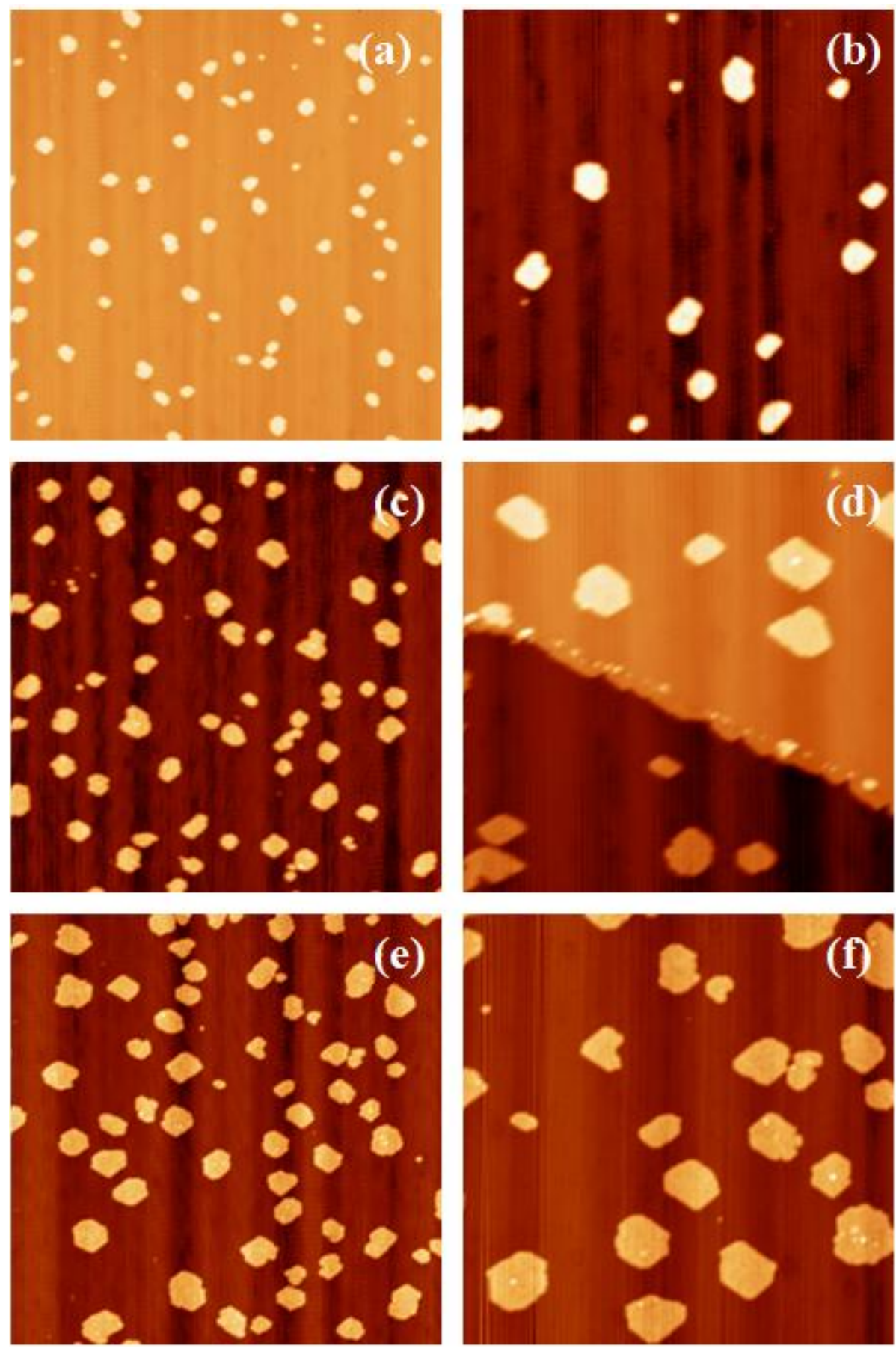

Fig. A5 

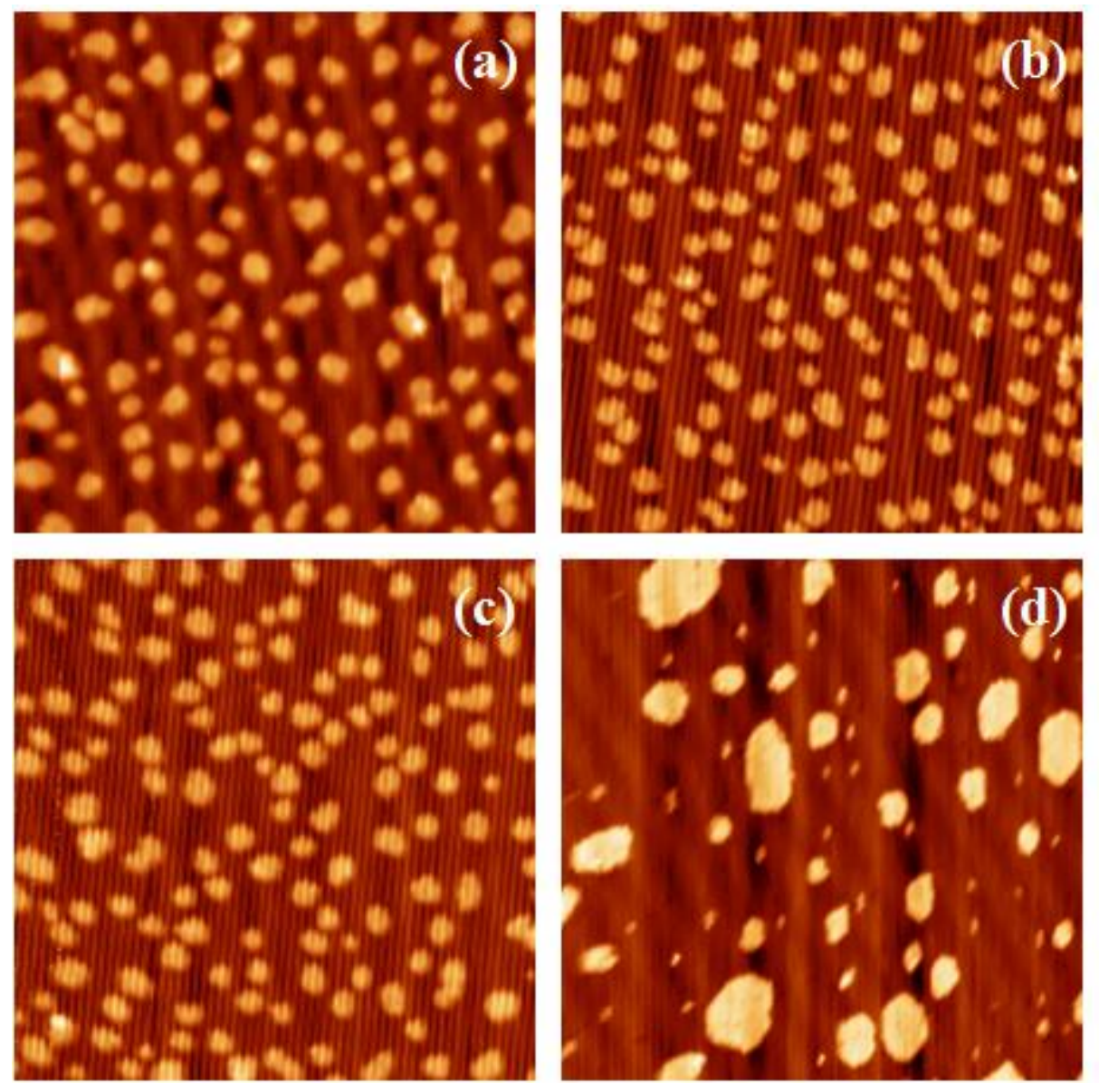

Fig. A6 

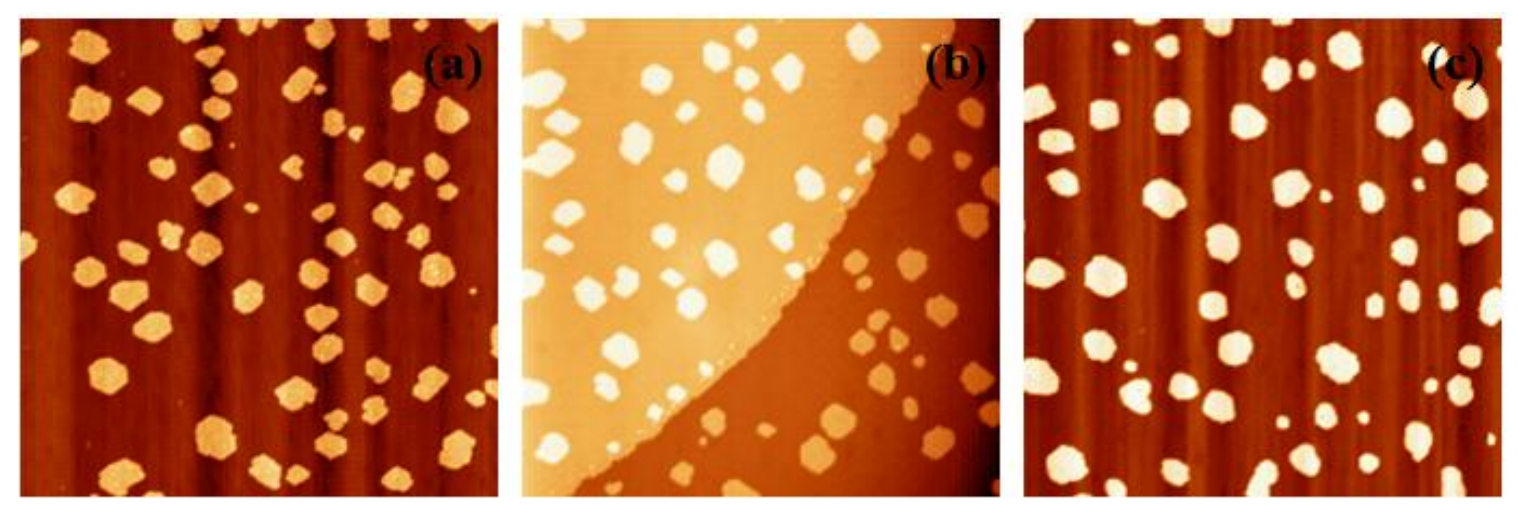

Fig. A7 


\section{GENERAL CONCLUSIONS}

The work presented in this thesis mainly focuses on the nucleation and growth of metal thin films on multimetallic surfaces. First, we have investigated the Ag film growth on a bulk metallic glass surface. Next, we have examined the coarsening and decay of bilayer $\mathrm{Ag}$ islands on $\mathrm{NiAl}(110)$ surface. Third, we have investigated the $\mathrm{Ag}$ film growth on $\mathrm{NiAl}(110)$ surface using low-energy electron diffraction (LEED). At last, we have reported our investigation on the epitaxial growth of $\mathrm{Ni}$ on $\mathrm{NiAl}(110)$ surface. Some general conclusions can be drawn as follows.

First, Ag, a bulk-crystalline material, initially forms a disordered wetting layer up to 4-5 monolayers on $\mathrm{Zr}-\mathrm{Ni}-\mathrm{Cu}-\mathrm{Al}$ metallic glass. Above this coverage, crystalline 3D clusters grow, in parallel with the flatter regions. The cluster density increases with decreasing temperature, indicating that the conditions of island nucleation are far-from-equilibrium. Within a simple model where clusters nucleate whenever two mobile Ag adatoms meet, the temperature-dependence of cluster density yields a (reasonable) upper limit for the value of the Ag diffusion barrier on top of the Ag wetting layer of $0.32 \mathrm{eV}$. Overall, this prototypical study suggests that it is possible to grow films of a bulk-crystalline metal that adopt the amorphous character of a glassy metal substrate, if film thickness is sufficiently low.

Next, the first study of coarsening and decay of bilayer islands has been presented. The system was $\mathrm{Ag}$ on $\mathrm{NiAl}(110)$ in the temperature range from $185 \mathrm{~K}$ to $250 \mathrm{~K}$. The coarsening behavior, has some similarities to that seen in the $\operatorname{Ag}(110)$ homoepitaxial system studied by Morgenstern and co-workers.[1] At $185 \mathrm{~K}$ and $205 \mathrm{~K}$, coarsening of Ag islands follows a Smoluchowski ripening pathway. At $205 \mathrm{~K}$ and $250 \mathrm{~K}$, the terrace diffusion limited 
Ostwald ripening dominants. The experimental observed temperature for the transition from SR to OR is $205 \mathrm{~K}$. The SR exhibits anisotropic island diffusion and the OR exhibits $1 \mathrm{D}$ decay of island length while keeping the corresponding island width constant.

Third, LEED indicates that, up to about 6 BL (12 ML), the Ag film adopts the (110) structure on lattice matched $\mathrm{NiAl}(110)$ surface, supporting the previous assignment based upon island heights measured in STM.[2] Starting at 4.5 to $6 \mathrm{BL},(111)$ diffraction pattern is detected. This is also in agreement with previous STM study. Careful examinations of the LEED patterns reveal the slight difference in lattice constants between bulk Ag and bulk NiAl.

At last, we performed STM studies of Ni deposition on $\mathrm{NiAl}(110)$ in the temperature range from $200 \mathrm{~K}$ to $400 \mathrm{~K}$. Ni forms “dense” Ni(100)-like islands on $\mathrm{NiAl}(110)$ with a zigzag shaped stripe feature which is probably due to strain relief. DFT analysis provides insights into the island growth shapes, which are rationalized by the thermodynamics and kinetics of the film growth process. For thick Ni films (coverage exceeding $6 \mathrm{ML}$ ), a Ni(111)-like structure developed. Traditional MF theory is applied to analyze island density at 200 K.[3] Deviation from homogeneous nucleation behavior for island size distribution and island density reveals the presence of heterogeneous nucleation mediated by the $\mathrm{Ni}$ antisite point defects on $\mathrm{NiAl}(110)$ surface.

\section{References}

[1] K. Morgenstern, E. Laegsgaard, I. Stensgaard, F. Besenbacher, Phys Rev Lett 83/8 (1999) 1613. 
[2] B. Unal, F. Qin, Y. Han, D.J. Liu, D.P. Jing, A.R. Layson, C.J. Jenks, J.W. Evans, P.A. Thiel, Phys Rev B 76/19 (2007).

[3] Y. Han, J.W. Evans, unpublished results. 


\title{
APPENDIX I. FAR-FROM-EQUILIBRIUM FILM GROWTH ON ALLOY SURFACES: Ni AND Al ON NiAl(110)
}

\author{
A paper submitted to Physical Review Letters
}

Yong Han, Dapeng Jing, Barış Ünal, P. A. Thiel and J. W. Evans

\begin{abstract}
STM analysis reveals diverse non-equilibrium island structures formed by deposition of $\mathrm{Ni}$ and $\mathrm{Al}$ on $\mathrm{NiAl}(110)$ at around $300 \mathrm{~K}$. Epitaxial growth in this complex alloy system is described by multi-site lattice-gas modeling incorporating DFT energetics for adatoms both at adsorption sites and transition states. This approach accounts for multiple adsorption sites and diffusion paths, and accurately describes diffusion-detachment kinetics for a vast number of step edge configurations. This is key for realistic description of observed island growth shapes and imperfect alloy ordering.
\end{abstract}


Homoepitaxial growth (A on A) of metal films leads to the development of an extraordinary variety of complex far-from-equilibrium morphologies at lower temperatures $\mathrm{T}$ in systems which have simple equilibrium states $[1,2]$. Such morphologies are recognized to result from system-specific kinetic limitations of step edge or interlayer diffusion on the time-scale of film growth. Essentially the same behavior can be observed in heteroepitaxy (A on B) at least with low lattice misfit, whereas distinct strain-induced features occur for higher misfit [3]. Other studies have considered alloy overlayers and films (A + B on A or on C). Often the focus was on near-equilibrium structure assessing short-range order $[4,5]$ or complex patterns reflecting interplay between chemical interactions and strain [6]. In addition, there has been extensive interest in the competition during deposition between alloy ordering and either island growth [7-11] or kinetic roughening $[12,13]$ often in cases where strain is not significant. Unfortunately, such investigations invariably used idealized models with generic prescriptions of diffusion dynamics which cannot capture system-specific nonequilibrium behavior. This motivates development of new modeling approaches to describe, elucidate and ideally facilitate control of such complex behavior.

Rather than the single-element single-crystal substrates employed in the above studies, use of binary alloy (BC) substrates would provide new opportunities to guide the creation of thin film nanostructures with desired properties. However, analyses of film growth on binary alloys (A or B or B + C on BC) are rare [14], particularly for far-fromequilibrium growth at low $\mathrm{T}$ with effectively frozen substrate dynamics [15]. Even for latticematched overlayers, there are significant complications for alloy substrates such as distinct types of adsorption sites and diffusion pathways. This complicates description of edge diffusion and detachment which can occur for vast number of local step edge environments, 
especially for co-deposition. Thus, a realistic atomistic-level description of non-equilibrium growth in such complex alloy systems pursued here requires a more general formalism than those used for simpler systems. Multi-site lattice-gas (LG) models provide the flexibility to describe multiple adsorption sites and diffusion paths. However, precise determination of diffusion barriers controlling kinetics requires probing energetics with high-level density functional theory (DFT) for adatoms not just at adsorption sites but also at transition states for diffusion (as described below). Model behavior can then be assessed via kinetic Monte Carlo (KMC) simulation.

In this Letter, we analyze submonolayer deposition of $\mathrm{Ni}$ and $\mathrm{Al}$ on $\mathrm{NiAl}(110)$ mostly at $300 \mathrm{~K}$. Shapes of islands of $\mathrm{Ni}$ or Al deposited separately exhibit a variety of nonequilibrium growth shapes. Structures formed by sequential co-deposition exhibit a strong dependence on history (i.e., deposition order) in contrast to the perfect equilibrium alloy ordering. Realistic multi-site LG modeling is developed for this system with energetics guided by DFT to accurately describe both thermodynamics (adsorption and interaction energies) and kinetics as described above. This modeling both elucidates and predicts complex growth structures.

Experimental details of $\mathrm{NiAl}(110)$ sample preparation and of the UHV chamber are described elsewhere [15]. Ni was evaporated from a commercial source (Mantis) and Al from a homemade source. Images of film morphologies were acquired with an Omicron VTSTM. Sample orientation was checked by co-deposition of Ag which forms bilayer islands elongated in the [001] direction [15]. Film composition and purity were checked with AES and XPS. Our DFT analysis was performed using the planewave based VASP package within 
the generalized gradient approximation using the PAW approach and PBE functional (see Ref. [16] for more details).

Ni deposition at $300 \mathrm{~K}$ with flux $\mathrm{F}=3 \times 10^{-3} \mathrm{ML} / \mathrm{s}$ produces islands with a density of $4.5 \times 10^{-3} \mathrm{~nm}^{-2}$ at $\sim 0.1 \mathrm{ML}$, and a height of $\sim 0.2 \mathrm{~nm}$ (from line scans with a weak tip bias dependence) corresponding to monolayer islands. Ni islands are rather irregular and on average somewhat elongated along the NiAl [001] direction (vertically in our images). See Fig. 1(a). There appears to be some preference for diagonal step edges which is amplified by deposition for lower F at $300 \mathrm{~K}$ (not shown). In contrast, deposition of $\mathrm{Ni}$ at $400 \mathrm{~K}$ (inset) produces more geometric quasi six-sided Ni monolayer islands with diagonal and vertical [001] edges, (i.e., octagons missing horizontal edges). However, these do not correspond to equilibrium shapes. Annealing to $500 \mathrm{~K}$ produces more rounded eight-sided distorted octagonal Ni islands which should be closer to equilibrated (see below).

Al deposition at $300 \mathrm{~K}$ with $\mathrm{F}=7 \times 10^{-3} \mathrm{ML} / \mathrm{s}$ produces islands with a density of $0.7 \times 10^{-2} \mathrm{~nm}^{-2}$ at $\sim 0.1$ ML likely with some contribution from heterogeneous nucleation. The measured island height of $\sim 0.2 \mathrm{~nm}(-2 \mathrm{~V})$ or $\sim 0.3 \mathrm{~nm}(+2 \mathrm{~V})$ has a strong tip bias dependence, but corresponds to monolayer islands. Al islands have distinct shapes from $\mathrm{Ni}$ at $300 \mathrm{~K}$, still irregular with some propensity for [001] steps. See Fig. 1(b).

For sequential co-deposition first of $0.20 \mathrm{ML} \mathrm{Al}$ at $\mathrm{F}=7 \times 10^{-3} \mathrm{ML} / \mathrm{s}$ and then of $0.24 \mathrm{ML} \mathrm{Ni}$ at $\mathrm{F}=3 \times 10^{-3} \mathrm{ML} / \mathrm{s}$, one obtains irregular monolayer islands. For these mixed $\mathrm{Al}-\mathrm{Ni}$ islands, the height is also bias-dependent. For $\mathrm{a}+2 \mathrm{~V}$ bias, the $\sim 0.3 \mathrm{~nm}$ high $\mathrm{Al}$ core contrasts the $\sim 0.2 \mathrm{~nm}$ high Ni ring. See Fig. 1(c). For a $-2 \mathrm{~V}$ bias, both the Al core and Ni ring are $\sim 0.2 \mathrm{~nm}$ high (no contrast). Annealing to around $460-530 \mathrm{~K}$ retains irregular islands (not 6-sided geometric islands as for $\mathrm{Ni}$ ). 
In contrast, for sequential co-deposition first of $0.15 \mathrm{ML} \mathrm{Ni}$ at $\mathrm{F}=1.7 \times 10^{-3} \mathrm{ML} / \mathrm{s}$ and then of $0.22 \mathrm{ML} \mathrm{Al}$ at $\mathrm{F}=3 \times 10^{-3} \mathrm{ML} / \mathrm{s}$, one obtains a bilayer structure with $0.06 \pm 0.01$ ML of $\mathrm{Al}$ in the 2 nd layer in the form of multiple small islands (most $\mathrm{Al}$ is in the 1st layer). See Fig. 1(d) where a $-2 \mathrm{~V}$ bias is chosen to highlight the 2 nd layer islands. A $+2 \mathrm{~V}$ bias image also reveals a $\mathrm{Ni}$ core $\mathrm{Al}$ ring structure in the 1st layer. Annealing above $500 \mathrm{~K}$ aggregates 2nd layer Al into larger islands. The measured 2nd layer coverage is consistent with a value of $0.052 \mathrm{ML}$ corresponding to all $\mathrm{Al}$ deposited on top of these growing firstlayer $\mathrm{Ni}-\mathrm{Al}$ composite structures staying in the upper layer (i.e., no interlayer transport).

To develop a detailed atomistic-level understanding of these observations, we first consider the binding and diffusion of isolated adatoms on $\mathrm{NiAl}(110)$. Both $\mathrm{Ni}$ and $\mathrm{Al}$ prefer the Ni short bridge (Ni-br), although Ni resides at the Al short bridge (Al-br) in an equilibrated alloy film! See Fig. 2(a). Ni makes diagonal hops between neighboring Ni-br and Al-br sites, diffusing with an isotropic barrier of $\mathrm{E}_{\mathrm{d}}=0.40 \mathrm{eV}$. In contrast, $\mathrm{Al}$ diffuses preferentially in the [001] direction between Ni-br sites over on-top Ni sites with a barrier of $\mathrm{E}_{\mathrm{d}}=0.30 \mathrm{eV}$. Al can also hop in the [110] direction over Al-br sites with a barrier of $\mathrm{E}_{\mathrm{d}}=$ 0.51 eV. See Fig. 2(a) for DFT adsorption energies at Ni-br and Al-br sites and at transition states (TS). For aggregated adatoms within "dense" islands, population of the Al-br site (in addition to the Ni-br site) by both $\mathrm{Ni}$ and $\mathrm{Al}$ is stabilized by adatom interactions (see below). Also, Al diffusion along island edges occurs predominantly via diagonal hops [dashed yellow arrows in Fig. 1(a)] like Ni, a different pathway than for isolated $\mathrm{Al}$ [solid yellow arrows in Fig. 1(a)]. These features necessitate multi-site LG modeling.

To prescribe adlayer thermodynamics, we determine from DFT the pair interactions between various types of adatoms at both types of adsorption sites for various separations. 
See Figs. 2(c) and 2(d). Correct treatment of non-equilibrium growth kinetics also requires an accurate description of diffusion barriers for the various pathways above and for all local island edge configurations. To this end, we also determine pair interactions with one adatom at a TS and another at nearby adsorption sites. See Table 1. Then, hopping barriers are determined from $E_{a c t}=E_{T S}-E_{\text {init }}$, where $E_{\text {init }}\left(E_{T S}\right)$ is the total energy in the initial (transition) state. Both energies are obtained from a sum of the relevant adsorption energy and all pair interaction energies. Detailed-balance is automatically satisfied. One caveat for Al hopping is that the true TS could be an initial or final unstable Al-br site. However, the above algorithm is still valid. If the initial state is an unstable Al-br site with energy above the Ni-top or final Ni-br site, then $\mathrm{E}_{\mathrm{act}}=0$ and the Al hops effectively instantaneously from that site (with rate equal to the prefactor). The Arrhenius hop rates with suitable prefactors provide input to KMC simulation of an atomistic multi-site LG model with adatoms at both Ni-br and Al-br sites. Deposition and hopping are implemented with the appropriate relative rates. This formulation provides a powerful general strategy for modeling kinetics in complex alloy thin film systems.

We could but do not include many-body interactions, as pairwise contributions likely dominate thermodynamics and kinetics. We also neglect strain effects. Actually, dense Ni islands occupying both $\mathrm{Ni}$-br and Al-br sites (see below) have little lateral strain, the $\mathrm{Ni}$ atom separation matching that for $\mathrm{Ni}(100)$ to within $0.2 \%$. Mixed stoichiometric $\mathrm{Ni}+\mathrm{Al}$ adlayers with alloy ordering are also unstrained. Dense $\mathrm{Al}$ islands do have some compressive strain. Despite some simplifications, our treatment is far more detailed and realistic than previous studies of far-from-equilibrium alloy overlayer kinetics. 
First, we describe consequences of our model energetics for adlayer thermodynamics. The energy per adatom is lower for complete dense adlayers of $\mathrm{Ni}$ or of $\mathrm{Al}$ populating both Ni-br and Al-br sites than for complete dilute adlayers populating just Ni-br or just Al-br sites: -5.40 vs. -4.70 (Ni-br) or $-4.65 \mathrm{eV}$ (Al-br) for Ni; -4.78 vs. -3.82 (Ni-br) or $-3.36 \mathrm{eV}$ (Al-br) for Al. This in part reflects strong diagonal nearest-neighbor (NN) adatom interactions. For mixed $\mathrm{Ni}+\mathrm{Al}$ adlayers, perfect alloy ordering with $\mathrm{Ni}(\mathrm{Al})$ on the correct Al-br (Ni-br) sites has the lowest energy of $-11.45 \mathrm{eV}$ per Ni-Al pair. One obtains $-10.37 \mathrm{eV}$ per pair for perfect alloy ordering on the wrong sites, and $-10.16 \mathrm{eV}$ for phase-separated dense $\mathrm{Ni}$ and $\mathrm{Al}$ islands. Regarding step energies, for $\mathrm{Ni}$ islands and ordered $\mathrm{Ni}+\mathrm{Al}$ islands, energies for diagonal steps are lower than other orientations. This preference for diagonal steps derives from strong diagonal $\mathrm{NN} \mathrm{Ni-Ni}$ and $\mathrm{Ni}-\mathrm{Al}$ interactions relative to other interactions. (Note that one diagonal bond is broken per atom for diagonal steps versus two for horizontal or vertical steps.) For Al islands, energies for [001]-oriented and diagonal steps are lowest (higher energies for horizontal steps reflecting relatively strong vertical NN Al-Al interactions).

Now we turn to our central focus, the kinetics of island formation. For nucleation and growth of Ni islands mediated by almost isotropic terrace diffusion, the most stable dimer is a diagonal NN pair. The "bare" interaction strength is $0.34 \mathrm{eV}$, but since one Ni is at an Al-br site with a lower adsorption energy by $0.15 \mathrm{eV}$, the effective binding energy (relative to separated $\mathrm{Ni}$ on $\mathrm{Ni}$-br sites) is $\mathrm{E}_{\mathrm{b}}=0.19 \mathrm{eV}$. This implies island formation is marginally reversible at $300 \mathrm{~K}$ [2]. Simulation results from our atomistic model with a prefactor for $\mathrm{Ni}$ hopping of $v=5 \times 10^{11} / \mathrm{s}$ recover island shapes, as well as matching the observed island density. See Fig. 3(a). 
Edge diffusion is active along diagonal steps at $300 \mathrm{~K}$ with barrier $\mathrm{E}_{\mathrm{e}} \sim 0.35 \mathrm{eV}$, but not along horizontal or vertical steps $\left(\mathrm{E}_{\mathrm{e}} \sim 0.85 \mathrm{eV}\right)$, so island growth shapes have a preference for diagonal steps. This is analogous to square island growth shapes in metal (100) homoepitaxy with rapid diffusion along close-packed steps and limited diffusion along kinked steps [2]. Why the tendency for vertical elongation of Ni islands? Our DFT energetics indicate an anisotropy in corner rounding from diagonal to horizontal vs. vertical steps, the former being easier. Thus, edge atoms are more easily fed to the top and bottom of the growing island. For deposition at $\sim 400 \mathrm{~K}$, diffusion along horizontal and vertical steps becomes active potentially allowing such straight steps to form. However, anisotropic corner rounding causes the horizontal steps to grow faster (and thus grow out) resulting in the growth of 6-sided islands observed by STM. See Fig. 3(b). Annealing above $500 \mathrm{~K}$ starts to produce quasi-equilibrated 8-sided shapes. See Fig. 3(c). Nucleation and growth of Al islands is mediated by strongly anisotropic terrace diffusion. The most stable dimer is vertically aligned on Ni-br sites with a binding energy of $\mathrm{Eb}=0.38 \mathrm{eV}$, implying effectively irreversible island formation at $300 \mathrm{~K}$ [2]. Note that the stronger "bare" interaction for a vertical dimer on Al-br sites is offset by a large adsorption site penalty, so this dimer is not stable. Simulation recovers experimental island shapes. See Fig. 3(d). The island density is a bit low even with a low prefactor $v=2.5 \times 10^{11} / \mathrm{s}$ for Al hopping, likely due to some heterogeneous nucleation. With regard to island growth, it is appropriate to note that if one allowed only the diffusion pathway for isolated Al adatoms (hopping through on-top Ni TS), edge diffusion would be effectively inoperative and simulations would produce very irregular or fractal islands. Edge diffusion via diagonal hops along horizontal edges is active with a 
barrier of $\mathrm{E}_{\mathrm{e}} \sim 0.5 \mathrm{eV}$ facilitating formation of straight vertical edges. Thus our general multisite multi-path diffusion LG modeling is essential.

For sequential co-deposition first of $\mathrm{Al}$ and then of $\mathrm{Ni}$, one does recover the observed monolayer island structures with an Al-core and surrounding Ni-ring. We find little intermixing at the Al-Ni interface. See Fig. 3(e). This suggests ring structures are common for sequential co-deposition [17], not rare as recently suggested [10]. For comparison, we have also performed simulations of simultaneous stoichiometric co-deposition of $\mathrm{Ni}$ and $\mathrm{Al}$. Here, one finds intermixing but poor alloy ordering under these far-from-equilibrium growth conditions. See Fig. 3(f).

Finally, for sequential co-deposition first of $\mathrm{Ni}$ and then of $\mathrm{Al}$, experiments found 2 nd layer population by $\mathrm{Al}$ and thus a very different island structure from the above co-deposition procedures. To explain this behavior, we have performed a DFT analysis revealing that adsorption for isolated $\mathrm{Al}$ on top of a dense $\mathrm{Ni}$ monolayer on $\mathrm{NiAl}(110)$ is $\geq 1 \mathrm{eV}$ stronger per adatom than directly on the substrate. This enhanced binding would strongly inhibit any downward transport of Al.

In summary, deposition of $\mathrm{Ni}$ and $\mathrm{Al}$ at $300 \mathrm{~K}$ on $\mathrm{NiAl}(110)$ produces a rich variety of far-from-equilibrium nanostructures even for stoichiometric co-deposition where the equilibrium structure exhibits perfect alloy ordering. Multi-site LG modeling incorporating DFT guided energetics for both interactions and barriers allows a realistic treatment of farfrom-equilibrium growth in these complex systems. This approach has general applicability for alloy systems.

Modeling, STM of Ni, and Al then Ni were supported by NSF Grant CHE-0809472 (YH, DJ, PT, JE); STM of Al, and Ni then Al by USDOE-BES Materials Sciences (BU). 
Computational support at NERSC was provided by the USDOE. Ames Laboratory operated for the USDOE by ISU under Contract No. DE-AC02-07CH11358.

\section{References}

[1] T. Michely, and J. Krug, Islands, Mounds, and Atoms (Springer, Berlin, 2004).

[2] J. W. Evans, P. A. Thiel, and M. C. Bartelt, Surface Science Reports 61, 1 (2006).

[3] R. Q. Hwang, and M. C. Bartelt, Chem. Rev. (Washington, D. C.) 97, 1063 (1997).

[4] H. Hartmann et al., Surf Sci 603, 1439 (2009).

[5] M. Chen et al., Science (Washington, DC, U. S.) 310, 291 (2005).

[6] G. E. Thayer et al., Phys Rev Lett 89, 036101/1 (2002).

[7] M. Kotrla, J. Krug, and P. Smilauer, Phys. Rev. B: Condens. Matter 62, 2889 (2000).

[8] T. Volkmann et al., Surf Sci 586, 157 (2005).

[9] S. Weber et al., J. Phys.: Condens. Matter 20, 265004/1 (2008).

[10] F. Dumont et al., Phys. Rev. B: Condens. Matter 77, 153404/1 (2008).

[11] M. Einax et al., Phys Rev Lett 99, 016106/1 (2007).

[12] J. R. Smith, Jr., and A. Zangwill, Phys Rev Lett 76, 2097 (1996).

[13] E. N. Oskoee, M. R. H. Khajehpour, and M. Sahimi, Phys. Rev. E: Stat,, Nonlinear, Soft Matter Phys. 69, 061606/1 (2004).

[14] J. P. Pierce, N. C. Bartelt, and K. F. McCarty, Phys Rev Lett 99, 026101 (2007).

[15] Y. Han et al., Phys Rev Lett 100, 116105/1 (2008).

[16] Y. Han et al., Phys. Rev. B: Condens. Matter 81, 115462/1 (2010).

[17] M. C. Bartelt et al., Phys Rev Lett 81, 1901 (1998). 


\section{Tables}

Table 1: Interaction energies in $\mathrm{eV}$ (attraction $>0$ ) between $\mathrm{Ni}$ and $\mathrm{Al}$ adatoms where one adatom is at an adsorption site (Ni-br sites 1, 3, ..; Al-br sites 2, 4, ..) and another is at a TS (Ni-top site t or the Ni-Al bridge site b), see Fig. 2(a).

\begin{tabular}{|l|c|c|c|c|c|}
\hline $\mathrm{Ni}(\mathrm{b})-\mathrm{Ni}(3)$ & 0.25 & $\mathrm{Al}(\mathrm{b})-\mathrm{Al}(3)$ & 0.29 & $\mathrm{Ni}(\mathrm{b})-\mathrm{Al}(3)$ & 0.71 \\
\hline $\mathrm{Ni}(\mathrm{b})-\mathrm{Ni}(5)$ & 0.14 & $\mathrm{Al}(\mathrm{b})-\mathrm{Al}(5)$ & 0.40 & $\mathrm{Ni}(\mathrm{b})-\mathrm{Al}(5)$ & 0.28 \\
\hline $\mathrm{Ni}(\mathrm{b})-\mathrm{Ni}(6)$ & 0.30 & $\mathrm{Al}(\mathrm{b})-\mathrm{Al}(6)$ & 0.45 & $\mathrm{Ni}(\mathrm{b})-\mathrm{Al}(6)$ & 0.85 \\
\hline $\mathrm{Ni}(\mathrm{b})-\mathrm{Ni}(8)$ & 0.18 & $\mathrm{Al}(\mathrm{b})-\mathrm{Al}(8)$ & 0.20 & $\mathrm{Ni}(\mathrm{b})-\mathrm{Al}(8)$ & 0.14 \\
\hline $\mathrm{Al}(\mathrm{b})-\mathrm{Ni}(3)$ & 0.62 & $\mathrm{Al}(\mathrm{t})-\mathrm{Al}(2)$ & -1.00 & $\mathrm{Al}(\mathrm{t})-\mathrm{Ni}(2)$ & -0.44 \\
\hline $\mathrm{Al}(\mathrm{b})-\mathrm{Ni}(5)$ & 0.24 & $\mathrm{Al}(\mathrm{t})-\mathrm{Al}(3)$ & -12.00 & $\mathrm{Al}(\mathrm{t})-\mathrm{Ni}(3)$ & -6.50 \\
\hline $\mathrm{Al}(\mathrm{b})-\mathrm{Ni}(6)$ & 0.73 & $\mathrm{Al}(\mathrm{t})-\mathrm{Al}(4)$ & 0.12 & $\mathrm{Al}(\mathrm{t})-\mathrm{Ni}(4)$ & 0.06 \\
\hline $\mathrm{Al}(\mathrm{b})-\mathrm{Ni}(8)$ & 0.18 & $\mathrm{Al}(\mathrm{t})-\mathrm{Al}(7)$ & 0.02 & $\mathrm{Al}(\mathrm{t})-\mathrm{Ni}(7)$ & 0.01 \\
\hline
\end{tabular}




\section{Figure captions}

Fig. 1: STM images $\left(25 \times 25 \mathrm{~nm}^{2}\right)$ for deposition at $300 \mathrm{~K}$ : (a) $0.18 \mathrm{ML}$ Ni (Inset: Ni island at $400 \mathrm{~K}$ ); (b) 0.1 ML Al; (c) 0.20 ML Al then 0.24 ML Ni (Inset: Line scan highlighting higher $\mathrm{Al}$ core and lower Ni ring); (d) 0.15 ML Ni then 0.22 ML Al (Inset: Line scan highlighting 2nd layer $\mathrm{Al}$ islands imaged as bright dots). Tip bias: $-2 \mathrm{~V}$ for $(\mathrm{a}, \mathrm{b}, \mathrm{d}) ;+2 \mathrm{~V}$ for $(\mathrm{c})$. $\mathrm{NiAl}[001]$ direction is vertical. Insets for $(\mathrm{c}, \mathrm{d})$ : Height (width) in units of $\AA$ (nm).

Fig. 2: (a) Ni-br and Al-br adsorption site and TS binding energy magnitudes in $\mathrm{eV}$ for isolated $\mathrm{Ni}(\mathrm{Al})$ on $\mathrm{NiAl}(110)$; diffusion paths (solid green arrows for $\mathrm{Ni}$, yellow arrows for Al) are also indicated; sites $1,3, \ldots=\mathrm{Ni}$-br; $2,4, \ldots=\mathrm{Al}-\mathrm{br} ; \mathrm{t}=\mathrm{Ni}$-top; $\mathrm{b}=$ $\mathrm{Ni}$-Al bridge. (b) Ni diffusion path along a diagonal step segment of a dense Ni island (green): Lines indicate dominant $\mathrm{Ni}-\mathrm{Ni}$ interactions at adsorption sites and TS [values from (c) and Table 1]. Interaction energies in $\mathrm{eV}$ between $\mathrm{Ni}$ and $\mathrm{Al}$ adatoms at $\mathrm{Ni}$-br and Al-br adsorption sites: (c) Ni-Ni (Al-Al); (d) Ni-Al. Dashed rectangle in (d) is $\mathrm{NiAl(110)}$ surface unit cell with experimental lattice constants labeled. Dots denote adatoms or sites. Positive interactions are attractions.

Fig. 3: Simulated island configurations: (a) $0.12 \mathrm{ML}$ Ni at $300 \mathrm{~K}(26 \times 26 \mathrm{~nm} 2)$; (b) Ni at 400 $\mathrm{K}$ ( 8330 atoms); (c) Ni at $600 \mathrm{~K}(\sim 1710$ atoms); (d) $0.07 \mathrm{ML} \mathrm{Al} \mathrm{at} 300 \mathrm{~K}(24 \times 24$ $\mathrm{nm}^{2}$ ); (e) 0.01 ML Al then 0.10 ML Ni at $300 \mathrm{~K}$; (f) 0.02 ML Al and 0.02 ML Ni simultaneous co-deposition at $300 \mathrm{~K}$. 
Figures

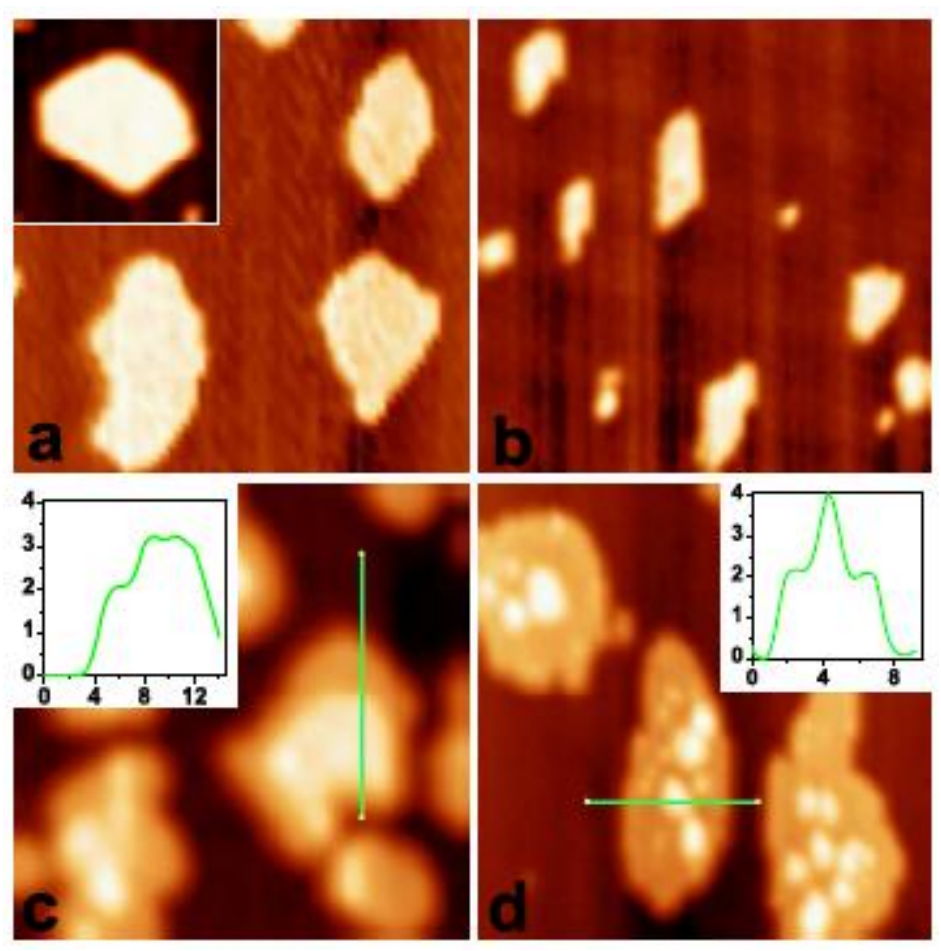

Fig. 1 


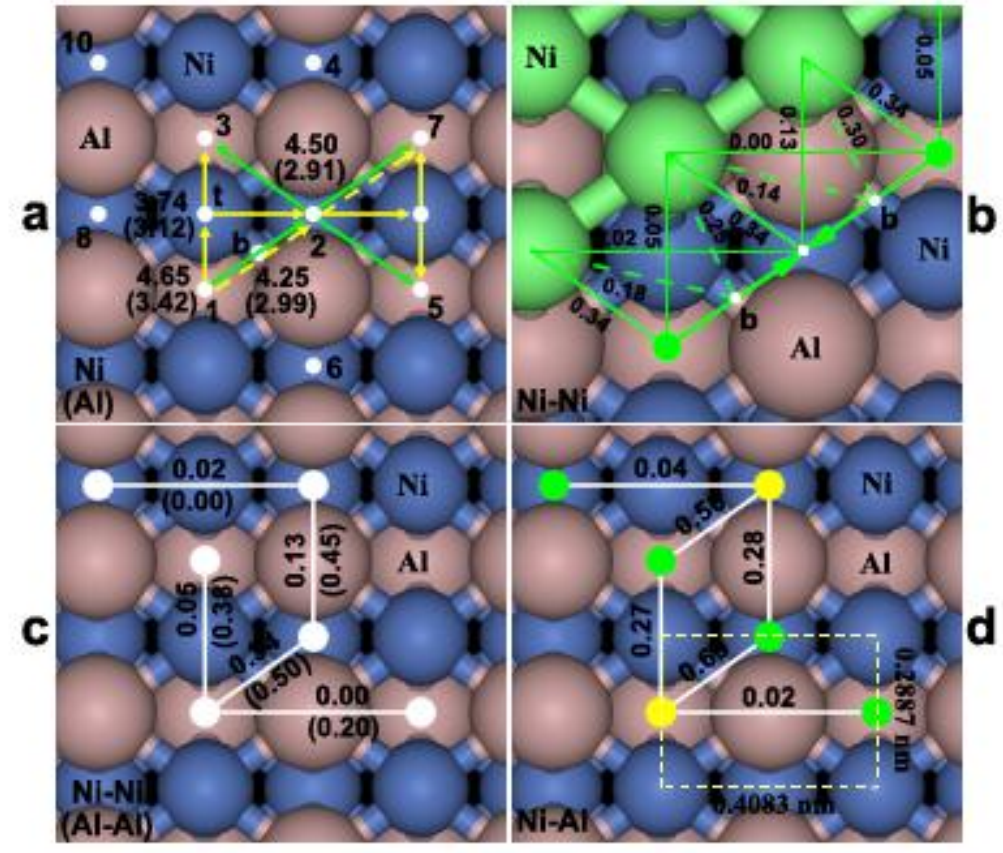

Fig. 2 

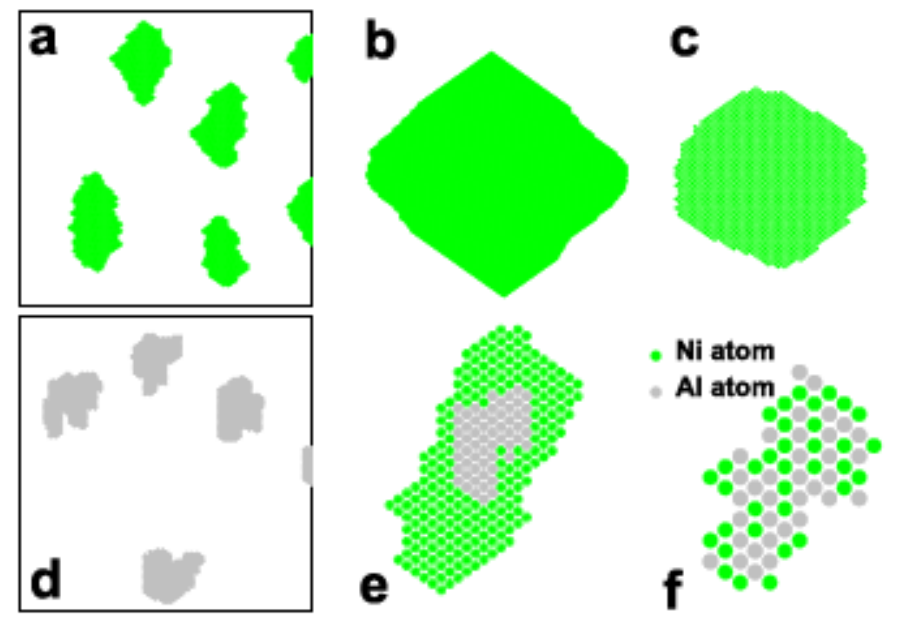

Fig. 3 


\title{
APPENDIX II. FORMATION AND COARSENING OF Ag(110) BILAYER ISLANDS ON NiAl(110): STM ANALYSIS AND ATOMISTIC LATTICE-GAS MODELING
}

\author{
A paper published in Physical Review B
}

\begin{abstract}
Yong Han, Barış Ünal, Dapeng Jing, Feili Qin, C. J. Jenks, Da-Jiang Liu, P. A. Thiel and J. W. Evans
\end{abstract}

\begin{abstract}
Scanning tunneling microscopy analysis of the initial stages of film growth during deposition of $\mathrm{Ag}$ on $\mathrm{NiAl}(110)$ reveals facile formation of bilayer $\mathrm{Ag}(110)$ islands at temperatures of $130 \mathrm{~K}$ and above. Annealing subsequent to deposition at $130 \mathrm{~K}$ induces coarsening of the bilayer island distribution. The thermodynamic driving force for bilayer island formation reflects a lower relative surface energy for films of even layer thicknesses. This feature derives from quantum size effects due to electron confinement in the Ag film. The kinetics of island formation and relaxation is controlled by terrace and edge-diffusion barriers, detachment barriers, interlayer diffusion barriers, and layer-dependent adsorption and interaction energies. These key energies are determined from density-functional theory analysis and incorporated into an atomistic lattice-gas model for homogeneous island formation, where specification of the adatom hop rates is consistent with detailed balance. Model analysis via kinetic Monte Carlo simulation elucidates the role of strongly anisotropic interactions in development during deposition of elongated island growth shapes and also in facilitating upward mass transport needed for bilayer island formation. The model succeeds
\end{abstract}


in recovering island densities at lower temperatures but experimental densities exceed model predictions at higher temperatures plausibly due to heterogeneous nucleation at surface defects. The same model successfully describes postdeposition coarsening of small islands grown at $130 \mathrm{~K}$.

\section{Introduction}

Traditionally, epitaxial film growth has been classified into three "quasiequilibrium" growth modes [1]. Frank-van der Merwe (FM) growth, i.e., layer-by-layer growth, typically occurs for overlayers with low surface free energy relative to the substrate and with low strain. In contrast, often three-dimensional (3D) islands form either during the initial stages of heteroepitaxy for Volmer-Weber growth or after development of a wetting layer for Stranski-Krastanov growth. Such 3D islands are expected for high surface-energy adsorbates. They are also expected for high interface energies often associated with the buildup of strain in the growing film.

However, growth modes quite different from those above are sometimes observed for metal-on-metal film growth. These include sandwichlike growth where atoms from a low surface-energy substrate climb on top of the overlayer. [2-4] Other possibilities of more relevance in this study include the development of bilayer islands on single-element metal substrates [4-8] and on alloy substrates. [9-11] The development of flat-topped or mesalike multilayer islands has also been observed on single-element metal substrates [12-16] and on alloys. [17,18] Formation of such morphologies can occur even for metal adsorbates with low surface energies such as $\mathrm{Ag}$ and $\mathrm{Pb}$ where one might expect FM growth. The thermodynamic driving force for growth of heightselected, mesalike multilayer metal islands 
is often associated with quantum size effects (QSE): Electron confinement within the film produces a height-dependent surface free energy so that islands with different thicknesses have different thermodynamic stabilities. Furthermore, oscillatory thermodynamic stability of a metal film upon varying thickness reflects a matching condition: The half Fermi wavelength $\lambda_{\mathrm{F} / 2}$ should be commensurate with the film thickness which is some multiple of the interlayer spacing d. [19-21] For a $\operatorname{Ag}(110)$ film, $d=0.1439 \mathrm{~nm}$ and $\lambda_{\mathrm{F}}=0.5206 \mathrm{~nm}$ at zero temperature ( $0 \mathrm{~K})$. [21] Thus, $\lambda_{\mathrm{F} / 2}$ corresponds to $90 \%$ of a bilayer film thickness of $2 \mathrm{~d}$. This indicates that $\operatorname{Ag}(110)$ films should display a primary stability oscillation with a period of two layers, in agreement with the density-functional theory (DFT) energetics. [20,21]

Formation of bilayer islands could reflect QSE. However, it is also possible that their formation primarily reflects stronger adatom adsorption and/or interaction on top of a monolayer and thicker films relative to the substrate, together with kinetic inhibition of the nucleation of 3rd and higher layers. Note also that since bilayer and 3D multilayer island formation involve upward mass transport, these processes are likely to be kinetically hindered at lower temperatures. In fact, there is little detailed analysis of the kinetics in these systems. Experimental examples where bilayer formation is inhibited (but assisted by substrate steps) exist for $\mathrm{Fe}$ on $\mathrm{Ag}(100)$, [14] and $\mathrm{Co}$ on $\mathrm{Cu}(111)$. [7] Some modeling of kinetics exists for the formation of bilayer $\mathrm{Co}$ islands on $\mathrm{Cu}(100)$, [22] and multilayer $\mathrm{Pb}$ islands on $\mathrm{Si}(111)$. [23-26] Our goal here for the $\mathrm{Ag} / \mathrm{NiAl}(110)$ system is to develop a detailed and realistic atomistic model to describe the complete island formation process, thereby elucidating the facile formation of bilayer islands even at low temperatures.

There is also interest in postdeposition stability versus coarsening of distributions of separated islands. There have been extensive studies of coarsening and decay for 3D islands 
in heteroepitaxial systems, [27] and more recently for 2D islands in homoepitaxial systems. $[28,29]$ In these systems, the thermodynamic driving force for coarsening is a reduction in the free energy associated with island periphery, so evolution is just driven by differences in effective island radii. The presence of QSE (Ref. 30) or partial bilayer island formation could produce distinct behavior. Thus, we are interested in quantifying coarsening in the $\mathrm{Ag} / \mathrm{NiAl}(110)$ system and in assessing whether behavior is also captured by the above mentioned atomistic model.

In this paper, we analyze both the formation and coarsening of bilayer Ag islands on $\mathrm{NiAl}(110)$. In Sec. 2, we briefly summarize key experimental procedures and observations, as well as describing computational procedures for our DFT calculations of energetics. Then, in Sec. 3, our scanning tunneling microscopy (STM) observations are presented for the nucleation and growth of bilayer $\mathrm{Ag}$ islands on $\mathrm{NiAl}(110)$ for deposition at $130 \mathrm{~K}$ and above. In addition, we analyze postdeposition coarsening (during annealing) of small bilayer islands formed at $130 \mathrm{~K}$. DFT results for supported Ag films are presented in Sec. 4 for adsorption energies (reflecting QSE), interaction energies, and key diffusion barriers. In Sec. 5, our atomistic model is described for this system which incorporates the above DFT energetics. We present results from kinetic Monte Carlo (KMC) simulations in Sec. 6. The model is shown to successfully describe not just facile bilayer island formation at low $\mathrm{T}$ but also postdeposition coarsening. Sec. 7 provides further discussion and summarizes our findings.

\section{Experimental details, computational methods, and island structure}

Details of our vacuum chamber setup, $\mathrm{NiAl}$ sample preparation, Ag deposition procedure, and STM analysis are described in Refs. [10,11]. STM images reveal that our 
sample preparation procedures can produce a $\mathrm{NiAl}(110)$ surface with broad terraces up to 1 $\mu \mathrm{m}$ wide. Thus, all images shown are for a single substrate terrace (although different terraces are imaged for growth studies at different $\mathrm{T}$ ). The substrate crystallographic direction is described in the captions of Figs. 1 and 2, but for all subsequent STM and KMC images, the [001] direction is always in the vertical direction on the page. In our studies of island formation, we typically deposit about 0.4 monolayers (MLs) or 0.2 bilayers (BLs) of $\mathrm{Ag}$ on $\mathrm{NiAl}(110)$ at an estimated flux of $\mathrm{F}=3.3 \times 10^{-3} \mathrm{BL} / \mathrm{s}$. One bilayer is defined as two monolayers of a perfect $\mathrm{Ag}(110)$ film.

DFT calculations are performed using the plane-wave-based Vienna ab initio simulation package (VASP). [31] We use the Perdew-Burke-Ernzerhof form of the generalized gradient approximation. [32] The electron-ion interactions are described by the projector augmented-wave approach. [33] The converged magnitude of the forces on all relaxed atoms is always less than $0.1 \mathrm{eV} / \mathrm{nm}$. To prevent spurious interactions between adjacent replicas of the thin-film system, we use a vacuum layer that is $15 \AA$ thick in the direction perpendicular to the surface. The optimized lattice constants are $\mathrm{a}_{\mathrm{Ag}}=0.4166 \mathrm{~nm}$ for $\mathrm{Ag}$, and $\mathrm{a}_{\mathrm{NiAl}}=0.2896 \mathrm{~nm}$ for $\mathrm{NiAl}$, to be compared with the experimental values $0.4086 \mathrm{~nm}$ and $0.2887 \mathrm{~nm}$, respectively. Diffusion paths and barriers are determined via the climbing nudged elastic band (cNEB) method. [34] These DFT energetics will be incorporated into an atomistic lattice-gas model for epitaxial growth of $\mathrm{Ag}(110)$ films described in Sec. 5. Model behavior is analyzed by KMC simulation.

In our model development, we assume that $\mathrm{Ag}$ islands on $\mathrm{NiAl}(110)$ have an fcc(110) structure (see Appendix A), and will find a preference to form bilayer rather than monolayer islands. One factor favoring such epitaxial growth is the almost perfect match between the 
surface unit cells for $\mathrm{NiAl}(110)$ and $\mathrm{Ag}(110)$. [10,11] This feature would also preclude lateral mismatch strain as a source of the deviation from monolayer island growth. Evidence supporting the fcc(110) bilayer structure came from agreement between the experimentally measured height of $0.32 \mathrm{~nm}$ and DFT results. [10] Furthermore, a slight periodic ripple in the height of islands was also seen in DFT analysis of relaxed fcc(110) bilayer structures. [10] Deviation from fcc(110) bilayer-by-bilayer growth is observed for much thicker films, as seen in other Ag heteroepitaxy systems due to a preference for the lower surface-energy $\operatorname{Ag}(111)$ structure. However, this behavior is not relevant for the initial stages of growth considered here.

Next, additional evidence for the bilayer fcc(110) island structure is discussed. Using DFT, we have searched for and found alternative low-energy film structures (described in Appendix A as double chain and square hex). However, none of the stable monolayer or multilayer versions of these structures is close to matching the experimentally observed island height.

Finally, there is also compelling experimental evidence from low-energy electron diffraction (LEED) analysis. We have compared the LEED pattern for the clean substrate with that for a 3.0 ML film deposited at $300 \mathrm{~K}$ (for which the substrate is completely covered by a Ag film of thickness $0.32 \mathrm{~nm}$ and $50 \%$ of that film is covered by rectangular Ag islands with step height $0.29 \mathrm{~nm}$ ). One observes essentially identical rectangular arrangements of diffraction spots for both the clean substrate and the 3.0 ML film for all electron energies examined between 100 and $250 \mathrm{eV}$. The aspect ratio of the rectangular Brillouin zone is 1.415 in both cases and the reciprocal space dimensions agree to within $0.4 \%$. It should be emphasized that for these energies, the mean-free path for electrons in Ag is short, as low as 
$0.65 \mathrm{~nm}$ and no higher than $0.8 \mathrm{~nm}$. [35] Thus, essentially all of the LEED intensity comes from diffraction from the Ag film, not the substrate, so the above observations provide compelling evidence for the fcc(110) structure of the $\mathrm{Ag}$ film. We also note that upon varying the electron energy, adjacent spots display out-of-phase oscillation, also as expected for a fcc structure. [36,37]

\section{STM analysis of bilayer island evolution}

Fig. 1 shows rectangular islands formed by depositing $\sim 0.2 \mathrm{BL}$ of $\mathrm{Ag}$ on $\mathrm{NiAl}(110)$ at temperatures between $\mathrm{T}=127$ and $300 \mathrm{~K}$ with $\mathrm{F}=3.3 \times 10^{-3} \mathrm{BL} / \mathrm{s}$. Their height is always $0.32 \mathrm{~nm}$, a value consistent with a $\operatorname{Ag}(110)$ bilayer structure. Island densities Nisl are shown in Table I for various T.

If an island density is determined from images containing a total of $\mathrm{M}$ islands, then there is an uncertainty in density associated with finite sample size. This follows from the uncertainty (standard deviation), $\delta \mathrm{M}$, in the number of islands, where $(\delta \mathrm{M})^{2} \approx \mathrm{CM}$, an expression based on exact fluctuation-correlation relations $[38,39]$ which relate $\mathrm{C}$ to island pair correlations. Our simulations indicate that $\mathrm{C} \approx 1 / 2$ for lower $\mathrm{T}$, although one might conservatively choose $\mathrm{C} \approx 2-4$, say, to account for the effects of possible heterogeneity of the substrate. Thus, in Table I, we also report the total number, M, of islands counted at each T

allowing determination of the percentage uncertainty in the island density from $100(\mathrm{C} / \mathrm{M})^{1 / 2}$. However, there is another source of uncertainty associated with our percolation-type definition of islands (used in the simulations), wherein two barely connected islands should be counted as one. From the STM images, it is not clear whether islands are connected 
particularly for higher densities of smaller islands. This introduces an additional uncertainty of perhaps $\pm 10 \%$ at $127 \mathrm{~K}$ but significantly lower at higher $\mathrm{T}$.

The variation in island density with $\mathrm{T}$ is described for lower $\mathrm{T}$ by $\mathrm{N}_{\mathrm{isl}} \propto \mathrm{e}^{-\beta \mathrm{E}}$ with an Arrhenius energy of $\mathrm{E}=0.08-0.09 \mathrm{eV}$. Here, $\beta \equiv 1 /\left(\mathrm{k}_{\mathrm{B}} \mathrm{T}\right)$, and $\mathrm{k}_{\mathrm{B}}$ is the Boltzmann constant. This behavior might be interpreted by a traditional mean-field rate equation (RE) analysis $[38,40]$ assuming effectively irreversible homogeneous nucleation and growth of islands (i.e., critical size $i=1$ ) with near-isotropic terrace diffusion (which we will show is appropriate here). Then, the observed Arrhenius behavior implies an isotropic diffusion barrier of $E_{d}=3 E=0.24-0.27 \mathrm{eV}$ for $\mathrm{Ag}$ on $\mathrm{NiAl}(110)$. However, this treatment is overly simplistic.

To scrutinize the above nucleation analysis, one can utilize previous simulation results for irreversible formation of compact islands with isotropic diffusion. [38,41] Using $\mathrm{E}_{\mathrm{d}}=0.27 \mathrm{eV}$, a prefactor of $v=10^{13} / \mathrm{s}$ and the experimental $\mathrm{F}$ yields $\mathrm{N}_{\mathrm{is}}=4.1 \times 10^{-2} \mathrm{~nm}^{-2}$ at $140 \mathrm{~K}$ compared with $1.8 \times 10^{-2} \mathrm{~nm}^{-2}$ from experiment. This discrepancy (neglected previously ${ }^{11}$ ) reflects some reversibility in island formation at $140 \mathrm{~K}$. This is confirmed by analysis of the "crossover variable" $[38,42]$

$$
Y=\frac{v}{F} e^{-\beta\left(E_{d}+1.5 E_{b}\right)}
$$

where $v$ is the attempt frequency, $\mathrm{E}_{\mathrm{d}}$ is the diffusion barrier (as above), and $\mathrm{E}_{\mathrm{b}}$ is the bond strength controlling dimer stability. Y reflects the ratio of dimer dissociation to island growth rates and Y exceeding $~ 10$ corresponds to reversible island formation. From this analysis, one concludes that island formation is reversible at $140 \mathrm{~K}$ for $\mathrm{E}_{\mathrm{b}}<0.1 \mathrm{eV}$ which we show applies in this system. 
Another complication for this system is that the decrease in island density with increasing T up to $300 \mathrm{~K}$ is significantly slower than would be expected given the onset of reversibility below $150 \mathrm{~K}$. Note however that the substrate is a binary alloy surface which in general will display some deviation of near surface from bulk composition leading to point defects associated with substitutional exchange or replacement. Indeed, point defects have been observed by STM on the $\operatorname{NiAl}(110)$ surface in our own work and that of other groups. $[43,44]$ They were associated with replacement of Al with Ni to create a Ni-rich surface and with subsurface interstitials. All groups report that these defects are visible for stronger bias conditions but not for lower bias where STM images show surface states as waves. Point defects present similar STM images on other binary alloy surfaces. [45] More complex extended defects such as dislocations and antiphase boundaries were also observed on $\mathrm{NiAl}(110)$ surfaces and proposed to play a key role in Al film growth at high T. [46] Suppose that the above point or extended defects form "strong traps" for nucleation. Then, if the effective defect density is low relative to the density of homogeneously nucleated Ag islands at around $140 \mathrm{~K}$, these defects should not significantly affect island formation at lower T. However, they could impact behavior for higher T. Specifically, an increasing fraction of islands could be nucleated at defect traps for higher $\mathrm{T}$, boosting island density above that for homogeneous nucleation. See Appendices B and C for further analysis. As an aside, another possibility is that buried extended defects might cause "slight perturbations" of the potential-energy surface (PES) seen by diffusing adatoms. These perturbations may not have a significant effect at high $\mathrm{T}$ but could impact homogeneous nucleation at low $\mathrm{T}$. 
As noted above, the Ag bilayer islands formed during deposition on $\mathrm{NiAl}(110)$ have rectangular shapes which are elongated in the [001] crystallographic direction of the $\mathrm{NiAl}(110)$ substrate. Furthermore, the aspect ratio of the islands increases with increasing deposition temperature for the entire range probed in these experiments (up to $300 \mathrm{~K}$ ). In contrast, for $\mathrm{Ag} / \mathrm{Ag}(110)$ homoepitaxy, [38] one finds that rectangular single-layer high islands form during deposition. Their aspect ratio increases with increasing deposition temperature up to about $200 \mathrm{~K}$ but then decreases strongly for temperatures around 220-250 K (depending on F). Presumably the bilayer structure of Ag islands on NiAl(110) "locks in" the nonequilibrium growth shapes. In contrast, the monolayer islands on $\mathrm{Ag}(110)$ are much more dynamic (as confirmed by our simulation studies described below), and thus able to achieve their equilibrium shapes below $300 \mathrm{~K}$.

As an aside, it is appropriate to mention that atomic chains of noble metals ( $\mathrm{Au}, \mathrm{Ag})$ have been assembled on the $\mathrm{NiAl}(110)$ substrate using the STM tip following deposition at very low T. [47-49] Typically, these chains are oriented in the [001] direction which is the natural elongation direction of our self-assembled islands. The correspondence of the separation of adjacent atoms in a chain of this orientation with that in the bulk was presumed to facilitate chain formation. [47] It is however also possible to assemble chains in the orthogonal direction. [48] Rather than troughs on the surface, [49] we would argue that stronger interactions between adatoms aid assembly and stabilization of [001]-oriented chains.

Finally, our analysis of the coarsening of bilayer $\mathrm{Ag}$ island distributions is described. Since bilayer islands are relatively stable (cf. above), we enhance the propensity for coarsening in two ways. First, we deposit Ag at low T=130 K and with "high" $\mathrm{F}=2.8 \times 10^{-2}$ 
$\mathrm{BL} / \mathrm{s}$ to increase the density of islands and thus to reduce their mean size (for fixed coverage). The smaller size implies higher effective curvature of island edges and a higher spread of curvatures, thus producing a stronger driving force for coarsening. In addition, deposition for sufficiently low $\mathrm{T}$ and high $\mathrm{F}$ could lead to formation of monolayer or incomplete bilayer islands, which should be more susceptible to coarsening. Second, we ramp up the temperature after deposition (cf. Fig. 2) in order to enhance the thermally activated coarsening process.

Results shown in Fig. 2 do indeed reveal significant coarsening already when the temperature has climbed to $175 \mathrm{~K}$ and extensive coarsening above $200 \mathrm{~K}$. Island shapes become rectangular with increasing aspect ratio above $200 \mathrm{~K}$. This presumably reflects a transition from low-T growth shapes towards higher-T equilibrium shapes. These quasiequilibrium shapes are not as elongated as the growth shapes from direct deposition at around $300 \mathrm{~K}$.

\section{DFT analysis of key energetics}

\subsection{Interaction energies}

The dependence on thickness L (measured in layers), of the average energy per Ag atom in the supported $\operatorname{Ag}(110)$ film, $E_{L}^{A g}$, controls the thermodynamic "growth" mode. However, to elucidate nonequilibrium growth behavior, we consider additional quantities including the $\mathrm{L}$ dependence of the adsorption energy of an isolated $\mathrm{Ag}$ adatom, $E_{L}^{\text {ads }}$, upon a film of $\mathrm{L}^{-1}$ layers, and the layer dependence of lateral interactions within the film. 
For low-T growth with irreversible island formation, $E_{L}^{a d s}$ more than $E_{L}^{A g}$ will impact the kinetics of film growth and thus the film morphology. All results are presented for Ag adatom(s) at their preferred adsorption sites. For either isolated adatoms or a complete layer adsorbed directly on the $\mathrm{NiAl}(110)$ substrate, this is the Ni-(short) bridge site $\mathrm{A}, \mathrm{B}, \mathrm{C}$, or D in Fig. 3(a). [10,11] For higher layers of the $\operatorname{Ag}(110)$ film, this is always the fourfold hollow site on the supporting $\operatorname{Ag}(110)$ layer.

Let $E_{L}^{\text {tot }}$ denote the total energy per surface unit cell of a supported $\mathrm{Ag}(110)$ film of $\mathrm{L}$ layers together with the $\mathrm{NiAl}(110)$ substrate. $\mathrm{L}=0$ corresponds to the clean substrate surface without any Ag layer. Then, it follows that $E_{L}=E_{L}^{\text {tot }}-E_{0}^{\text {tot }}$ is the total energy per unit cell associated with $\mathrm{Ag}$ atoms in the film and $E_{L}^{A g}=E_{L} / L$ for a $1 \times 1$ unit cell. The total energy per unit cell associated with atoms in layer L satisfies $\mu_{L} \equiv \Delta E_{L}=E_{L}-E_{L-1}$. A lower value of $\mu_{\mathrm{L}}$ reflects a more stable film of thickness L. More specifically, a film of thickness L will be stable against bifurcation to films of thickness $\mathrm{L} \pm 1$ if the stability index $\Delta \mu_{L}=\mu_{L+1}-\mu_{L}$ is positive. $E_{L}^{\text {tot }}$ and related quantities are calculated from a $1 \times 1$ supercell with a substrate of $11 \mathrm{NiAl}(110)$ layers using a $\mathbf{k}$ mesh is $15 \times 21 \times 1$. All atoms are relaxed except those in the bottom $6 \mathrm{NiAl}(110)$ layers. From Table II, $\mu_{\mathrm{L}}, \Delta \mu_{\mathrm{L}}$, and $E_{L}^{A g}$ all exhibit bilayer oscillations reflecting enhanced stability for layers of even thickness, behavior attributed to QSE. [10,11,20,21]

Next, we consider the adsorption energy of an isolated Ag adatom on an L-1 layer film. Let $E_{L-1}^{\text {tot+1 }}$ denote the total energy of the film of thickness L-1 together with the 
adsorbed isolated adatom and $\mathrm{E}_{\text {atom }}=-0.335 \mathrm{eV}$ (from DFT) denote the energy of a completely isolated atom. We then have that

$$
E_{L}^{a d s}=E_{L-1}^{t o t}+E_{\text {atom }}-E_{L-1}^{t o t+1}
$$

Results shown in Table II are from calculations using a $3 \times 4$ supercell, a $\mathbf{k}$ mesh is $4 \times 4 \times 1$, and relaxing all atoms except the bottom $\mathrm{NiAl}(110)$ layer. Results vary little for more $\mathbf{k}$ points or a thicker $\mathrm{NiAl}(110)$ substrate. $E_{L}^{a d s}$ also exhibits bilayer oscillations due to QSE: Stronger adsorption on films of odd thickness reflects enhanced stability of films with even thickness. Adsorption on top of a single-layer film is stronger than on the substrate by $\Delta \mathrm{E}_{\mathrm{ads}}=0.047 \mathrm{eV}$. Together with weaker adsorption on top of bilayer films, this promotes growth of bilayer islands and hinders population of the third layer.

The difference between energy per atom associated with a complete Lth layer and an isolated atom adsorbed in the same layer measures the total lateral attractive interaction energy per atom

$$
E_{L}^{\mathrm{int}}=-\left[\mu_{L}-\left(-E_{L}^{a d s}+E_{\text {atom }}\right)\right]
$$

as reported in Table II (and defined to be positive). $E_{L}^{\text {int }}$ is relevant for determining thin-film thermodynamics. To assess island nucleation behavior, instead one should consider the dominant nearest-neighbor $(\mathrm{NN})$ attraction, $\mathrm{E}_{\mathrm{b}}$ (defined to be positive) controlling the most stable isolated Ag dimers. Comparing the energies of an adsorbed dimer and of separated adsorbed constituent $\mathrm{Ag}$ atoms (with all $\mathrm{Ag}$ at preferred Ni-bridge sites), one obtains

$$
E_{b}=2 E_{0}^{t o t+1}-E_{0}^{t o t+2}-E_{0}^{t o t}
$$

where $E_{0}^{\text {tot+2 }}$ is the total energy of $\mathrm{NiAl}(110)$ substrate slab together with an adsorbed $\mathrm{Ag}$ dimer. 
Allowing complete relaxation of the adatoms in the dimer, one obtains a "strong" bond strength of $\mathrm{E}_{\mathrm{by}}=0.074 \mathrm{eV}$ for adatoms on nearest-neighbor Ni bridge sites in the [001] direction (y direction), e.g., A and B in Fig. 3(a). Similarly, one obtains a "weak" bond strength of $\mathrm{E}_{\mathrm{bx}}=0.005 \mathrm{eV}$ for nearest-neighbor Ni bridge sites in the [-110] direction ( $\mathrm{x}$ direction), e.g., A and D in Fig. 3(a). In these calculations, we used a $4 \times 5$ supercell, a k-point mesh of $6 \times 6 \times 1$, and relaxed substrate atoms except for the bottom layer of the 4-layer $\mathrm{NiAl}(110)$ substrate. Previous calculations comparing the energies for configurations of $\mathrm{Ag}$ addimers and separated $\mathrm{Ag}$ adatoms in a $3 \times 4$ supercell yielded $\mathrm{E}_{\mathrm{by}}=0.087 \mathrm{eV}$ and $\mathrm{E}_{\mathrm{bx}}=0.016$ eV. [11]

\subsection{Diffusion barriers}

The barriers for diffusion across terraces, $\mathrm{E}_{\mathrm{d}}$, along straight island edges, $\mathrm{E}_{\mathrm{e}}$, and around corners or kinks at island edges, $\mathrm{E}_{\mathrm{cr}}$, are key for controlling nucleation and lateral growth of islands. We obtain these barriers from a series of DFT + cNEB calculations. Fig. 3 shows a diffusion path for a $\mathrm{Ag}$ adatom on $\mathrm{NiAl}(110)$ surface from the preferred Ni-bridge

site, A, over a saddle point, $\mathrm{S}$, to a shallow local minimum, E, at a threefold Ni-Al-Al site. It can then move directly to the nearest Ni-bridge site B in the y direction, or pass through the Al-bridge site $\mathrm{F}$ and the local minimum $\mathrm{G}$ in the $\mathrm{x}$ direction to Ni-bridge sites $\mathrm{C}$ or $\mathrm{D}$. [11] Thus, the diffusion barrier is isotropic but the prefactor is $\sim 2$ times larger for the $\mathrm{x}$ direction versus y direction due to the rectangular unit cell. The DFT analysis shown in Fig. 3 yields $E_{d}=0.265 \mathrm{eV}$ using a supercell size of $2 \times 3$, a $\mathbf{k}$ mesh of $4 \times 4 \times 1$, and relaxing all atoms except the bottom layer of the 4-layer $\mathrm{NiAl}(110)$ slab. 
Diffusion along straight island edges is assessed from the energy landscape for a $\mathrm{Ag}$ atom moving along a fixed chain of Ag atoms. We assess diffusion around corners from consideration of a $\mathrm{Ag}$ atom moving around a single fixed $\mathrm{Ag}$ atom. Convergence tests of DFT + cNEB calculations show that a small $3 \times 4$ supercell is sufficient. Our calculations use a $\mathbf{k}$ mesh that is $4 \times 4 \times 1$ and substrate atoms are relaxed except the bottommost layer of the 4layer $\mathrm{NiAl}(110)$ substrate is fixed. We find that diffusion along horizontal edges is somewhat inhibited, so the barrier is a relevant model parameter. Diffusion along vertical edges which is facile (so model behavior is insensitive to the precise value of the barrier), and direct diffusion around corners is essentially inoperative (so the barrier is not relevant).

Fig. 4(a) shows two possible diffusion paths along a horizontal island edge. Fig. 4(b) shows DFT energies along path A-E-D path revealing a saddle point at site $S$ which determines the energy barrier of $0.289 \mathrm{eV}$. Fig. 4(b) also shows flatter energy along the A-F$\mathrm{D}$ path with an energy barrier of $0.367 \mathrm{eV}$ at $\mathrm{F}$ approximately equal to the sum $E_{d}+E_{b y}=0.265+0.087=0.352 \mathrm{eV}$. Thus, the diffusion path A-E-D dominates and the effective edge-diffusion barrier, $\mathrm{E}_{\mathrm{ex}}=0.289 \mathrm{eV}$, is comparable to $\mathrm{E}_{\mathrm{d}}$.

Fig. 5(a) shows the preferred diffusion path A-E-B along a vertical step edge. Edge diffusion is facile as the energy at the saddle point, $\mathrm{S}$, is only about $\mathrm{E}_{\mathrm{ey}} \sim 0.13 \mathrm{eV}$ above that for site A. This is not surprising since the diffusing Ag adatom at this transition state is strongly attracted to other Ag adatoms in the vertical chain representing the island edge. DFT indicates an energy for site E somewhat below that for A, an issue discussed further in Sec. 7. For corner rounding, Fig. 5(b) indicates one saddle point S1 with energy $0.20(0.27) \mathrm{eV}$ above that at A (B), and another S2 with energy $0.36(0.43)$ eV above A (B). Between S1 and $\mathrm{S} 2$ is a local minimum, E, with energy $0.12(0.19) \mathrm{eV}$ above A (B). Thus, a Ag adatom 
attempting to round the corner from A can readily hop to E but will tend not to continue directly to B. Instead, it would more easily hop to $\mathrm{C}$ then $\mathrm{F}$ then $\mathrm{B}$. So the direct pathway is not operative and corner rounding tends to occur via detachment and reattachment.

\section{Atomistic lattice-gas model and KMC simulation}

A realistic and comprehensive atomistic model for $\mathrm{Ag}$ island formation on $\mathrm{NiAl}(110)$ must include not only random deposition of $\mathrm{Ag}$, subsequent terrace diffusion and aggregation of $\mathrm{Ag}$ adatoms into islands, and diffusion along the edges of thus formed islands, but also detachment from island edges and interlayer transport. It is necessary to specify intralayer and interlayer hop rates for a large number of configurations with adatoms at or near island edges. Specification of all these barriers must be consistent with detailed balance and will thus reflect $\mathrm{Ag}$ adatom adsorption and interaction energies. Our general prescription for these barriers is crafted to incorporate precise DFT values for terrace and straight edge-diffusion processes. Below, $E_{b}^{\text {init }}=E_{b j}^{\text {init }}>0$ will denote the total lateral interaction energy before hopping for either a first-layer $(j=1)$ or second layer $(j=2)$ Ag adatom.

For intralayer hopping, "pure" edge hops where the Ag adatom has an NN edge atom both before and after hopping are described by a barrier $E_{e d g e}=E_{e y(x)}+\left(E_{b}^{i n i t}-E_{b x(y)}\right)$ for hopping in the $\mathrm{y}(\mathrm{x})$ direction. Thus, one has $E_{\text {edge }}=E_{e y(x)}$ for diffusion along straight edges. For all other intralayer hops, including terrace diffusion and attachment-detachment from island edges, one sets $E_{a c t}=E_{d}+E_{b}^{i n i t}$, so that the barrier for all attachment processes is $E_{a t t a c h}=E_{d}$. Detachment from y edges is facile with a barrier of $E_{a c t}=E_{d}+E_{b x}$ only slightly above $\mathrm{E}_{\mathrm{d}}$ due to weak interactions $\mathrm{E}_{\mathrm{bx}}$. The same low barrier applies for corner rounding from 
$\mathrm{y}$ edge to $\mathrm{x}$ edge. Detachment from kinks to terraces is operative, its barrier of $\mathrm{E}_{\mathrm{kink}}=\mathrm{E}_{\mathrm{d}}+\mathrm{E}_{\mathrm{by}}$ $+\mathrm{E}_{\mathrm{bx}}$ only slightly exceeding that for dissociation of the most stable dimers aligned in the $\mathrm{y}$ direction of $\mathrm{E}_{\mathrm{diss}}=\mathrm{E}_{\mathrm{d}}+\mathrm{E}_{\mathrm{by}}$.

For interlayer hopping, QSE becomes relevant. For upward hopping to the top of first-layer islands from their edges, i.e., for step climbing, we assign a barrier of

$$
E_{u p}=E_{d}+E_{b 1}^{\text {init }}+\delta
$$

where $\delta$ accounts for a possible additional step climbing barrier and here $E_{b 1}^{\text {init }}$ corresponds to first-layer adatom interactions (nonzero for upward hopping). $\delta$ will correspond to the standard definition of an Ehrlich-Schwoebel barrier in the absence of QSE. Thus, one has facile upward transport at straight y edges with barrier $E_{u p} \approx E_{d}+E_{b x}+\delta$ barely exceeding $\mathrm{E}_{\mathrm{d}}$ (assuming small $\delta$ ) due to weak interactions $\mathrm{E}_{\mathrm{bx}}$. For downward hopping, detailed-balance considerations impose a barrier of

$$
E_{\text {down }}=E_{d}+E_{b 2}^{\text {init }}+\delta+\Delta E_{a d s}
$$

where $\mathrm{E}_{\mathrm{d}}$ is again the diffusion barrier for $\mathrm{Ag}$ on $\mathrm{NiAl}(110)$ (not for $\mathrm{Ag}$ on top of single-layer islands) and $E_{b 2}^{\text {init }}$ corresponds to second-layer adatom interactions. The excess adsorption energy for $\mathrm{Ag}$ in the second layer relative to the first layer, $\Delta \mathrm{E}_{\mathrm{ads}}=0.047 \mathrm{eV}>0$, increases the barrier for downward hopping thus facilitating bilayer island growth. Fig. 6 illustrates the PES for an adatom traversing a step at the edge of a monolayer island (where $E_{b j}^{i n i t}=0$ for an isolated adatom).

KMC simulations were performed for our atomistic lattice-gas model of film growth on a substrate represented by a rectangular lattice of adsorption sites. The model includes all 
the above hopping processes with barriers as specified and a common prefactor of $10^{13} / \mathrm{s}$. Deposition at the experimental rate is operative until the target coverage of adsorbed $\mathrm{Ag}$ is reached after which only hopping processes are active. For simplicity, we treat second-layer sites as directly above first-layer sites rather than in their true fcc locations. This will not significantly impact shapes of larger islands or the degree of bilayer formation but may have some slight effect for small islands formed at low T. Diffusion processes in the second layer are treated by the same formulation used for the first layer. Our DFT analysis suggests that the strong bond $\mathrm{E}_{\mathrm{by}}$ is higher than in the first layer, so we have performed simulations with values equal to and above that for the first layer (and use $0.18 \mathrm{eV}$ unless otherwise stated). Other second-layer parameters are equated to firstlayer values. Table II shows adsorption in the third layer is $0.10 \mathrm{eV}$ weaker than in the second layer. Thus, formation of the third layer is strongly inhibited (and precluded in our modeling).

\section{KMC simulation results}

\subsection{Island formation during deposition}

Fig. 7 shows results for KMC simulation of film growth at 127, 140, and $150 \mathrm{~K}$, depositing $0.2 \mathrm{BL}$ of $\mathrm{Ag}$ using values of parameters indicated above except for increasing $\mathrm{E}_{\mathrm{bx}}$ to $0.036 \mathrm{eV}$, and setting $\delta=0$. (With a smaller $\mathrm{E}_{\mathrm{bx}}$, islands are too elongated.) Results reveal near-complete bilayer island formation even during deposition. Experimental STM images are also shown to demonstrate the success of the model in predicting trends in island density and shape with varying T. STM images are acquired roughly $10 \mathrm{~min}$ after completion of deposition. Thus, simulations are also continued into this postdeposition regime where there 
is a driving force for islands with only partial occupation of the second layer to evolve towards complete bilayer islands.

We have performed a substantial set of simulations varying key energetic parameters to assess their influence on the propensity for bilayer growth, see Table III. Three general trends are investigated: (i) varying lateral bonding while retaining $\Delta \mathrm{E}_{\mathrm{ads}}>0$ and setting the step climbing barrier $\delta=0$; (ii) setting $\Delta \mathrm{E}_{\mathrm{ads}}=0$; and (iii) increasing $\delta>0$. For (i), just slightly increasing the strong bond to $\mathrm{E}_{\mathrm{by}}=0.11 \mathrm{eV}$ results in partial bilayer islands at the end of deposition, but restructuring to almost complete bilayer islands still occurs in the next 10 min. Further increases in first-layer bonding inhibit even postdeposition formation of bilayer islands. For (ii), bilayer island formation does not occur even with stronger second-layer bonding, so $\Delta$ Eads $>0$ is key. For (iii), only partial bilayer islands occur at the end of deposition for $\delta \geq 0.02 \mathrm{eV}$, but complete bilayer islands still form within $10 \mathrm{~min}$ if $\delta \leq 0.04$ $\mathrm{eV}$.

We attribute facile bilayer island formation to the presence of anisotropic interactions and specifically to weak interactions in the [-110] direction. Diffusing atoms can easily ascend straight island edges aligned in the [001] direction. Also, atoms can escape first-layer kinks by breaking a single strong bond thereby enabling upward transport. To support these ideas, simulations for our model have been performed after first increasing $E_{b x 1}$ to equal $E_{b y 1}$ (cf. Table III) to produce isotropic first-layer interactions. Results shown in Fig. 8 reveal negligible bilayer island formation since kink adatoms are trapped in the first layer by two strong bonds. Of course, now shapes of the first-layer islands are isotropic. The nonzero second-layer population is presumably due primarily to direct deposition into this layer. 
One expects that island formation in this system at the lowest $\mathrm{T} \sim 130 \mathrm{~K}$ will be at least close to irreversible $(i=1)$. This might seem inconsistent with bilayer island formation. Irreversibility implies that the rate of dissociation of the most stable dimers is below the rate of aggregation (i.e., the rate at which diffusing atoms aggregate with dimers and other islands). Then, dimers convert to larger more stable islands before dissociation. For isotropic systems, typically growth does lead to more stable islands where essentially all atoms, including those at kink sites, are "locked in" by multiple strong bonds. However, in this anisotropic system, adatoms at kink sites have only a single strong bond and thus can escape on the time scale of deposition (which is much longer than the time scale of aggregation) to climb to the second layer as noted above.

Finally, we discuss further island growth shapes. Recall that bilayer Ag islands on $\mathrm{NiAl}(110)$ become increasingly elongated up to $300 \mathrm{~K}$, whereas monolayer Ag islands on $\mathrm{Ag}(110)$ become broader above about $220 \mathrm{~K}$. The bilayer structure of $\mathrm{Ag} / \mathrm{NiAl}(110)$ islands appears to "lock in" the growth structure and inhibits shape equilibration. To test this hypothesis, we have performed simulations using our model retaining the diffusion barriers and lateral interaction values for $\mathrm{Ag} / \mathrm{NiAl}(110)$, but inhibiting bilayer island formation. The results shown in Fig. 9 reveal that now island shapes do become broader even at quite low $\mathrm{T}$ analogous to $\mathrm{Ag} / \mathrm{Ag}(110)$ homoepitaxy. Simulation movies reveal that the monolayer islands at higher T are quite dynamic in contrast to the nearly "frozen" bilayer islands.

\subsection{Postdeposition coarsening}

Our simulation model has also been applied to study postdeposition coarsening of island distributions (retaining the same energetic parameters as for deposition studies). Our 
coarsening simulations incorporate exactly the same protocol for ramping up the temperature (following deposition at $130 \mathrm{~K}$ ) as used in experiment. See Fig. 10(a). Uncertainties in experimental temperatures (shown) are $\pm 2.5 \mathrm{~K}$. We find that deposition at $130 \mathrm{~K}$ and high $\mathrm{F}=0.056 \mathrm{ML} / \mathrm{s}$ produces islands with primarily monolayer structure. However, in about the first 10 min after deposition, these islands either dissolve or convert to primarily bilayer structure even with $\mathrm{T}$ held at $130 \mathrm{~K}$. When $\mathrm{T}$ is subsequently increased, there is significant coarsening. The decrease in simulated island density matches well the experimental observations.

We have already provided some discussion of uncertainties in experimental island densities in Sec. 4. One factor is the finite number, M, of islands counted (i.e., the finite sample size) for each data point. However, for our coarsening data, typically on the order of M 1000 islands are counted at $130 \mathrm{~K}$, and a few hundred for higher T, so corresponding uncertainties are small. A more significant factor is the difficulty in determining from STM images whether nearby islands are connected (and thus should be counted as a single island to be consistent with the percolation-like algorithm used in the simulations). The associated uncertainty is substantial in this coarsening study since the density of islands remains high and their size small (compared with deposition studies in Table I) at least up to 60 min or 170 $\mathrm{K}$. Based on detailed examination of the STM images, we assign uncertainties to the data in Fig. 10 of $\pm 15 \%$.

For the simulations, uncertainty comes only from sample size, and we estimate of the percentage uncertainty in the island density from $100(\mathrm{C} / \mathrm{M})^{1 / 2}$ using $\mathrm{C}=0.5$. Since these simulations for long times were very expensive, a small system size was used producing a larger uncertainty in the simulated island density of $\pm 12 \%$ at $130 \mathrm{~K}$ (where $\mathrm{M} 440$ ), 
increasing to $\pm 18 \%$ at $175 \mathrm{~K}$. Finally, we remark that the simulated $\mathrm{N}_{\mathrm{isl}} \sim 60 \times 10^{-3} \mathrm{~nm}^{-2}$ for deposition at $130 \mathrm{~K}$ with $\mathrm{F}=0.056 \mathrm{ML} / \mathrm{s}$ is consistent with the lower $\mathrm{N}_{\text {isl }} \sim 35 \times 10^{-3} \mathrm{~nm}^{-2}$ for a similar $\mathrm{T}=127 \mathrm{~K}$ in Table I where $\mathrm{F}$ is lower by an order of magnitude.

There exist extensive analyses of equilibrium island shapes and of coarsening for monolayer metal islands. [28,29] The equilibrium shape corresponds to minimizing the step (free) energy for fixed island size and the driving force for coarsening is a reduction in the overall step energy for a distribution of islands. Coarsening pathways include Ostwald ripening (OR: Net detachment of atoms from smaller islands and reattachment to larger ones) and Smoluchowski ripening (SR: Cluster diffusion and coalescence). Our simulations for bilayer $\mathrm{Ag}$ islands on $\mathrm{NiAl}(110)$ indicate a predominance of Ostwald ripening but experimental determination of the dominant pathway requires more detailed studies which we plan to perform.

For monolayer islands, one typically estimates the step energy as half the strength of the broken bonds per unit length of the step edge (noting that broken bonds are shared). Thus, for monolayer rectangular fcc(110) islands, their equilibrium aspect ratio equals the ratio of step energies for the two edges, which is accurately approximated by the ratio of the nearestneighbor interaction strengths for the two directions. For rectangular bilayer fcc(110) islands in the presence of QSE, the situation is more complicated. For simplicity, assume that these islands have a perfect (complete) bilayer structure. Then, it is important to note that as an island of fixed size changes shape, i.e., as it changes aspect ratio, the number of atoms in the second layer changes. The same is true if two smaller islands are combined to form a larger island with the same total number of atoms. Thus, the change in total energy of the system is impacted by the difference, $\Delta \mathrm{E}_{\mathrm{ads}}$, in adsorption energy for the substrate and on top of the 
first layer. If one defines an effective step energy for these bilayer islands which would describe the driving force for coarsening, then this would involve $\Delta \mathrm{E}_{\text {ads }}$ as well as the strengths of bonds broken at island edges in both the first and second layer. [50]

Next, we briefly discuss coarsening kinetics for OR. The effective barrier for coarsening via OR is usually written as $\mathrm{E}_{\mathrm{eff}}=\mathrm{E}_{\mathrm{d}}+\mathrm{E}_{\text {form }}+\delta_{\text {att }}$. Here, $\mathrm{E}_{\mathrm{d}}$ is the terrace diffusion barrier, $\mathrm{E}_{\text {form }}$ is the energy cost to detach an island atom (from a suitable kink site), and $\delta_{\text {att }}$ represents any effective attachment barrier. Although not tested, this result should apply for OR in anisotropic fcc(110) homoepitaxial systems, [51] with the caveat that unusual behavior can occur at low T. [52,53] For bilayer Ag islands on $\mathrm{NiAl}(110), \mathrm{E}_{\mathrm{d}}=0.265 \mathrm{eV}$ is the diffusion barrier for $\mathrm{Ag}$ on $\mathrm{NiAl}(110)$. Detachment from a second layer kink site will cost an energy $E_{\text {form }}=E_{b y 2}+E_{b x 2}+\Delta E_{\text {ads. }}$. If there exists a substantial step climbing barrier, then one has a nonzero $\delta_{\text {att }} \approx \delta$. A more detailed discussion will be presented elsewhere.

Finally, we consider the possibility of an unusual dependence of coarsening kinetics on flux. This is motivated by observations for quantum islands of $\mathrm{Pb}$ on $\mathrm{Si}(111)$ : Deposition with higher F naturally produce a higher initial island density but surprisingly a lower postcoarsening island density. [54] The reason is that in this system, high-F deposition produces a larger population of islands with unstable heights which are susceptible to rapid coarsening. [30] For the $\mathrm{Ag} / \mathrm{NiAl}(110)$ system, deposition with higher $\mathrm{F}$ can produce a higher population of monolayer or incomplete bilayer islands which might also be amenable to rapid coarsening. Simulation results shown in Fig. 11 do reveal more rapid coarsening for higher $\mathrm{F}$ but postcoarsening island densities are still higher. 


\section{Discussion and summary}

Detailed atomistic modeling has been presented that successfully describes and elucidates the kinetics of facile Ag bilayer island formation on $\mathrm{NiAl}(110)$ at low $\mathrm{T}$. In addition, the same model is effective in describing postdeposition coarsening. This success reflects the feature that the model accurately captures both thermodynamic and kinetic aspects of this system, the choice of energetic parameters being guided by extensive DFT analysis.

Our modeling does likely oversimplify the description of some finer details of the structure and kinetics in this $\mathrm{Ag} / \mathrm{NiAl}(110)$ system. Recall that our DFT analysis in Sec. 4 indicated a slightly lower energy of a diffusing atom near an Al-bridge site close to a vertical island edge [E in Fig. 5(a)] rather than at the standard Ni-bridge site. Similarly, we find that for an adjacent vertical pair of Ag atoms at this step edge, Al-bridge site configuration close to the island edge is slightly preferred over the standard Ni-bridge sites. These motifs reflect the "hex" structure described in Appendix A.

Thus, it is plausible that in the initial stages of $\mathrm{Ag}$ adatom aggregation to form islands, monolayer structures with hex-type motifs do appear. However, except perhaps for very low $\mathrm{T}$, one expects that such island structures if formed must readily convert to bilayer fcc(110) structures as they grow larger, their adatoms returning to standard adsorption sites. In this case, the current model would not precisely describe the initial stages of island formation, but would effectively describe subsequent lateral growth and bilayer development. A more precise description would require off-lattice or multisite lattice-gas modeling. Given this more complex picture, one might anticipate that that deposition at very low $\mathrm{T}$ and higher flux could trap islands in the local hex structure at least initially. Our STM data for 
deposition at $130 \mathrm{~K}$ with high $\mathrm{F}=0.056 \mathrm{ML} / \mathrm{s}$ up to $\sim 15 \mathrm{~min}$ after deposition provides some indication that this is the case (although image quality is insufficient for definitive determination of structure).

While many other systems exhibit bilayer or multilayer quantum islands, a detail atomistic-level characterization of the kinetics has been lacking. For $\mathrm{Ag} / \mathrm{NiAl}(110)$, the strong anisotropy in bonding is a key factor responsible for the observed facile bilayer island formation. The feature that strong anisotropy facilitates growth and relaxation kinetics for bilayer and multilayer islands should apply to other heteroepitaxial systems.

\section{Acknowledgements}

The modeling component of this work by Y.H. and J.W.E. was supported by NSF under Grant No. CHE-0809472, as were experimental contributions of F.Q. and P.A.T. Computational support at NERSC was provided by the U.S. DOE. Experimental contributions of B.U., D.J., and C.J.J. were supported by U.S. DOE BES-Materials Sciences. DFT analysis by D.J.L. was supported by U.S. DOE BES-Chemical Sciences. The work was performed at Ames Laboratory which is operated for the U.S. DOE by ISU under Contract No. DE-AC02-07CH11358.

\section{Appendix A: Structure of Ag overlayers on $\mathrm{NiAl}(110)$}

Fig. 12(a)-12(c) show monolayer versions of various low-energy Ag film structures on $\mathrm{NiAl}(110)$ as determined by DFT analysis. Fig. 12(a) shows a monolayer $\mathrm{Ag}(110)$ structure. We claim that observed Ag islands correspond to the bilayer version of this structure (which is more stable than the monolayer version) with second-layer atoms at the 
fourfold hollow sites. See also Ref. 10. The height of monolayer (bilayer) fcc(110) structures is $0.21 \mathrm{~nm}(0.34 \mathrm{~nm})$, the latter matching experimental bilayer island height. Fig. 12(b) shows a monolayer "double-chain" structure where rows of atoms in the (110) structure have paired. The height of this monolayer structure is $0.22 \mathrm{~nm}$, too low to match experiment. The bilayer version of this structure with second-layer double chains between first-layer chains is not very stable. Fig. 12(c) shows a monolayer "square-hex" structure with height of $0.23 \mathrm{~nm}$, too low to match experiment. The bilayer version of this structure has the second-layer hex motifs sitting above monolayer square motifs and visa versa. It is quite stable but has a height of $0.49 \mathrm{~nm}$, too high to match experiment.

\section{Appendix B: DFT analysis of Ni substitutional defect on NiAl(110)}

A Ni-substitutional defect on $\mathrm{NiAl}(110)$, where an $\mathrm{Al}$ atom in the surface layer is replaced by Ni, is shown in Fig. 13. Below, we calculate modified adsorption energies for a $\mathrm{Ag}$ adatom and addimer in the vicinity of this defect. In these DFT calculations, we use a $4 \times 5$ supercell, a k-point mesh of $6 \times 6 \times 1$, and relax all atoms except the bottommost layer of the 4 layer $\mathrm{NiAl}(110)$ substrate.

For a Ag adatom, the preferred adsorption site is at D shown in Fig. 13 (shifted $\sim 0.017 \mathrm{~nm}$ toward the $\mathrm{Ni}$ defect from the Ni-bridge site). The corresponding adsorption energy $E_{d e f}^{a d s}=2.484 \mathrm{eV}$, which is larger by $\Delta \mathrm{E}_{\mathrm{def}}=0.103 \mathrm{eV}$ than $\mathrm{E}_{\mathrm{ads}}=2.381 \mathrm{eV}$ (see Table II, and also note that subscript " $\mathrm{L}=1$ " has been omitted here) for the preferred adsorption site, e.g., site A in Fig. 3(a), on a defect-free surface. Similar to the adsorption energy for an adatom, we define the total adsorption energy for an addimer, $\mathrm{E}^{\text {adsd }}$, by correspondingly 
replacing "adatom" with "addimer" in Eq. (2). For a defect-free $\mathrm{NiAl}(110)$ surface, the most stable Ag addimer sits on sites A and B in Fig. 3(a) with $\mathrm{E}^{\mathrm{adsd}}=4.839 \mathrm{eV}$. For a Ni defect, the preferred adsorption sites are $\mathrm{E}$ and $\mathrm{F}$ shown in Fig. 13. The corresponding $E_{\text {def }}^{\text {ads }}=5.050 \mathrm{eV}$, which is $0.211 \mathrm{eV}$ larger. The preferred adsorption sites $\mathrm{E}$ and $\mathrm{F}$ correspond to an addimer bond length reduced by $\sim 25 \%$ relative to its standard value. Thus, the effective bond strength for the dimer at the defect is $\mathrm{E}_{\mathrm{bd}}=\mathrm{E}_{\mathrm{b}}+0.211 \mathrm{eV}-\Delta \mathrm{E}_{\mathrm{def}}=0.195 \mathrm{eV}$.

\section{Appendix C: Rate equation analysis of defect-enhanced nucleation}

The decrease in experimental island density, $\mathrm{N}_{\mathrm{isl}}$, with increasing higher $\mathrm{T}$ is slower than predicted by our model for homogeneous nucleation. This may due to the neglect of heterogeneous nucleation at defects such as the Ni-substitutional point defect. An STM image of point defects obtained after one standard $\mathrm{NiAl}(110)$ sample preparation procedure is shown as an inset in Fig. 14. As noted in Sec. 3, this observation of defects is consistent with those of other groups. Our proposal for competitive homogeneous and heterogeneous nucleation is assessed by developing a mean-field rate equation (RE) model. The goal is to identify viable ranges for the defect density and adatom bonding which lead to enhanced nucleation.

The key ingredients of the model are as follows: (i) reversible homogeneous nucleation of $\mathrm{Ag}$ islands on terraces controlled by terrace diffusion with barrier $\mathrm{E}_{\mathrm{d}}=0.265 \mathrm{eV}$ and a strong adatom bond of $E_{b}=0.087 \mathrm{eV}$. For simplicity, we assign a critical size of $i=3$ (i.e., islands of size more than 3 atoms are stable). We assign capture numbers for substable islands as $\sigma_{\mathrm{s}<4}=1$ and for all stable islands as $\sigma_{\mathrm{x}}=5$ (a choice based on simulation analyses). 
Rates for detachment from islands are governed by detailed balance and thus involve $\mathrm{E}_{\mathrm{b}}$. Since mean-field modeling artificially enhances dimer dissociation rates, [38] we further reduce detachment rates by a factor of $\mathrm{c}=0.5$ to mimic exact behavior. (ii) Reversible heterogeneous nucleation of $\mathrm{Ag}$ islands at defects is partly due to enhanced binding of adatoms at defects by an amount $\Delta \mathrm{E}_{\text {def. }}$. Adatoms can thus reversibly attach and detach from defect sites, where the detachment rate is influenced by $\Delta \mathrm{E}_{\text {def }}$ according to detailed balance. In addition, we allow for stronger adatom bonding at defects with strength $E_{b d}>E_{b}$. Capture numbers, the critical size, and the factor $c$ are chosen as for homogeneous nucleation, and we choose the capture number for empty defects as $\sigma_{0}=1$.

It is convenient to introduce the following notation. First, let $\mathrm{N}_{\mathrm{s}}$ and $\mathrm{M}_{\mathrm{s}}$ denote the density of islands of size $\mathrm{s}$ on terraces and at defects, respectively, and let $\mathrm{N}_{\mathrm{x}}=\mathrm{N}_{4}+\mathrm{N}_{5}+\ldots$ and $\mathrm{M}_{\mathrm{x}}=\mathrm{M}_{4}+\mathrm{M}_{5}+\ldots$ denote the corresponding densities of stable islands. Thus, if $\mathrm{M}$ denotes the density of defects, then $M_{0}=M-M_{1}-M_{2}-M_{3}-M_{x}>0$ denotes the density of defects free of $A g$ atoms of islands. Second, let $h \equiv v e^{-\beta E_{d}}$ denote the terrace hop rate. Then, we introduce rates $\mathrm{K}_{\mathrm{s}}=\sigma_{\mathrm{s}} \mathrm{hN}_{1} \mathrm{~N}_{\mathrm{s}}$ and $\mathrm{K}_{\mathrm{s}}^{\mathrm{d}}=\sigma_{\mathrm{s}} \mathrm{hN} \mathrm{N}_{1} \mathrm{M}_{\mathrm{s}}$ for aggregation of diffusing adatoms with terrace and defect islands of size s4, respectively, and $F_{s}=c \sigma_{s} h e^{-\beta E_{b}} N_{s}$ and $F_{s}^{e}=c \sigma_{s} h e^{-\beta E_{b d}} M_{s}$ for detachment of adatoms from terrace and defect islands of size s, respectively. For rates of aggregation with stable islands, the subscript $\mathrm{s}$ is replaced by $\mathrm{x}$. Then, one obtains the equations

$$
\begin{aligned}
& \frac{d N_{1}}{d t}=F\left(1-\frac{\theta}{2}\right)-2 K_{1}-K_{2}-K_{3}-K_{x}-K_{0}^{d}-K_{1}^{d}-K_{2}^{d}-K_{3}^{d} \\
& -K_{x}^{d}+2 F_{2}+F_{3}+F_{2}^{d}+F_{3}^{d}+\sigma_{0} h e^{-\beta \Delta E_{d e f}} M_{1}
\end{aligned}
$$




$$
\begin{gathered}
\frac{d M_{1}}{d t}=K_{0}^{d}-K_{1}^{d}+F_{2}^{d}-\sigma h e^{-\beta \Delta E_{d e f}} M_{1} \\
\frac{d N_{2}}{d t}=K_{1}-K_{2}-F_{2}+F_{3} \\
\frac{d M_{2}}{d t}=K_{1}^{d}-K_{2}^{d}-F_{2}^{d}+F_{3}^{d} \\
\frac{d N_{3}}{d t}=K_{2}-K_{3}-F_{3}, \frac{d M_{3}}{d t}=K_{2}^{d}-K_{2}^{d}-F_{3}^{d} \\
\frac{d N_{x}}{d t}=K_{3}, \frac{d M_{x}}{d t}=K_{3}^{d} \quad(\mathrm{C} 6)
\end{gathered}
$$

To recover experimental behavior for $\mathrm{T}$ up to $190 \mathrm{~K}$, we can choose $\Delta \mathrm{E}_{\mathrm{def}}=0.103 \mathrm{eV}$ and $\mathrm{E}_{\mathrm{bd}}=0.195 \mathrm{eV}$, from DFT values, and a defect density of $\mathrm{M}=0.001 /$ site $=0.0085 \mathrm{~nm}^{-2}$ (chosen below the typical island density for lower $\mathrm{T}$ ). With this parameter choice, $\mathrm{N}_{\text {isl }}$ still decreases more quickly than in experiment above $190 \mathrm{~K}$ (shown as RE1 in Fig. 14). If one reduces the value of $\mathrm{E}_{\mathrm{bd}}$ for trimers at defects to equal $\mathrm{E}_{\mathrm{b}}$, then the decrease in $\mathrm{N}_{\mathrm{isl}}$ is more dramatic (RE2 in Fig. 14). On the other hand, inhibition of the rapid decrease in $\mathrm{N}_{\text {isl }}$ just above $190 \mathrm{~K}$ can be achieved by increasing $\mathrm{E}_{\mathrm{bd}}$. However, the discrepancy between the model and experiment is likely real, perhaps reflecting the neglect of heterogeneous nucleation at other types of defects.

We offer one final comment on low-T behavior, specifically regarding comparison of predictions of the full model, behavior for $\mathrm{i}=1$, and RE predictions. Reversibility around 140 $\mathrm{K}$ would tend to give steeper Arrhenius slope than E $=\mathrm{E}_{\mathrm{d}} / 3$ for an $\mathrm{i}=1$ model. However, there is also some postdeposition coarsening of the small islands formed in experiment at low $\mathrm{T}$ which lowers the measured density thus the 
Arrhenius slope at low T. Due to these competing effects, the slope appears to match that for $\mathrm{i}=1$ behavior.

\section{References}

[1] E. Bauer, Z. Kristallogr. 110, 372 (1958).

[2] P. J. Schmitz, W.-Y. Leung, G. W. Graham, and P. A. Thiel, Phys. Rev. B 40, 11477 (1989).

[3] S.-L. Chang, J.-M. Wen, P. A. Thiel, S. Günther, J. A. Meyer, and R. J. Behm, Phys. Rev. B 53, 13747 (1996).

[4] R. Pentcheva and M. Scheffler, Phys. Rev. B 61, 2211 (2000).

[5] B. Voigtländer, G. Meyer, and N. M. Amer, Phys. Rev. B 44, 10354 (1991).

[6] C. Tölkes, P. Zeppenfeld, M. A. Krzyzowski, R. David, and G. Comsa, Phys. Rev. B 55,13932 (1997).

[7] H. W. Chang, F. T. Yuan, Y. D. Yao, W. Y. Cheng, W. B. Su, C. S. Chang, C. W. Lee, and W. C. Cheng, J. Appl. Phys. 100, 084304 (2006).

[8] H. Hoster, T. Iwasita, H. Baumgärtner, and W. Vielstich, Phys. Chem. Chem. Phys. 3, 337 (2001).

[9] A. Verdini, L. Floreano, F. Bruno, D. Cvetko, A. Morgante, F. Bisio, S. Terreni, and M. Canepa, Phys. Rev. B 65, 233403 (2002).

[10] B. Unal, F. Qin, Y. Han, D.-J. Liu, D. Jing, A. R. Layson, C. J. Jenks, J. W. Evans, and P. A. Thiel, Phys. Rev. B 76, 195410 (2007).

[11] Y. Han, B. Unal, F. Qin, D. Jing, C. J. Jenks, D.-J. Liu, P. A. Thiel, and J. W. Evans, Phys. Rev. Lett. 100, 116105 (2008). 
[12] A. R. Smith, K.-J. Chao, Q. Niu, and C.-K. Shih, Science 273, 226 (1996).

[13] D.-A. Luh, T. Miller, J. J. Paggel, M. Y. Chou, and T.-C. Chiang, Science 292, 1131 (2001).

[14] K. L. Man, Z. Q. Qiu, and M. S. Altman, Phys. Rev. Lett. 93, 236104 (2004).

[15] B. J. Hinch, C. Koziol, J. P. Toennies, and G. Zhang, Europhys. Lett. 10, 341 (1989).

[16] R. Otero, A. L. Vázquez de Parga, and R. Miranda, Phys. Rev. B 66, 115401 (2002).

[17] V. Fournée, H. R. Sharma, M. Shimoda, A. P. Tsai, B. Unal, A. R. Ross, T. A. Lograsso, and P. A. Thiel, Phys. Rev. Lett. 95, 155504 (2005).

[18] B. Ünal, V. Fournée, P. A. Thiel, and J. W. Evans, Phys. Rev. Lett. 102, 196103 (2009).

[19] F. K. Schulte, Surf. Sci. 55, 427 (1976).

[20] Y. Han, J. W. Evans, and D.-J. Liu, Surf. Sci. 602, 2532 (2008).

[21] Y. Han and D.-J. Liu, Phys. Rev. B 80, 155404 (2009).

[22] R. A. Miron and K. A. Fichthorn, Phys. Rev. B 72, 035415 (2005).

[23] Z. Kuntová, M. C. Tringides, and Z. Chvoj, Phys. Rev. B 78, 155431 (2008).

[24] Y. Han, F. Liu, S.-C. Li, J.-F. Jia, Q.-K. Xue, and B.-J. Lee, Appl. Phys. Lett. 92, 021909 (2008).

[25] Y. Han, G.-H. Lu, B.-J. Lee, and F. Liu, Surf. Sci. 602, 2284 (2008).

[26] S.-C. Li, Y. Han, J.-F. Jia, Q.-K. Xue, and F. Liu, Phys. Rev. B 74, 195428 (2006).

[27] M. Zinke-Allmang, L. C. Feldman, and M. H. Grabow, Surf. Sci. Rep. 16, 377 (1992).

[28] K. Morgenstern, Phys. Status Solidi B 242, 773 (2005).

[29] P. A. Thiel, M. Shen, D.-J. Liu, and J. W. Evans, J. Phys. Chem. C 113, 5047 (2009). 
[30] M. Li, J. W. Evans, C. Z. Wang, M. Hupalo, M. C. Tringides, T.-L. Chan, and K. M. Ho, Surf. Sci. 601, L140 (2007).

[31] G. Kresse and J. Hafner, Phys. Rev. B 47, 558 (1993).

[32] J. P. Perdew, K. Burke, and M. Ernzerhof, Phys. Rev. Lett. 77, 3865 (1996).

[33] G. Kresse and D. Joubert, Phys. Rev. B 59, 1758 (1999).

[34] G. Henkelman and H. Jónsson, J. Chem. Phys. 113, 9978 (2000).

[35] M. Prutton, Introduction to Surface Physics (Oxford University Press, Oxford, 1987).

[36] D. K. Flynn, W. Wang, S. L. Chang, M. C. Tringides, and P. A. Thiel, Langmuir 4, 1096 (1988).

[37] H. C. Kang, D. K. Flynn-Sanders, P. A. Thiel, and J. W. Evans, Surf. Sci. 256, 205 (1991).

[38] J. W. Evans, P. A. Thiel, and M. C. Bartelt, Surf. Sci. Rep. 61, 1 (2006).

[39] C.-M. Zhang, M. C. Bartelt, J.-M. Wen, C. J. Jenks, J. W. Evans, and P. A. Thiel, Surf. Sci. 406, 178 (1998).

[40] J. A. Venables, Philos. Mag. 27, 697 (1973).

[41] M. C. Bartelt and J. W. Evans, Surf. Sci. 298, 421 (1993).

[42] M. C. Bartelt, L. S. Perkins, and J. W. Evans, Surf. Sci. 344, L1193 (1995).

[43] Z. Song, J. I. Pascual, H. Conrad, K. Horn, and H.-P. Rust, Appl. Phys. A: Mater. Sci. Process. 72, S159 (2001).

[44] W. Ho (private communication).

[45] G. A. Eckstein, S. Maupai, A. S. Dakkouri, M. Stratmann, M. Nielsen, M. M. Nielsen, R. Feidenhans'1, J. H. Zeysing, O. Bunk, and R. L. Johnson, Phys. Rev. B 60, 8321 (1999). 
[46] J. P. Pierce, N. C. Bartelt, and K. F. McCarty, Phys. Rev. Lett. 99, 026101 (2007). [47] T. M. Wallis, N. Nilius, and W. Ho, Phys. Rev. Lett. 89, 236802 (2002).

[48] N. Nilius, T. M. Wallis, and W. Ho, Appl. Phys. A: Mater. Sci. Process. 80, 951 (2005).

[49] C. Chen, C. A. Bobisch, and W. Ho, Science 325, 981 (2009).

[50] For layer-independent lateral interactions, one finds an aspect ratio for equilibrated bilayer islands of Ly $/ \mathrm{Lx} \approx($ Eby $+\Delta$ Eads $/ 4) /($ Ebx $+\Delta$ Eads $/ 4)$ with $\mathrm{Lx}$ and Ly measured in atoms. A detailed analysis will be reported elsewhere.

[51] P. A. Thiel, M. Shen, D.-J. Liu, and J. W. Evans, J. Vac. Sci. Technol. A (to be published).

[52] K. Morgenstern, E. Laegsgaard, I. Stensgaard, and F. Besenbacher, Phys. Rev. Lett. 83, 1613 (1999).

[53] Y. Yao, P. Ebert, M. Li, Z. Zhang, and E. G. Wang, Phys. Rev. B 66, 041407(R) (2002).

[54] C. A. Jeffrey, E. H. Conrad, R. Feng, M. Hupalo, C. Kim, P. J. Ryan, P. F. Miceli, and M. C. Tringides, Phys. Rev. Lett. 96, 106105 (2006). 


\section{Tables}

Table I. Experimental Ag island density $\mathrm{N}_{\text {isl }}$ (in units of $10^{-3} \mathrm{~nm}^{-2}$ ) versus deposition temperature $\mathrm{T}$ (in units of $\mathrm{K}$ ). The number, $\mathrm{M}$, of islands counted at each $\mathrm{T}$ is also shown. Adsorption site (unit cell) area is $0.118 \mathrm{~nm}^{2}$. Note that we use more data than that in our previous analysis (Ref. 11) resulting in slightly different values for $\mathrm{N}_{\text {isl }}$.

\begin{tabular}{|c|c|c|c|c|c|c|c|c|c|}
\hline $\mathrm{T}$ & 127 & 140 & 150 & 175 & 190 & 225 & 250 & 275 & 300 \\
\hline $\mathrm{N}_{\text {isl }}$ & 35 & 18.0 & 12.6 & 4.67 & 3.49 & 1.27 & 0.66 & 0.21 & 0.079 \\
\hline $\mathrm{M}$ & 1620 & 770 & 910 & 480 & 1530 & 710 & 490 & 320 & 400 \\
\hline
\end{tabular}


Table II. Magnitudes of various energies in electron volt (per surface unit cell) versus film thickness L in layers. Energies were determined from DFT calculations for $\mathrm{Ag}(110) / \mathrm{NiAl}(110)$ or $\mathrm{Ag} / \mathrm{Ag}(110) / \mathrm{NiAl}(110)$ systems. A star denotes a value obtained by extrapolation to $\mathrm{L} \rightarrow \infty$.

\begin{tabular}{|c|c|c|c|c|c|}
\hline $\mathrm{L}$ & $E_{L}^{A g}$ & $\mu_{L}=E_{L}-E_{L-1}$ & $\Delta \mu_{L}$ & $E_{L}^{a d s}$ & $E_{L}^{\text {int }}$ \\
\hline 1 & -2.838 & -2.838 & -0.0491 & 2.381 & 0.122 \\
\hline 2 & -2.863 & -2.888 & 0.1339 & 2.428 & 0.124 \\
\hline 3 & -2.862 & -2.754 & -0.1312 & 2.324 & 0.095 \\
\hline 4 & -2.841 & -2.885 & 0.1060 & 2.428 & 0.122 \\
\hline 5 & -2.829 & -2.779 & -0.0968 & 2.294 & 0.150 \\
\hline 6 & -2.836 & -2.876 & 0.0821 & 2.412 & 0.129 \\
\hline 7 & -2.830 & -2.793 & -0.0658 & 2.265 & 0.194 \\
\hline$\infty$ & -2.828 & -2.832 & 0 & 2.324 & 0.173 \\
\hline
\end{tabular}


Table III. KMC simulation results assessing the extent of second-layer formation as a function of key energetic parameters (in millielectron volt) at $140 \mathrm{~K}$ with $\mathrm{F}=0.0066$ $\mathrm{ML} / \mathrm{s} . \mathrm{N}_{2}$ is the number of second-layer atoms and $\mathrm{N}_{1}$ is the number of exposed firstlayer atoms, so that the total number of adatoms is $\mathrm{N}_{1}+2 \mathrm{~N}_{2}=1475$ when deposition is completed. We show the ratio $\mathrm{R}=\mathrm{N}_{2} / \mathrm{N}_{1}$ both at the end of deposition (at $\sim 45 \mathrm{~s}$ ) and later (at $\sim 595 \mathrm{~s}$ ). Subscripts 1 and 2 of $\mathrm{E}_{\mathrm{bx}}$ or $\mathrm{E}_{\mathrm{by}}$ denote $\mathrm{Ag}$ interactions for adsorption on top of the $\mathrm{NiAl}(110)$ substrate surface and in the first $\mathrm{Ag}(110)$ layer, respectively.

\begin{tabular}{|c|c|c|c|c|c|c|c|}
\hline $\mathrm{E}_{\mathrm{by} 1}$ & $\mathrm{E}_{\mathrm{bx} 1}$ & $\mathrm{E}_{\mathrm{by} 2}$ & $\mathrm{E}_{\mathrm{bx} 2}$ & $\Delta \mathrm{E}_{\mathrm{ads}}$ & $\delta$ & $\mathrm{R}(\sim 45 \mathrm{~s})$ & $\mathrm{R}(\sim 595 \mathrm{~s})$ \\
\hline 86 & 36 & 140 & 36 & 47 & 0 & $687 / 101$ & $737 / 1$ \\
\hline 86 & 36 & 180 & 36 & 47 & 0 & $689 / 97$ & $737 / 1$ \\
\hline 110 & 36 & 180 & 36 & 47 & 0 & $609 / 257$ & $735 / 5$ \\
\hline 126 & 36 & 180 & 36 & 47 & 0 & $358 / 759$ & $607 / 261$ \\
\hline 110 & 50 & 180 & 50 & 47 & 0 & $464 / 547$ & $706 / 63$ \\
\hline 120 & 50 & 180 & 50 & 47 & 0 & $343 / 789$ & $527 / 421$ \\
\hline 86 & 86 & 180 & 36 & 47 & 0 & $266 / 943$ & $401 / 673$ \\
\hline 86 & 86 & 86 & 86 & 47 & 0 & $253 / 969$ & $463 / 603$ \\
\hline 86 & 36 & 187 & 36 & 0 & 0 & $18 / 1439$ & $668 / 139$ \\
\hline 86 & 36 & 180 & 36 & 47 & 20 & $564 / 347$ & $736 / 3$ \\
\hline 86 & 36 & 180 & 36 & 47 & 40 & $307 / 861$ & $714 / 47$ \\
\hline 86 & 36 & 180 & 36 & 47 & 50 & $212 / 1051$ & $619 / 237$ \\
\hline
\end{tabular}




\section{Figure captions}

Fig. 1. STM images of Ag bilayer islands formed on NiAl(110) surface by Ag deposition with flux $\mathrm{F}=3.3 \times 10^{-3} \mathrm{BL} / \mathrm{s}$ at (a) $127 \mathrm{~K}$, (b) $140 \mathrm{~K}$, (c) $190 \mathrm{~K}$, (d) $250 \mathrm{~K}$, and (e) 300 K. Image sizes are $72.5 \times 72.5 \mathrm{~nm}^{2}$ for (a)-(d) and $500 \times 500 \mathrm{~nm}^{2}$ for (e). Note that the [001] crystallographic direction of the $\mathrm{NiAl}(110)$ substrate is in the direction of elongation of the Ag islands.

Fig. 2. STM images of Ag bilayer island coarsening following formation by Ag deposition on $\mathrm{NiAl}(110)$ surface at $130 \mathrm{~K}$ with flux $\mathrm{F}=2.8 \times 10^{-2} \mathrm{BL} / \mathrm{s}$. The time after deposition at the corresponding temperature is (a) $0 \mathrm{~min}$ at $130 \mathrm{~K}$, (b) $67 \mathrm{~min}$ at $175 \mathrm{~K}$, (c) $100 \mathrm{~min}$ at $197 \mathrm{~K}$, (d) $162 \mathrm{~min}$ at $234 \mathrm{~K}$, (e) $203 \mathrm{~min}$ at $252 \mathrm{~K}$, and (f) $277 \mathrm{~min}$ at $274 \mathrm{~K}$, respectively. The size of any image is $100 \times 100 \mathrm{~nm}^{2}$. Note that the [001] crystallographic direction of the $\mathrm{NiAl}(110)$ substrate is in the direction of elongation of the Ag islands.

Fig. 3. (a) Terrace diffusion paths from DFT $+\mathrm{cNEB}$ calculations for a Ag adatom on a NiAl(110) surface. (b) The corresponding cNEB energy curves.

Fig. 4. (a) Diffusion paths along a horizontal Ag island edge represented by a fixed horizontal Ag chain. (b) The DFT+cNEB energy curves correspond to two possible diffusion paths in (a).

Fig. 5. (a) Diffusion path along a vertical Ag island edge represented by a fixed vertical Ag chain. (b) Diffusion path around an island corner represented by a single fixed $\mathrm{Ag}$ adatom.

Fig. 6. Schematic of the PES for an adatom traversing the step edge of a single-layer island. 
Fig. 7. Images $\left(46 \times 46 \mathrm{~nm}^{2}\right)$ of $\mathrm{Ag}$ islands on $\mathrm{NiAl}(110)$ for $\mathrm{F}=0.0066 \mathrm{ML} / \mathrm{s}$. From left to right: $\mathrm{KMC}$ simulation with $\mathrm{E}_{\mathrm{by}}=0.086 \mathrm{eV}, \mathrm{E}_{\mathrm{bx}}=0.036 \mathrm{eV}$, and $\delta=0$ after deposition of $0.1 \mathrm{BL}, 0.2 \mathrm{BL}$, and $10 \mathrm{~min}$ later; STM image 10 min after deposition of $0.2 \mathrm{BL}$ $(\mathrm{I}=0.5 \mathrm{nA}, \mathrm{V}=+1.0 \mathrm{~V})$. Light gray (white) denotes first (second) layer of Ag. [(a)-(d)] $\mathrm{T}=127 \mathrm{~K} ;[(\mathrm{e})-(\mathrm{h})] \mathrm{T}=140 \mathrm{~K}$; and $[(\mathrm{i})-(\mathrm{l})] \mathrm{T}=150 \mathrm{~K}$.

Fig. 8. KMC simulated images $\left(27 \times 19 \mathrm{~nm}^{2}\right)$ of island formation with isotropic interactions at $\mathrm{T}=140 \mathrm{~K}$. All parameters are the same as those of Fig. 7 except $\mathrm{E}_{\mathrm{bx}}$ is set equal to $\mathrm{E}_{\mathrm{by}}=0.086 \mathrm{eV}$. Light gray (white) denotes first (second) layer of Ag. (a) 0.1 BL and (b) $0.2 \mathrm{BL}$.

Fig. 9. KMC images $\left(46 \times 46 \mathrm{~nm}^{2}\right)$ of single-layer island formation with anisotropic interactions. All parameters are the same as those of Fig. 7 except $\delta$ is set to infinity to inhibit hopping up of first-layer adatoms. Also, adatoms directly deposited on top of the first $\mathrm{Ag}(110)$ layer are neglected. (a) $\mathrm{T}=127 \mathrm{~K}$ and $\theta=0.2 \mathrm{ML}$; (b) $\mathrm{T}=140 \mathrm{~K}$ and $\theta=0.2 \mathrm{ML} ;(\mathrm{c}) \mathrm{T}=150 \mathrm{~K}$ and $\theta=0.2 \mathrm{ML}$; (d) $\mathrm{T}=175 \mathrm{~K}$ and $\theta=0.2 \mathrm{ML}$; and (e) $\mathrm{T}=190 \mathrm{~K}$ and $\theta=0.13 \mathrm{ML}$.

Fig. 10. (a) Experimental temperature points as a function of time $t$ after the deposition ( $\mathrm{I}=0.5$ $\mathrm{nA}, \mathrm{V}=+1.0 \mathrm{~V}$, and $\mathrm{F}=0.056 \mathrm{ML} / \mathrm{s})$ of $0.14 \mathrm{BL} \mathrm{Ag}$ on $\mathrm{NiAl}(110)$ at $\mathrm{T}=130 \mathrm{~K}$, and the corresponding fitting curve, which is used in the following temperature-dependent KMC simulation. (b) KMC simulation of island density versus time during deposition. (c) A comparison between the simulated and experimental island density as a function of time and temperature. Uncertainties in simulated values are $\pm 12 \%$ at $130 \mathrm{~K}$, increasing to $\pm 18 \%$ at $175 \mathrm{~K}$. Uncertainties in experimental values are shown. See the text for discussion. (d), (e), and (f) are the STM images at $\mathrm{t}=12 \mathrm{~min}, 57 \mathrm{~min}$, 
and 66 min corresponding to T=130 K, $168 \mathrm{~K}$, and $175 \mathrm{~K}$, respectively. (g) The KMC image just after the deposition. Light gray (white) denotes first (second) layer of Ag. (h), (i), and (j) are the KMC images corresponding to (d), (e), and (f), respectively. The KMC energetic parameters are the same as those of Fig. 7. The size of any image is $27 \times 19 \mathrm{~nm}^{2}$.

Fig. 11. KMC simulated island density $\mathrm{N}_{\mathrm{isl}}$ as a function of coverage $\theta$ or time $\mathrm{t}$ during deposition at $\mathrm{T}=130 \mathrm{~K}$ for three different fluxes and subsequent to deposition also at $\mathrm{T}=130 \mathrm{~K}$. Energetic parameters are the same as those of Fig. 7. (a) $\mathrm{N}_{\text {isl }}$ versus $\theta$ during the deposition. The total Ag coverage is $0.1 \mathrm{ML}$. (b) $\mathrm{N}_{\text {isl }}$ versus $\mathrm{t}$ after the deposition.

Fig. 12. (a) Ag(110) monolayer, (b) Ag doublechain monolayer, and (c) Ag square-hex monolayer structures on $\mathrm{NiAl}(110)$.

Fig. 13. A defect on $\mathrm{NiAl}(110)$ surface formed by replacing a surface $\mathrm{Al}$ atom with a $\mathrm{Ni}$ atom.

Fig. 14. Island density $\mathrm{N}_{\text {isl }}$ versus temperature T. Comparison of KMC simulation results for irreversible ( $\mathrm{i}=1)$ and reversible homogeneous island formation (the latter from the model of Sec. 5), the mean-field rate equation model (RE1 and RE2, see text) for defect-enhanced nucleation with parameters described in the text, and experimental data. The inset (upper left) shows an STM image $\left(100 \times 100 \mathrm{~nm}^{2}\right)$ of surface point defects observed after one standard sample preparation procedure. Features appear similar to point defects observed by STM on $\mathrm{Au} 3 \mathrm{Cu}(001)$ (Ref. 45) and by another group on $\mathrm{NiAl}(110)$ (Ref. 43). 
Figures

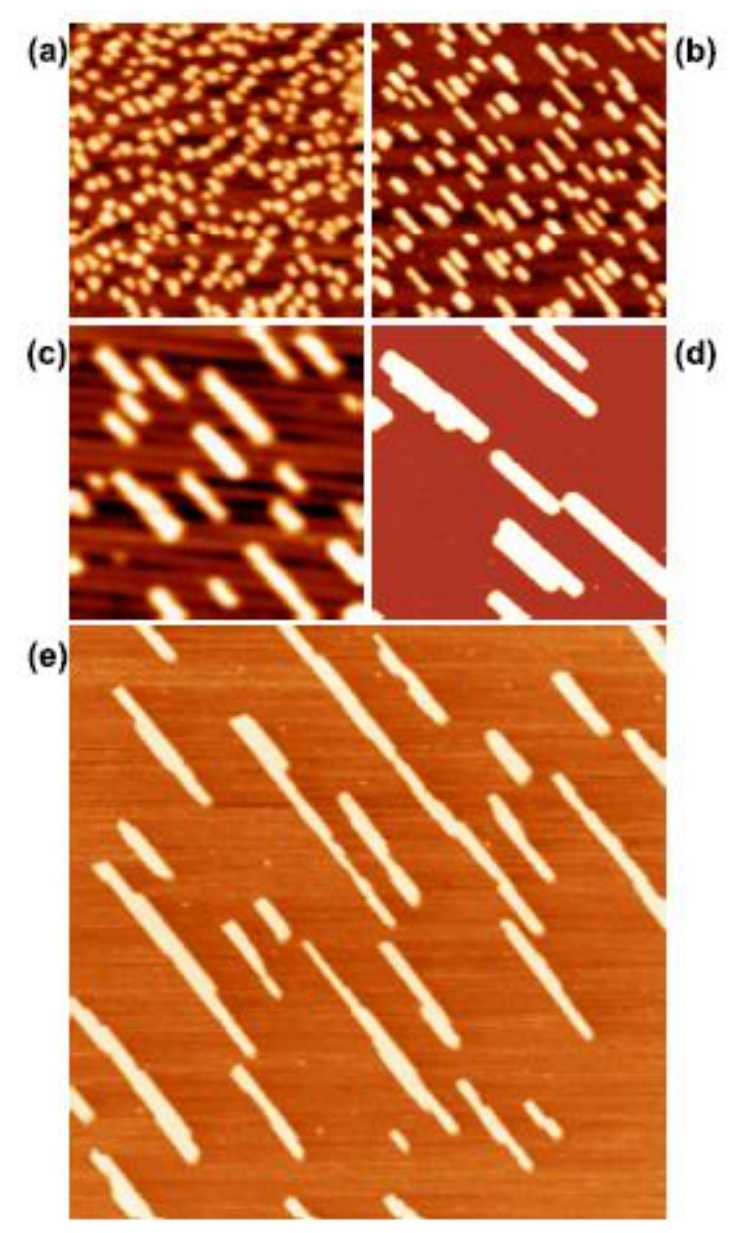

Fig. 1 


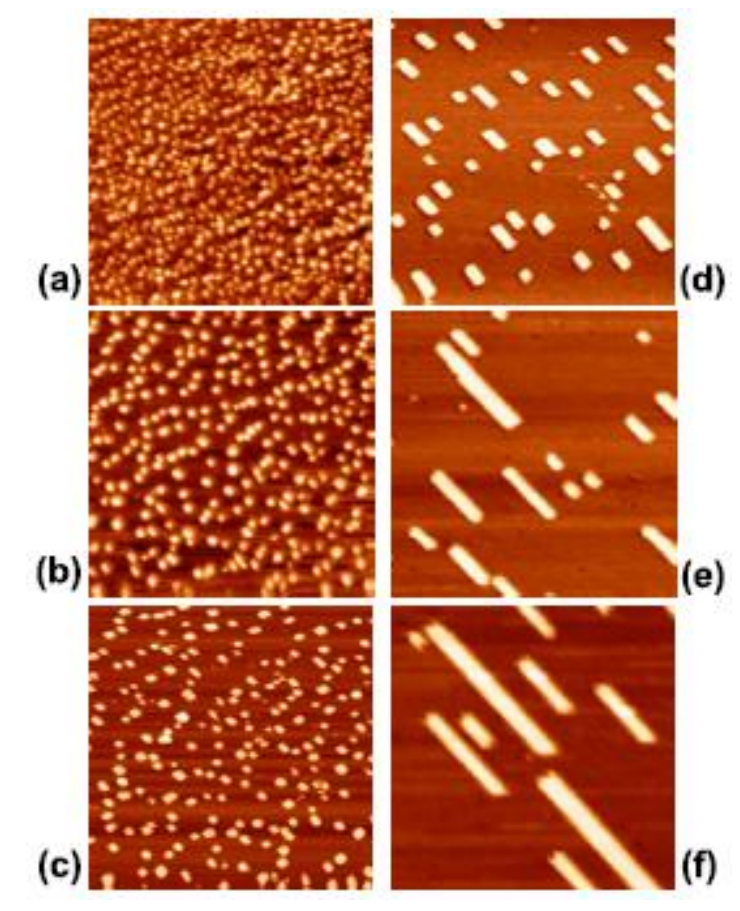

Fig. 2 


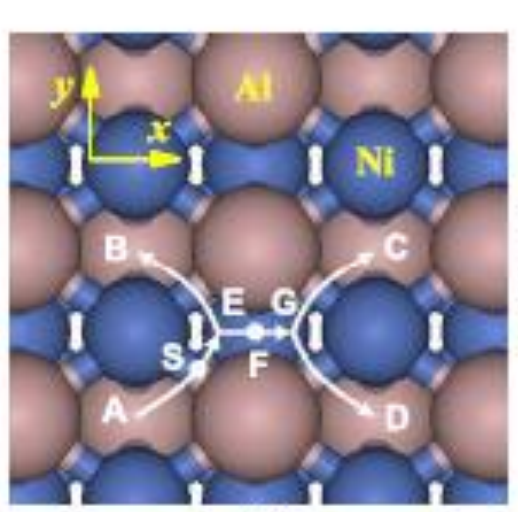

(a)

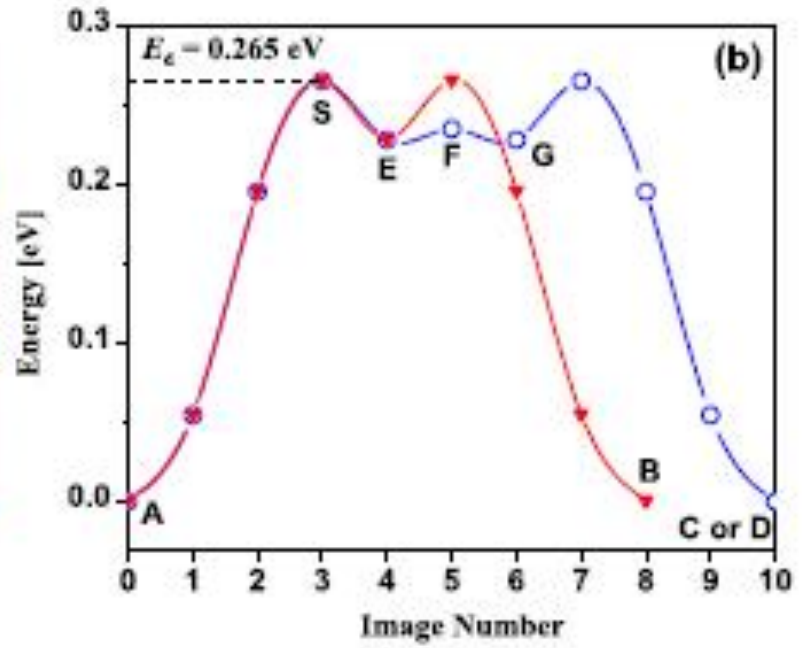

Fig. 3 


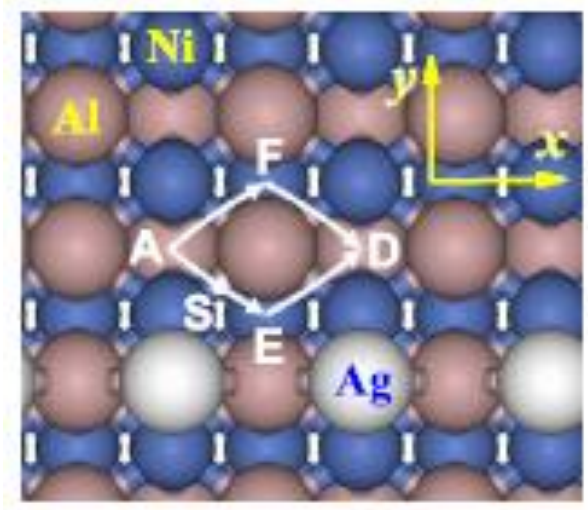

(a)

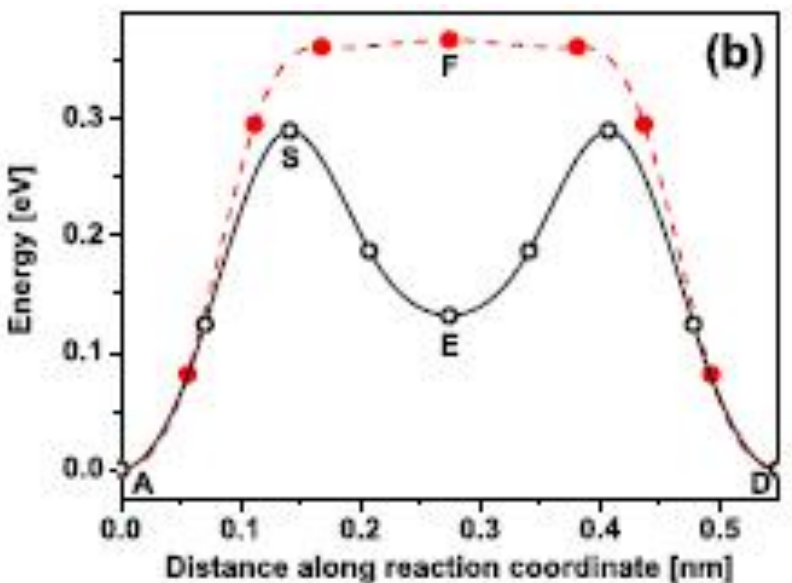

Fig. 4 


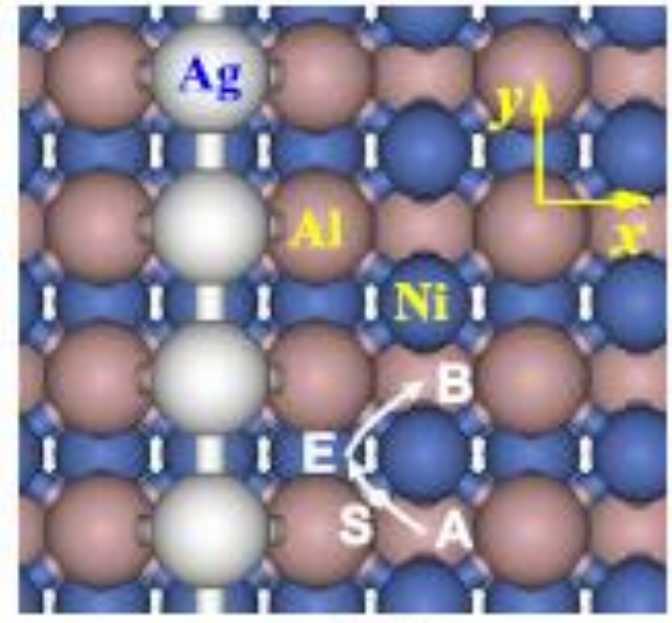

(a)

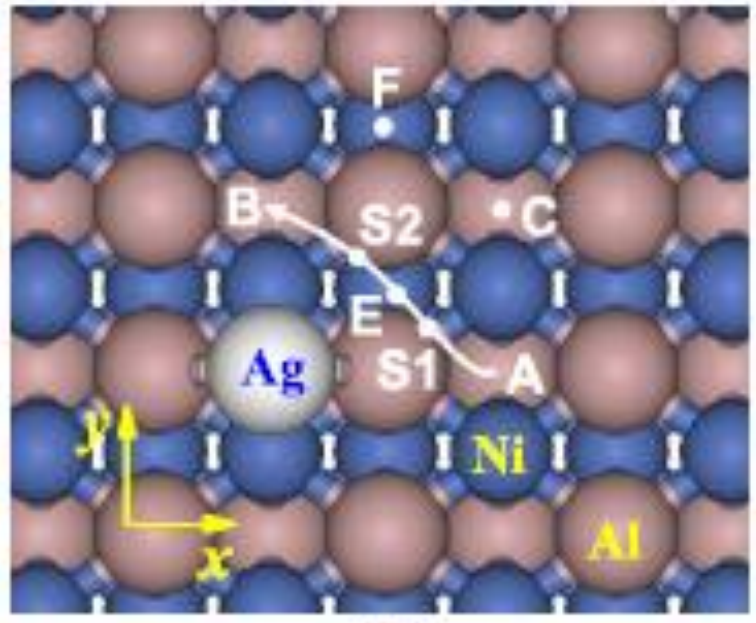

(b)

Fig. 5 


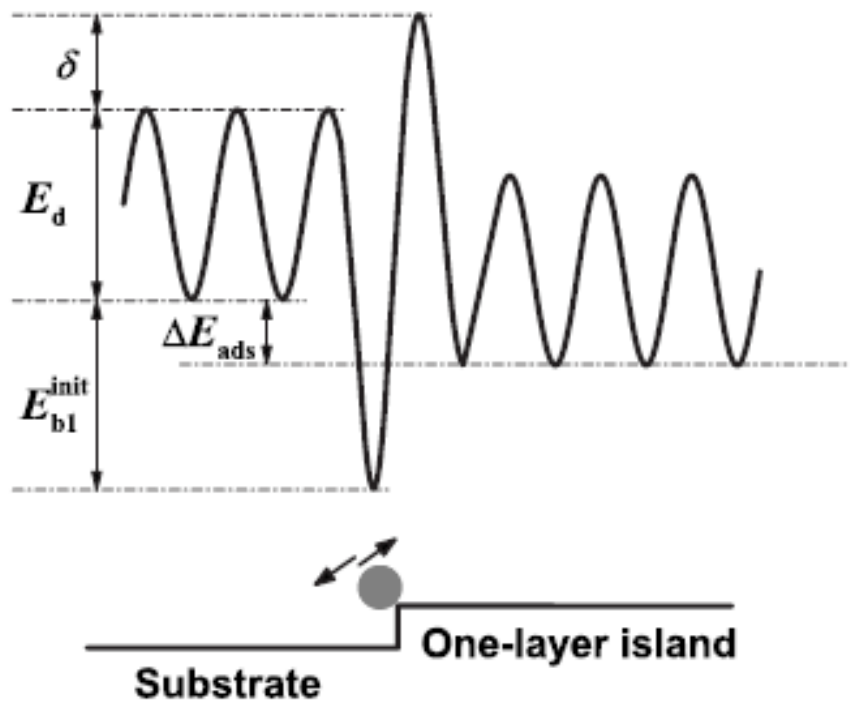

Fig. 6 


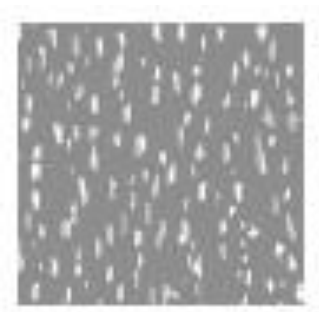

a
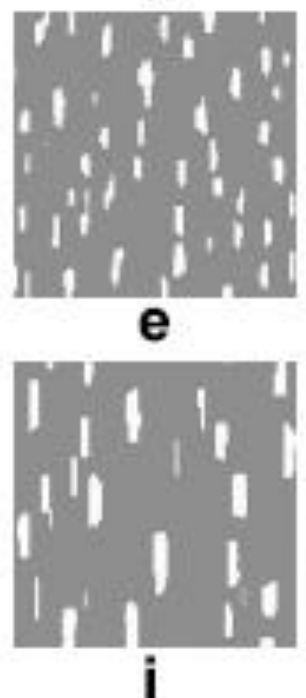

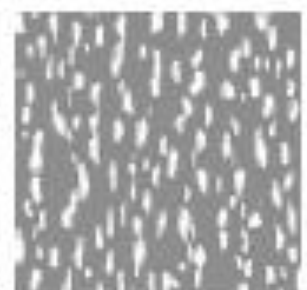

b
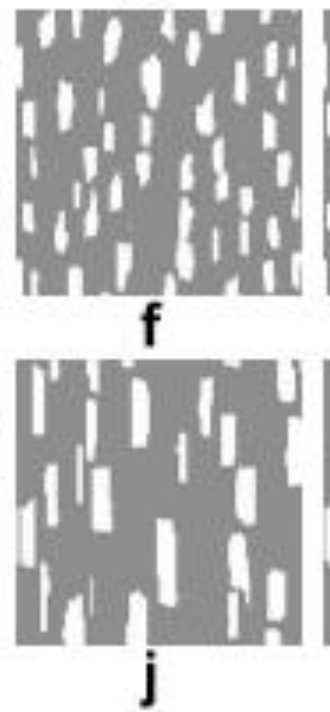

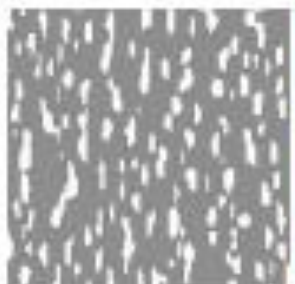

C

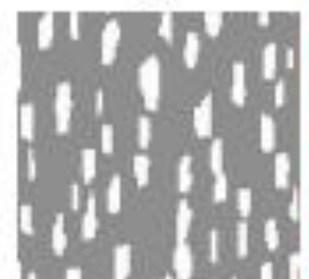

g

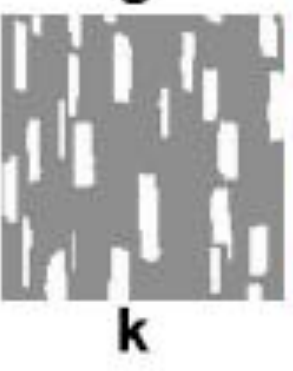

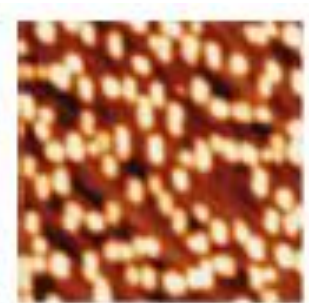

d
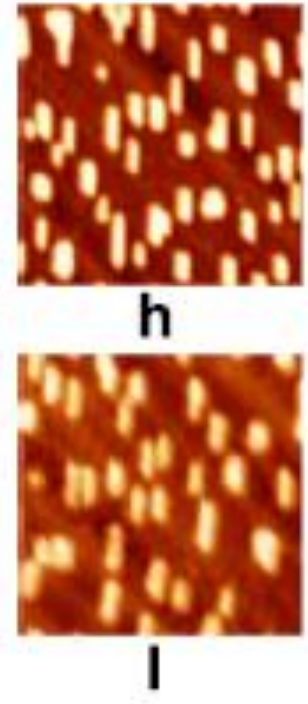

Fig. 7 


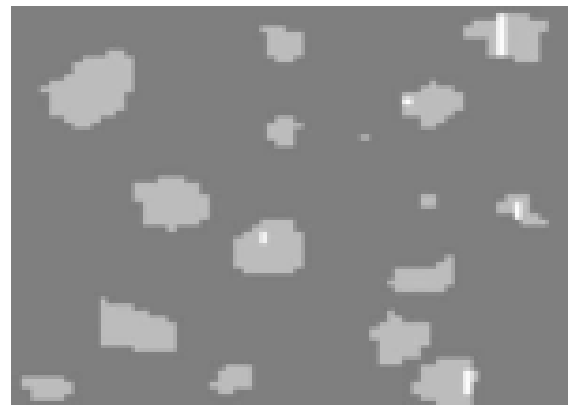

(a)

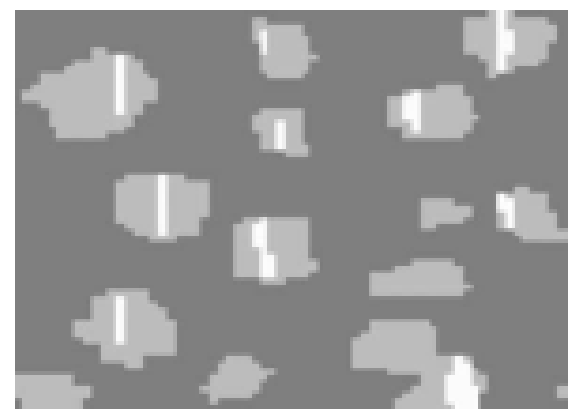

(b)

Fig. 8 


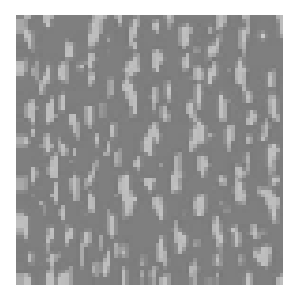

a

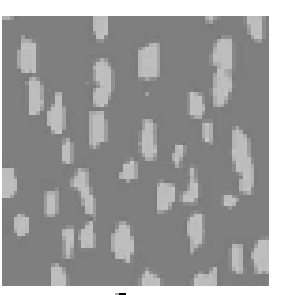

b

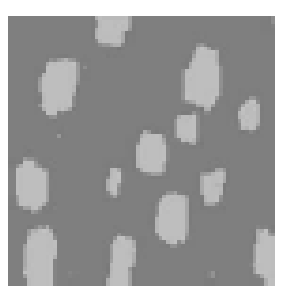

C

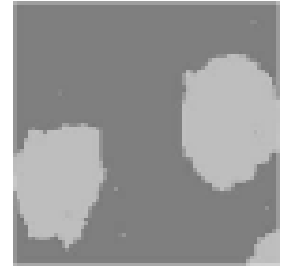

d

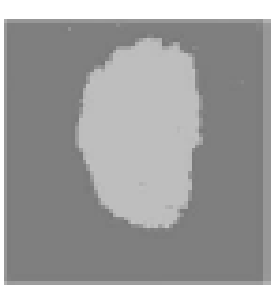

e

Fig. 9 


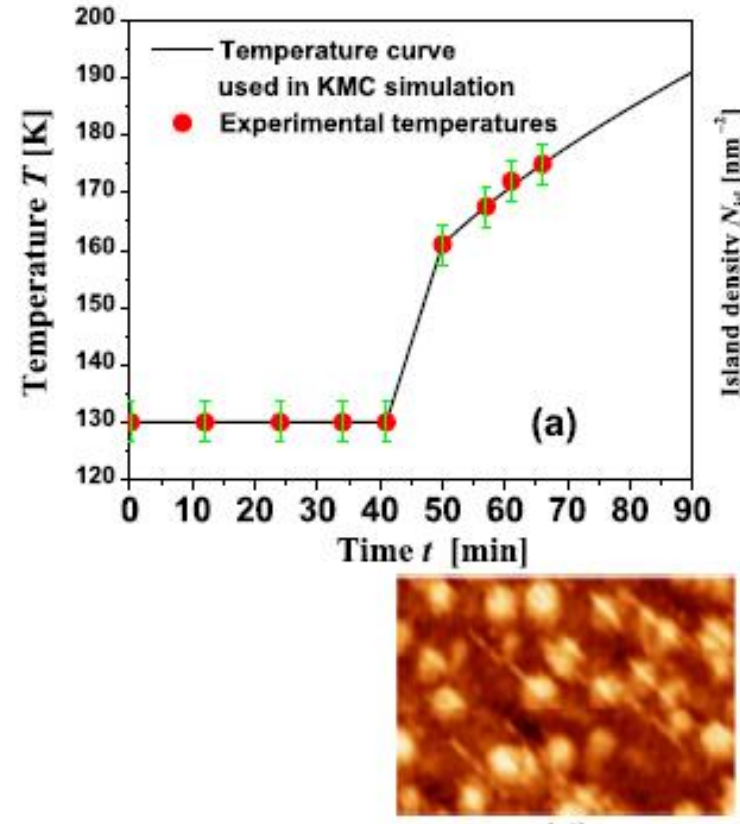

(d)

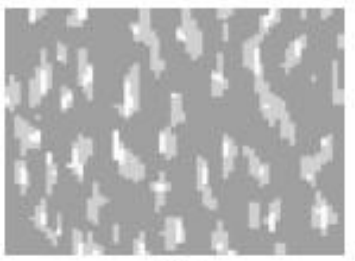

(g)

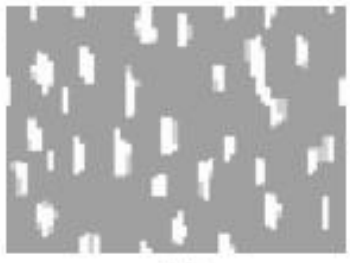

(h)

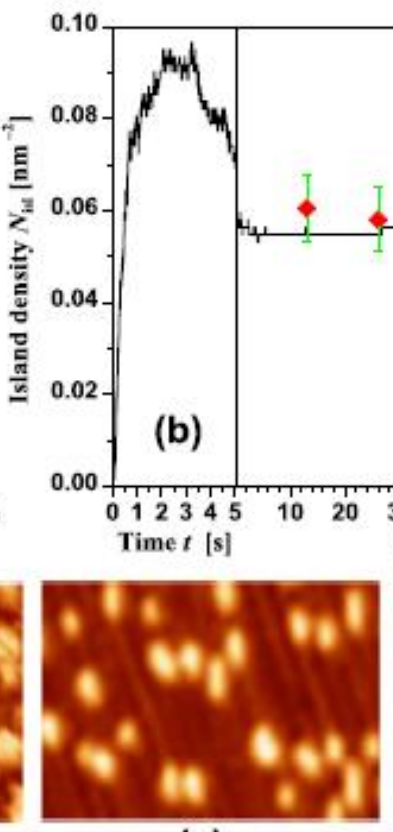

(e)

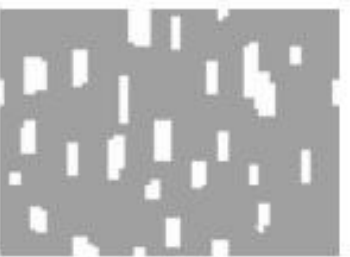

(i)

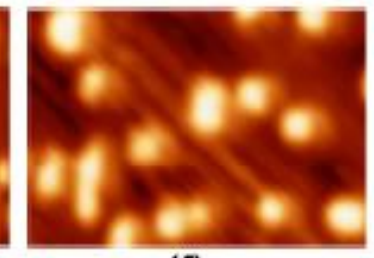

(f)

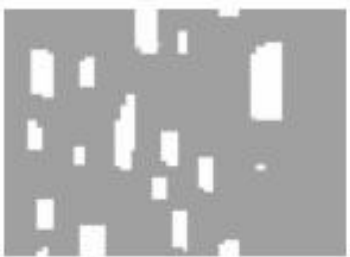

(j)

Fig. 10 


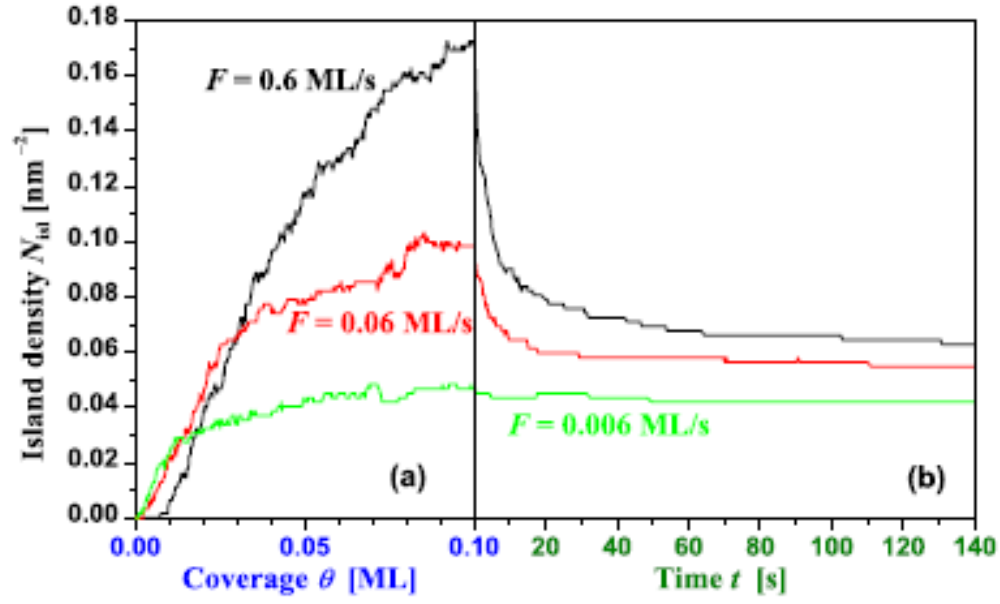

Fig. 11 


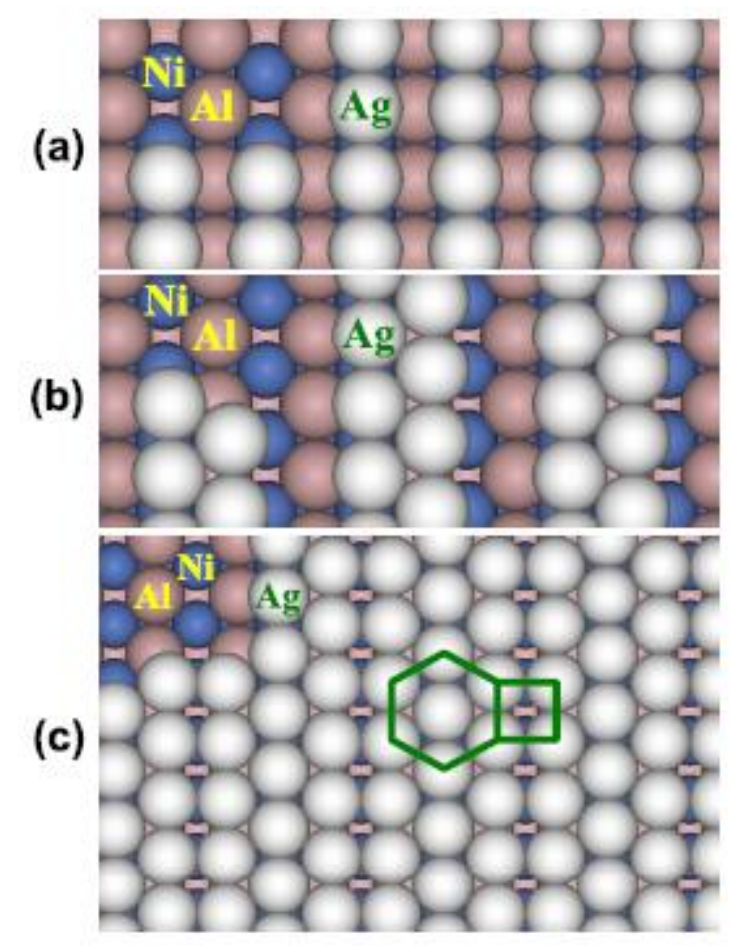

Fig. 12 


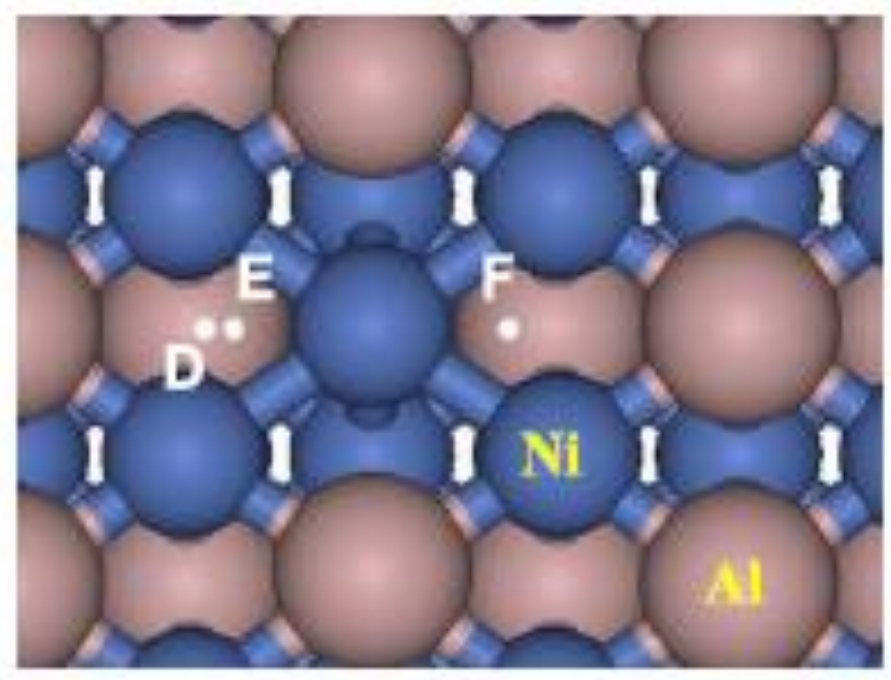

Fig. 13 


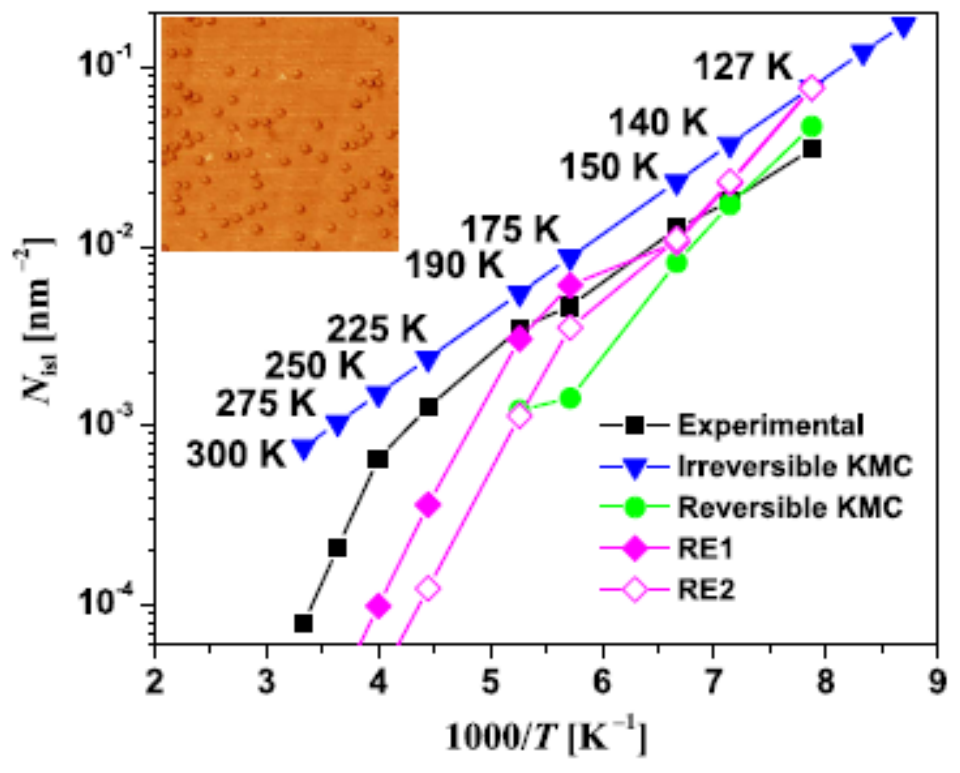

Fig. 14 


\section{APPENDIX III. EXPERIMENTAL DATABASE}

\section{Tables:}

1. Table 1: Detailed STM experiment database

2. Table 2: Detailed LEED experiment database 
Table 1

\begin{tabular}{|c|c|c|}
\hline Folder name ${ }^{a}$ & Experiment description & Notes ${ }^{b}$ \\
\hline 2006_12_07 & $\begin{array}{l}\text { Ag deposition on } \mathrm{Zr}-\mathrm{Cu}-\mathrm{Ni}-\mathrm{Al} \text { at } 300 \mathrm{~K} \\
\text { BMG sample \#1 (DJS-20-137-\#1) } \\
\text { Flux }=6.7 \times 10^{-3} \mathrm{ML} / \mathrm{s} \\
\text { Coverage up to } 2.4 \mathrm{ML}\end{array}$ & FQ\#2 Pg. 66 \\
\hline 2006_12_08 & $\begin{array}{l}\text { Ag deposition on } \mathrm{Zr}-\mathrm{Cu}-\mathrm{Ni}-\mathrm{Al} \text { at } 300 \mathrm{~K} \\
\text { BMG sample \#1 } \\
\text { Flux }=6.7 \times 10^{-3} \mathrm{ML} / \mathrm{s} \\
\text { Coverage up to } 2.4 \mathrm{ML}\end{array}$ & FQ\#2 Pg. 71 \\
\hline 2006_12_09 & $\begin{array}{l}\text { Ag deposition on } \mathrm{Zr}-\mathrm{Cu}-\mathrm{Ni}-\mathrm{Al} \text { at } 300 \mathrm{~K} \\
\text { BMG sample \#1 } \\
\text { Flux }=6.7 \times 10^{-3} \mathrm{ML} / \mathrm{s} \\
\text { Coverage up to } 1.2 \mathrm{ML}\end{array}$ & FQ\#2 Pg 76 \\
\hline 2006_12_11 & $\begin{array}{l}\text { Ag deposition on } \mathrm{Zr}-\mathrm{Cu}-\mathrm{Ni}-\mathrm{Al} \text { at } 300 \mathrm{~K} \\
\text { BMG sample \#1 } \\
\text { Flux }=6.7 \times 10^{-3} \mathrm{ML} / \mathrm{s} \\
\text { Coverage up to } 11.6 \mathrm{ML}\end{array}$ & FQ\#2 Pg 79 \\
\hline 2007_06_30 & $\begin{array}{l}\text { Ag deposition on } \mathrm{Zr}-\mathrm{Cu}-\mathrm{Ni}-\mathrm{Al} \text { at } 300 \mathrm{~K} \\
\text { BMG sample \#1 } \\
\text { Flux }=6.7 \times 10^{-3} \mathrm{ML} / \mathrm{s} \\
\text { Coverage up to } 8 \mathrm{ML}\end{array}$ & BU\#4 Pg. 69 \\
\hline 2007_07_01 & $\begin{array}{l}\text { Ag deposition on } \mathrm{Zr}-\mathrm{Cu}-\mathrm{Ni}-\mathrm{Al} \text { at } 300 \mathrm{~K} \\
\text { BMG sample \#1 } \\
\text { Flux }=6.7 \times 10^{-3} \mathrm{ML} / \mathrm{s} \\
\text { Coverage up to } 22 \mathrm{ML}\end{array}$ & BU\#4 Pg. 71 \\
\hline 2007_07_07 & $\begin{array}{l}\text { Clean surface of } \mathrm{Zr}-\mathrm{Cu}-\mathrm{Ni}-\mathrm{Al} \text { at } 300 \mathrm{~K} \\
\text { BMG sample \#1 }\end{array}$ & BU\#4 Pg. 79 \\
\hline 2007_07_08 & $\begin{array}{l}\text { Ag deposition on } \mathrm{Zr}-\mathrm{Cu}-\mathrm{Ni}-\mathrm{Al} \text { at } 300 \mathrm{~K} \\
\text { BMG sample \#1 } \\
\text { Flux }=6.7 \times 10^{-3} \mathrm{ML} / \mathrm{s} \\
\text { Coverage up to } 14 \mathrm{ML}\end{array}$ & BU\#4 Pg. 81 \\
\hline 2007_08_06 & $\begin{array}{l}\text { Clean surface of } \mathrm{Zr}-\mathrm{Cu}-\mathrm{Ni}-\mathrm{Al} \text { at } 300 \mathrm{~K} \\
\text { BMG sample \# } 1\end{array}$ & BU\#4 Pg. 91 \\
\hline 2007_08_10 & $\begin{array}{l}\text { Ag deposition on } \mathrm{Zr}-\mathrm{Cu}-\mathrm{Ni}-\mathrm{Al} \text { at } 300 \mathrm{~K} \\
\text { BMG sample \#1 } \\
\text { Flux }=6.7 \times 10^{-3} \mathrm{ML} / \mathrm{s} \\
\text { Coverage up to } 14 \mathrm{ML}\end{array}$ & BU\#4 Pg. 97 \\
\hline
\end{tabular}

Table 1 


\begin{tabular}{|c|c|c|}
\hline Folder name & Experiment description & Notes \\
\hline 2007_08_12 & $\begin{array}{l}\text { Ag deposition on } \mathrm{Zr}-\mathrm{Cu}-\mathrm{Ni}-\mathrm{Al} \text { at } 300 \mathrm{~K} \\
\text { BMG sample \#1 } \\
\text { Flux }=6.7 \times 10^{-3} \mathrm{ML} / \mathrm{s} \\
\text { Coverage up to } 14 \mathrm{ML}\end{array}$ & BU\#4 Pg. 99 \\
\hline 2007_08_16 & $\begin{array}{l}\text { Ag deposition on } \mathrm{Zr}-\mathrm{Cu}-\mathrm{Ni}-\mathrm{Al} \text { at } 300 \mathrm{~K} \\
\text { BMG sample \#2 (DJS-20-137-\#2) } \\
\text { Flux }=6.7 \times 10^{-3} \mathrm{ML} / \mathrm{s} \\
\text { Coverage up to } 20 \mathrm{ML}\end{array}$ & BU\#4 Pg. 107 \\
\hline 2007_08_19 & $\begin{array}{l}\text { Ag deposition on } \mathrm{Zr}-\mathrm{Cu}-\mathrm{Ni}-\mathrm{Al} \text { at } 300 \mathrm{~K} \\
\text { BMG sample } \# 2 \\
\text { Flux }=6.7 \times 10^{-3} \mathrm{ML} / \mathrm{s} \\
\text { Coverage up to } 20 \mathrm{ML}\end{array}$ & BU\#4 Pg. 111 \\
\hline 2007_08_20 & $\begin{array}{l}\text { Ag deposition on } \mathrm{Zr}-\mathrm{Cu}-\mathrm{Ni}-\mathrm{Al} \text { at } 190 \mathrm{~K} \\
\text { BMG sample } \# 2 \\
\text { Flux }=6.7 \times 10^{-3} \mathrm{ML} / \mathrm{s} \\
\text { Coverage up to } 20 \mathrm{ML}\end{array}$ & BU\#4 Pg. 116 \\
\hline 2007_08_21 & $\begin{array}{l}\text { Ag deposition on } \mathrm{Zr}-\mathrm{Cu}-\mathrm{Ni}-\mathrm{Al} \text { at } 240 \mathrm{~K} \\
\text { BMG sample } \# 2 \\
\text { Flux }=6.7 \times 10^{-3} \mathrm{ML} / \mathrm{s} \\
\text { Coverage up to } 20 \mathrm{ML}\end{array}$ & BU\#4 Pg. 120 \\
\hline 2007_08_24 & $\begin{array}{l}\text { Ag deposition on } \mathrm{Zr}-\mathrm{Cu}-\mathrm{Ni}-\mathrm{Al} \text { at } 260 \mathrm{~K} \\
\text { BMG sample } \# 2 \\
\text { Flux }=6.7 \times 10^{-3} \mathrm{ML} / \mathrm{s} \\
\text { Coverage up to } 20 \mathrm{ML}\end{array}$ & BU\#4 Pg. 124 \\
\hline 2007_08_26 & $\begin{array}{l}\text { Ag deposition on } \mathrm{Zr}-\mathrm{Cu}-\mathrm{Ni}-\mathrm{Al} \text { at } 300 \mathrm{~K} \\
\text { BMG sample } \# 2 \\
\text { Flux }=6.7 \times 10^{-3} \mathrm{ML} / \mathrm{s} \\
\text { Coverage up to } 20 \mathrm{ML}\end{array}$ & BU\#4 Pg. 129 \\
\hline 2007_08_27 & $\begin{array}{l}\text { Ag deposition on } \mathrm{Zr}-\mathrm{Cu}-\mathrm{Ni}-\mathrm{Al} \text { at } 300 \mathrm{~K} \\
\text { BMG sample } \# 2 \\
\text { Flux }=6.7 \times 10^{-3} \mathrm{ML} / \mathrm{s} \\
\text { Coverage up to } 20 \mathrm{ML}\end{array}$ & BU\#4 Pg. 135 \\
\hline 2007_08_29 & $\begin{array}{l}\text { Ag deposition on } \mathrm{Zr}-\mathrm{Cu}-\mathrm{Ni}-\mathrm{Al} \text { at } 300 \mathrm{~K} \\
\text { BMG sample } \# 2 \\
\text { Flux }=6.7 \times 10^{-3} \mathrm{ML} / \mathrm{s} \\
\text { Coverage up to } 24 \mathrm{ML}\end{array}$ & BU\#4 Pg. 143 \\
\hline
\end{tabular}

Table 1 Continued 


\begin{tabular}{|c|c|c|}
\hline Folder name & Experiment description & Notes \\
\hline 2007_08_30 & $\begin{array}{l}\text { Ag deposition on } \mathrm{Zr}-\mathrm{Cu}-\mathrm{Ni}-\mathrm{Al} \text { at } 300 \mathrm{~K} \\
\mathrm{BMG} \text { sample } \# 2 \\
\text { Flux }=6.7 \times 10^{-3} \mathrm{ML} / \mathrm{s} \\
\text { Coverage up to } 20 \mathrm{ML}\end{array}$ & BU\#4 Pg. 149 \\
\hline 2007_09_22 & Clean surface of $\mathrm{NiAl}(110)$ & BU\#5 Pg. 16 \\
\hline 2007_09_30 & $\begin{array}{l}\mathrm{Ag} \text { deposition on } \mathrm{NiAl}(110) \text { at } 300 \mathrm{~K} \\
\text { Flux }=6.7 \times 10^{-3} \mathrm{ML} / \mathrm{s} \\
\text { Coverage: } 0.4 \mathrm{ML}, 1.2 \mathrm{ML}, 2.4 \mathrm{ML}, 4.4 \mathrm{ML}\end{array}$ & BU\#5 Pg. 23 \\
\hline 2007_10_08 & $\begin{array}{l}\mathrm{Ag} \text { deposition on } \mathrm{NiAl}(110) \text { at } 300 \mathrm{~K} \\
\text { Flux }=6.7 \times 10^{-3} \mathrm{ML} / \mathrm{s} \\
\text { Coverage: } 12 \mathrm{ML}\end{array}$ & BU\#5 Pg. 33 \\
\hline 2007_10_25 & Clean surface of $\mathrm{NiAl}(110)$ & BU\#5 Pg. 46 \\
\hline 2007_10_31 & $\begin{array}{l}\mathrm{Ag} \text { deposition on } \mathrm{NiAl}(110) \text { at } 250 \mathrm{~K} \\
\text { Flux }=6.7 \times 10^{-3} \mathrm{ML} / \mathrm{s} \\
\text { Coverage: } 0.4 \mathrm{ML}\end{array}$ & BU\#5 Pg. 51 \\
\hline 2007_11_08 & Clean surface of NiAl(110) & BU\#5 Pg. 56 \\
\hline 2007_11_13 & $\begin{array}{l}\text { Ag deposition on } \mathrm{NiAl}(110) \text { at } 140 \mathrm{~K} \\
\text { Flux }=6.7 \times 10^{-3} \mathrm{ML} / \mathrm{s} \\
\text { Coverage: } 0.4 \mathrm{ML}\end{array}$ & BU\#5 Pg. 59 \\
\hline 2007_12_03 & $\begin{array}{l}\mathrm{Ag} \text { deposition on } \mathrm{NiAl}(110) \text { at } 130 \mathrm{~K} \\
\text { Flux }=5.6 \times 10^{-2} \mathrm{ML} / \mathrm{s} \\
\text { Coverage: } 0.3 \mathrm{ML}\end{array}$ & BU\#5 Pg. 69 \\
\hline 2007_12_09 & $\begin{array}{l}\mathrm{Ag} \text { deposition on } \mathrm{NiAl}(110) \text { at } 140 \mathrm{~K} \\
\text { Flux }=5.6 \times 10^{-2} \mathrm{ML} / \mathrm{s} \\
\text { Coverage: } 0.3 \mathrm{ML}\end{array}$ & BU\#5 Pg. 75 \\
\hline 2007_12_14 & $\begin{array}{l}\text { Ag deposition on } \mathrm{NiAl}(110) \text { at } 140 \mathrm{~K} \\
\text { Flux }=5.6 \times 10^{-2} \mathrm{ML} / \mathrm{s} \\
\text { Coverage: } 0.3 \mathrm{ML}\end{array}$ & BU\#5 Pg. 83 \\
\hline 2007_12_23 & Clean surface of $\mathrm{NiAl}(110)$ & BU\#5 Pg. 95 \\
\hline 2007_12_28 & Clean surface of $\mathrm{NiAl}(110)$ & BU\#5 Pg. 102 \\
\hline 2008_01_15 & $\begin{array}{l}\mathrm{Ag} \text { deposition on } \mathrm{NiAl}(110) \text { at } 140 \mathrm{~K} \\
\text { Flux }=5.6 \times 10^{-2} \mathrm{ML} / \mathrm{s} \\
\text { Coverage: } 0.3 \mathrm{ML}\end{array}$ & BU\#5 Pg. 127 \\
\hline
\end{tabular}




\begin{tabular}{|c|c|c|}
\hline Folder name & Experiment description & Notes \\
\hline 2008_01_18 & $\begin{array}{l}\mathrm{Ag} \text { deposition on } \mathrm{NiAl}(110) \text { at } 260 \mathrm{~K} \\
\text { Flux }=6.7 \times 10^{-3} \mathrm{ML} / \mathrm{s} \\
\text { Coverage: up to } 20 \mathrm{ML}\end{array}$ & BU\#5 Pg. 132 \\
\hline 2008_01_24 & $\begin{array}{l}\mathrm{Ag} \text { deposition on } \mathrm{NiAl}(110) \text { at } 180 \mathrm{~K} \\
\text { Flux }=6.7 \times 10^{-3} \mathrm{ML} / \mathrm{s} \\
\text { Coverage: } 0.4 \mathrm{ML}\end{array}$ & BU\#5 Pg. 141 \\
\hline 2008_02_04 & $\begin{array}{l}\mathrm{Ag} \text { deposition on } \mathrm{NiAl}(110) \text { at } 180 \mathrm{~K} \\
\text { Flux }=6.7 \times 10^{-3} \mathrm{ML} / \mathrm{s} \\
\text { Coverage: up to } 20 \mathrm{ML}\end{array}$ & BU\#5 Pg. 146 \\
\hline 2008_02_07 & Clean surface of $\mathrm{NiAl}(110)$ & BU\#5 Pg. 151 \\
\hline 2008_02_09 & $\begin{array}{l}\mathrm{Ag} \text { deposition on } \mathrm{NiAl}(110) \text { at } 260 \mathrm{~K} \\
\text { Flux }=6.7 \times 10^{-3} \mathrm{ML} / \mathrm{s} \\
\text { Coverage: } \mathrm{Up} \text { to } 20 \mathrm{ML}\end{array}$ & BU\#5 Pg. 152 \\
\hline 2008_03_17 & Dirty surface of $\mathrm{NiAl}(110)$ (as-loaded) & BU\#5 Pg. 193 \\
\hline 2008_03_19 & Clean surface of $\mathrm{NiAl}(110)$ & BU\#5 Pg. 197 \\
\hline 2008_03_20 & Sputtered clean surface of $\mathrm{NiAl}(110)$ & BU\#5 Pg. 197 \\
\hline 2008_03_21 & Sputtered clean surface of $\mathrm{NiAl}(110)$ & BU\#5 Pg. 198 \\
\hline 2008_04_23 & $\begin{array}{l}\text { Oxidized } \mathrm{NiAl}(110) \text { surface } \\
1200 \text { L oxygen dose }\end{array}$ & BU\#5 Pg. 219 \\
\hline 2008_07_22 & \begin{tabular}{|l} 
Failed $\mathrm{Ag}$ deposition on $\mathrm{NiAl}(110)$ experiment \\
\end{tabular} & BU\#5 Pg. 252 \\
\hline 2008_07_25 & $\begin{array}{l}\text { Ag deposition on oxidized NiAl(110) at } 300 \mathrm{~K} \\
2000 \text { L oxygen dose } \\
\text { Flux: Not calibrated }\end{array}$ & BU\#5 Pg. 255 \\
\hline 2008_08_01 & $\begin{array}{l}\text { Oxidized NiAl(110) surface } \\
1850 \text { L oxygen dose (three times) }\end{array}$ & BU\#5 Pg. 259 \\
\hline 2008_09_25 & $\begin{array}{l}\text { Ag deposition on oxidized } \mathrm{NiAl}(110) \text { at } 300 \mathrm{~K} \\
1800 \mathrm{~K} \text { oxygen dose } \\
\text { Flux: Not calibrated }\end{array}$ & BU\#5 Pg. 290 \\
\hline 2008_12_27 & $\begin{array}{l}\text { Ni deposition on NiAl(110) at } 300 \mathrm{~K} \\
\text { Flux: } 3.3 \times 10^{-4} \mathrm{ML} / \mathrm{s} \\
\text { Coverage: } 0.02 \mathrm{ML}\end{array}$ & DJ\#3 Pg. 35 \\
\hline 2008_12_29 & $\begin{array}{l}\text { Ni deposition on } \mathrm{NiAl}(110) \text { at } 300 \mathrm{~K} \\
\text { Flux: } 3.3 \times 10^{-4} \mathrm{ML} / \mathrm{s} \\
\text { Coverage: Up to } 0.9 \mathrm{ML}\end{array}$ & DJ\#3 Pg. 37 \\
\hline
\end{tabular}

Table 1 Continued 


\begin{tabular}{|c|c|c|}
\hline Folder name & Experiment description & Notes \\
\hline 2009_01_01 & $\begin{array}{l}\text { Ni deposition on } \mathrm{NiAl}(110) \text { at } 300 \mathrm{~K} \\
\text { Flux: } 3.1 \times 10^{-3} \mathrm{ML} / \mathrm{s} \\
\text { Coverage: } \mathrm{Up} \text { to } 0.2 \mathrm{ML}\end{array}$ & DJ\#3 Pg. 39 \\
\hline 2009_01_05 & $\begin{array}{l}\text { Ni deposition on } \mathrm{NiAl}(110) \text { at } 300 \mathrm{~K} \\
\text { Flux: } 3.1 \times 10^{-3} \mathrm{ML} / \mathrm{s} \\
\text { Coverage: Up to } 0.74 \mathrm{ML}\end{array}$ & DJ\#3 Pg. 43 \\
\hline 2009_01_08 & $\begin{array}{l}\text { Ni deposition on } \mathrm{NiAl}(110) \text { at } 300 \mathrm{~K} \\
\text { Flux: } 3.3 \times 10^{-3} \mathrm{ML} / \mathrm{s} \\
\text { Coverage: } \mathrm{Up} \text { to } 0.2 \mathrm{ML} \\
\text { Ag co-deposition after Ni deposition } \\
\text { (determination of substrate orientation) }\end{array}$ & DJ\#3 Pg. 46 \\
\hline 2009_01_13 & $\begin{array}{l}\text { Al, Ni sequential co-deposition on } \mathrm{NiAl}(110) \text { at } \\
300 \mathrm{~K} \\
\text { Al flux: } 3.3 \times 10^{-3} \mathrm{ML} / \mathrm{s} \\
\text { Al coverage: } 0.1 \mathrm{ML} \\
\text { Ni flux: } 3.3 \times 10^{-3} \mathrm{ML} / \mathrm{s} \\
\text { Ni coverage: } 0.1 \mathrm{ML} \\
\end{array}$ & DJ\#3 Pg. 50 \\
\hline 2009_01_17 & $\begin{array}{l}\text { Al, Ni, Ag sequential co-deposition on } \mathrm{NiAl}(110) \\
\text { at } 300 \mathrm{~K} \\
\text { Al flux: } 6.7 \times 10^{-3} \mathrm{ML} / \mathrm{s} \\
\text { Al coverage: } 0.2 \mathrm{ML} \\
\mathrm{Ni} \text { flux: } 3.3 \times 10^{-3} \mathrm{ML} / \mathrm{s} \\
\mathrm{Ni} \text { coverage: } 0.235 \mathrm{ML} \\
\text { Ag deposition to check substrate surface orientation }\end{array}$ & DJ\#3 Pg. 53 \\
\hline 2009_01_13 & $\begin{array}{l}\mathrm{Ni}, \mathrm{Al} \text { sequential co-deposition on } \mathrm{NiAl}(110) \text { at } \\
300 \mathrm{~K} \\
\mathrm{Ni} \text { flux: } 1.7 \times 10^{-3} \mathrm{ML} / \mathrm{s} \\
\mathrm{Ni} \text { coverage: } 0.15 \mathrm{ML} \\
\text { Al flux: } 3.3 \times 10^{-3} \mathrm{ML} / \mathrm{s} \\
\text { Al coverage: } 0.22 \mathrm{ML}\end{array}$ & DJ\#3 Pg. 55 \\
\hline 2009_03_08 & Clean surface of $\mathrm{NiAl}(110)$ & DJ\#3 Pg. 66 \\
\hline 2009_03_12 & $\begin{array}{l}\text { Ni deposition on NiAl(110) at } 400 \mathrm{~K} \\
\text { Flux: } 1.2 \times 10^{-3} \mathrm{ML} / \mathrm{s} \\
\text { Coverage: } \mathrm{Up} \text { to } 0.18 \mathrm{ML} \\
\text { Subsequent anneal to } 450 \mathrm{~K} \text { and } 500 \mathrm{~K}\end{array}$ & DJ\#3 Pg. 68 \\
\hline
\end{tabular}

Table 1 Continued 


\begin{tabular}{|c|c|c|}
\hline Folder name & Experiment description & Notes \\
\hline 2009_03_15 & $\begin{array}{l}\text { Ni deposition on } \mathrm{NiAl}(110) \text { at } 200 \mathrm{~K} \\
\text { Flux: } 1.3 \times 10^{-3} \mathrm{ML} / \mathrm{s} \\
\text { Coverage: } 0.2 \mathrm{ML} \\
\text { Subsequent anneal to } 300 \mathrm{~K}, 400 \mathrm{~K} \text { and } 500 \mathrm{~K}\end{array}$ & DJ\#3 Pg. 70 \\
\hline 2009_03_22 & $\begin{array}{l}\mathrm{Ni}, \mathrm{Al} \text { sequential co-deposition on NiAl(110) at } \\
300 \mathrm{~K} \\
\mathrm{Ni} \text { flux: } 1.1 \times 10^{-3} \mathrm{ML} / \mathrm{s} \\
\mathrm{Ni} \text { coverage: } 0.22 \mathrm{ML} \\
\text { Al flux: } 3.5 \times 10^{-3} \mathrm{ML} / \mathrm{s} \\
\text { Al coverage: } 0.16 \mathrm{ML} \\
\text { Subsequent anneal to } 400 \mathrm{~K} \text { and } 500 \mathrm{~K}\end{array}$ & DJ\#3 Pg. 76 \\
\hline 2009_03_25 & $\begin{array}{l}\text { Al, Ni sequential co-deposition on NiAl(110) at } \\
300 \mathrm{~K} \\
\text { Al flux: } 1.9 \times 10^{-3} \mathrm{ML} / \mathrm{s} \\
\text { Al coverage: } 0.14 \mathrm{ML} \\
\text { Ni flux: } 1.1 \times 10^{-3} \mathrm{ML} / \mathrm{s} \\
\text { Ni coverage: } 0.22 \mathrm{ML} \\
\text { Subsequent anneal to } 400 \mathrm{~K} \text { and } 500 \mathrm{~K}\end{array}$ & DJ\#3 Pg. 78 \\
\hline 2009_03_28 & $\begin{array}{l}\text { Al deposition on } \mathrm{NiAl}(110) \text { at } 300 \mathrm{~K} \\
\text { Flux: } 3 \times 10^{-3} \mathrm{ML} / \mathrm{s} \\
\text { Coverage: } \mathrm{Up} \text { to } 1.8 \mathrm{ML}\end{array}$ & DJ\#3 Pg. 80 \\
\hline 2009_03_31 & $\begin{array}{l}\text { Ni deposition on } \mathrm{NiAl}(110) \text { at } 300 \mathrm{~K} \\
\text { Flux: } 1.1 \times 10^{-3} \mathrm{ML} / \mathrm{s} \\
\text { Coverage: Up to } 10.6 \mathrm{ML}\end{array}$ & DJ\#3 Pg. 82 \\
\hline 2009_09_24 & $\begin{array}{l}\text { Ag deposition on } \mathrm{NiAl}(110) \text { at } 200 \mathrm{~K} \\
\text { Flux: Not calibrated }\end{array}$ & DJ\#3 Pg. 104 \\
\hline 2009_10_02 & $\begin{array}{l}\text { Ag deposition on } \mathrm{NiAl}(110) \text { at } 300 \mathrm{~K} \\
\text { Flux: Not calibrated }\end{array}$ & DJ\#3 Pg. 110 \\
\hline 2009_10_11 & $\begin{array}{l}\text { Ag deposition on } \mathrm{NiAl}(110) \text { at } 225 \mathrm{~K} \\
\text { Flux: Not calibrated } \\
\text { Coverage: } 0.3 \mathrm{ML}\end{array}$ & DJ\#3 Pg. 117 \\
\hline 2009_10_18 & $\begin{array}{l}\text { Ag deposition on } \mathrm{NiAl}(110) \text { at } 185 \mathrm{~K} \\
\text { Flux: } 1 \times 10^{-2} \mathrm{ML} / \mathrm{s} \\
\text { Coverage: } 0.3 \mathrm{ML}\end{array}$ & DJ\#3 Pg. 123 \\
\hline
\end{tabular}

Table 1 Continued 


\begin{tabular}{|l|l|l|}
\hline Folder name & Experiment description & Notes \\
\hline $2009 \_10 \_21$ & Failed Au deposition on NiAl(110) experiment & DJ\#3 Pg. 125 \\
\hline $2009 \_10 \_23$ & $\begin{array}{l}\text { Ag deposition on NiAl(110) at } 225 \mathrm{~K} \\
\text { Flux: } 1 \times 10^{-2} \mathrm{ML} / \mathrm{s} \\
\text { Coverage: } 0.3 \mathrm{ML}\end{array}$ & DJ\#3 Pg. 127 \\
\hline $2009 \_10 \_28$ & Failed Au deposition on NiAl(110) experiment & DJ\#3 Pg. 130 \\
\hline $2009 \_10 \_30$ & $\begin{array}{l}\text { Ag deposition on NiAl(110) at } 205 \mathrm{~K} \\
\text { Flux: } 1 \times 10^{-2} \mathrm{ML} / \mathrm{s} \\
\text { Coverage: } 0.3 \mathrm{ML}\end{array}$ & DJ\#3 Pg. 131 \\
\hline
\end{tabular}

Table 1

a: The folders appear in DJ's data under Raw data/STM.

b: The notes represent the page and the experiment notebook. (e.g. FQ\#2 Pg. 66 means page 66 of Dr. Feili Qin's notebook \#2) 
Table 2

\begin{tabular}{|c|c|c|}
\hline Folder name & Experiment description & Notes \\
\hline 2007_10_20 & $\begin{array}{l}\text { NiAl(110) clean surface LEED } \\
3 \mathrm{ML} \mathrm{Ag} / \mathrm{NiAl}(110) \mathrm{LEED} \\
10 \mathrm{ML} \mathrm{Ag} / \mathrm{NiAl}(110) \mathrm{LEED} \\
\text { Flux: } 6.7 \times 10^{-3} \mathrm{ML} / \mathrm{s}\end{array}$ & BU\#3 Pg. 18 \\
\hline 2010_01_23 & NiAl(110) clean surface LEED: $70-300 \mathrm{~V}$ & DJ\#3 Pg. 146 \\
\hline 2010_01_24 & $\begin{array}{l}\text { NiAl(110) clean surface LEED: } 50-300 \mathrm{~V} \\
\text { Several Ag deposition trials, not successful }\end{array}$ & DJ\#3 Pg. 147 \\
\hline 2010_01_25 & $\begin{array}{l}\text { NiAl(110) clean surface LEED: } 80-300 \mathrm{~V} \\
\text { Ag deposition trial, not successful }\end{array}$ & DJ\#3 Pg. 150 \\
\hline 2010_02_07 & $\begin{array}{l}\text { NiAl(110) clean surface LEED: } 75-300 \mathrm{~V} \\
\text { Several Ag deposition trial, not successful }\end{array}$ & DJ\#4 Pg. 4 \\
\hline 2010_02_09 & $\begin{array}{l}\text { NiAl(110) clean surface LEED: } 75-270 \mathrm{~V} \\
\text { Ag deposition trial, not successful }\end{array}$ & DJ\#4 Pg. 8 \\
\hline 2010_02_27 & NiAl(110) clean surface LEED: $95-270 \mathrm{~V}$ & DJ\#4 Pg. 15 \\
\hline 2010_04_08 & $\begin{array}{l}\text { NiAl(110) clean surface LEED: } 120-300 \mathrm{~V} \\
6 \mathrm{ML} \mathrm{Ag} / \mathrm{NiAl}(110) \text { LEED: } 145-300 \mathrm{~V}\end{array}$ & DJ\#4 Pg. 38 \\
\hline 2010_04_09 & $\begin{array}{l}6 \mathrm{ML} \text { Ag/NiAl(110) LEED: } 190-300 \mathrm{~V} \\
24 \mathrm{ML} \mathrm{Ag} / \mathrm{NiAl}(110) \text { LEED: } 160-300 \mathrm{~V}\end{array}$ & DJ\#4 Pg. 44 \\
\hline 2010_04_11 & $48 \mathrm{ML}$ Ag/NiAl(110) LEED: $175-300 \mathrm{~V}$ & DJ\#4 Pg. 49 \\
\hline 2010_04_13 & $3 \mathrm{ML}$ Ag/NiAl(110) LEED: $170-300 \mathrm{~V}$ & DJ\#4 Pg. 49 \\
\hline 2010_04_23 & $\begin{array}{l}\text { NiAl(110) clean surface LEED: } 185-300 \mathrm{~V} \\
1 \mathrm{ML} \mathrm{Ag} / \mathrm{NiAl}(110) \text { LEED: } 185-300 \mathrm{~V} \\
2 \mathrm{ML} \mathrm{Ag} / \mathrm{NiAl}(110) \text { LEED: } 185-300 \mathrm{~V}\end{array}$ & DJ\#4 Pg. 53 \\
\hline 2010_04_27 & $\begin{array}{l}\text { NiAl(110) clean surface LEED: } 185-330 \mathrm{~V} \\
9 \mathrm{ML} \text { Ag/NiAl(110) LEED: } 210-300 \mathrm{~V} \\
12 \mathrm{ML} \text { Ag/NiAl(110) LEED: } 200-300 \mathrm{~V}\end{array}$ & DJ\#4 Pg. 56 \\
\hline 2010_05_15 & $\begin{array}{l}24 \mathrm{ML} \text { Ag/NiAl(110) LEED: } 195-300 \mathrm{~V} \\
48 \mathrm{ML} \mathrm{Ag} / \mathrm{NiAl}(110) \text { LEED: } 190-300 \mathrm{~V} \\
96 \mathrm{ML} \mathrm{Ag} / \mathrm{NiAl}(110) \text { LEED: } 185-300 \mathrm{~V} \\
192 \mathrm{ML} \mathrm{Ag} / \mathrm{NiAl}(110) \text { LEED: } 175-300 \mathrm{~V}\end{array}$ & DJ\#4 Pg. 60 \\
\hline 2010_05_16 & $\begin{array}{l}384 \mathrm{ML} \text { Ag/NiAl(110) LEED: } 220-300 \mathrm{~V} \\
768 \mathrm{ML} \mathrm{Ag} / \mathrm{NiAl}(110) \text { LEED: } 210-300 \mathrm{~V}\end{array}$ & DJ\#4 Pg. 65 \\
\hline
\end{tabular}

Table 2 


\section{ACKNOWLEGEMENTS}

I am heartily thankful to my advisor Professor Patricia A. Thiel for her guidance, support and encouragement throughout my graduate study and my life in Ames. During the five years of research in Thiel group, Pat has leaded me to the field of surface science and her enthusiasm in research has inspired me so deeply in my own research projects. I greatly appreciate her sound advice, good teaching and many good ideas she provided me throughout my thesis-writing period. Without her, I would have been lost.

I would like to express my sincere gratitude to Professor James W. Evans for his support and guidance. I have always enjoyed discussing research with him. His way of explaining things so clearly and simply always makes me feel amazed.

I have to thank all Thiel group members, past and present, for providing me a cooperative, friendly and supportive working atmosphere. Dr. Cynthia Jenks taught me the principles of ultrahigh vacuum technology. Dr. Mingmin Shen helped me when I first started doing experiments. I would also like to acknowledge the people I worked close with, including Dr. Thomas Duguet, Chad Yuen, Selena Russell, Alex Belianinov and Dahai Shao.

My further gratitude goes to Dr. Yong Han from Evans group. His work includes DFT calculations and KMC simulations. His beautiful theoretical work has helped me so much in elucidating my experimental observations.

Many thanks to Jim Anderegg for his help with instrument trouble-shooting. He also gave me many valuable tips on how to fix problems.

Last but now least, I wish to thank my family for their support and love. This thesis is dedicated to them to express my great gratitude. 
The work presented in this thesis was performed at Ames Laboratory, which is operated by Iowa State University for US Department of Energy under Contract No. DEAC02-07CH11358. This work was supported by NSF grant CHE-0809472. 